\title{
Discovery of an Oxidative System for Radical Generation from Csp3-H Bonds: A Synthesis of Functionalized \\ Oxindoles
}

Ming-Zhong Zhang, ${ }^{\text {a }}$ Wan-Ting Li, ${ }^{\text {a }}$ Yuan-Yuan Li, ${ }^{a}$ Qi Wang, ${ }^{\text {a }}$ Chong Li, ${ }^{\text {a }}$ Yan-Hao Liu, ${ }^{\text {a }}$ Jin-Xing Yin, ${ }^{\mathrm{a}}$ Xin Yang, ${ }^{\mathrm{a}}$ Huisheng Huang, ${ }^{\mathrm{a}}$ and Tieqiao Chen*b

${ }^{a}$ Chongqing Key Laboratory of Inorganic Special Functional Materials, College of Chemistry and Chemical Engineering, Yangtze Normal University, Chongqing 408100, China.

*E-mail: mzzhang@yznu.edu.cn

${ }^{b}$ Key Laboratory of Ministry of Education for Advanced Materials in Tropical Island Resources, Hainan Provincial Key Lab of Fine Chem, Hainan University, Haikou, 570228, China.

*E-mail: chentieqiao@hnu.edu.cn

Table of Contents

${ }^{1} \mathrm{H},{ }^{13} \mathrm{C}\left\{{ }^{1} \mathrm{H}\right\}$ and ${ }^{19} \mathrm{~F}$ NMR spectra

S2-S72 
${ }^{1} \mathrm{H},{ }^{13} \mathrm{C}\left\{{ }^{1} \mathrm{H}\right\}$ and ${ }^{19} \mathrm{~F}$ NMR spectra

${ }^{1} \mathrm{H}$ NMR of 3aa-1 (400 MHz, $\left.\mathrm{CDCl}_{3}\right)$

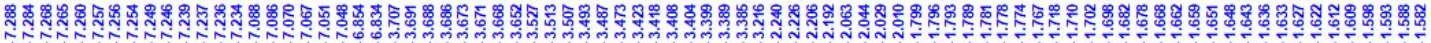

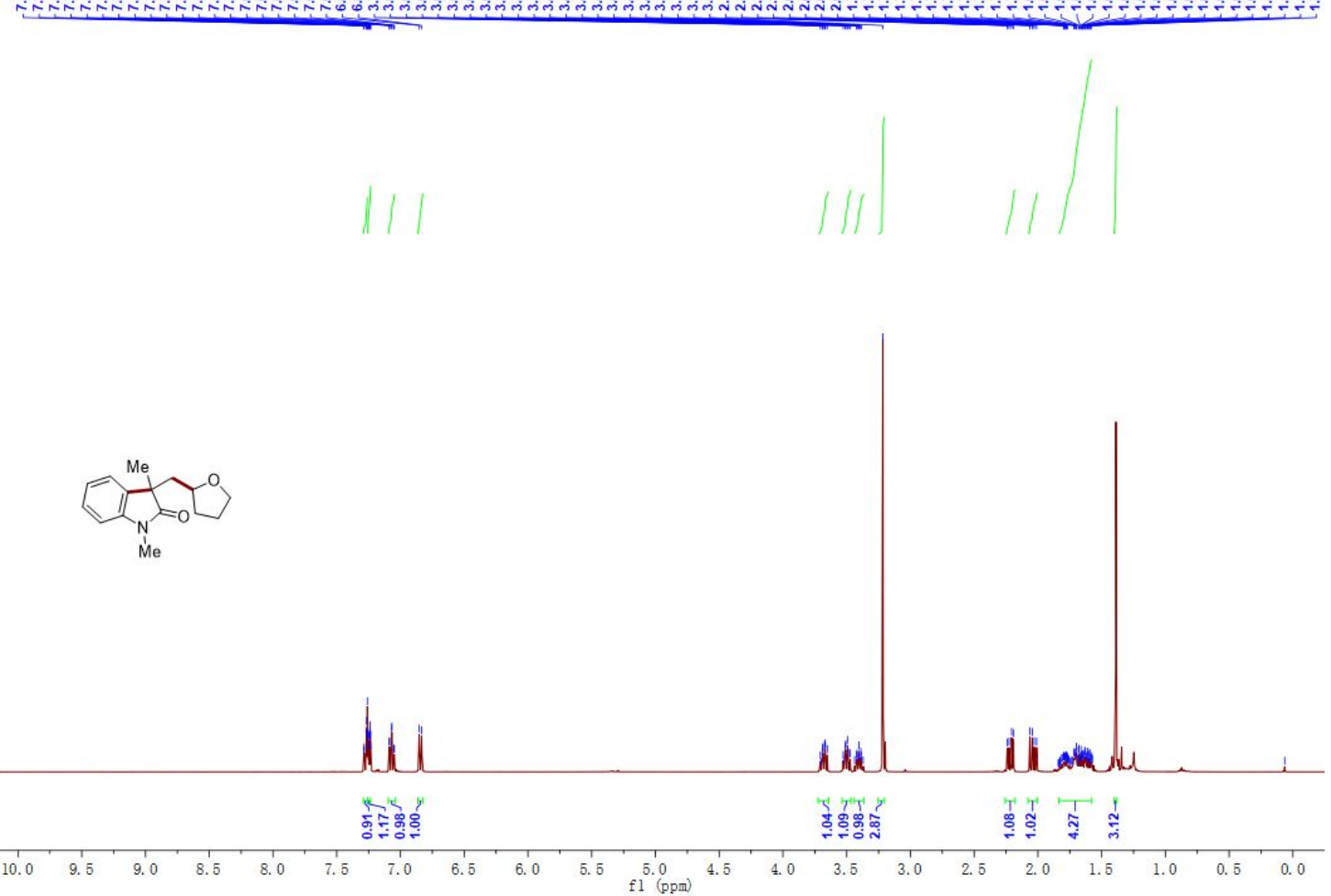

${ }^{13} \mathrm{C}\left\{{ }^{1} \mathrm{H}\right\}$ NMR of 3aa-1 (100 MHz, $\left.\mathrm{CDCl}_{3}\right)$

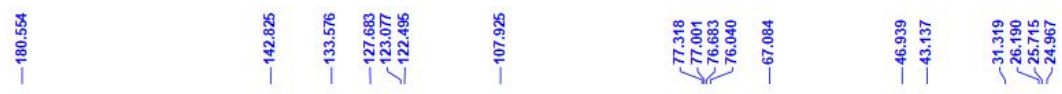

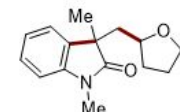

Me
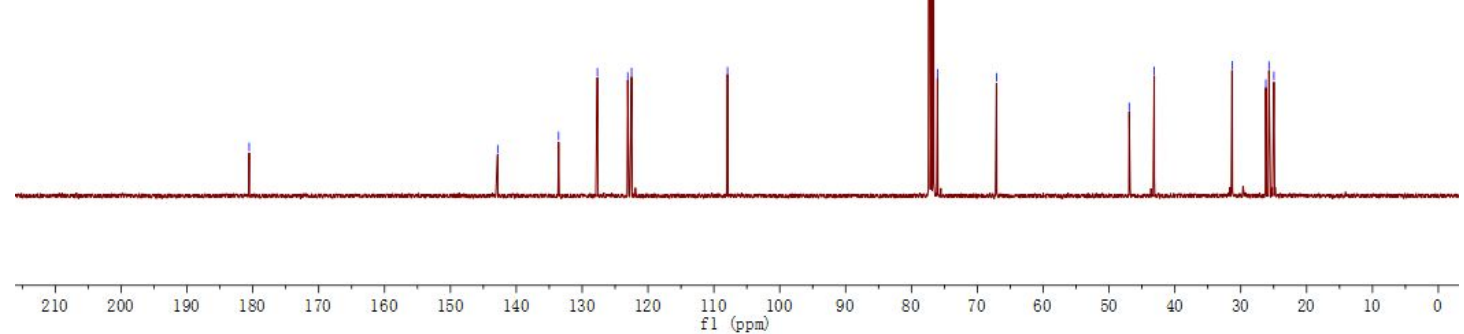
${ }^{1} \mathrm{H}$ NMR of 3aa-2 (400 MHz, $\left.\mathrm{CDCl}_{3}\right)$

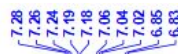

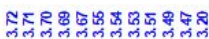

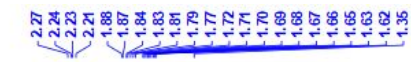

Me
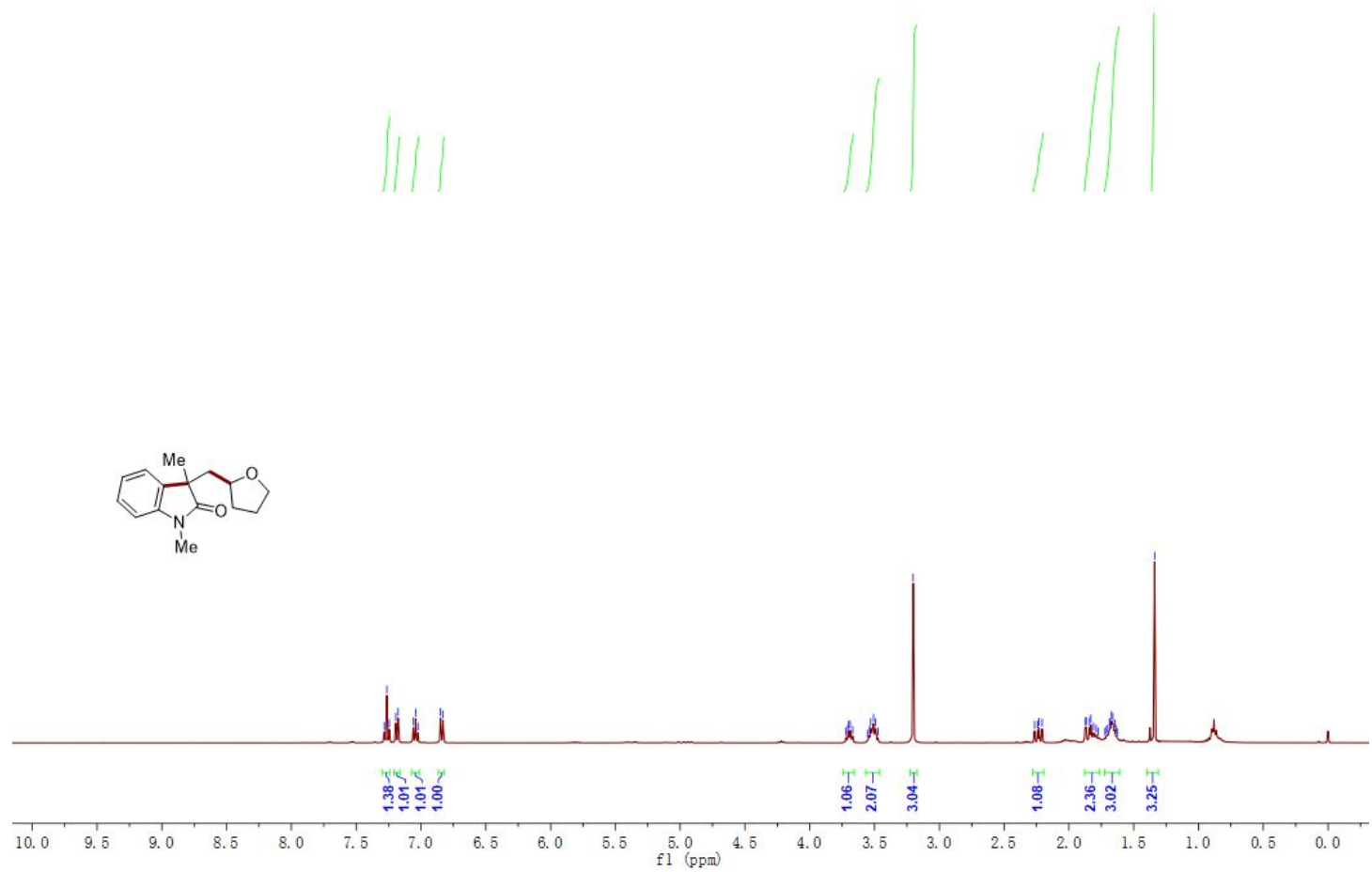

${ }^{13} \mathrm{C}\left\{{ }^{1} \mathrm{H}\right\}$ NMR of 3aa-2 $\left(100 \mathrm{MHz}, \mathrm{CDCl}_{3}\right)$

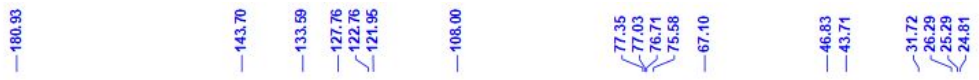

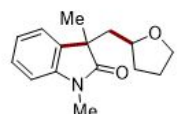

Me

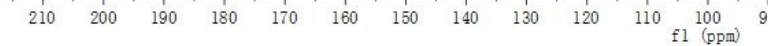


${ }^{1} \mathrm{H}$ NMR of 3ab-1 (500 MHz, $\left.\mathrm{CDCl}_{3}\right)$

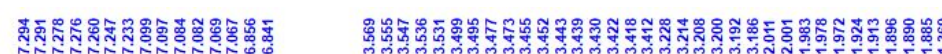
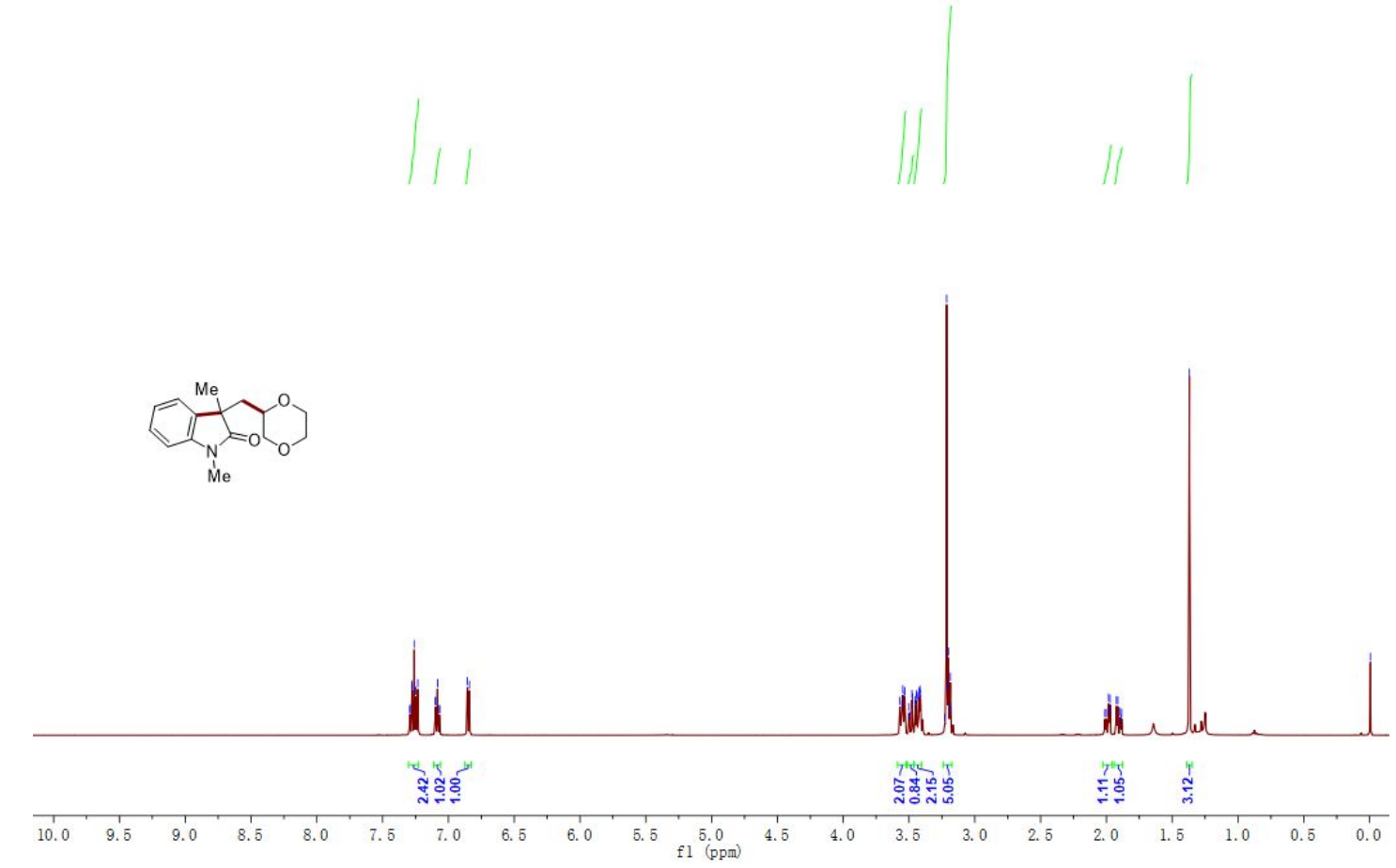

${ }^{13} \mathrm{C}\left\{{ }^{1} \mathrm{H}\right\}$ NMR of 3ab-1 (125 MHz, $\left.\mathrm{CDCl}_{3}\right)$

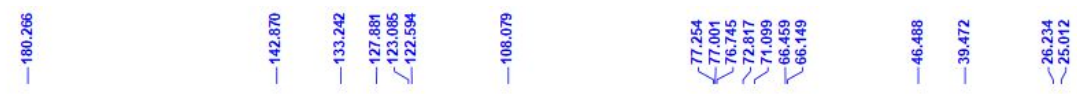

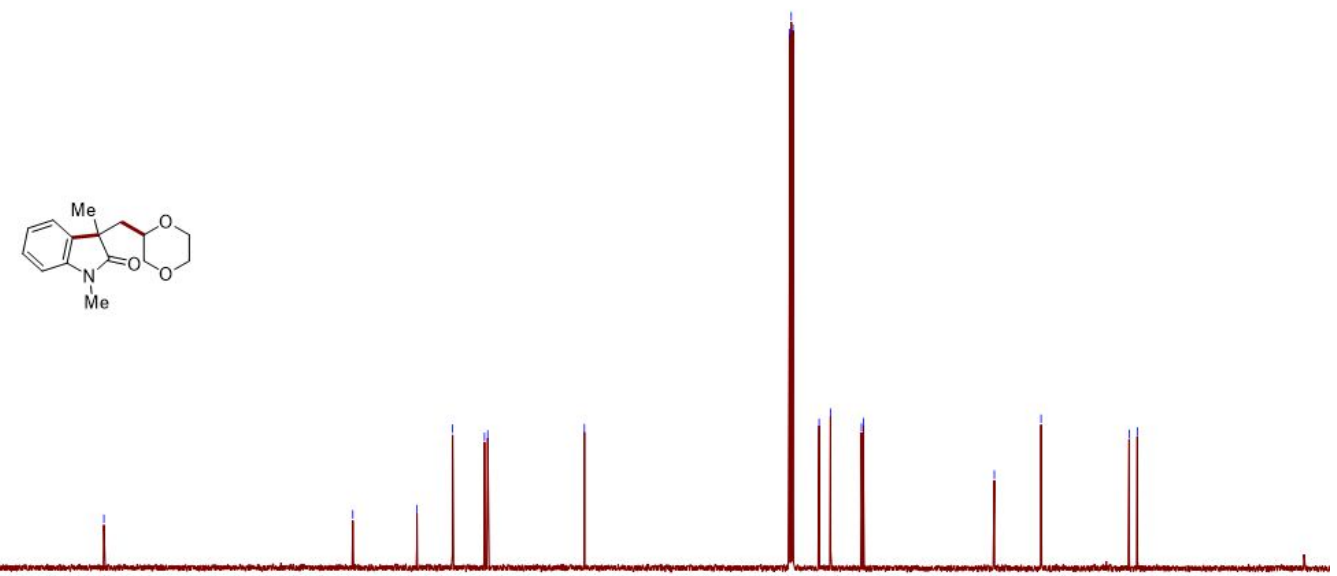

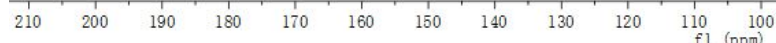

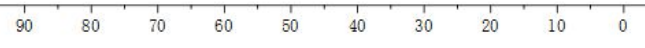


${ }^{1} \mathrm{H}$ NMR of 3ab-2 $\left(500 \mathrm{MHz}, \mathrm{CDCl}_{3}\right)$

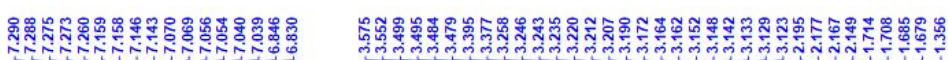
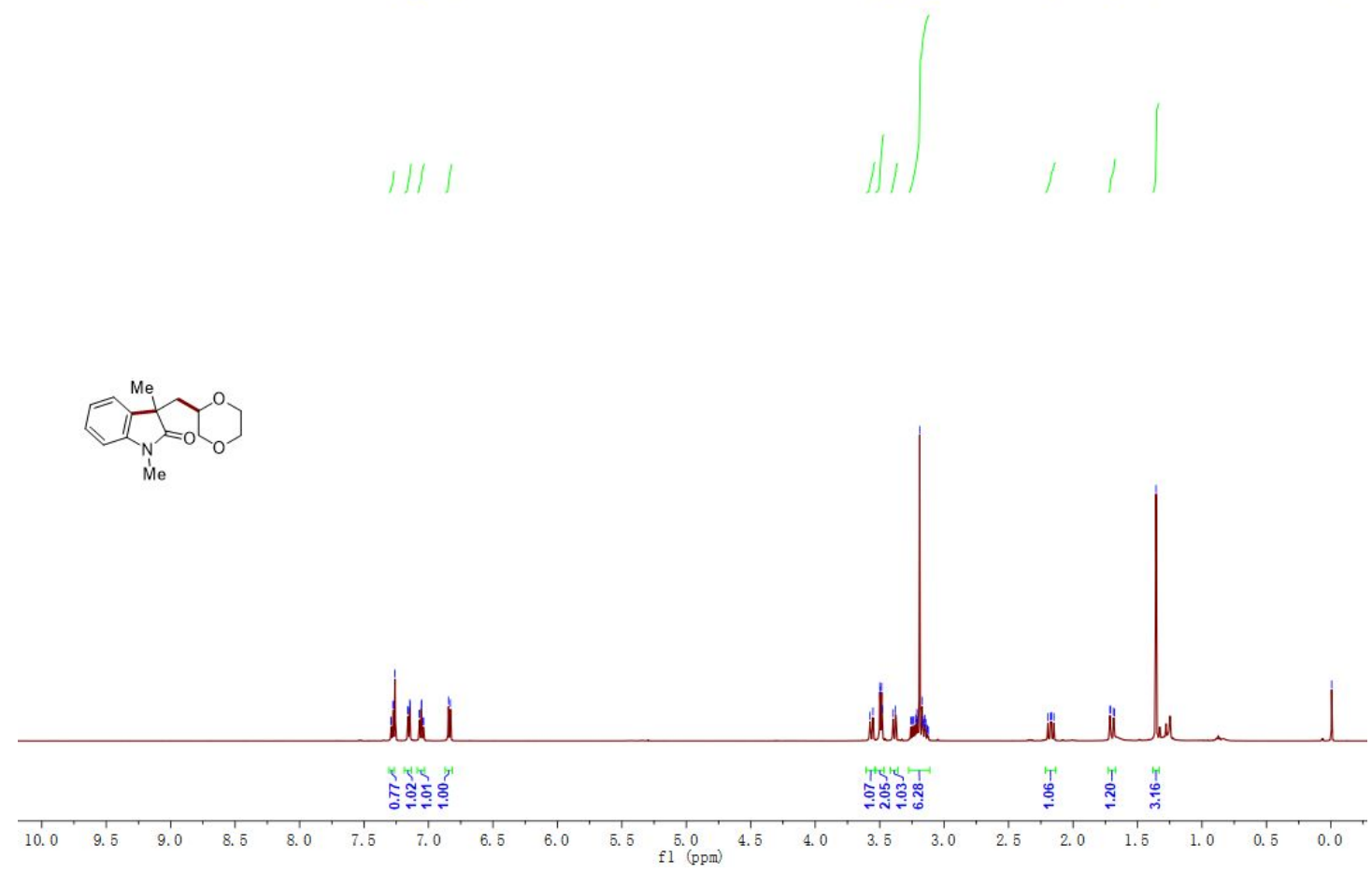

${ }^{13} \mathrm{C}\left\{{ }^{1} \mathrm{H}\right\}$ NMR of $\mathbf{3 a b}-2\left(125 \mathrm{MHz}, \mathrm{CDCl}_{3}\right)$

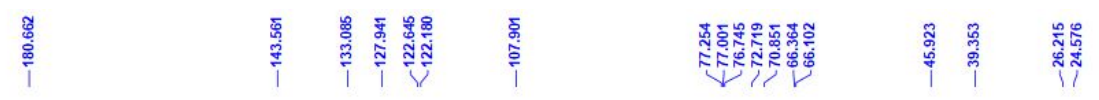

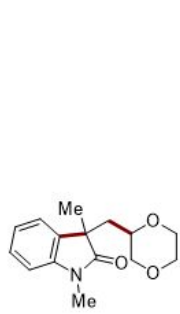

Me

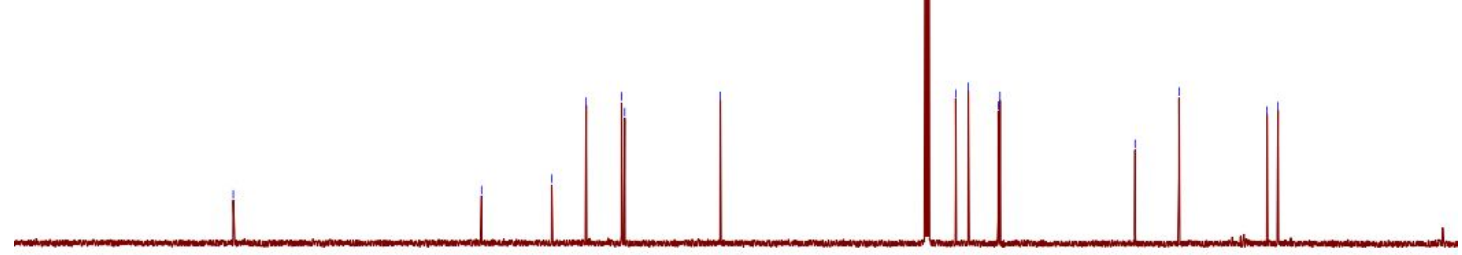

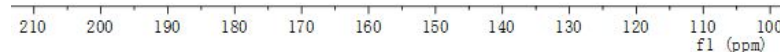


${ }^{1} \mathrm{H}$ NMR of $\mathbf{3 b b}-\mathbf{1}\left(500 \mathrm{MHz}, \mathrm{CDCl}_{3}\right)$

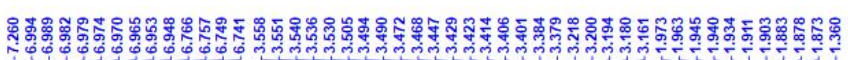

홍

$\underbrace{M e}_{\substack{n \\ n}=0}$
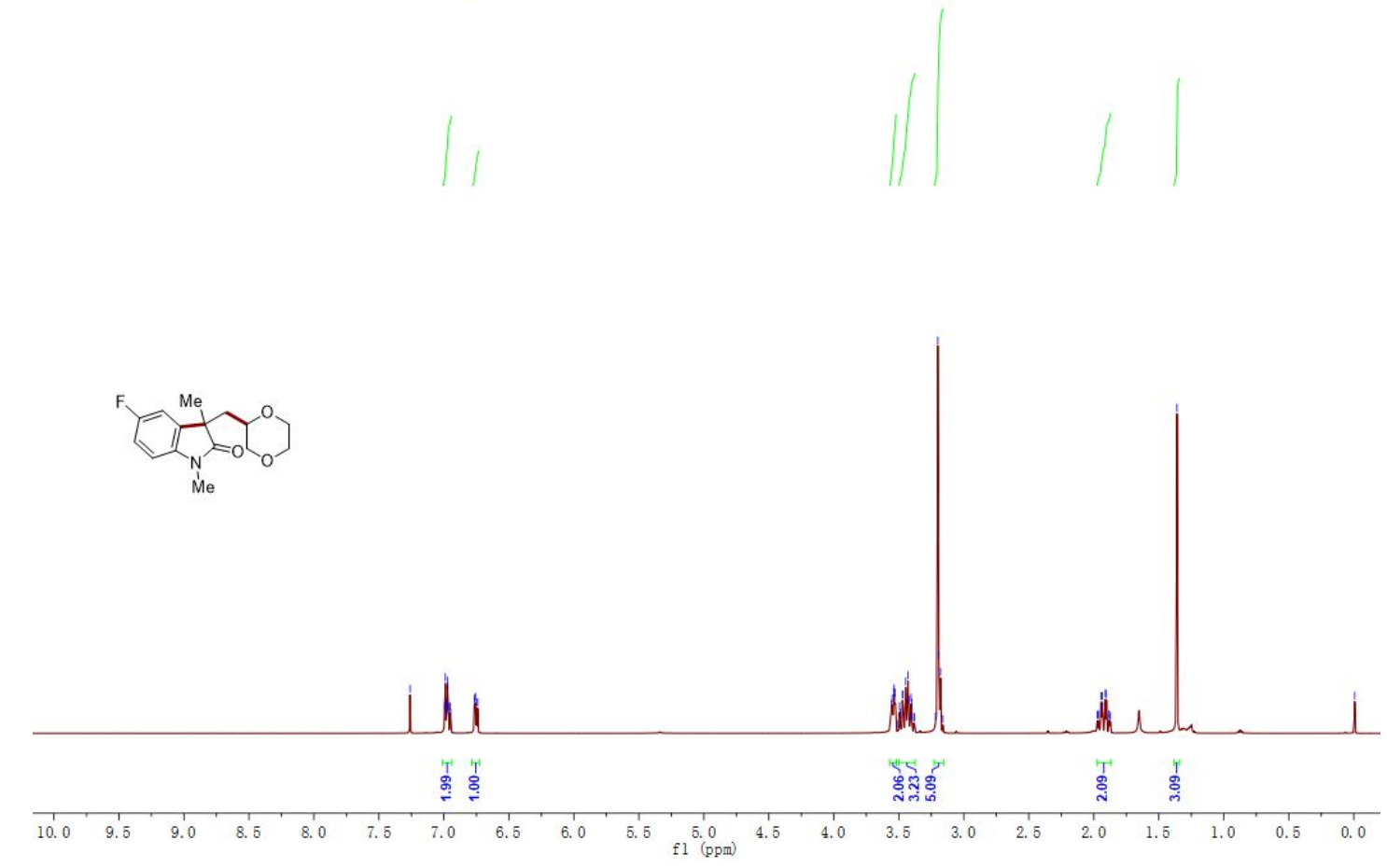

${ }^{13} \mathrm{C}\left\{{ }^{1} \mathrm{H}\right\}$ NMR of $\mathbf{3 b b}-\mathbf{1}\left(125 \mathrm{MHz}, \mathrm{CDCl}_{3}\right)$

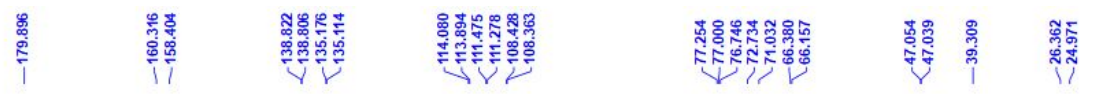

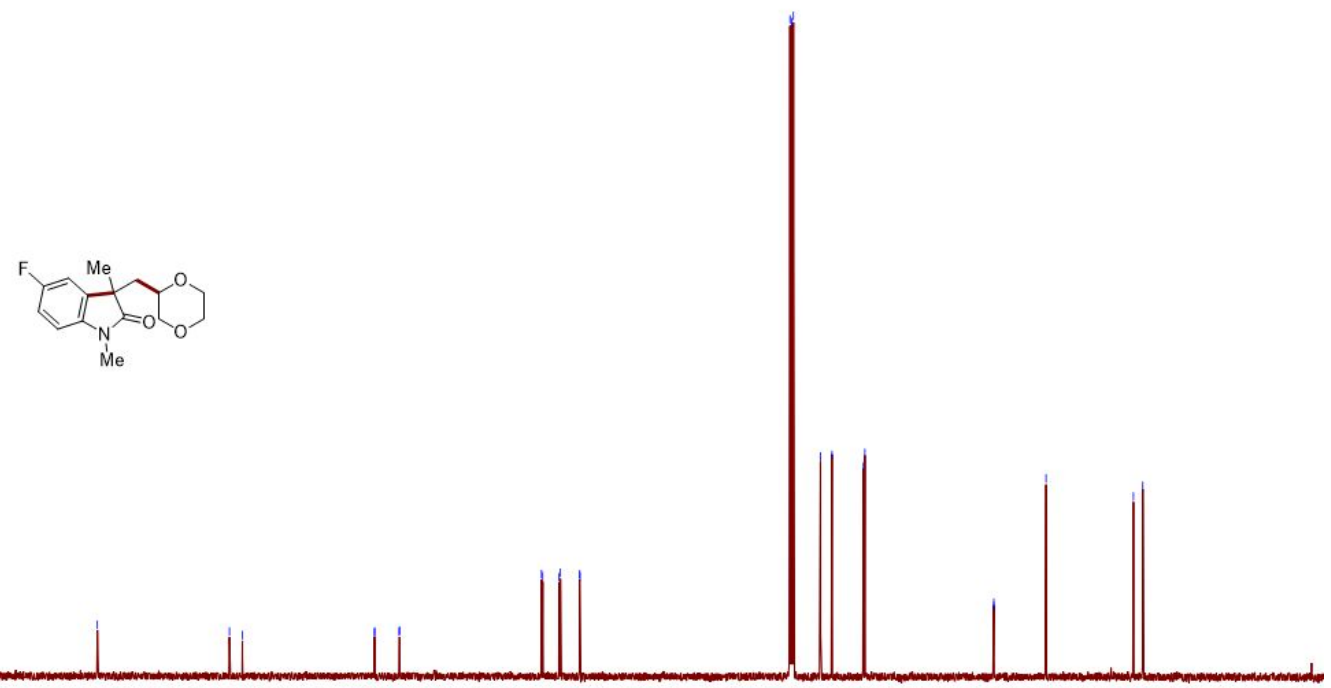

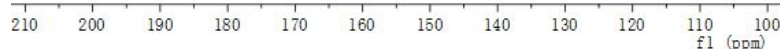


${ }^{19} \mathrm{~F}$ NMR of $\mathbf{3 b b}-\mathbf{1}\left(565 \mathrm{MHz}, \mathrm{CDCl}_{3}\right)$

i

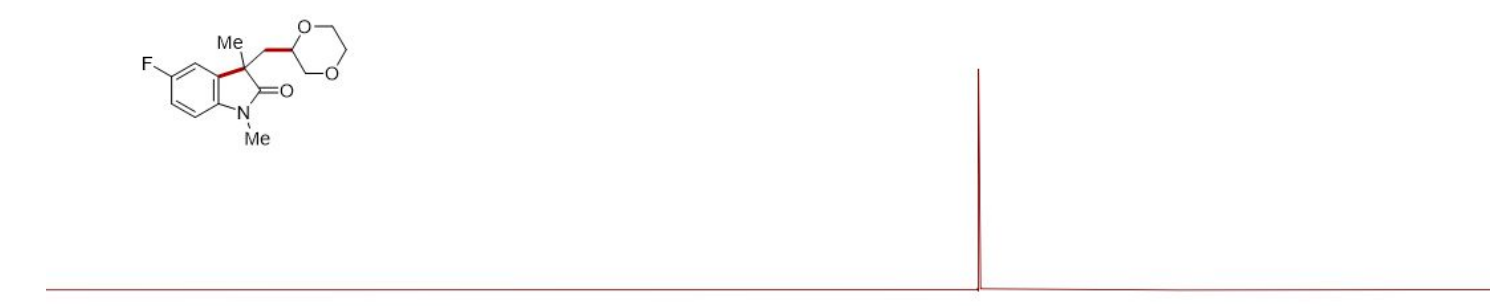

(n)

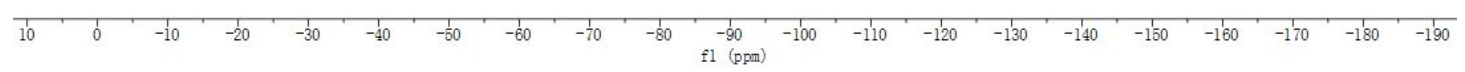

${ }^{1} \mathrm{H}$ NMR of 3bb-2 $\left(500 \mathrm{MHz}, \mathrm{CDCl}_{3}\right)$

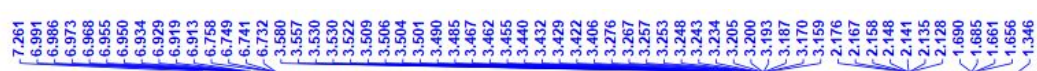
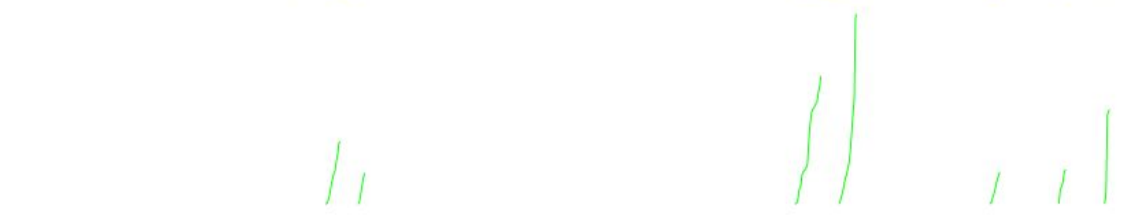

(N)

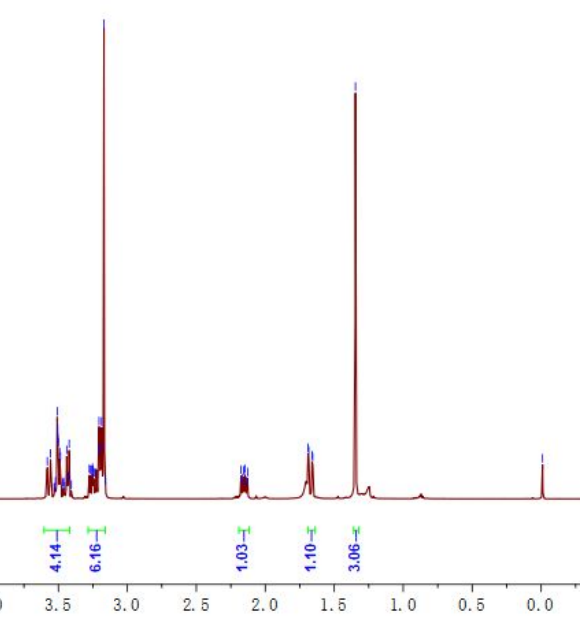


${ }^{13} \mathrm{C}\left\{{ }^{1} \mathrm{H}\right\}$ NMR of $\mathbf{3 b b}-2\left(125 \mathrm{MHz}, \mathrm{CDCl}_{3}\right)$

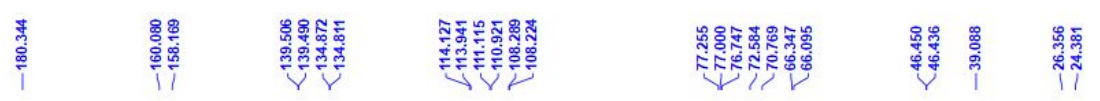
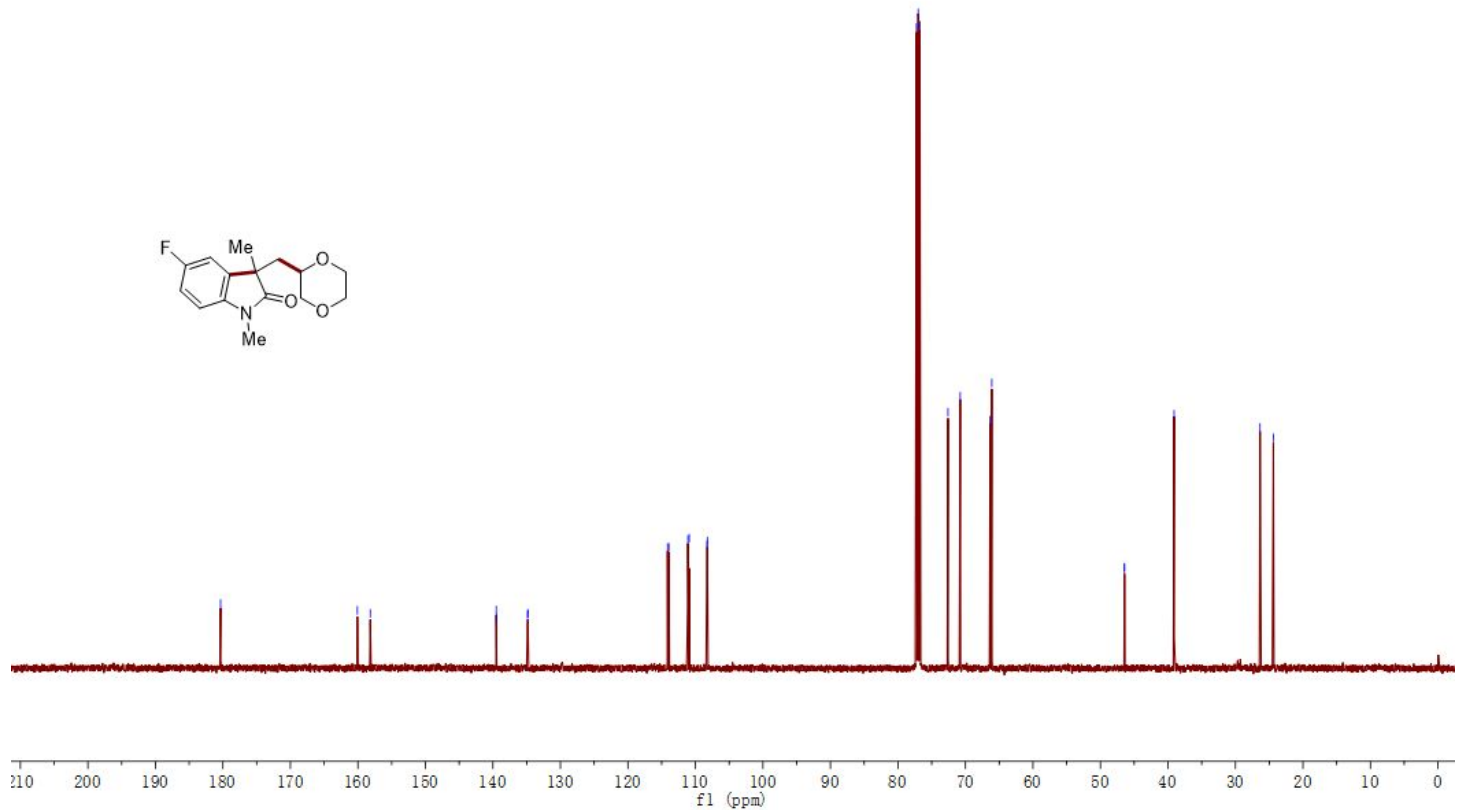

${ }^{19} \mathrm{~F}$ NMR of 3bb-2 $\left(565 \mathrm{MHz}, \mathrm{CDCl}_{3}\right)$

$\underset{i}{i}$

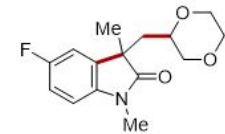

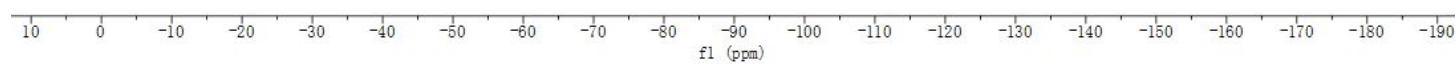


${ }^{1} \mathrm{H}$ NMR of 3cb-1 (500 MHz, $\left.\mathrm{CDCl}_{3}\right)$

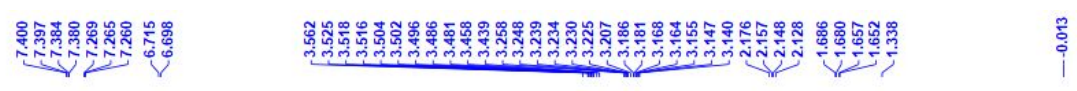

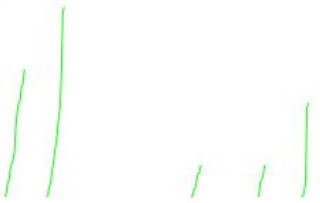

$\underbrace{\mathrm{Me}}_{\substack{n \\ n}=0}=\mathrm{O}_{0}^{\mathrm{O}}$

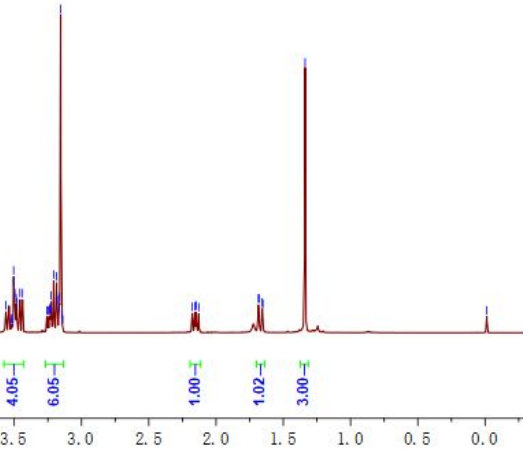

${ }^{13} \mathrm{C}\left\{{ }^{1} \mathrm{H}\right\}$ NMR of $\mathbf{3 c b}-\mathbf{1}\left(125 \mathrm{MHz}, \mathrm{CDCl}_{3}\right)$

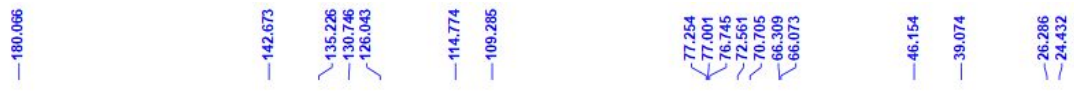

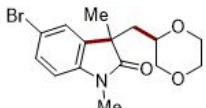

M̀e

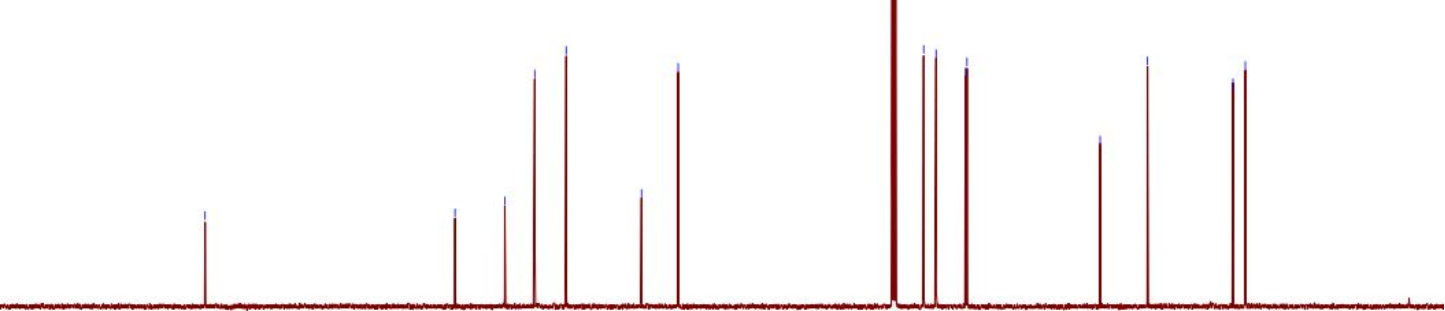

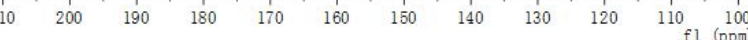

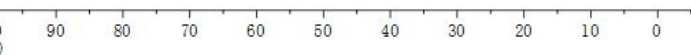


${ }^{1} \mathrm{H}$ NMR of 3db-1 (500 MHz, $\left.\mathrm{CDCl}_{3}\right)$

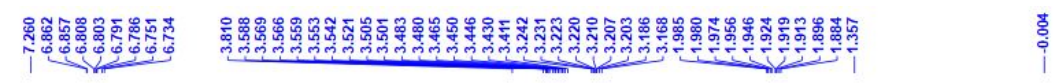

$\mathrm{MeO}_{\substack{\mathrm{Me} \\ \text { MeO }}}^{\mathrm{Me}}$

Me
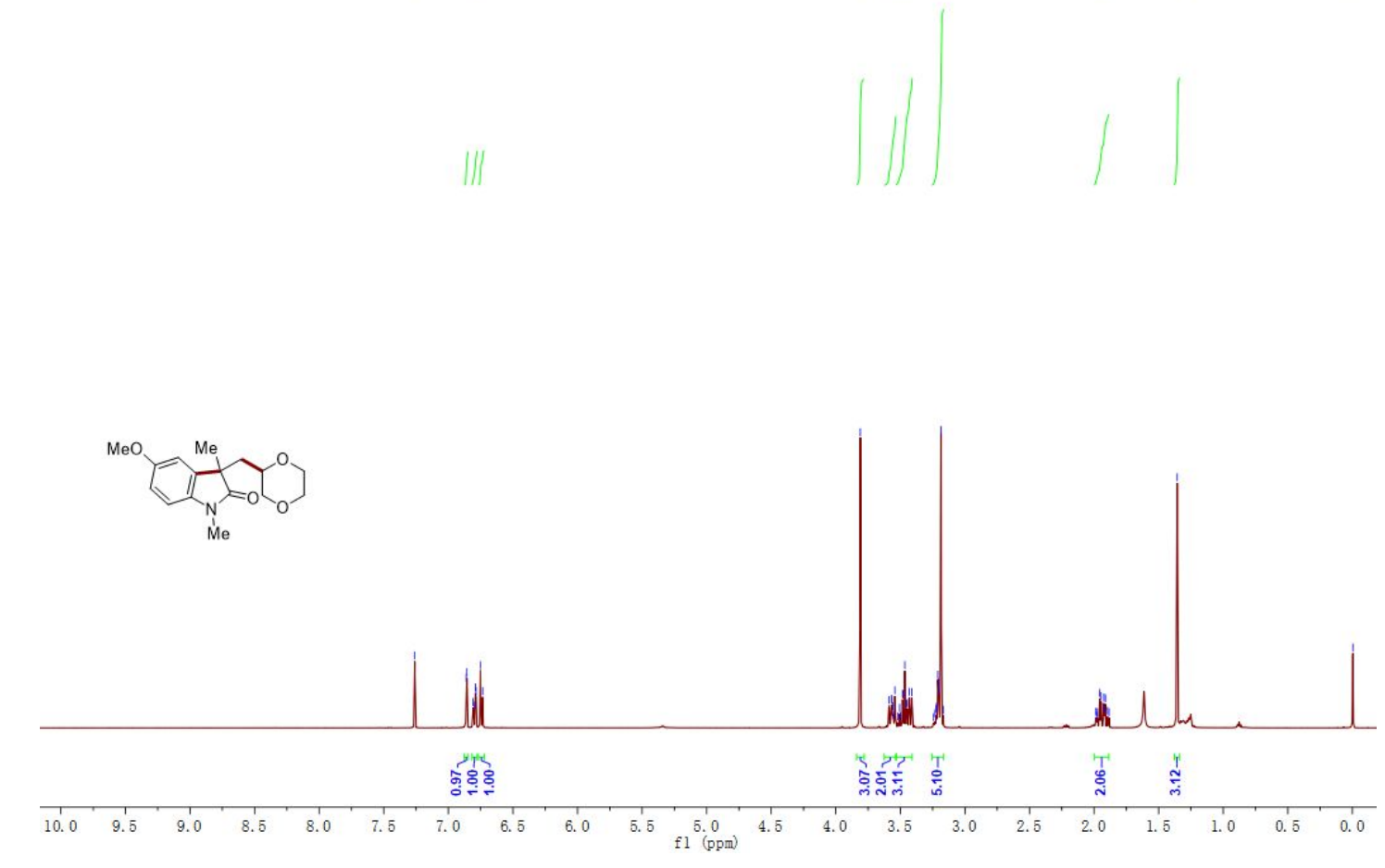

${ }^{13} \mathrm{C}\left\{{ }^{1} \mathrm{H}\right\}$ NMR of 3db-1 (125 MHz, $\left.\mathrm{CDCl}_{3}\right)$

总

旁

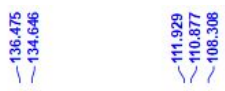

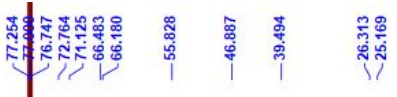

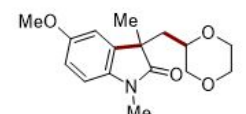

Me

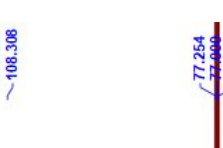

赵赵

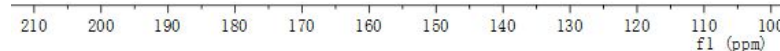


${ }^{1} \mathrm{H}$ NMR of 3db-2 (500 MHz, $\left.\mathrm{CDCl}_{3}\right)$

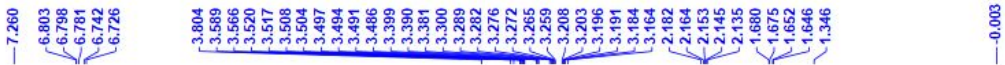

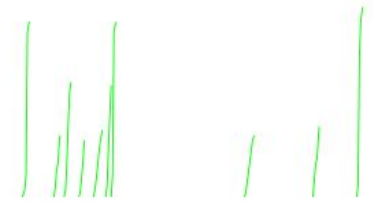

跠

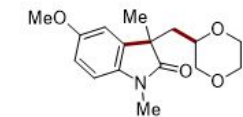
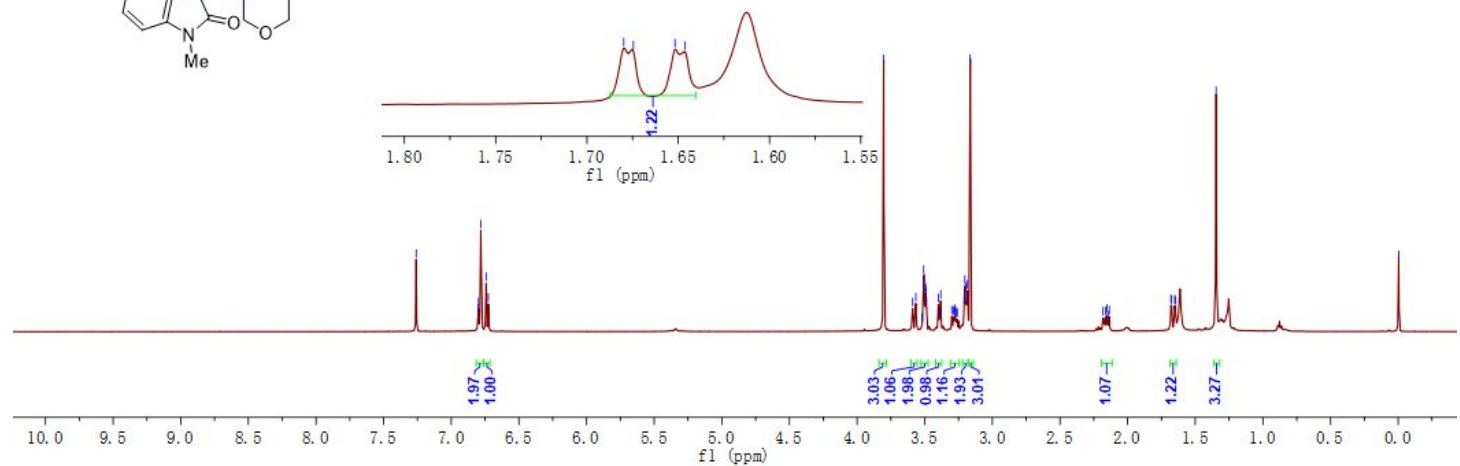

${ }^{13} \mathrm{C}\left\{{ }^{1} \mathrm{H}\right\}$ NMR of 3db-2 (125 MHz, $\left.\mathrm{CDCl}_{3}\right)$

\begin{tabular}{|c|c|c|c|c|c|c|c|}
\hline & 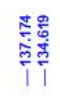 & 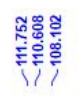 & 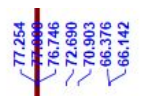 & $\begin{array}{l}\mathscr{8} \\
0 \\
0 \\
0\end{array}$ & 焉 & & 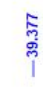 \\
\hline
\end{tabular}

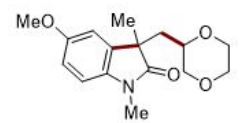

$\mathrm{Me}$

$\begin{array}{llllllllll}190 & 180 & 170 & 160 & 150 & 140 & 130 & 120 & 110 & 100\end{array}$ 
${ }^{1} \mathrm{H}$ NMR of 3eb-1 (500 MHz, $\left.\mathrm{CDCl}_{3}\right)$

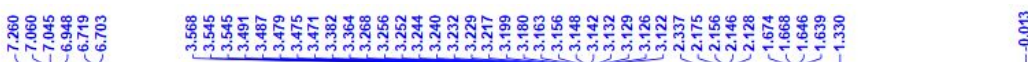
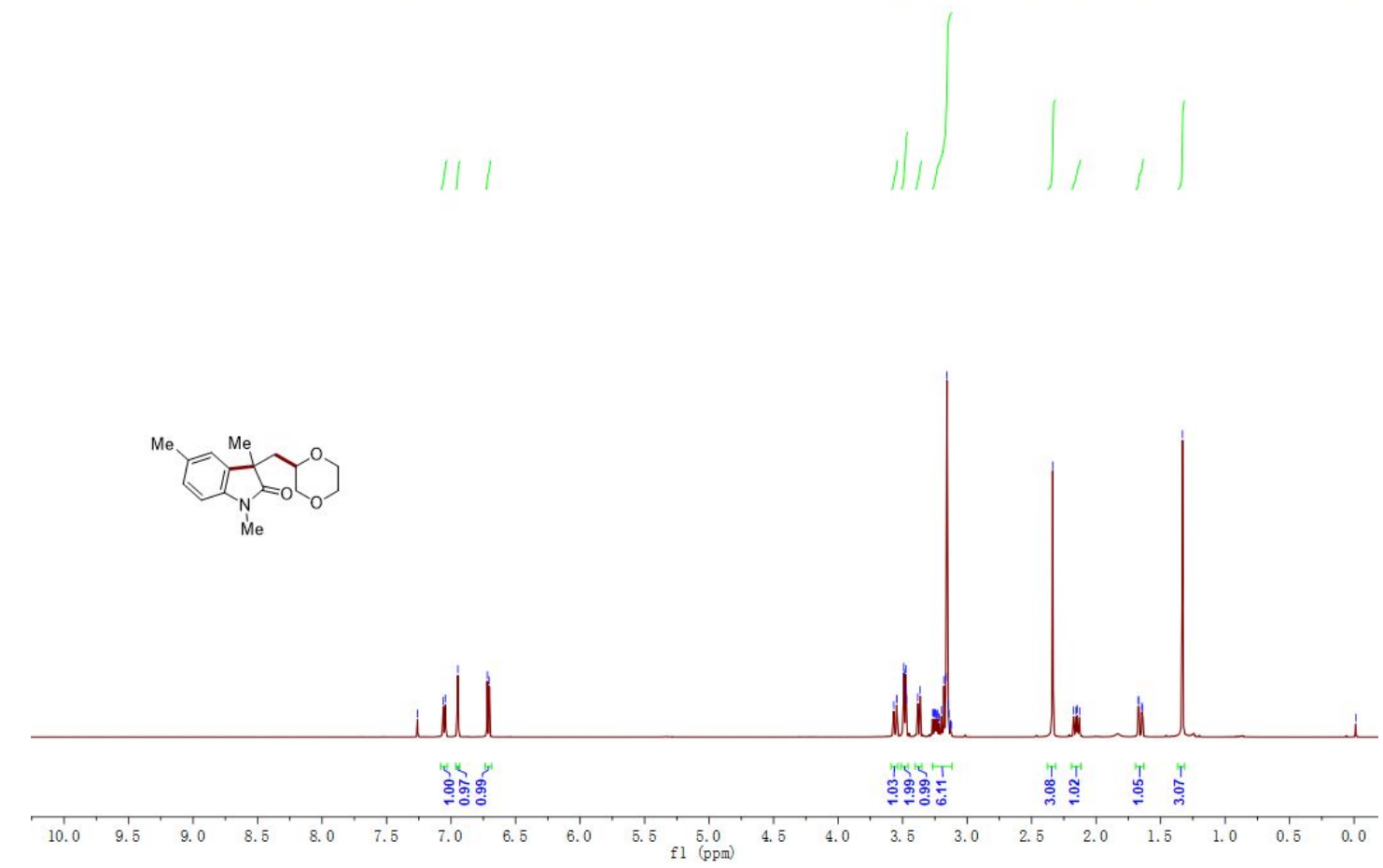

${ }^{13} \mathrm{C}\left\{{ }^{1} \mathrm{H}\right\}$ NMR of 3eb-1 (125 MHz, $\left.\mathrm{CDCl}_{3}\right)$

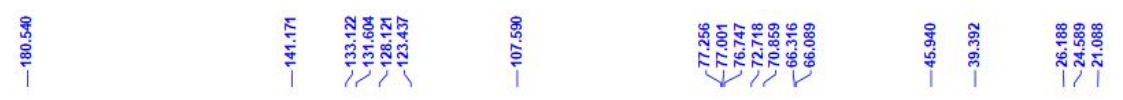

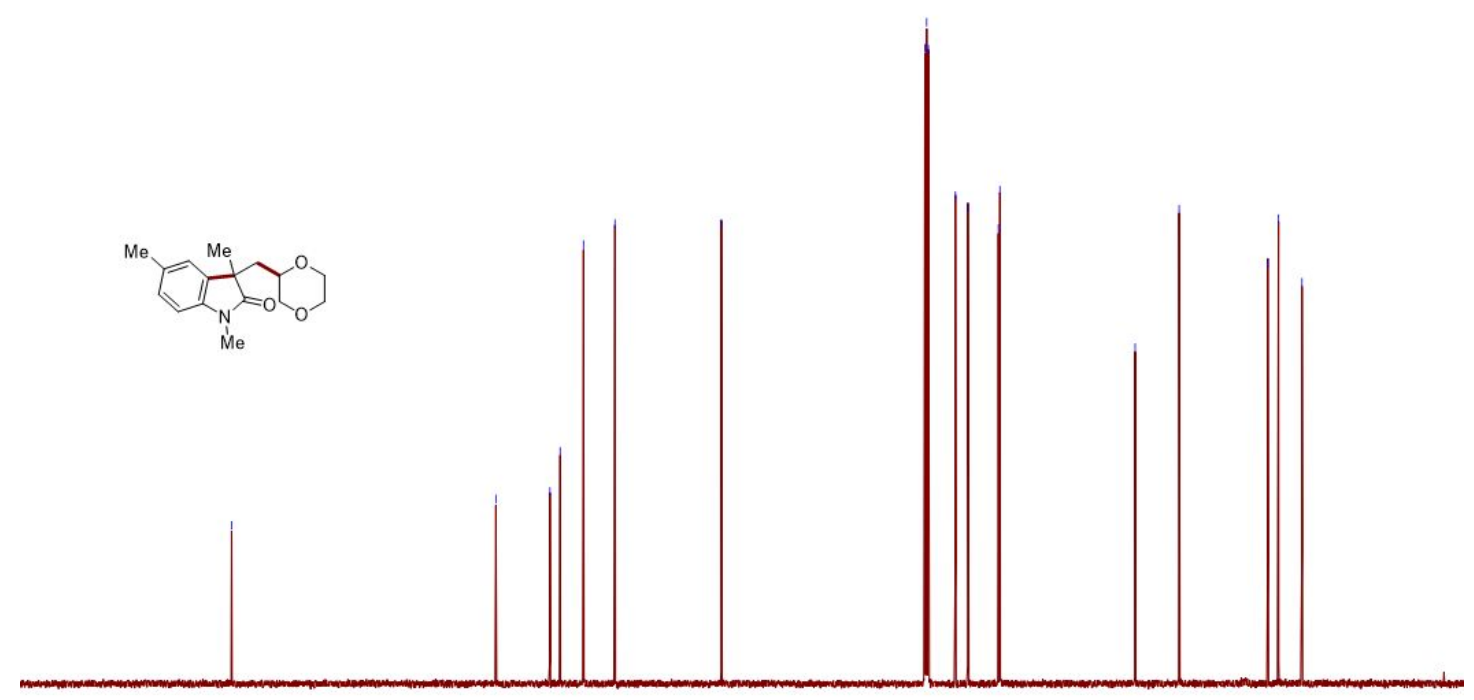

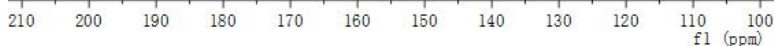


${ }^{1} \mathrm{H}$ NMR of 3eb-2 $\left(500 \mathrm{MHz}, \mathrm{CDCl}_{3}\right)$

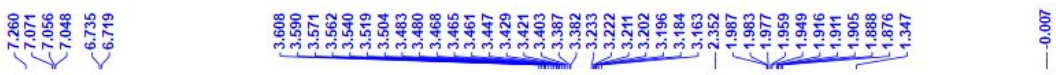

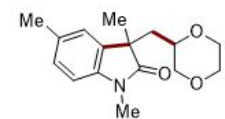
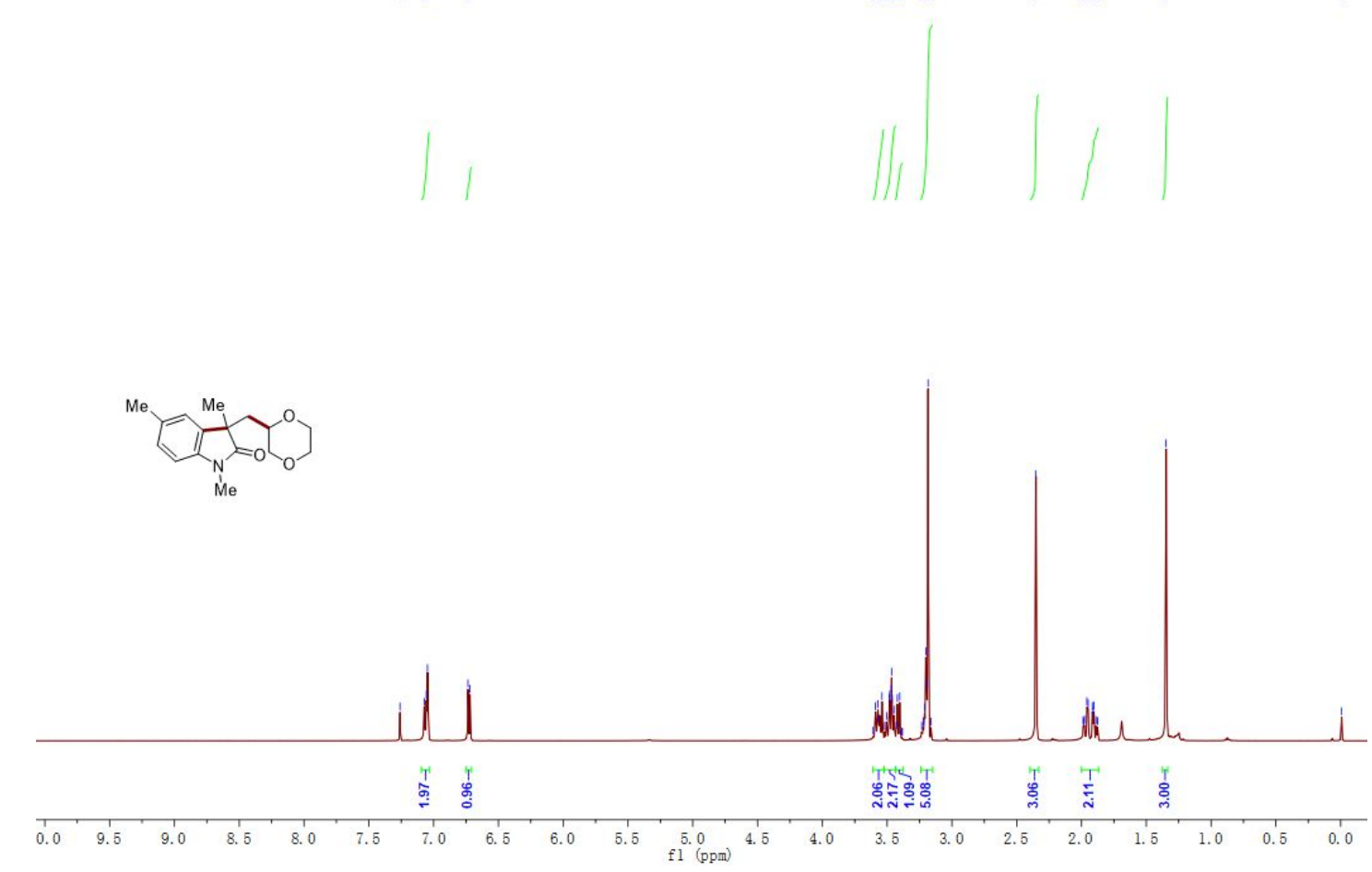

${ }^{13} \mathrm{C}\left\{{ }^{1} \mathrm{H}\right\}$ NMR of $\mathbf{3 e b}-2\left(125 \mathrm{MHz}, \mathrm{CDCl}_{3}\right)$

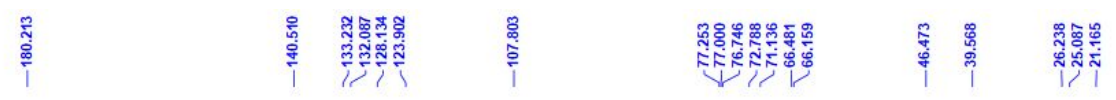
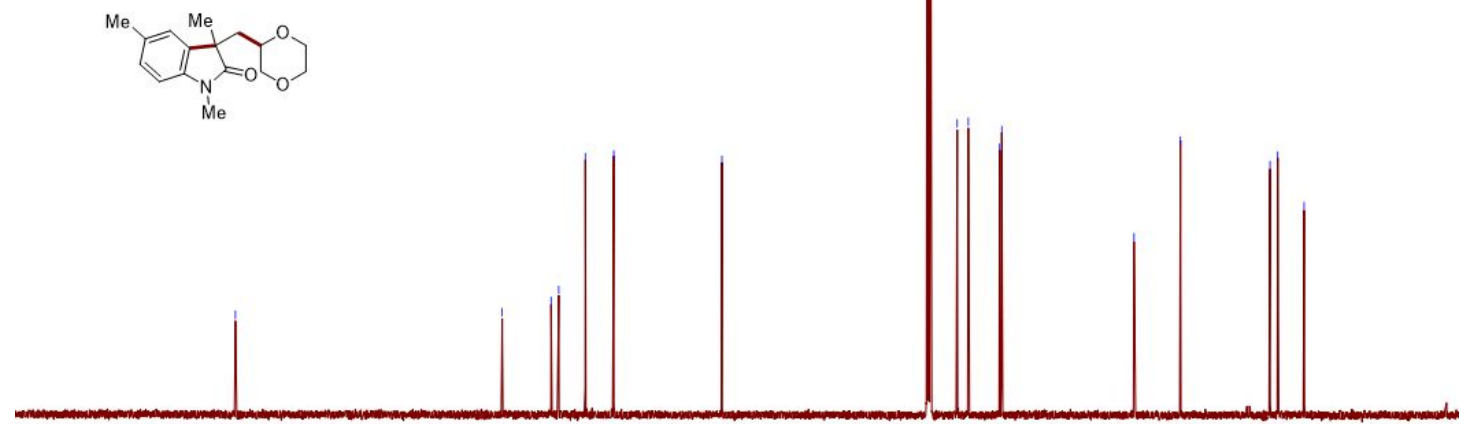

$\begin{array}{llllllllllll}210 & 200 & 190 & 180 & 170 & 160 & 150 & 140 & 130 & 120 & 110 & 100\end{array}$ 
${ }^{1} \mathrm{H}$ NMR of $\mathbf{3 f b}-\mathbf{1}\left(500 \mathrm{MHz}, \mathrm{CDCl}_{3}\right)$

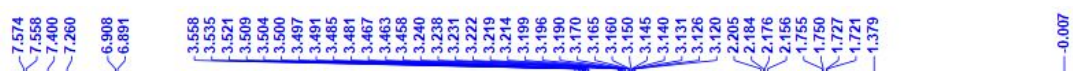

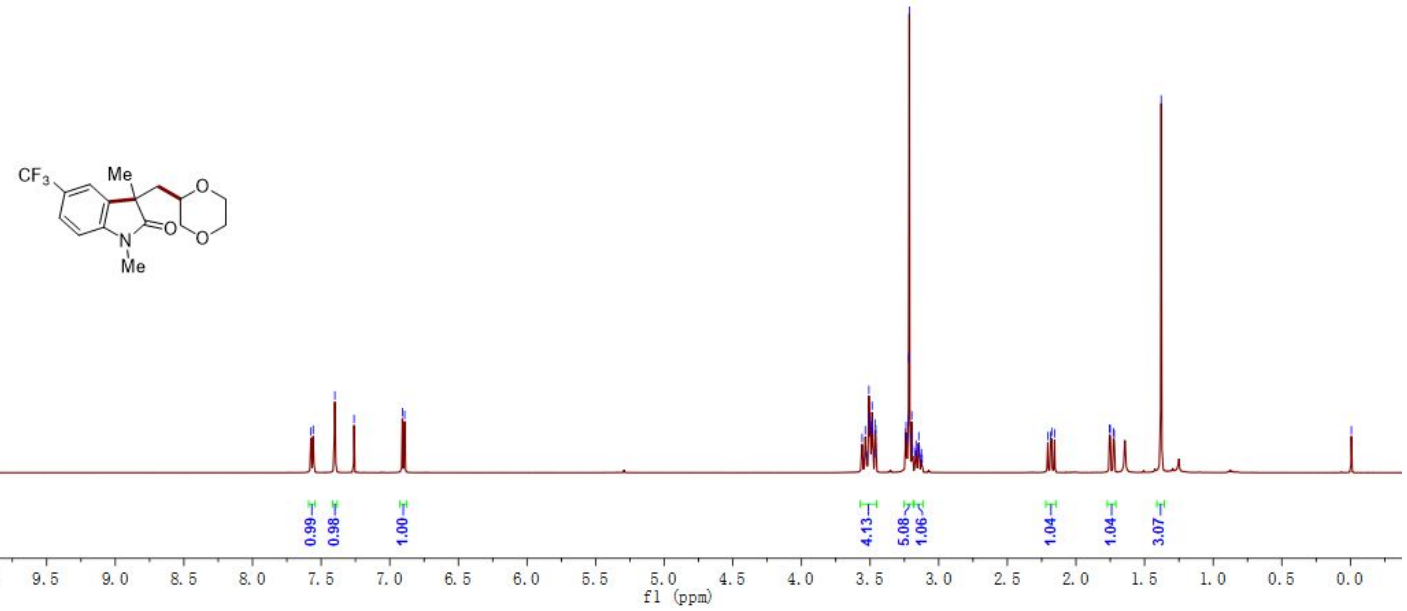

${ }^{13} \mathrm{C}\left\{{ }^{1} \mathrm{H}\right\}$ NMR of $\mathbf{3 f b}-\mathbf{1}\left(125 \mathrm{MHz}, \mathrm{CDCl}_{3}\right)$

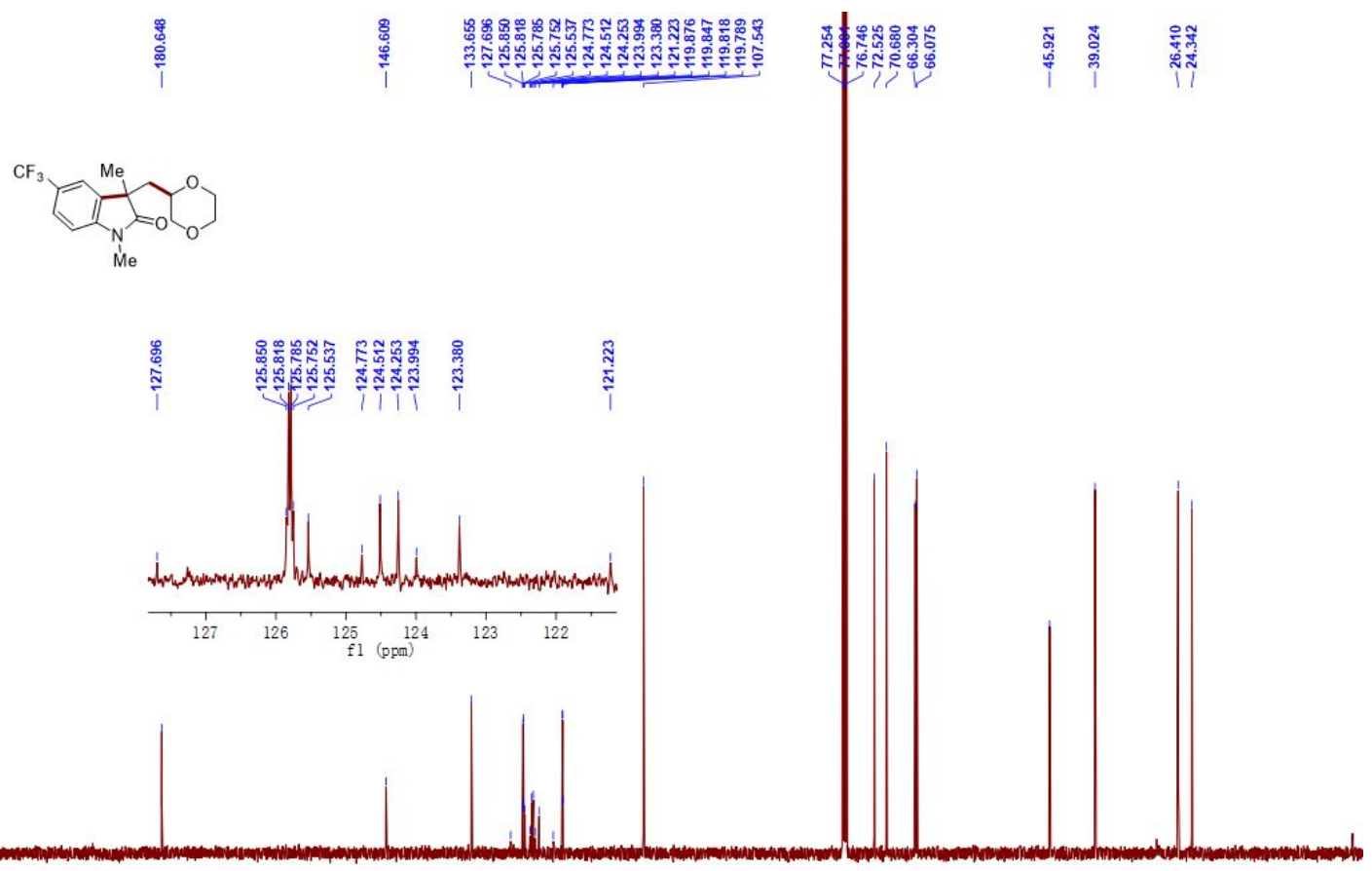

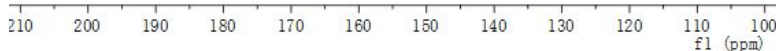


${ }^{19} \mathrm{~F}$ NMR of 3fb-1 (565 MHz, $\left.\mathrm{CDCl}_{3}\right)$

$\stackrel{\infty}{\bar{\varphi}}$
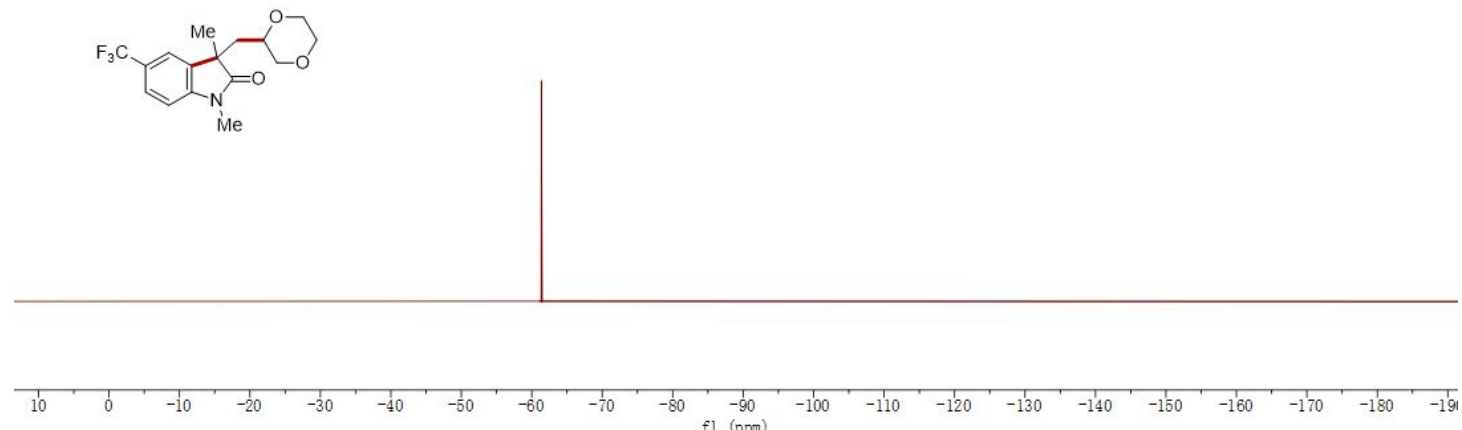

${ }^{1} \mathrm{H}$ NMR of $\mathbf{3 f b}-2\left(500 \mathrm{MHz}, \mathrm{CDCl}_{3}\right)$

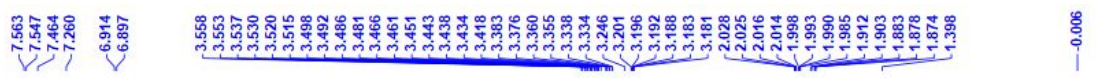
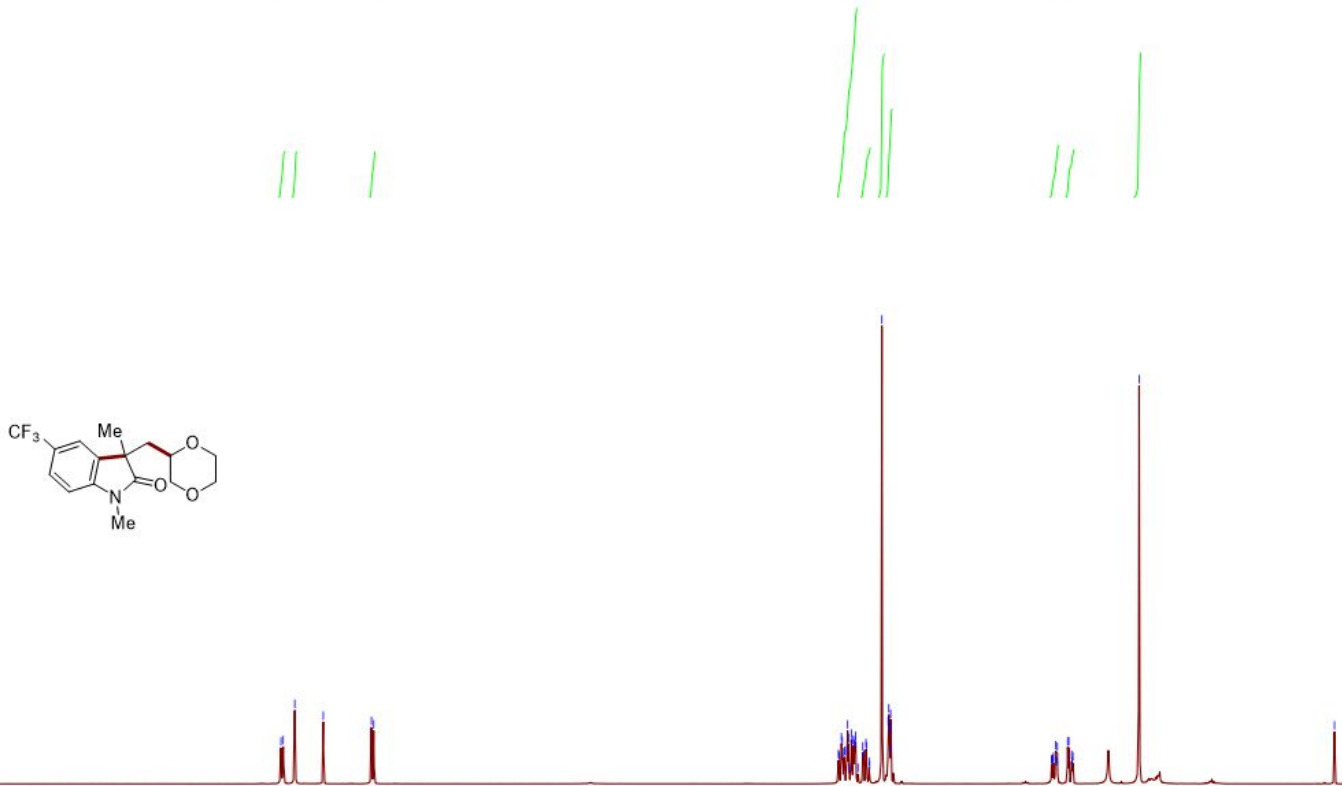

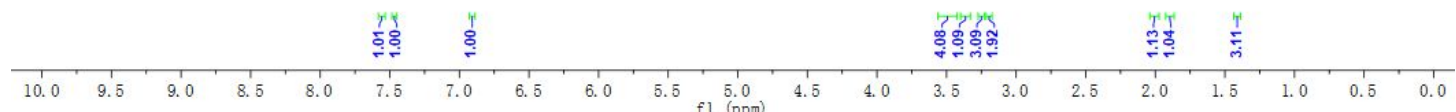




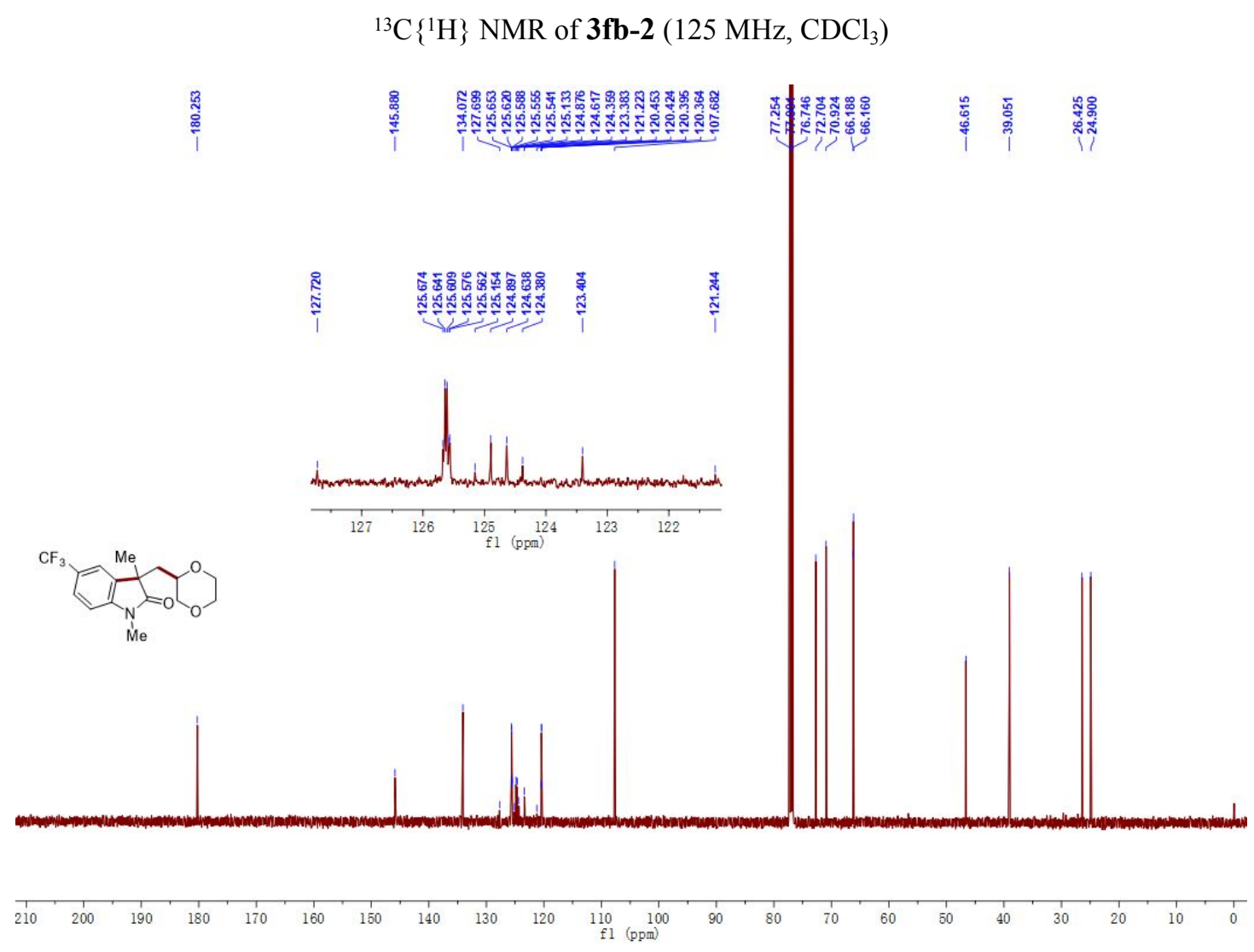

${ }^{19} \mathrm{~F}$ NMR of $\mathbf{3 f b}-\mathbf{2}\left(565 \mathrm{MHz}, \mathrm{CDCl}_{3}\right)$

$\frac{2}{1}$
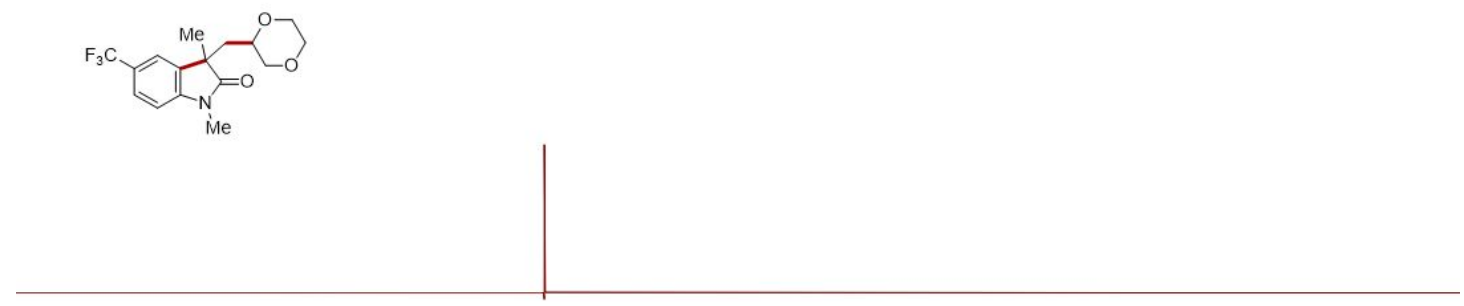

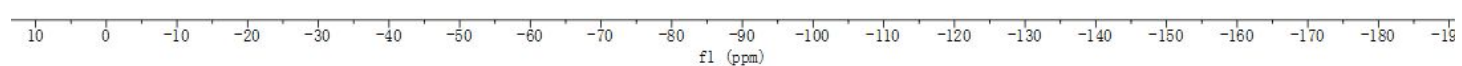


${ }^{1} \mathrm{H}$ NMR of 3gb-1 (500 MHz, $\left.\mathrm{CDCl}_{3}\right)$

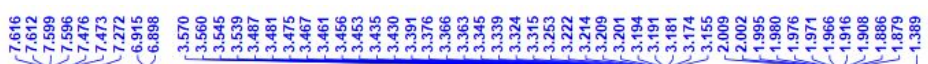
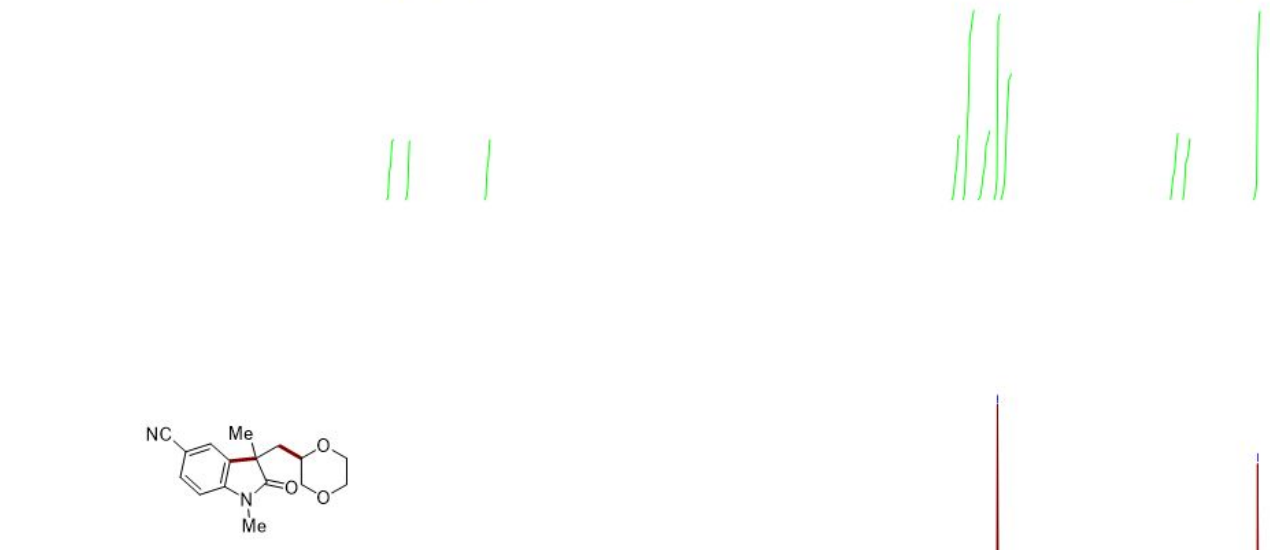

$\pi \int$

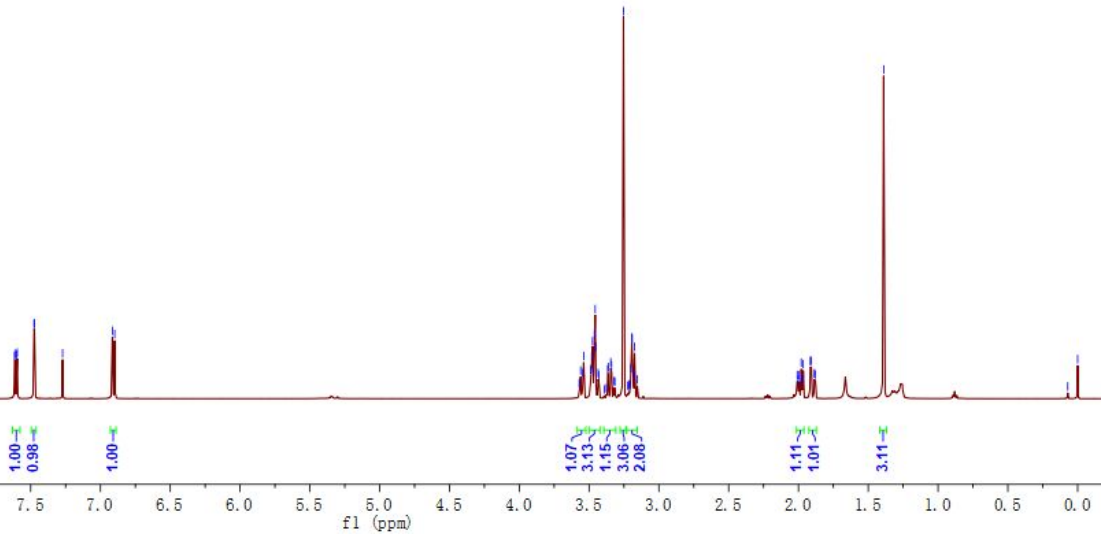

${ }^{13} \mathrm{C}\left\{{ }^{1} \mathrm{H}\right\}$ NMR of $\mathbf{3 g b}-\mathbf{1}\left(125 \mathrm{MHz}, \mathrm{CDCl}_{3}\right)$

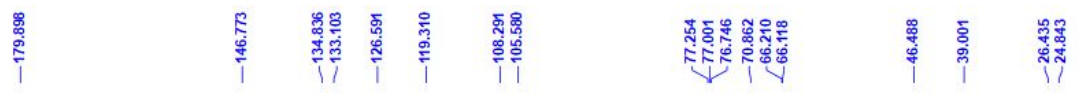
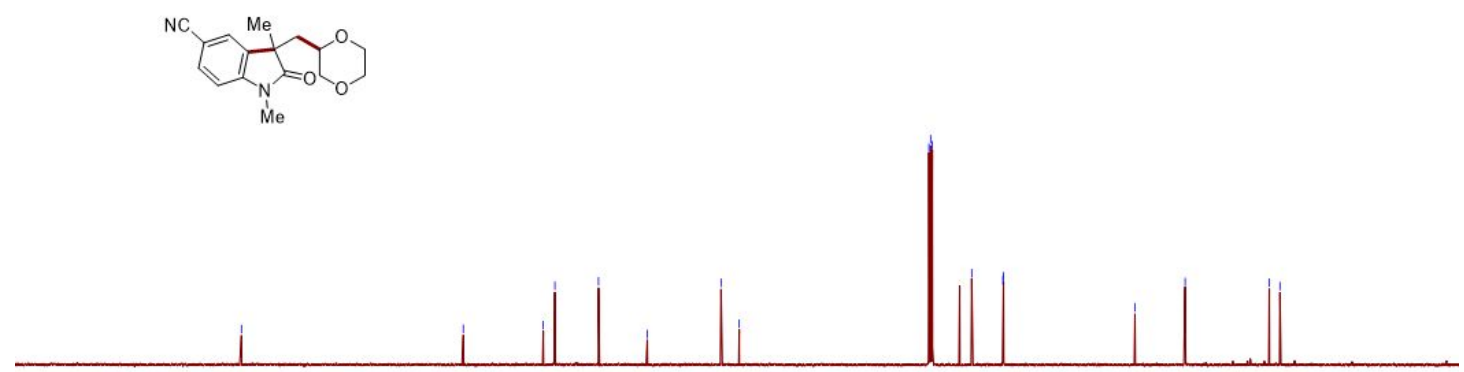

$\begin{array}{lllllllllll}210 & 200 & 190 & 180 & 170 & 160 & 150 & 140 & 130 & 120 & 110 \\ \mathrm{fl}(\mathrm{ppm}) & 100\end{array}$ 
${ }^{1} \mathrm{H}$ NMR of 3gb-2 (500 MHz, $\left.\mathrm{CDCl}_{3}\right)$

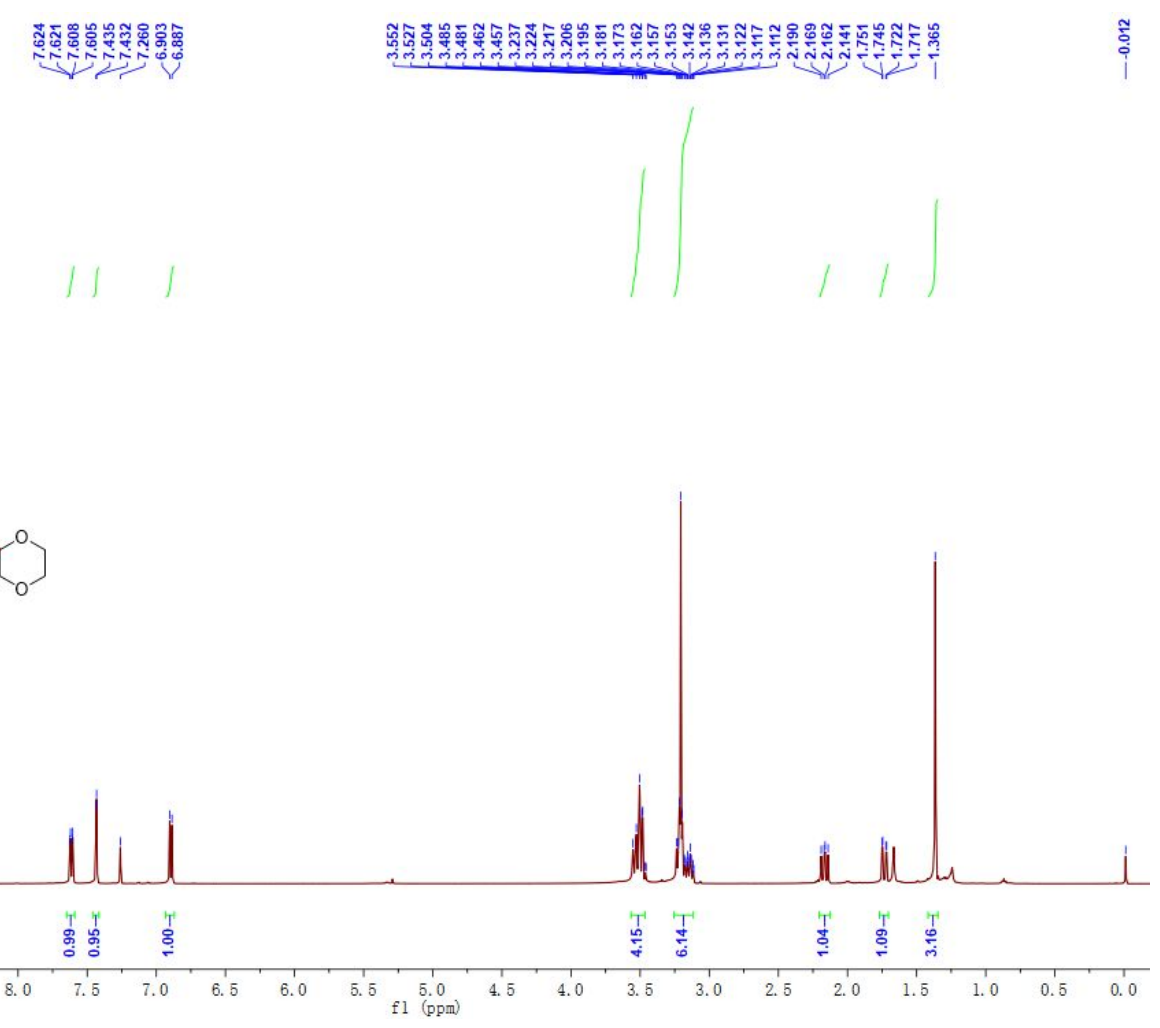

${ }^{13} \mathrm{C}\left\{{ }^{1} \mathrm{H}\right\}$ NMR of $\mathbf{3 g b}-2\left(125 \mathrm{MHz}, \mathrm{CDCl}_{3}\right)$

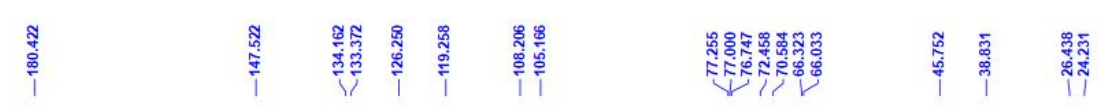<smiles></smiles>
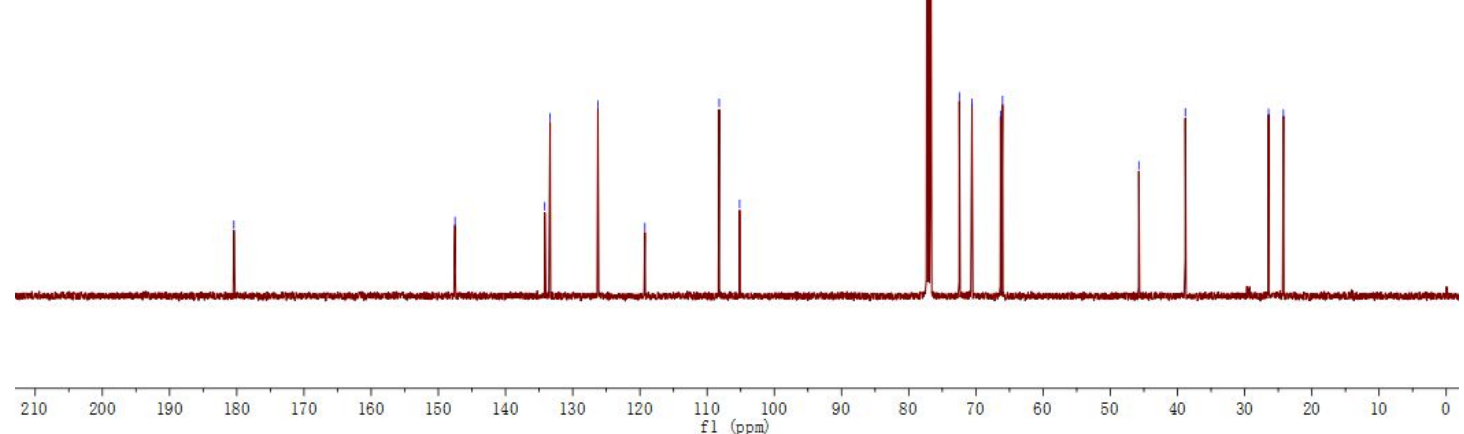
${ }^{1} \mathrm{H}$ NMR of $3 g a\left(500 \mathrm{MHz}, \mathrm{CDCl}_{3}\right)$

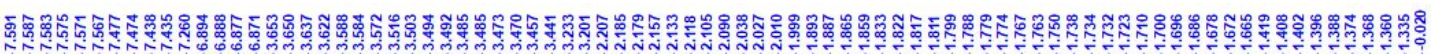

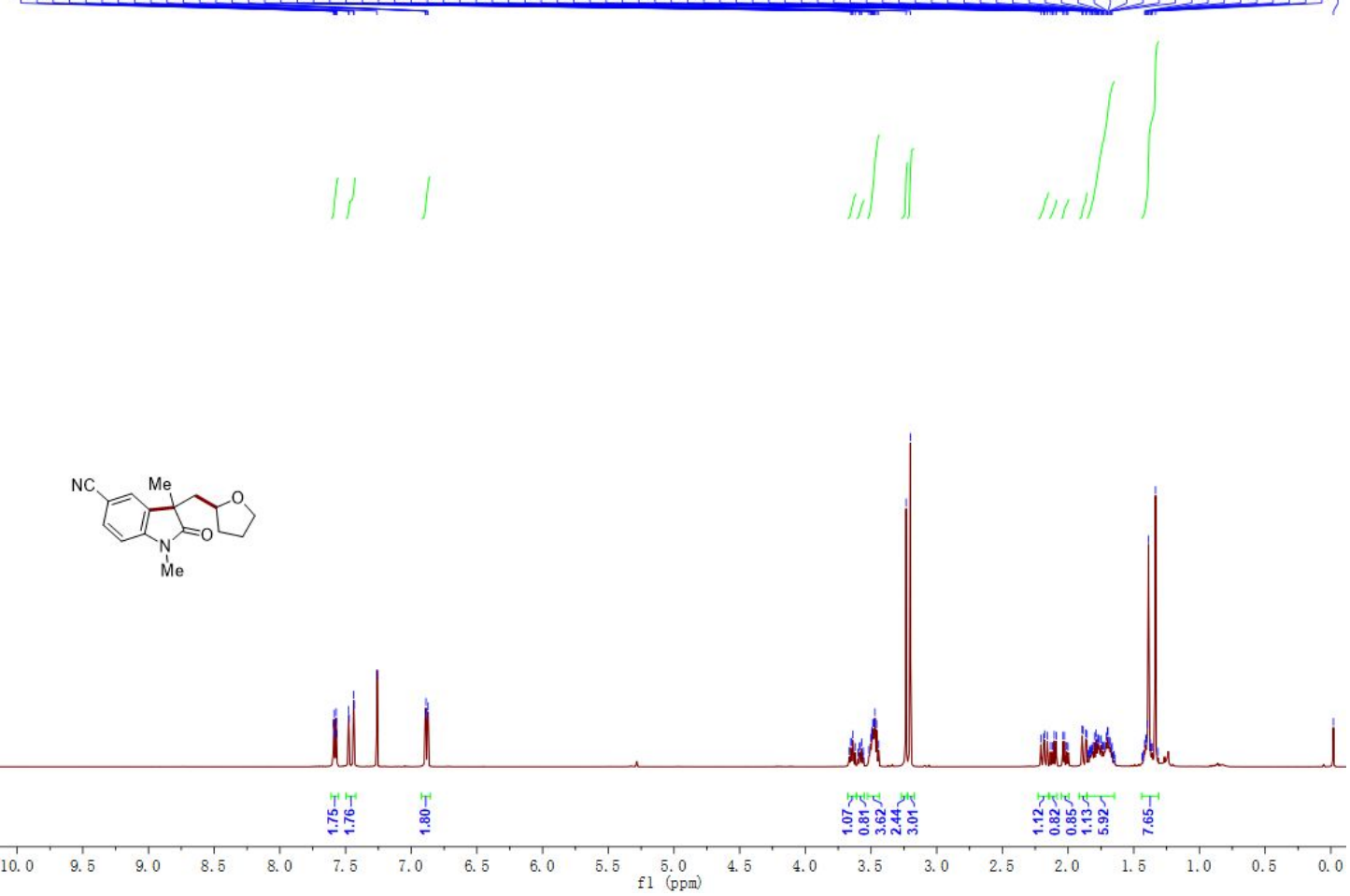

${ }^{13} \mathrm{C}\left\{{ }^{1} \mathrm{H}\right\} \mathrm{NMR}$ of 3 ga $\left(125 \mathrm{MHz}, \mathrm{CDCl}_{3}\right)$

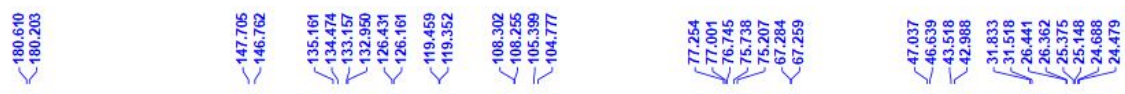

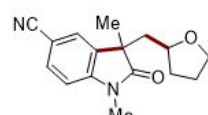

Me

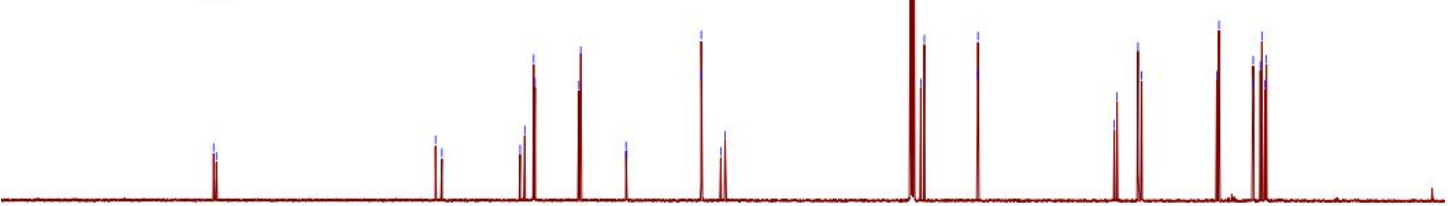

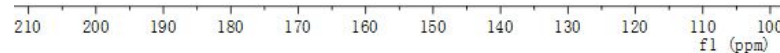


${ }^{1} \mathrm{H}$ NMR of (3hb + 3hb')-1 (500 MHz, $\left.\mathrm{CDCl}_{3}\right)$

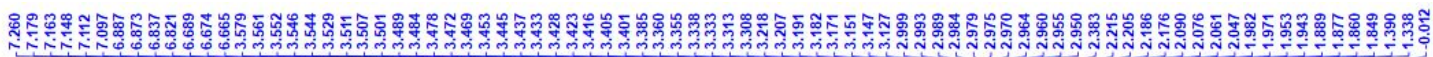
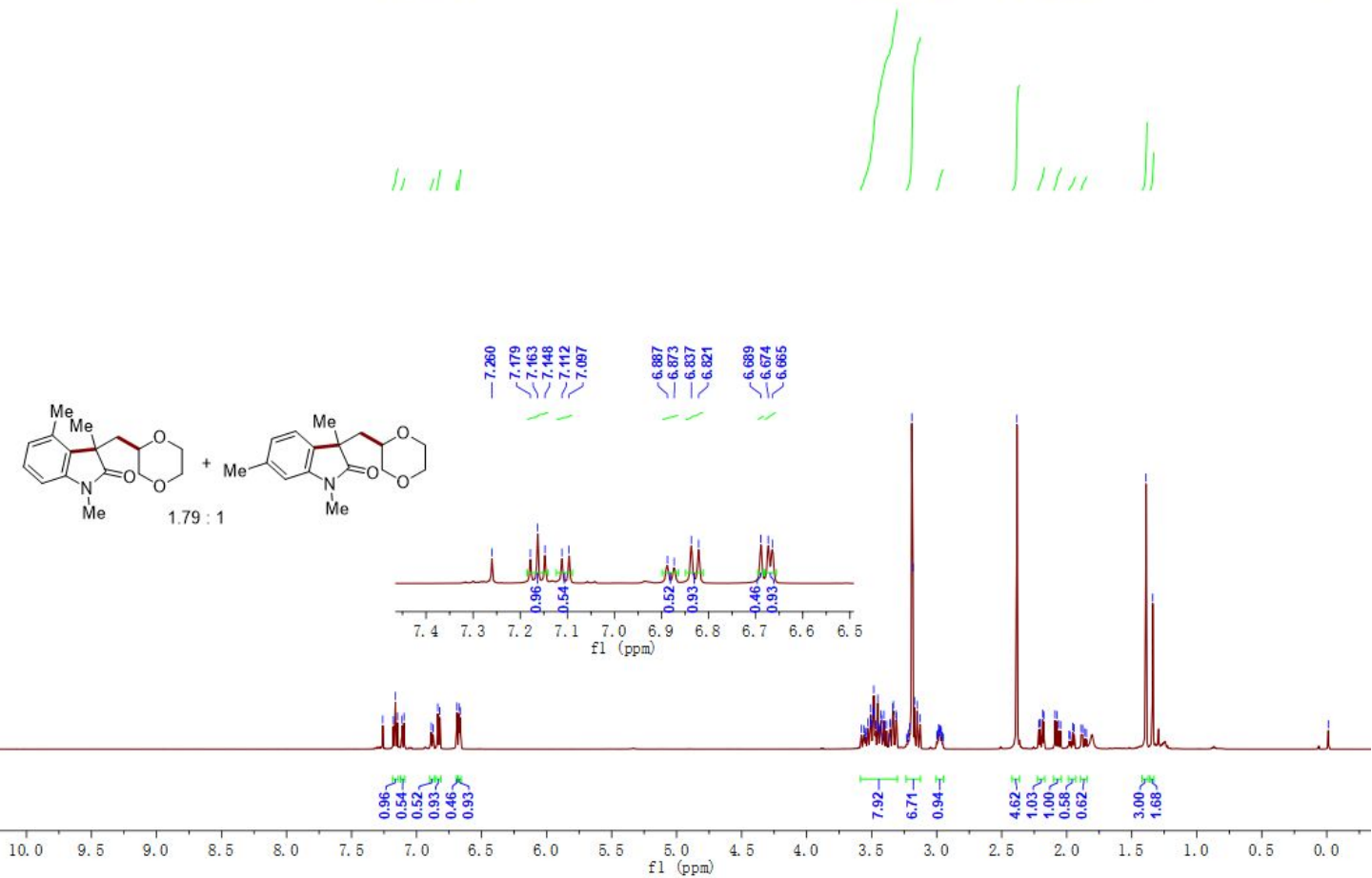

${ }^{13} \mathrm{C}\left\{{ }^{1} \mathrm{H}\right\}$ NMR of (3hb $\left.+\mathbf{3 h} \mathbf{b}^{\prime}\right)-\mathbf{1}\left(125 \mathrm{MHz}, \mathrm{CDCl}_{3}\right)$

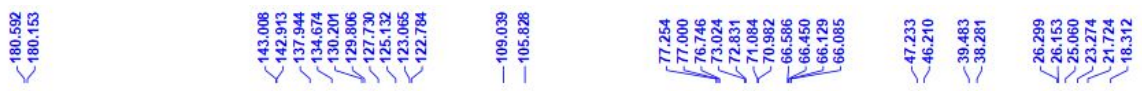
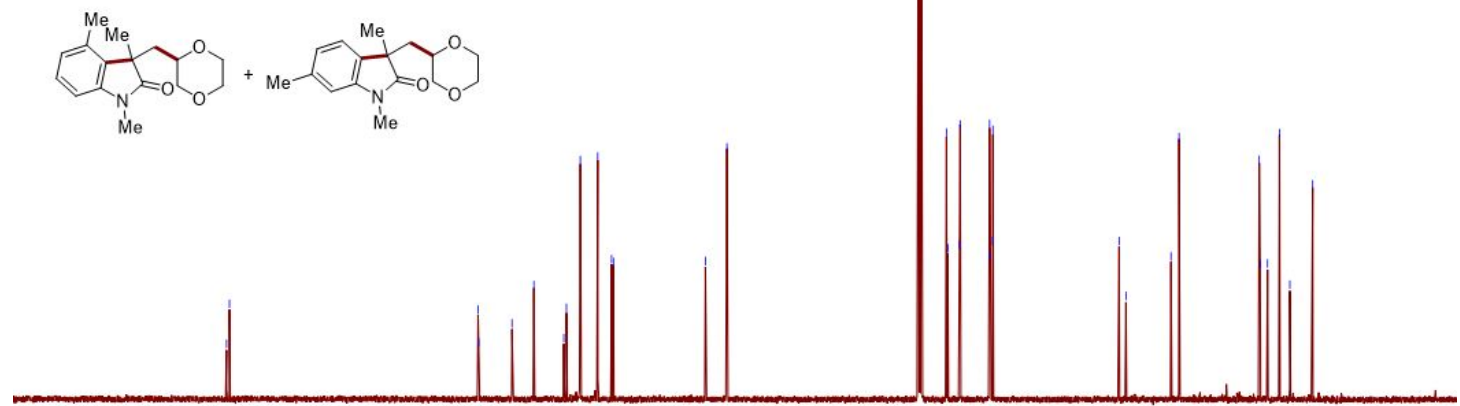

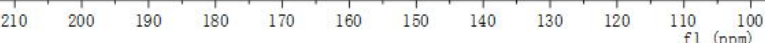

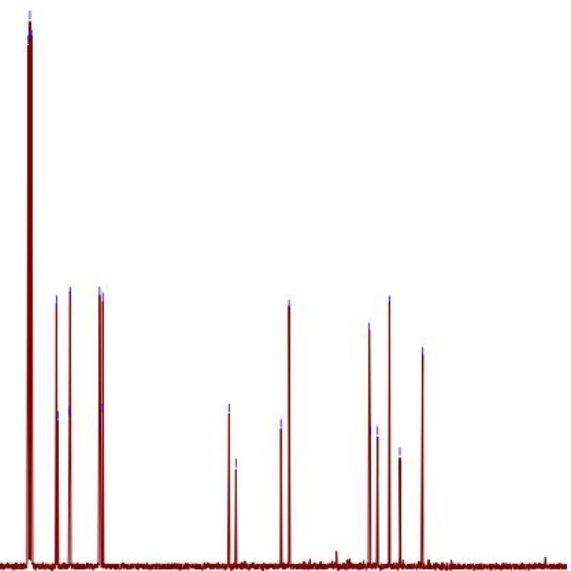


${ }^{1} \mathrm{H}$ NMR of (3hb $\left.+\mathbf{3 h} \mathbf{b}^{\prime}\right)-\mathbf{2}\left(500 \mathrm{MHz}, \mathrm{CDCl}_{3}\right)$

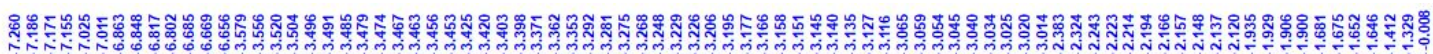

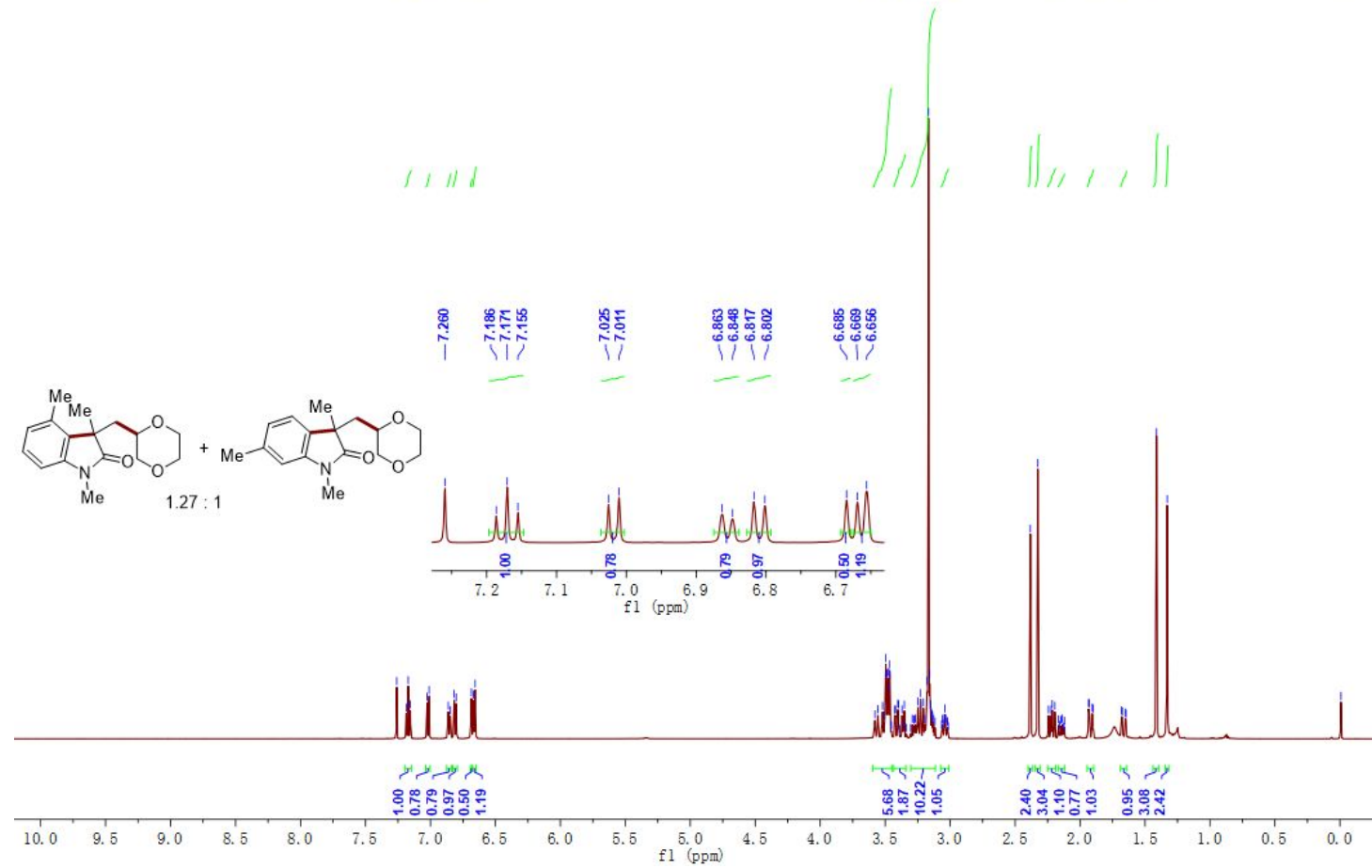

${ }^{13} \mathrm{C}\left\{{ }^{1} \mathrm{H}\right\}$ NMR of (3hb $\left.+\mathbf{3 h} \mathbf{b}^{\prime}\right)-\mathbf{2}\left(125 \mathrm{MHz}, \mathrm{CDCl}_{3}\right)$

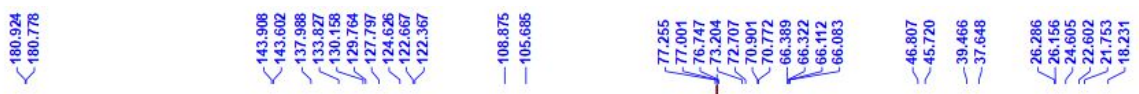

$\underbrace{M e}_{M=0}+C_{M e}^{M}$

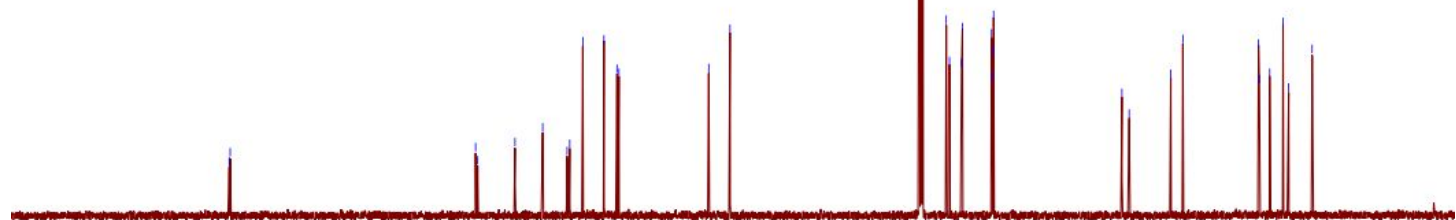

$\begin{array}{llllllllllll}210 & 200 & 190 & 180 & 170 & 160 & 150 & 140 & 130 & 120 & 110 & 100\end{array}$ 
${ }^{1} \mathrm{H} \mathrm{NMR}$ of (3ib + 3ib')-1 (500 MHz, $\left.\mathrm{CDCl}_{3}\right)$

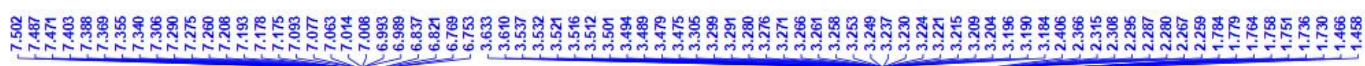
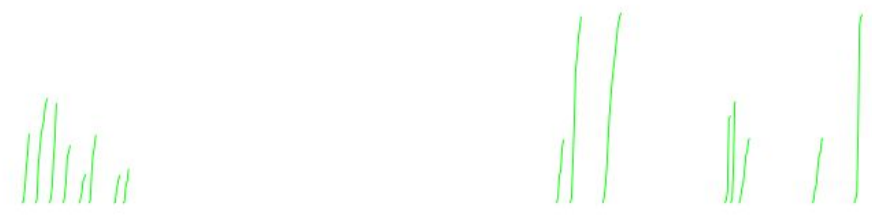

Pe

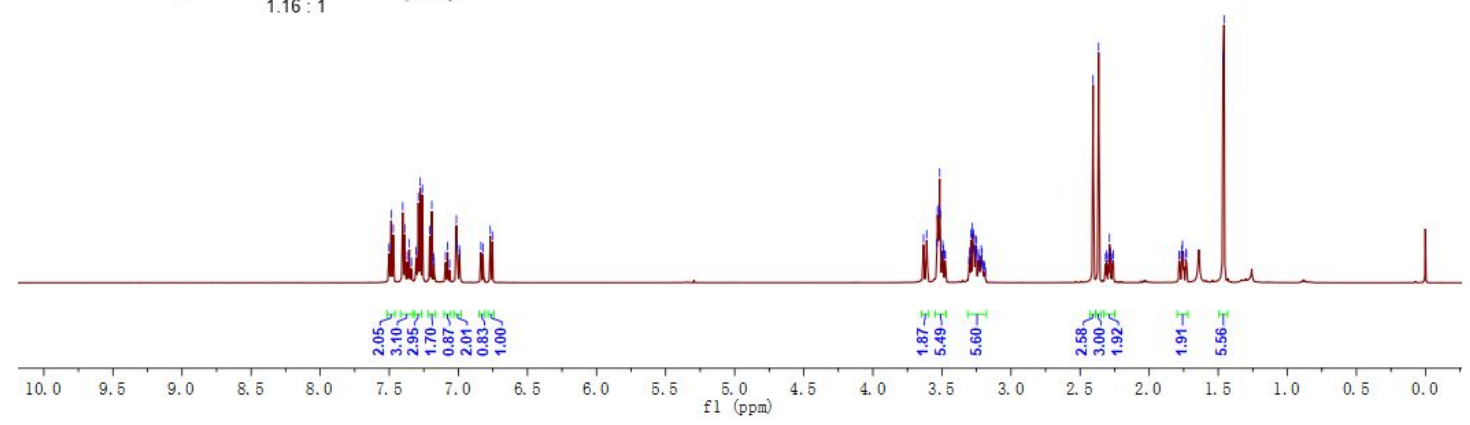

${ }^{13} \mathrm{C}\left\{{ }^{1} \mathrm{H}\right\}$ NMR of (3ib + 3ib')-1 (125 MHz, $\left.\mathrm{CDCl}_{3}\right)$

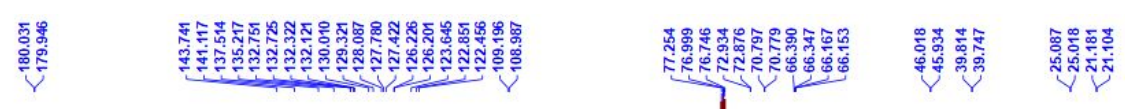

Me

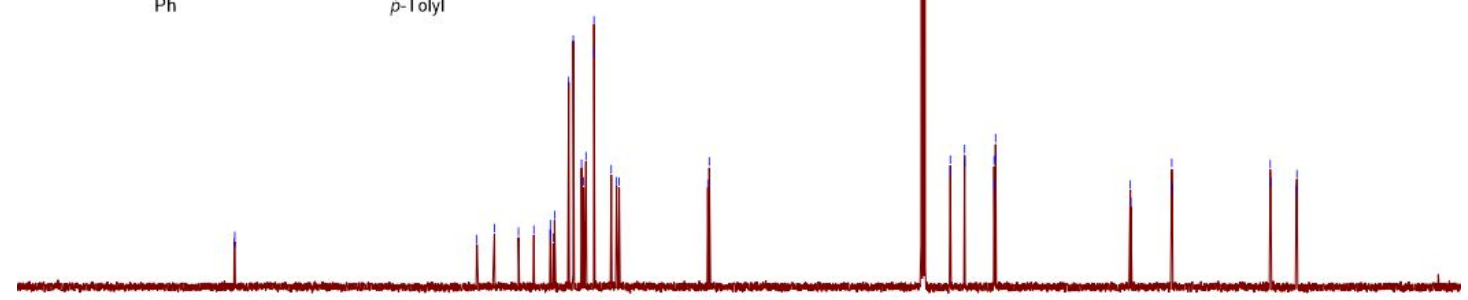

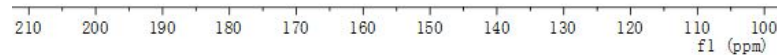


${ }^{1} \mathrm{H} \mathrm{NMR}$ of (3ib + 3ib')-2 (500 MHz, $\left.\mathrm{CDCl}_{3}\right)$

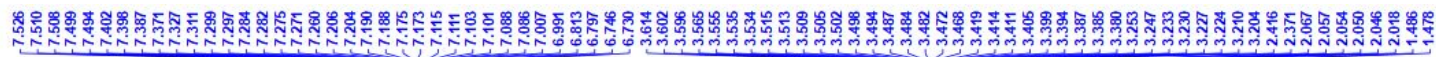

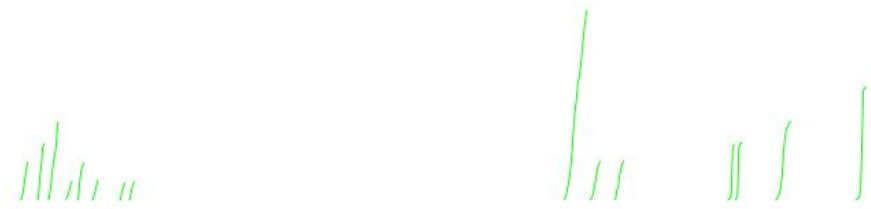

1.04:1

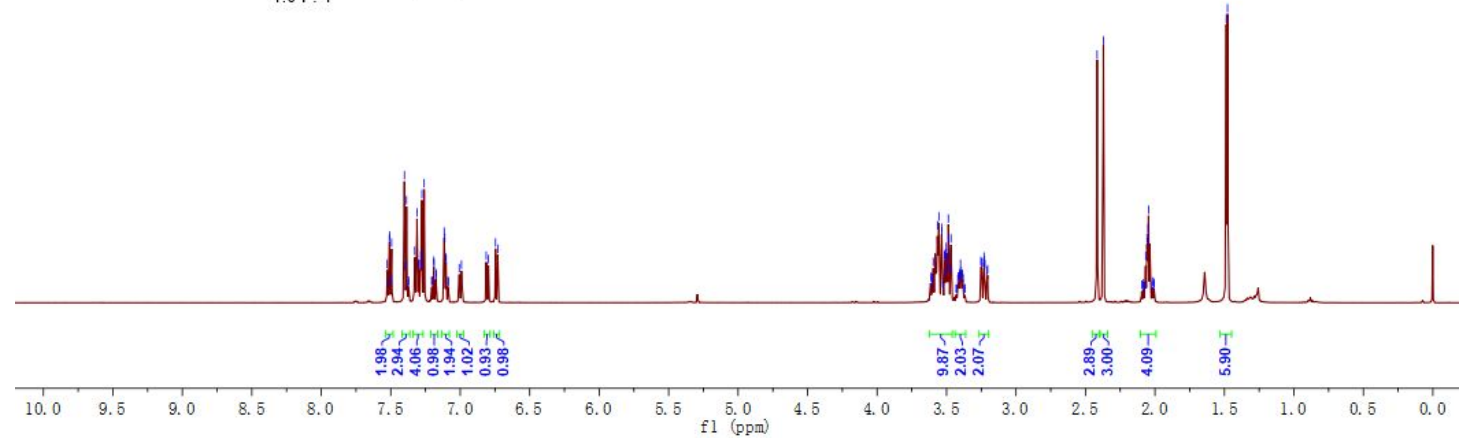

${ }^{13} \mathrm{C}\left\{{ }^{1} \mathrm{H}\right\}$ NMR of (3ib + 3ib')-2 (125 MHz, $\left.\mathrm{CDCl}_{3}\right)$

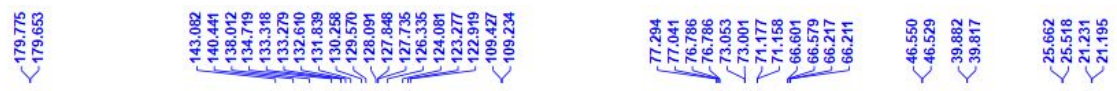

Me

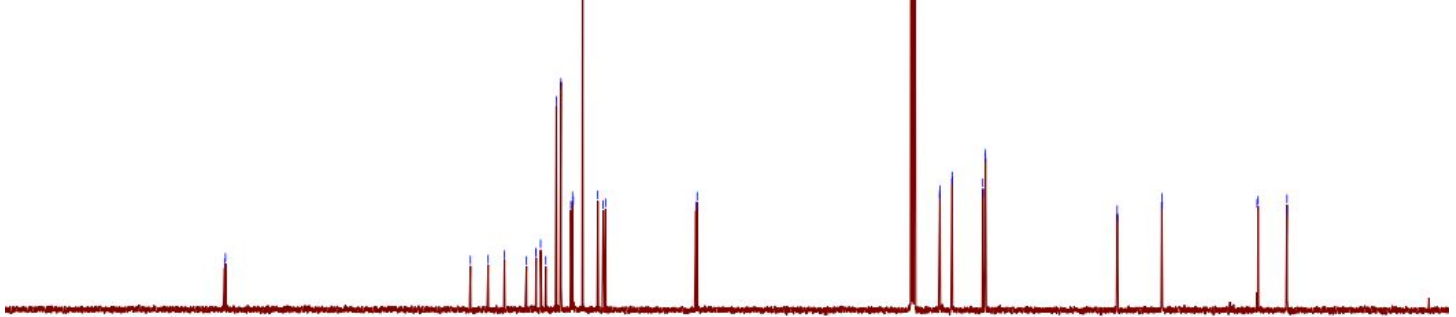

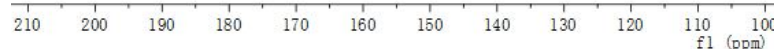


${ }^{1} \mathrm{H}$ NMR of $\mathbf{3 j b}-\mathbf{1}\left(500 \mathrm{MHz}, \mathrm{CDCl}_{3}\right)$

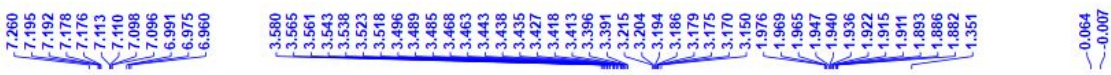

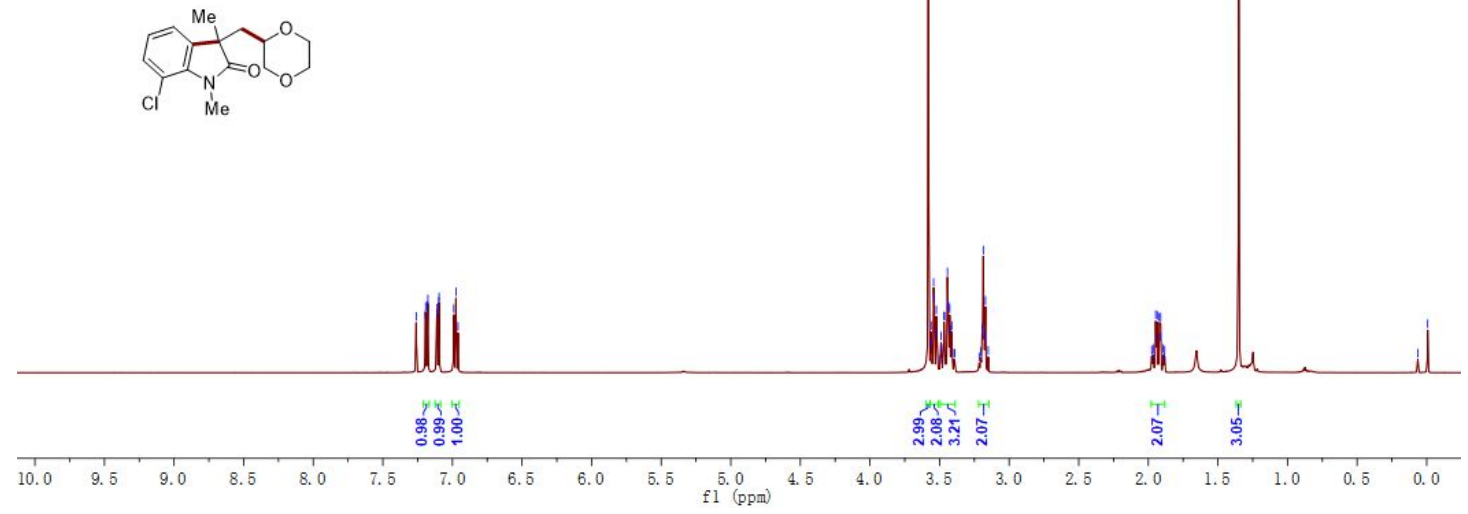

${ }^{13} \mathrm{C}\left\{{ }^{1} \mathrm{H}\right\}$ NMR of $\mathbf{3 j b}-\mathbf{1}\left(125 \mathrm{MHz}, \mathrm{CDCl}_{3}\right)$

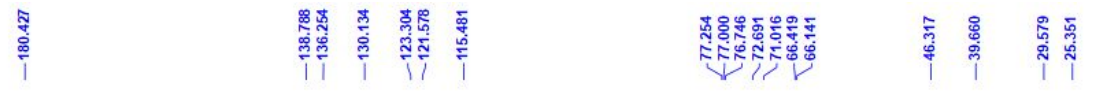

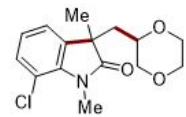

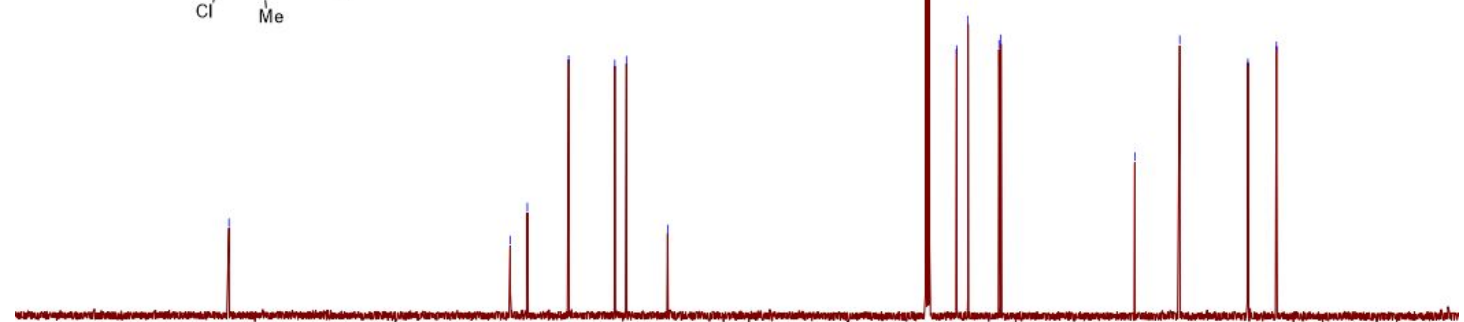

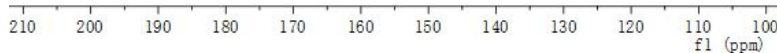

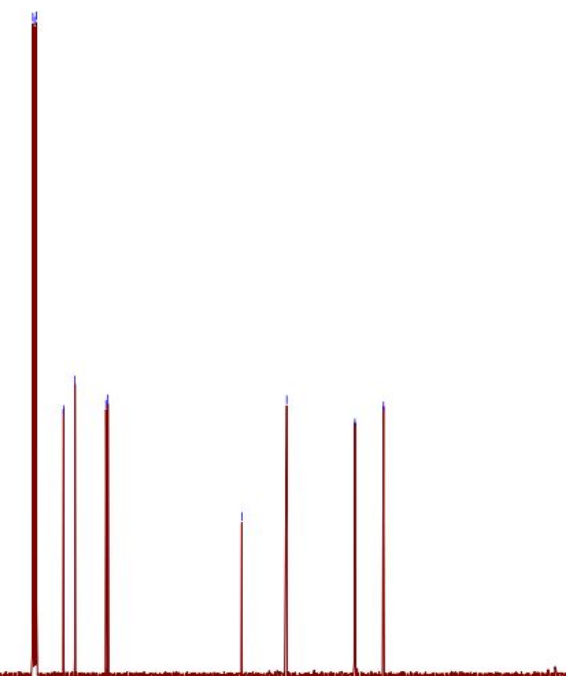


${ }^{1} \mathrm{H}$ NMR of $\mathbf{3 j b}-2\left(500 \mathrm{MHz}, \mathrm{CDCl}_{3}\right)$

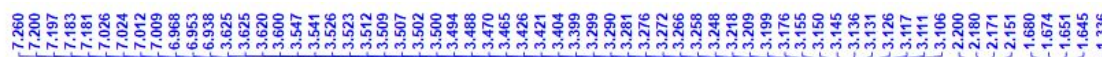

$\stackrel{8}{8}$

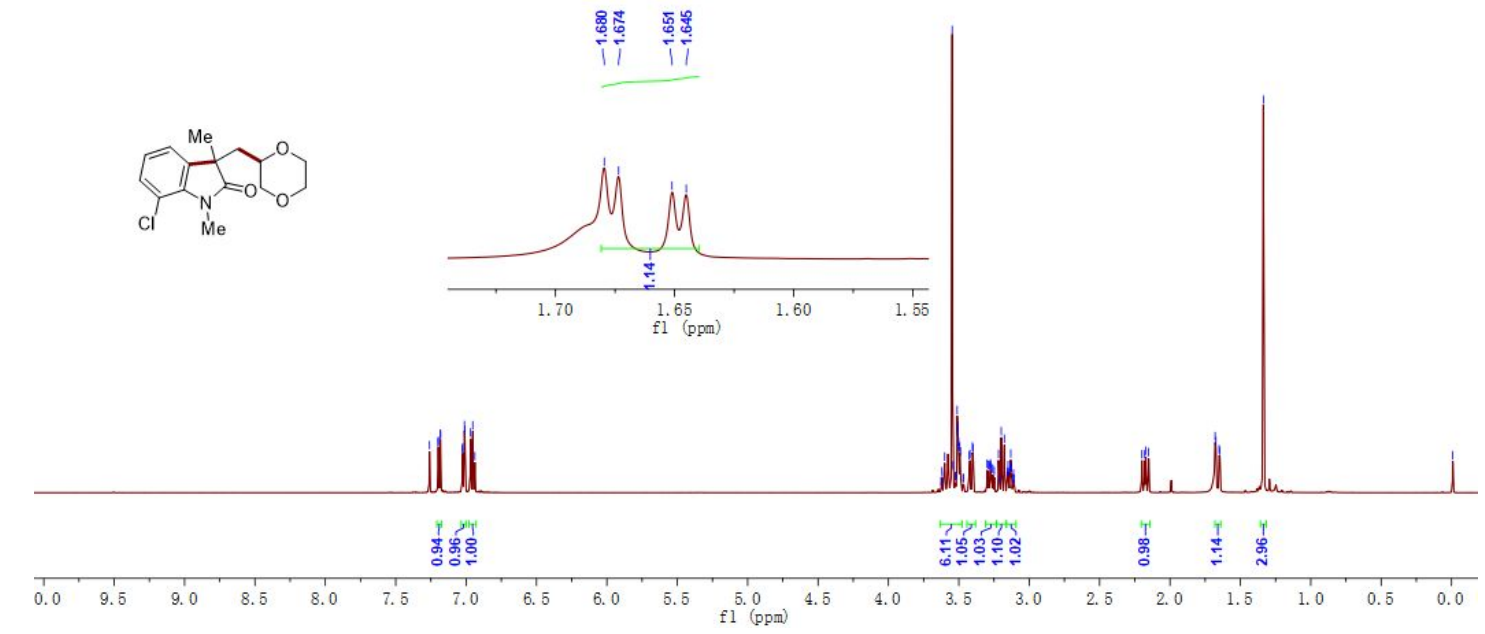

${ }^{13} \mathrm{C}\left\{{ }^{1} \mathrm{H}\right\}$ NMR of $\mathbf{3 j b}-\mathbf{2}\left(125 \mathrm{MHz}, \mathrm{CDCl}_{3}\right)$

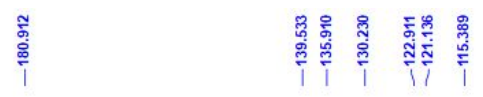

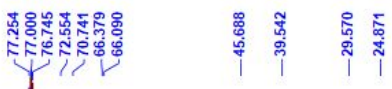
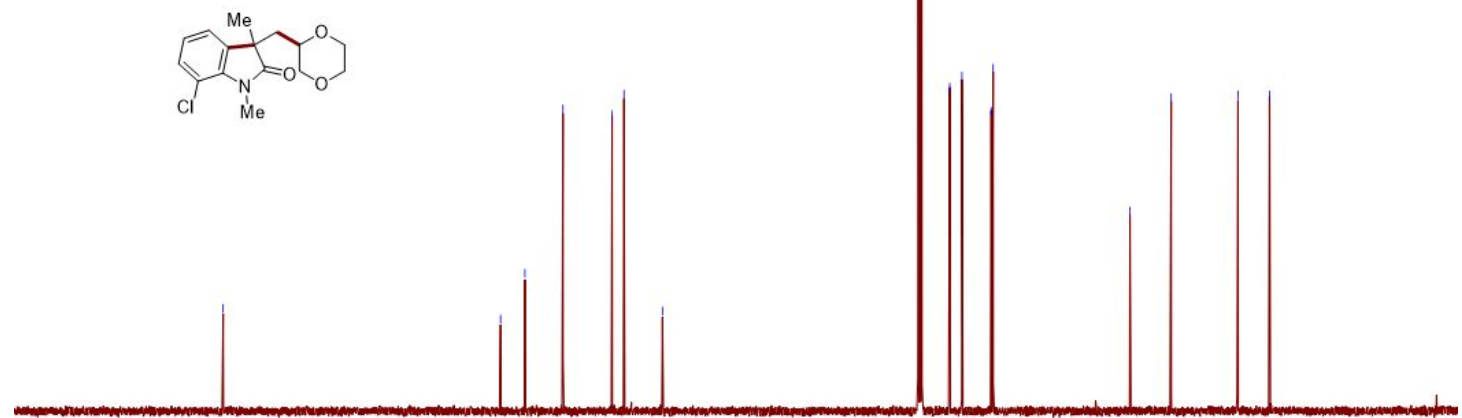

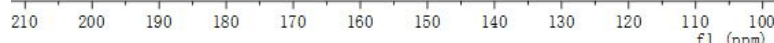


${ }^{1} \mathrm{H}$ NMR of $\mathbf{3 k b}-\mathbf{1}\left(500 \mathrm{MHz}, \mathrm{CDCl}_{3}\right)$

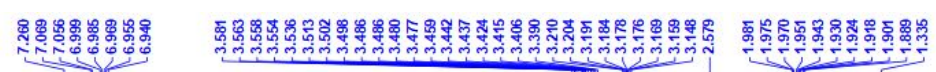
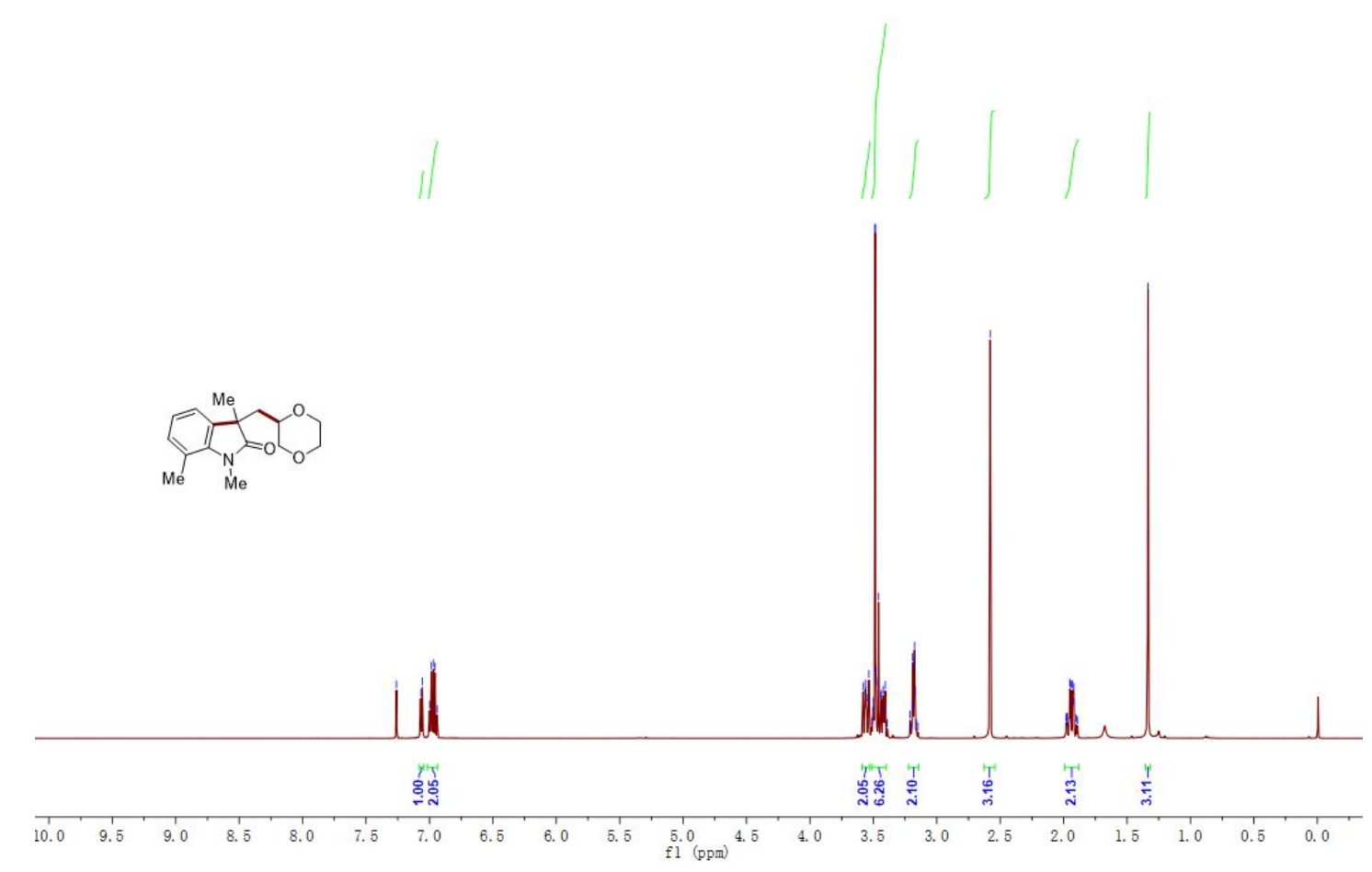

${ }^{13} \mathrm{C}\left\{{ }^{1} \mathrm{H}\right\}$ NMR of $\mathbf{3 k b}-\mathbf{1}\left(125 \mathrm{MHz}, \mathrm{CDCl}_{3}\right)$

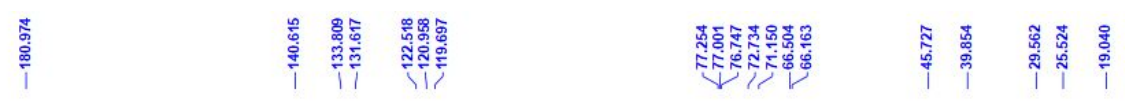
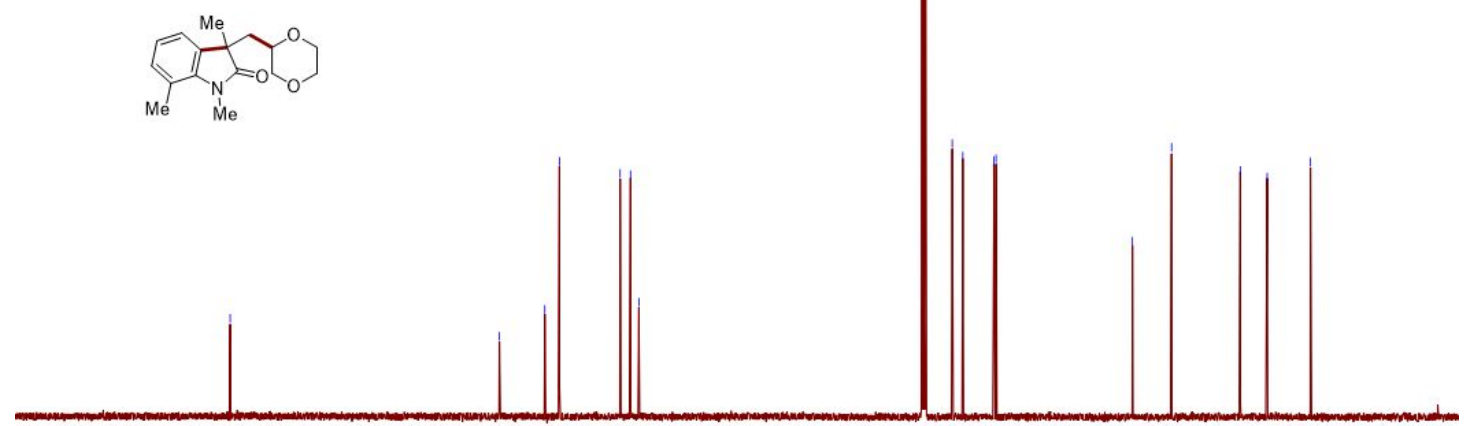

$\begin{array}{llllllllllll}210 & 200 & 190 & 180 & 170 & 160 & 150 & 140 & 130 & 120 & 110 & 100 \\ \mathrm{fl} & (\mathrm{ppm})\end{array}$ 
${ }^{1} \mathrm{H}$ NMR of $\mathbf{3 k b}-2\left(500 \mathrm{MHz}, \mathrm{CDCl}_{3}\right)$

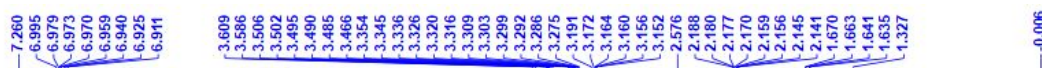

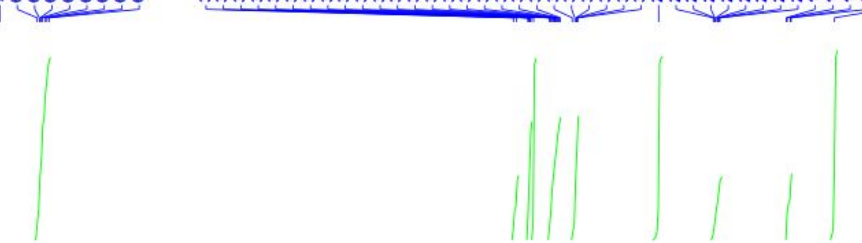

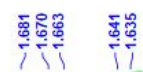

$\sum_{\mathrm{Me}}^{\mathrm{Me}=0}$
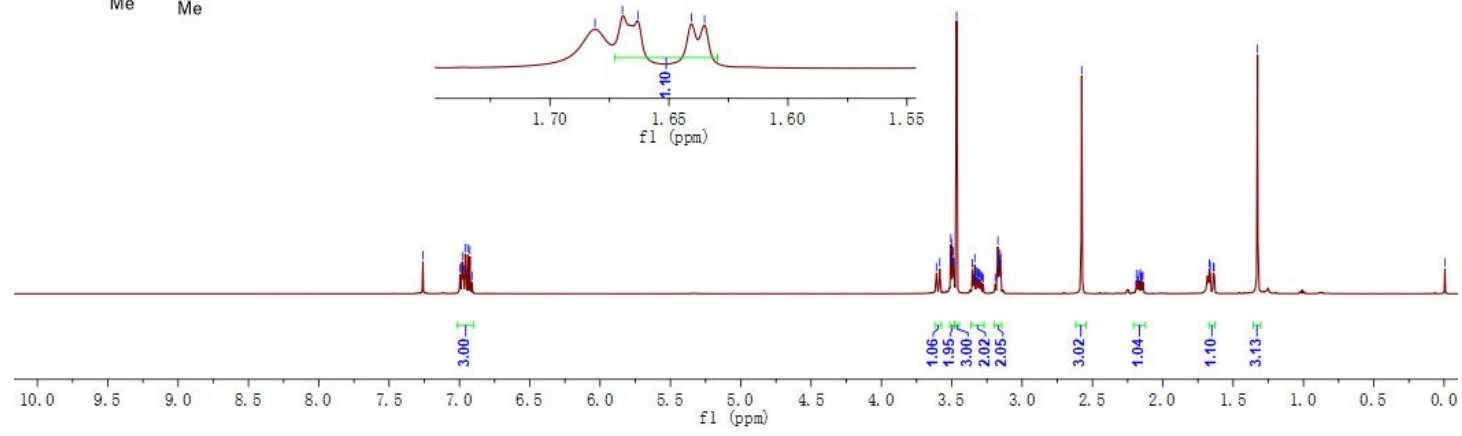

${ }^{13} \mathrm{C}\left\{{ }^{1} \mathrm{H}\right\}$ NMR of $\mathbf{3 k b}-2\left(125 \mathrm{MHz}, \mathrm{CDCl}_{3}\right)$

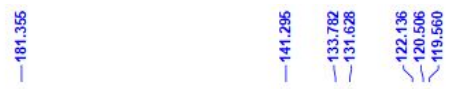

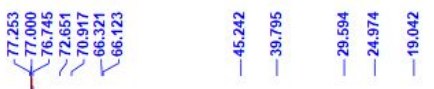
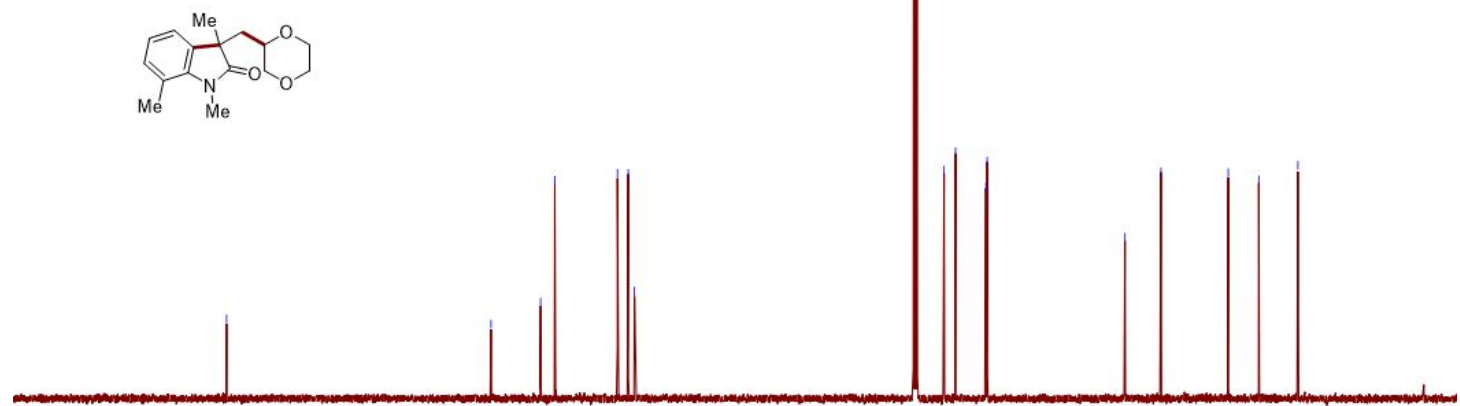

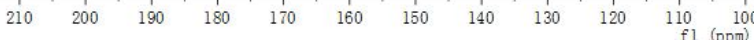

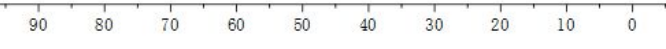


${ }^{1} \mathrm{H}$ NMR of 3la $\left(500 \mathrm{MHz}, \mathrm{CDCl}_{3}\right)$

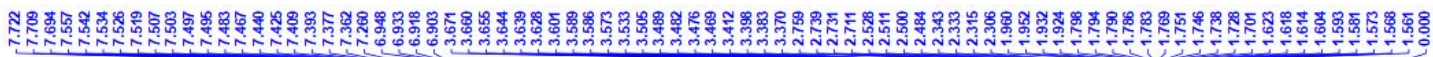

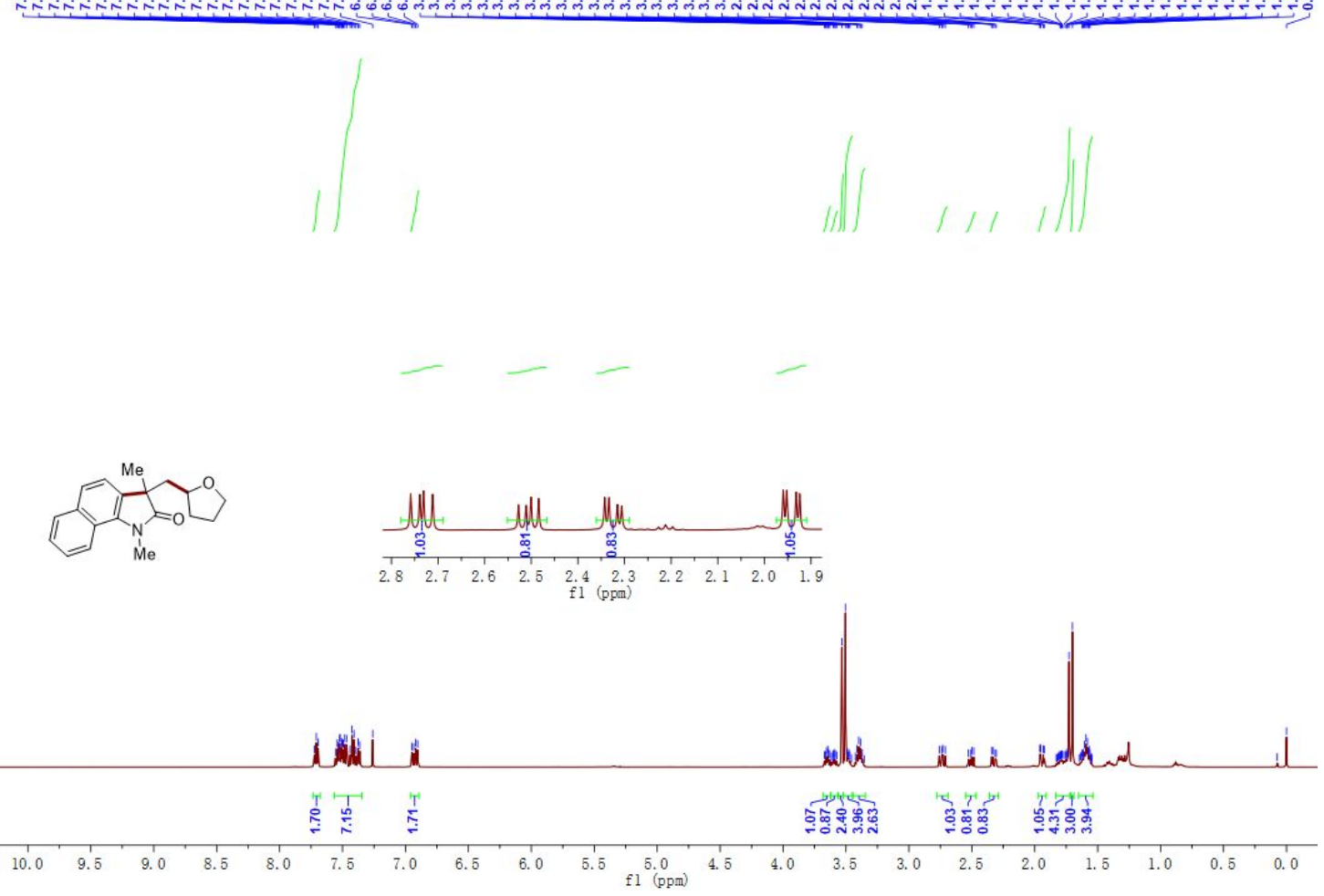

${ }^{13} \mathrm{C}\left\{{ }^{1} \mathrm{H}\right\}$ NMR of 3 la $\left(125 \mathrm{MHz}, \mathrm{CDCl}_{3}\right)$
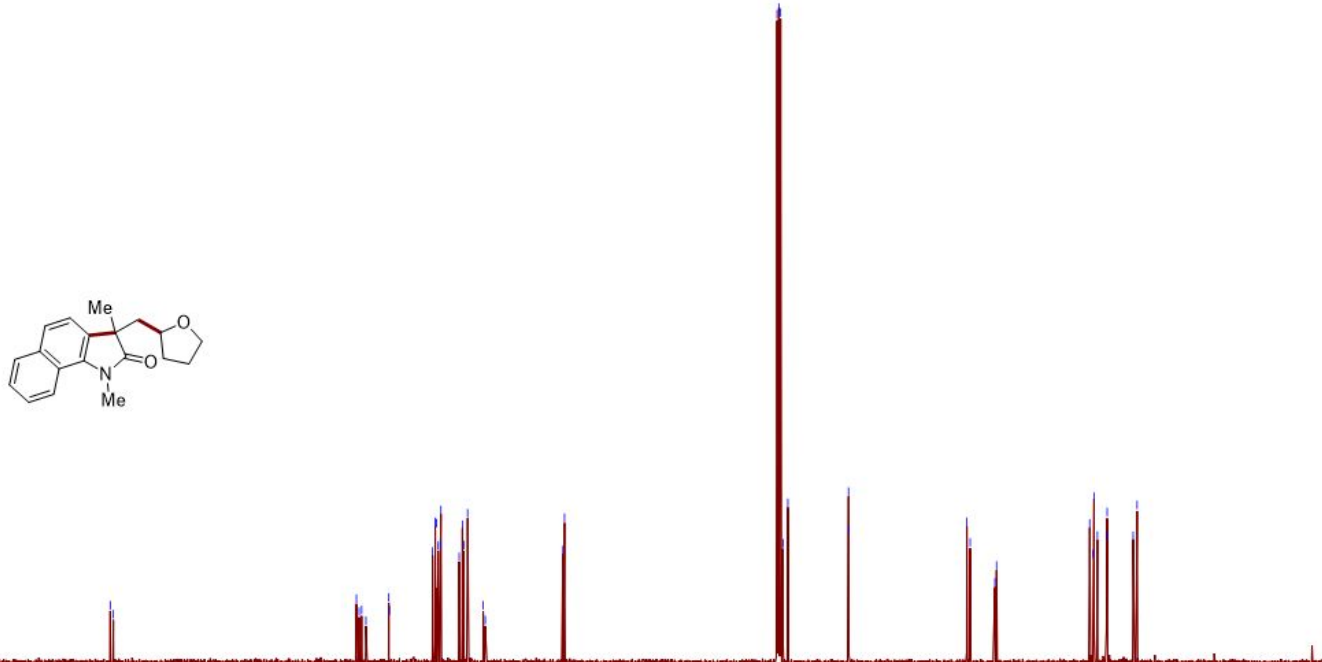

$\begin{array}{lllllllllll}200 & 190 & 180 & 170 & 160 & 150 & 140 & 130 & 120 & 110 & 100\end{array}$

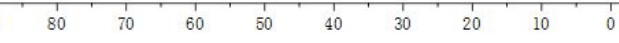


${ }^{1} \mathrm{H}$ NMR of 3mb-1 (500 MHz, $\left.\mathrm{CDCl}_{3}\right)$

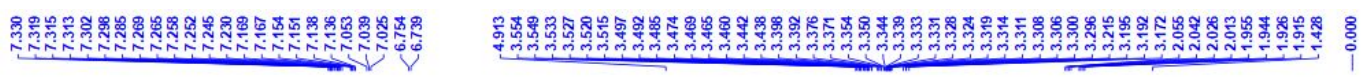

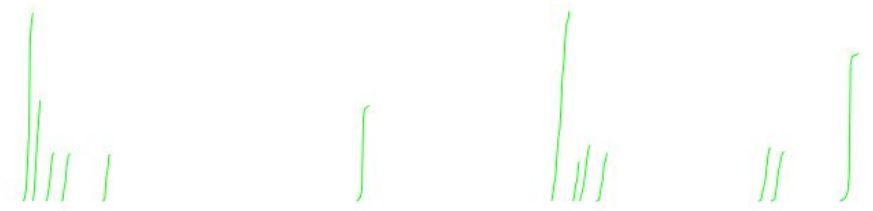

$\sum_{\substack{N \\ B n}}^{M e}=0$

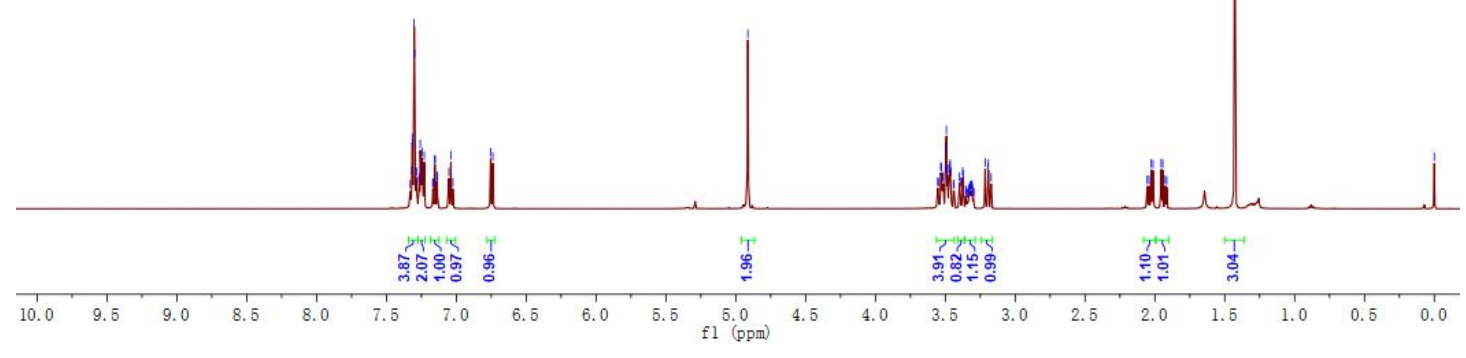

${ }^{13} \mathrm{C}\left\{{ }^{1} \mathrm{H}\right\}$ NMR of $\mathbf{3 m b}-\mathbf{1}\left(125 \mathrm{MHz}, \mathrm{CDCl}_{3}\right)$

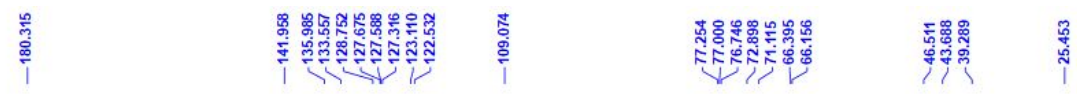

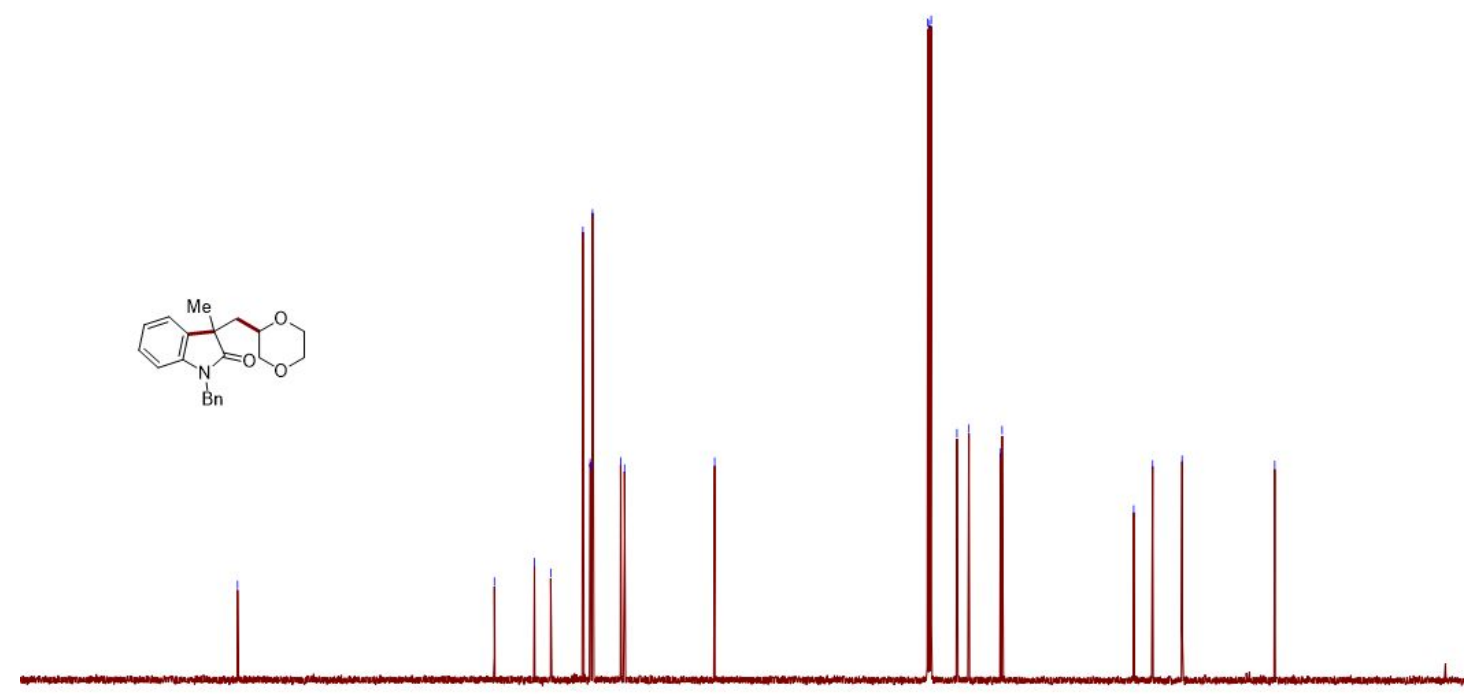

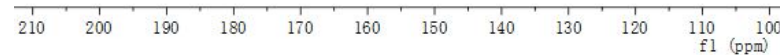


${ }^{1} \mathrm{H} \mathrm{NMR}$ of $\mathbf{3 m b}-2\left(500 \mathrm{MHz}, \mathrm{CDCl}_{3}\right)$

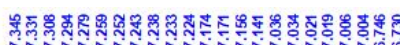

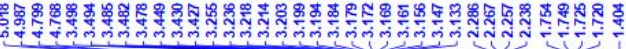
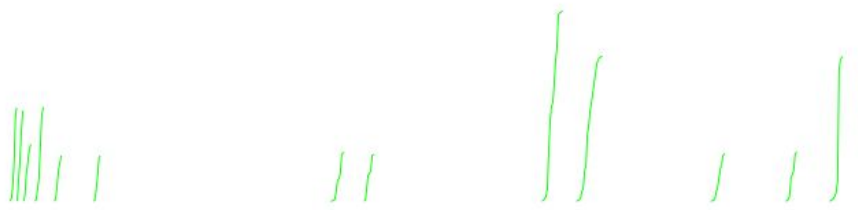

$\overbrace{n}^{M e}=C_{0}^{O}$

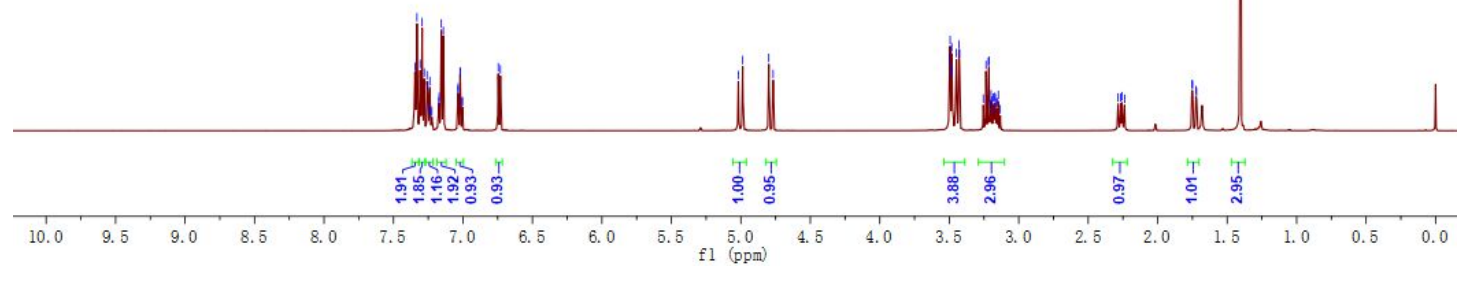

${ }^{13} \mathrm{C}\left\{{ }^{1} \mathrm{H}\right\}$ NMR of $\mathbf{3 m b}-\mathbf{2}\left(125 \mathrm{MHz}, \mathrm{CDCl}_{3}\right)$

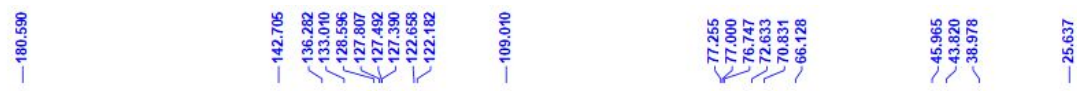

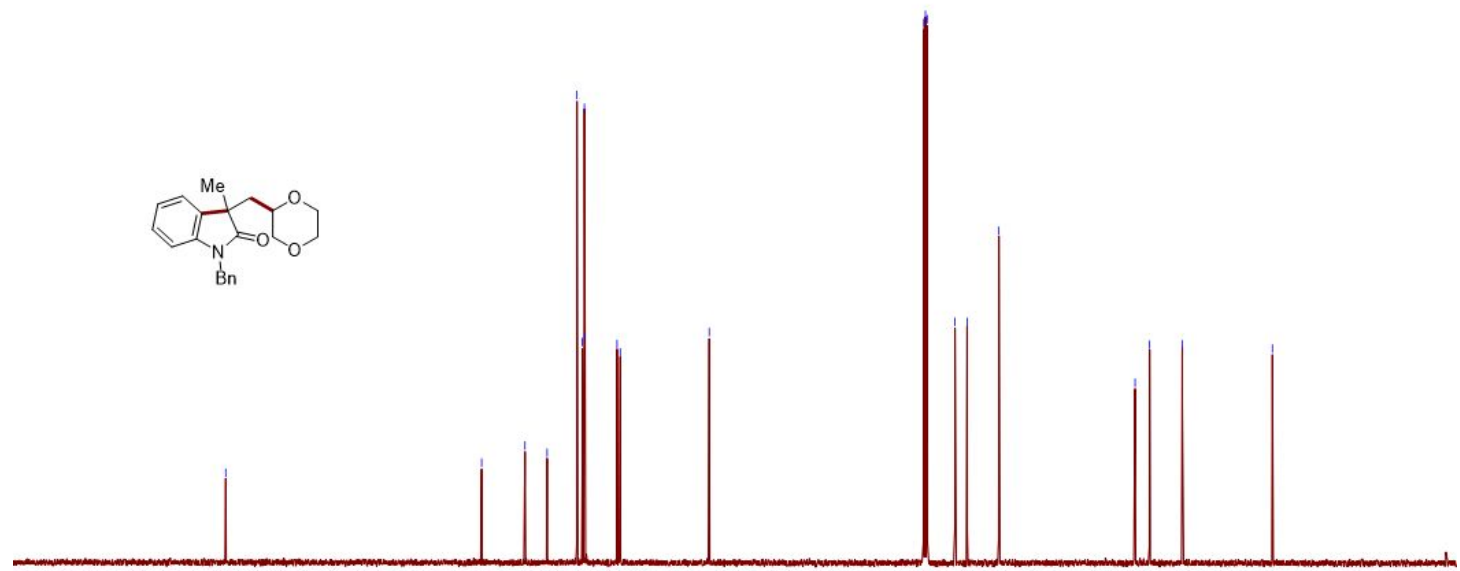

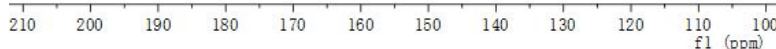


${ }^{1} \mathrm{H}$ NMR of 3nb-1 (500 MHz, $\left.\mathrm{CDCl}_{3}\right)$

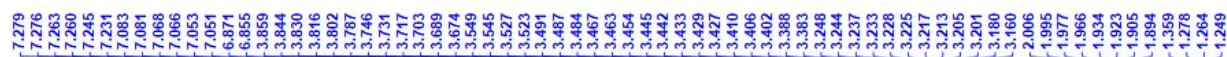

รั.

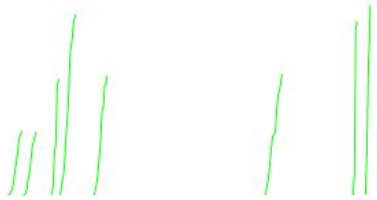

$\underbrace{\mathrm{Me}}_{\substack{n \\ \text { Et }}} \mathrm{O}_{0}^{\mathrm{O}}$

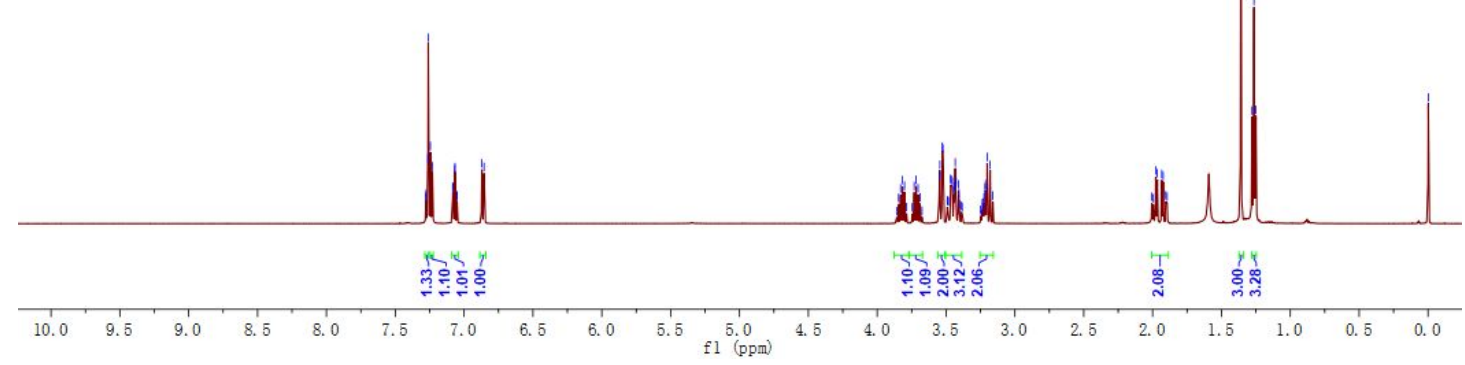

${ }^{13} \mathrm{C}\left\{{ }^{1} \mathrm{H}\right\}$ NMR of $\mathbf{3 n b}-\mathbf{1}\left(125 \mathrm{MHz}, \mathrm{CDCl}_{3}\right)$

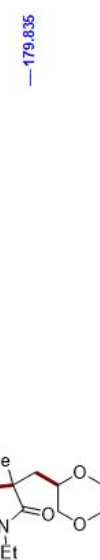

尊

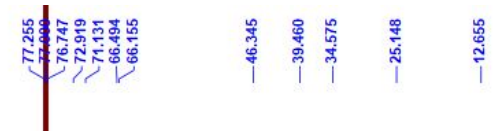

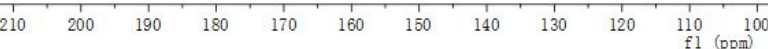


${ }^{1} \mathrm{H}$ NMR of 3nb-2 (500 MHz, $\left.\mathrm{CDCl}_{3}\right)$

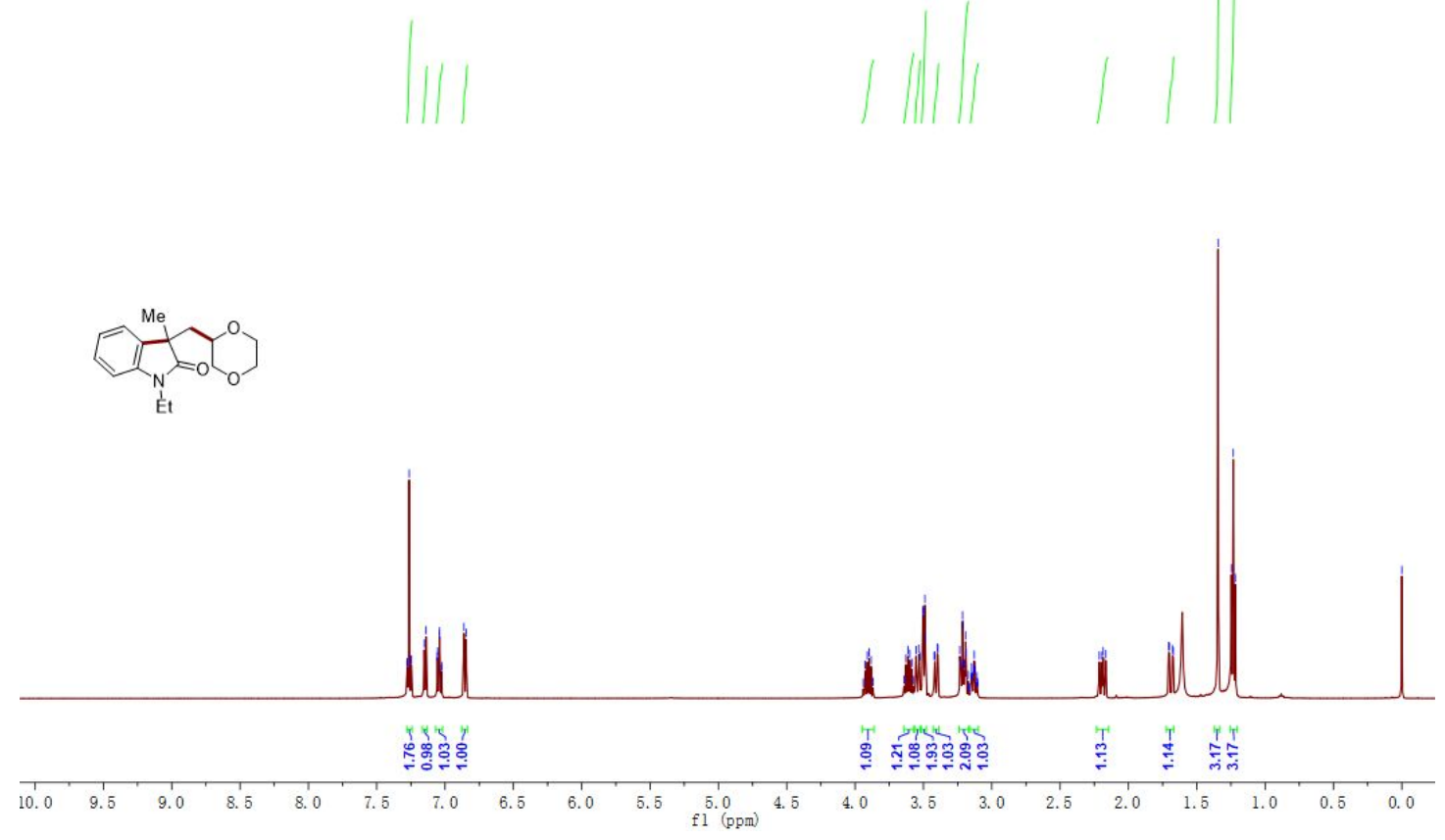

${ }^{13} \mathrm{C}\left\{{ }^{1} \mathrm{H}\right\}$ NMR of $\mathbf{3 n b} \mathbf{2}$ (125 MHz, $\left.\mathrm{CDCl}_{3}\right)$

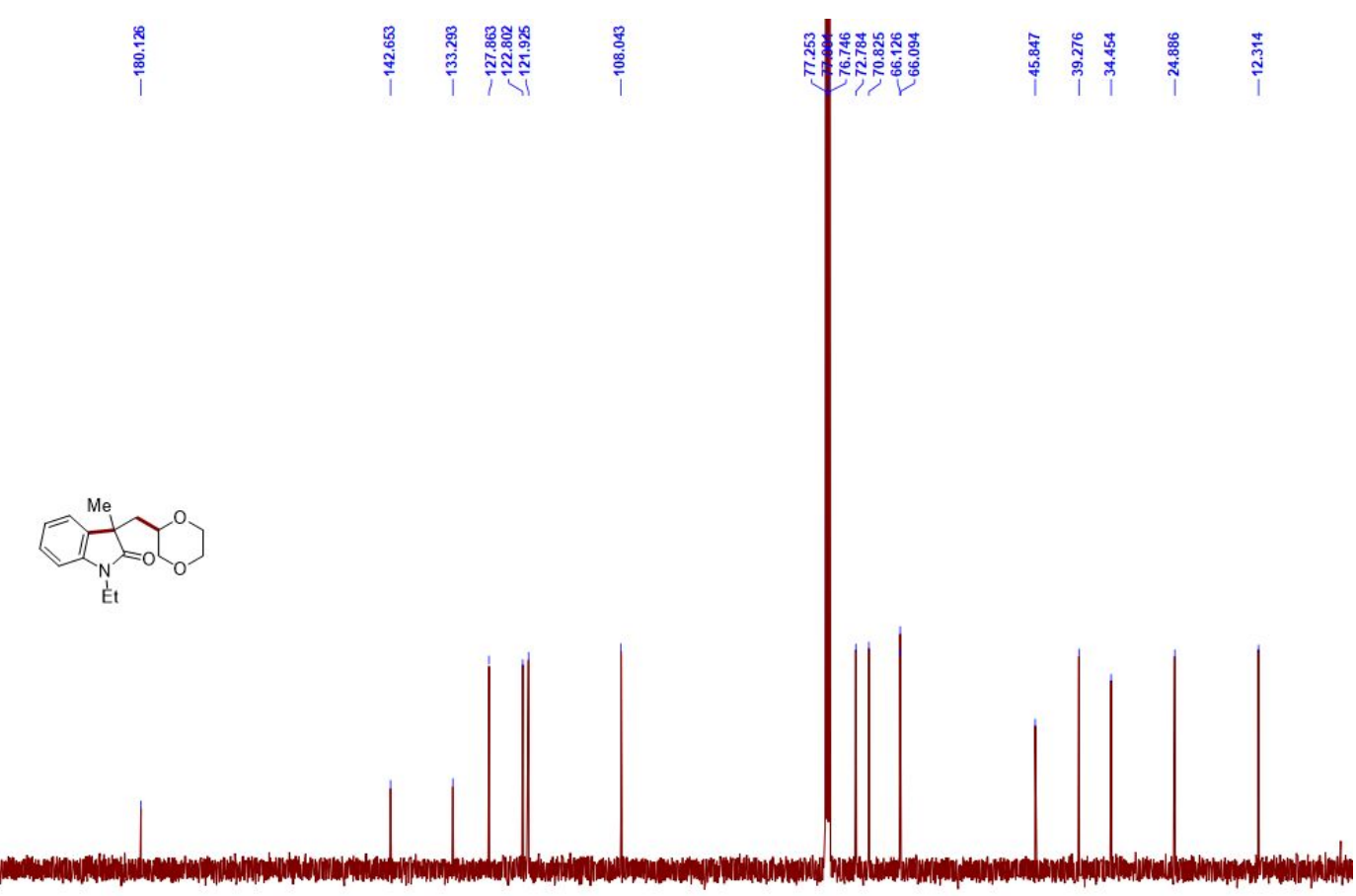

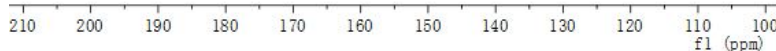


${ }^{1} \mathrm{H}$ NMR of 3ob-1 (500 MHz, $\left.\mathrm{CDCl}_{3}\right)$

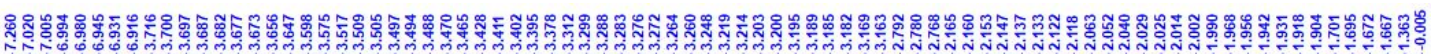

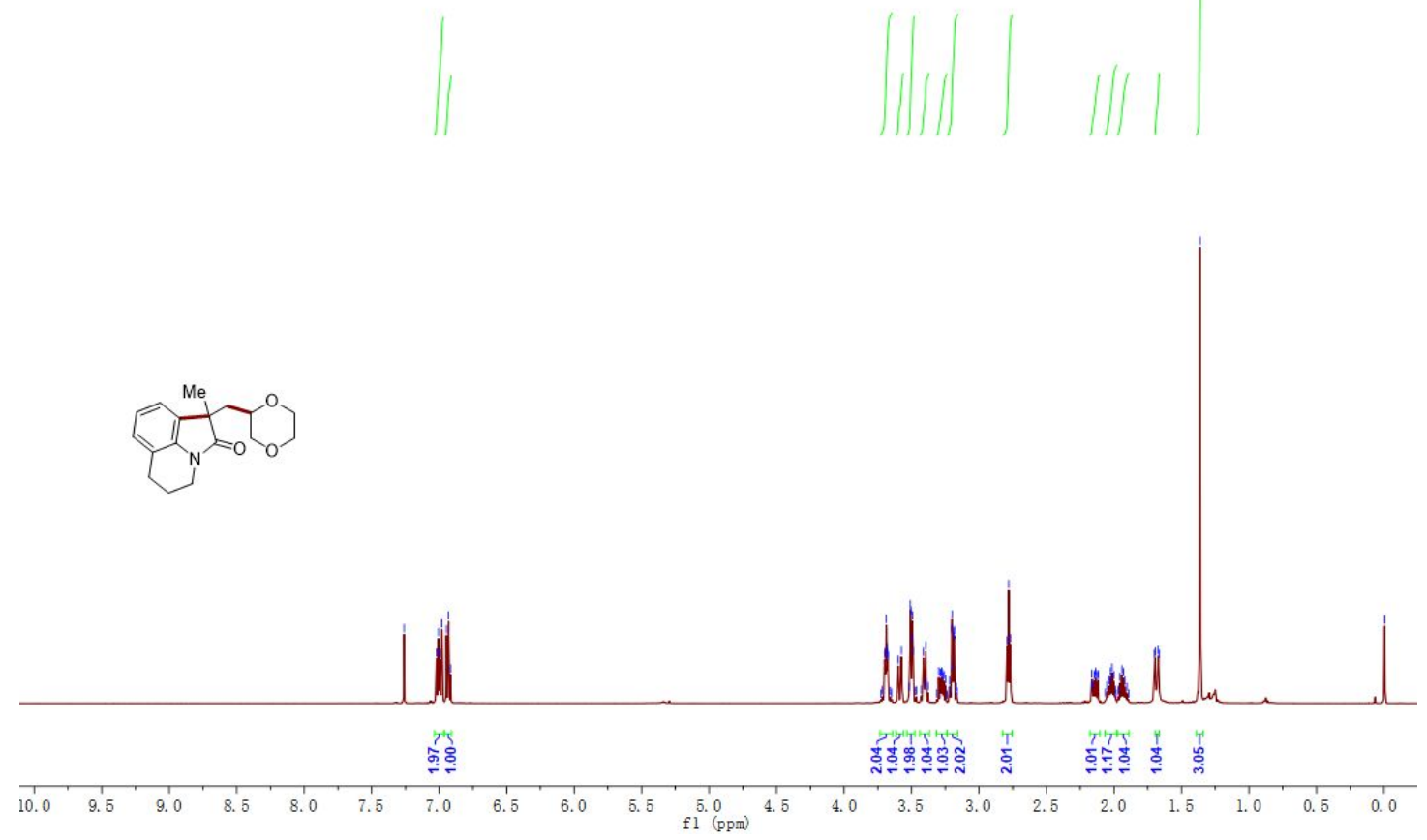

${ }^{13} \mathrm{C}\left\{{ }^{1} \mathrm{H}\right\}$ NMR of 3ob-1 (125 MHz, $\left.\mathrm{CDCl}_{3}\right)$

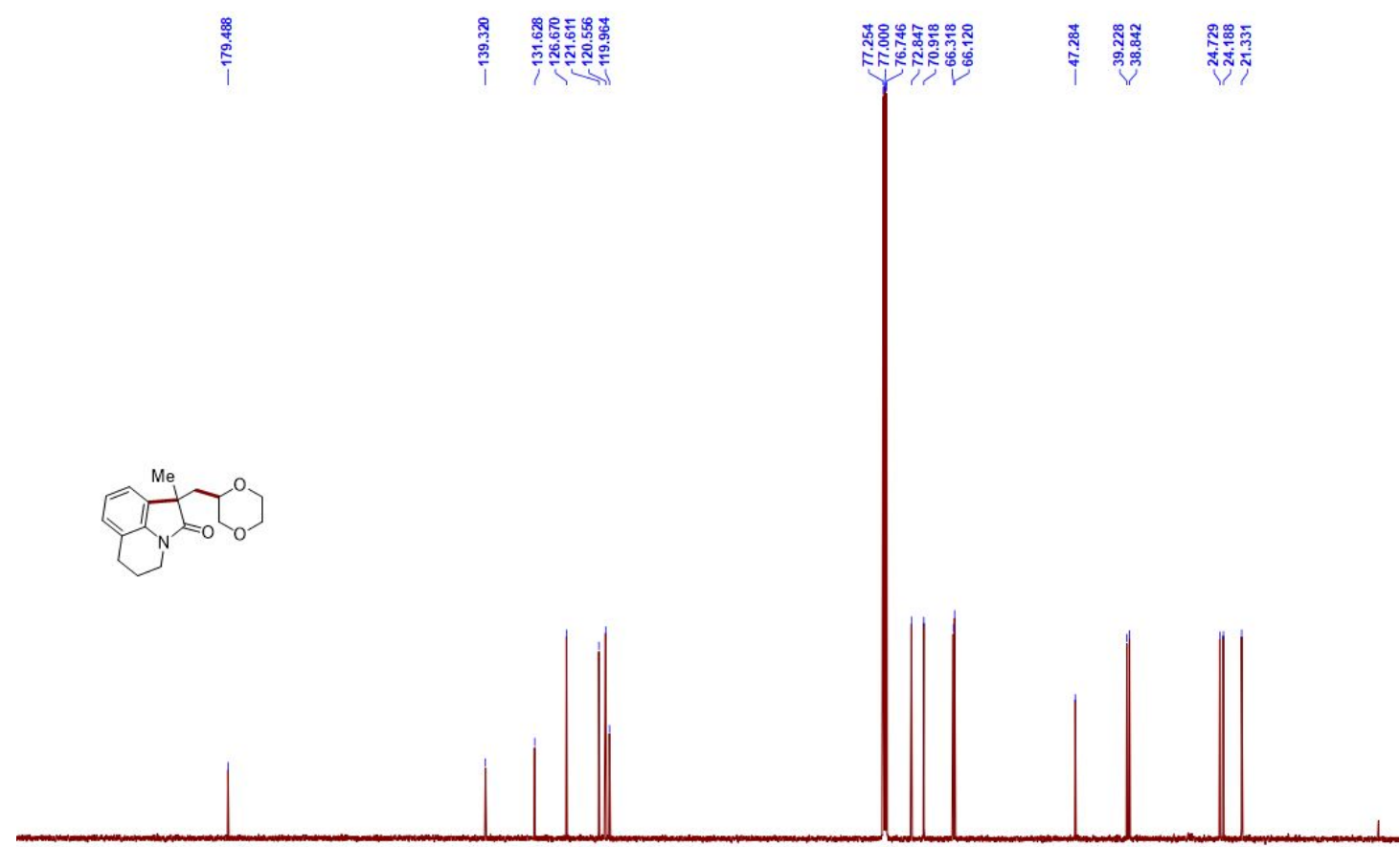

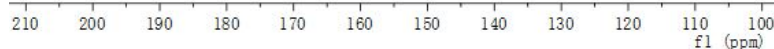


${ }^{1} \mathrm{H}$ NMR of 3ob-2 $\left(500 \mathrm{MHz}, \mathrm{CDCl}_{3}\right)$

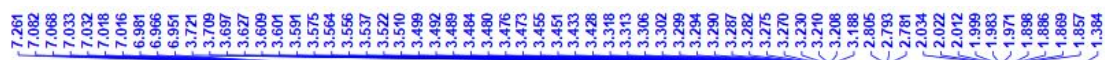

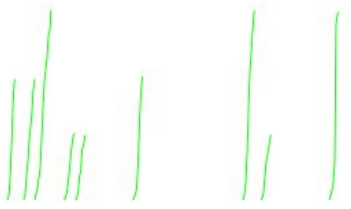

$\sum_{n=0}^{M e}$

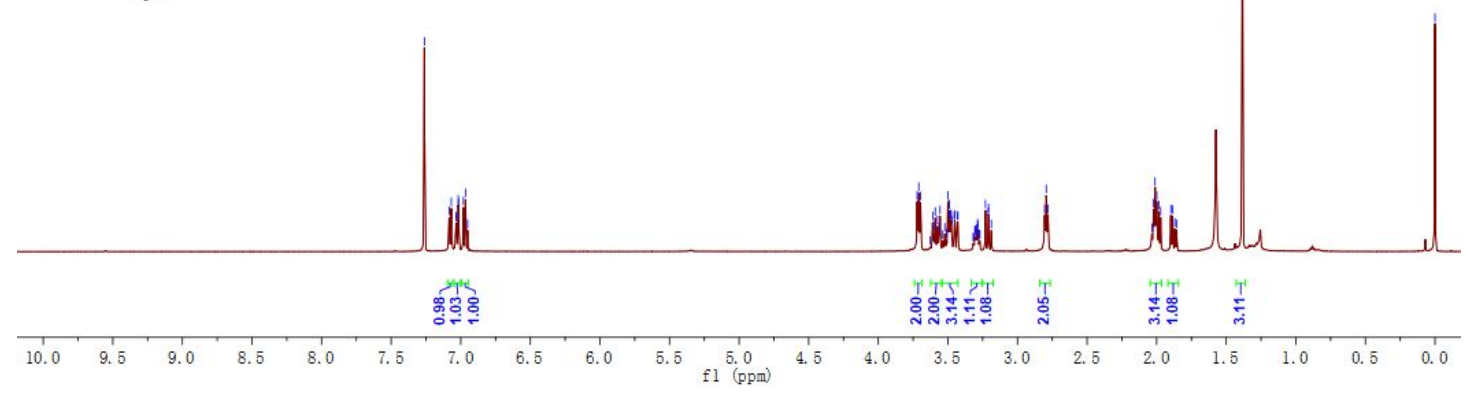

${ }^{13} \mathrm{C}\left\{{ }^{1} \mathrm{H}\right\}$ NMR of 3ob-2 $\left(125 \mathrm{MHz}, \mathrm{CDCl}_{3}\right)$

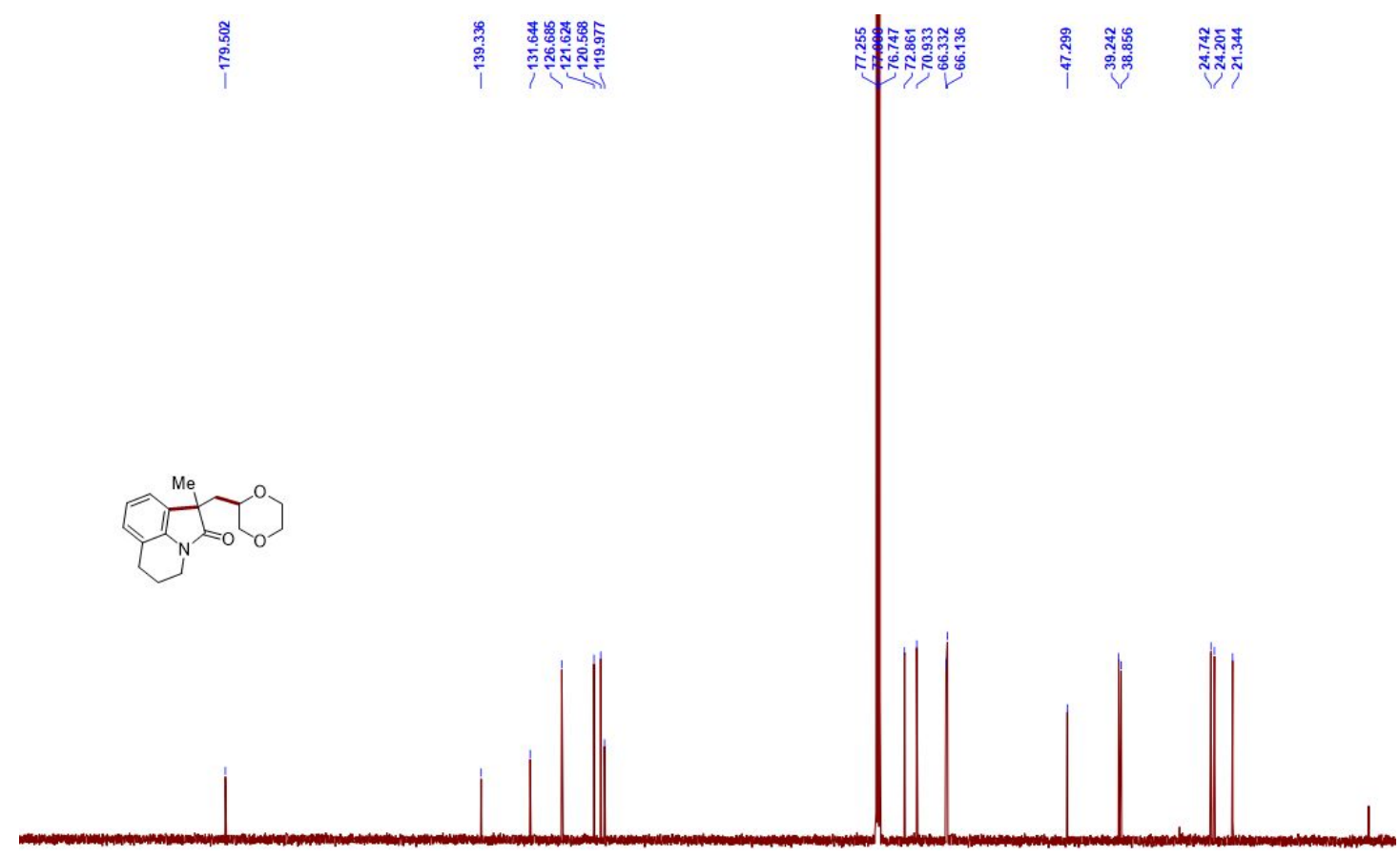

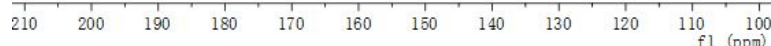


${ }^{1} \mathrm{H}$ NMR of 3pb-1 (500 MHz, $\left.\mathrm{CDCl}_{3}\right)$

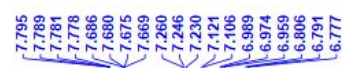

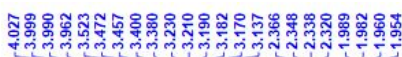
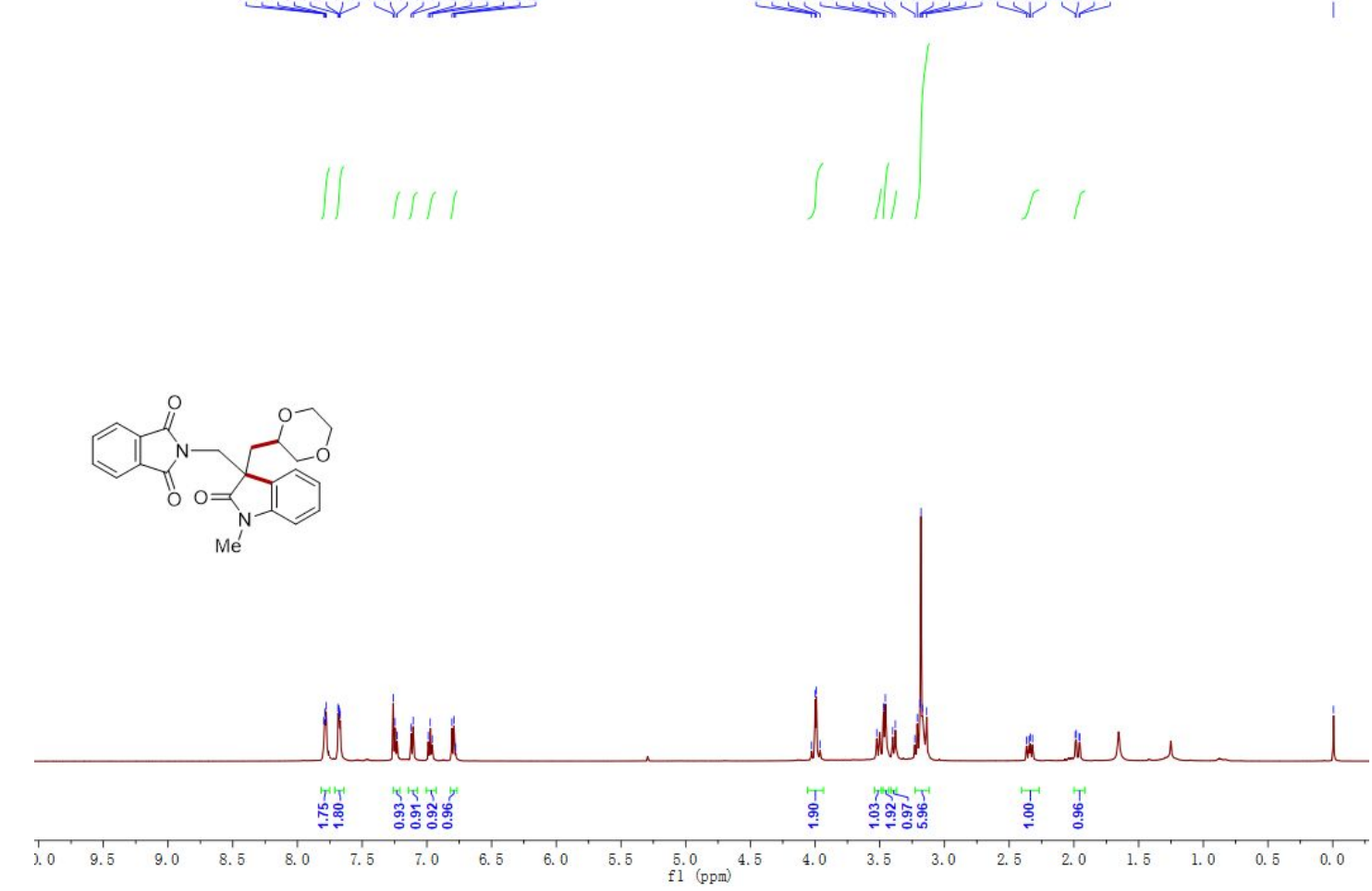

${ }^{13} \mathrm{C}\left\{{ }^{1} \mathrm{H}\right\}$ NMR of 3pb-1 (125 MHz, $\left.\mathrm{CDCl}_{3}\right)$

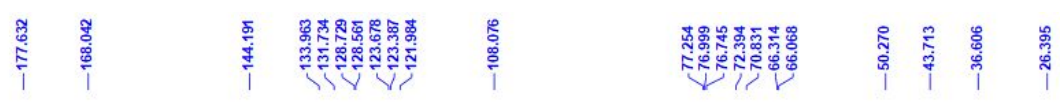
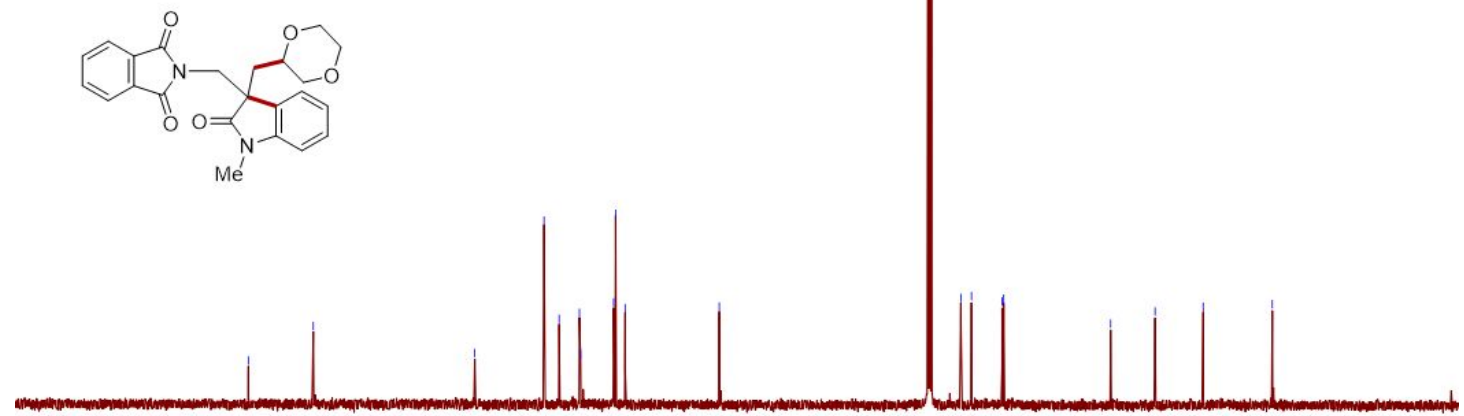

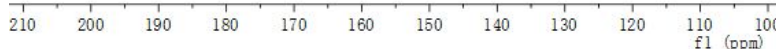


${ }^{1} \mathrm{H}$ NMR of 3pb-2 $\left(500 \mathrm{MHz}, \mathrm{CDCl}_{3}\right)$

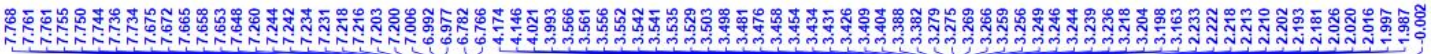
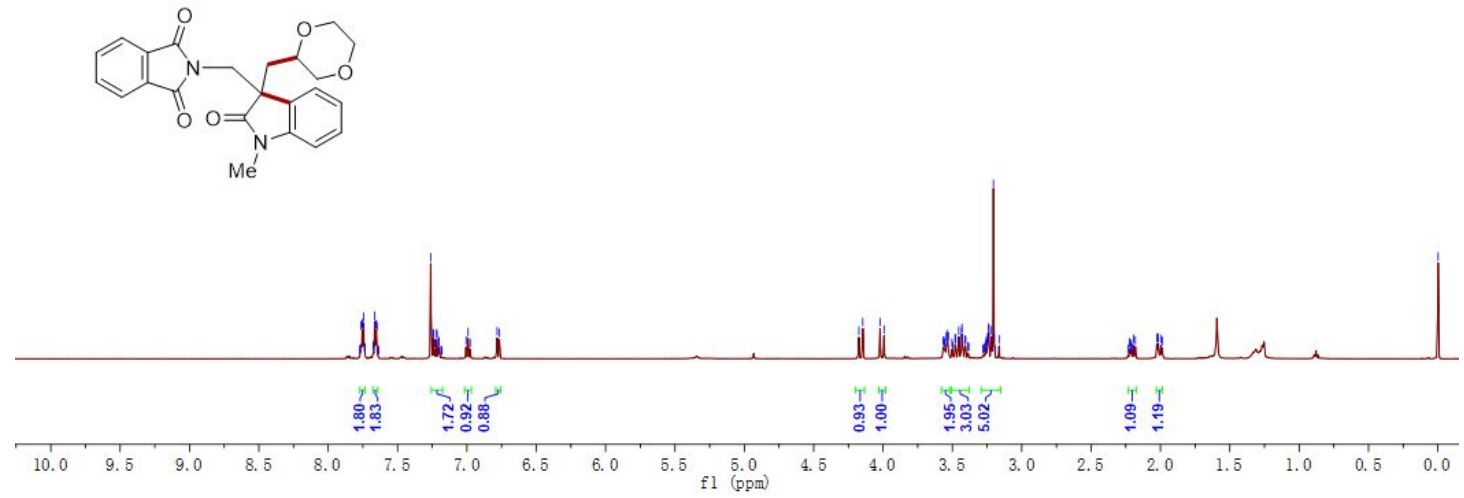

${ }^{13} \mathrm{C}\left\{{ }^{1} \mathrm{H}\right\}$ NMR of $\mathbf{3 p b}-2\left(125 \mathrm{MHz}, \mathrm{CDCl}_{3}\right)$

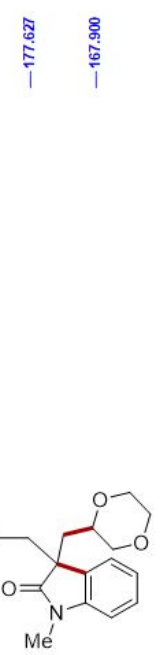

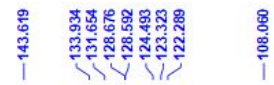

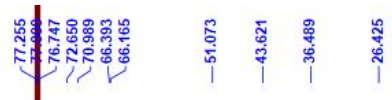
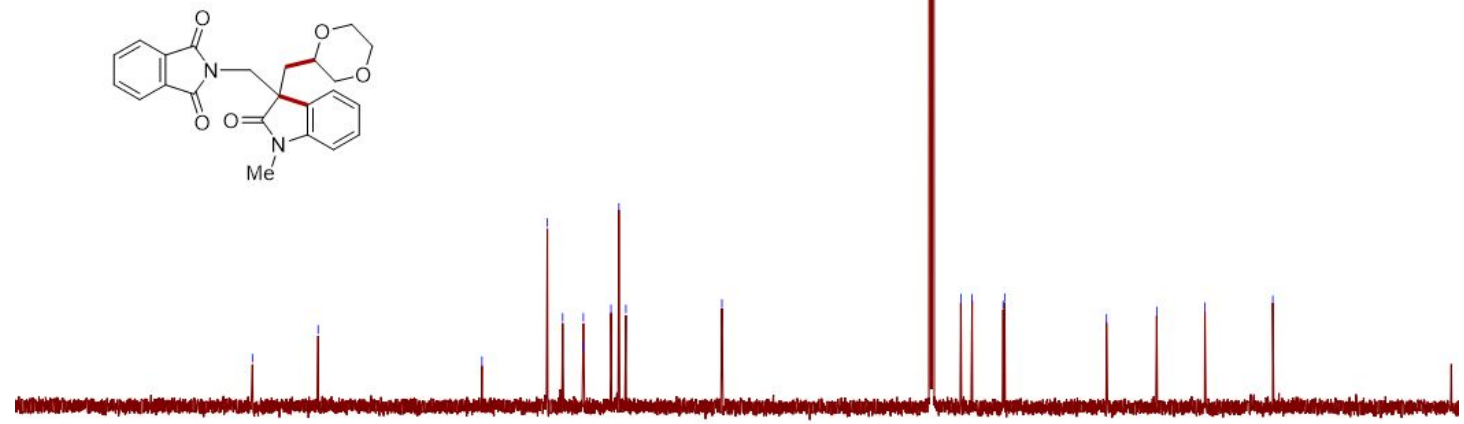

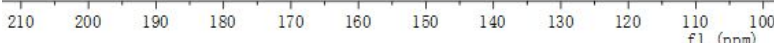


${ }^{1} \mathrm{H}$ NMR of 3qb-1 (600 MHz, $\left.\mathrm{CDCl}_{3}\right)$

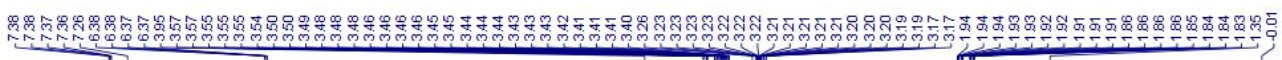

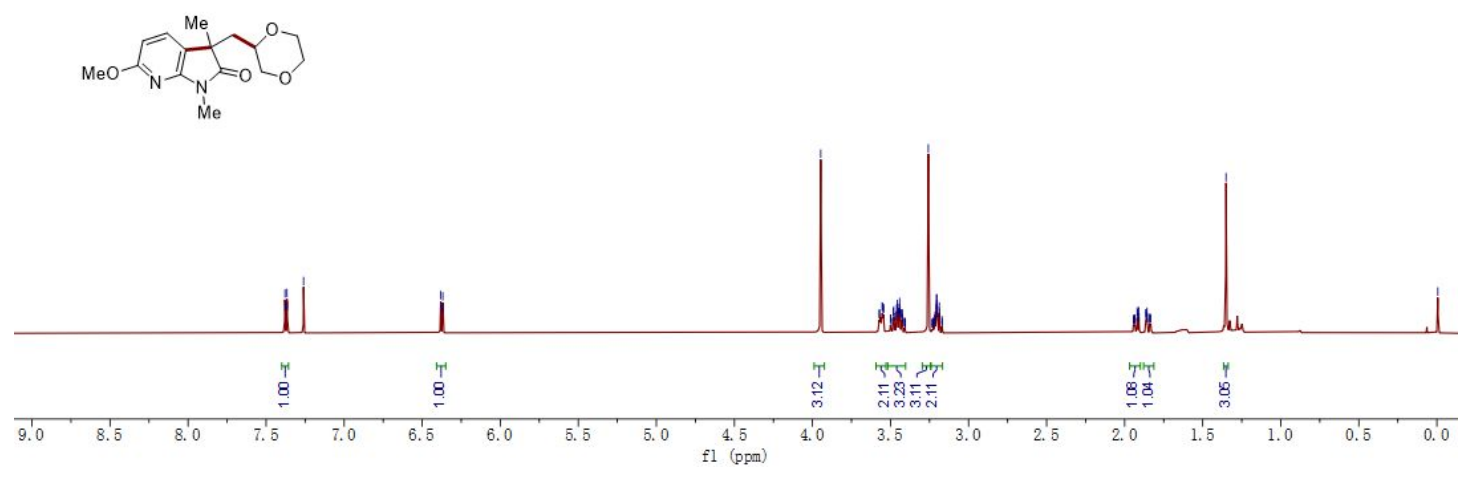

${ }^{13} \mathrm{C}\left\{{ }^{1} \mathrm{H}\right\}$ NMR of $\mathbf{3 q b}-\mathbf{1}\left(150 \mathrm{MHz}, \mathrm{CDCl}_{3}\right)$

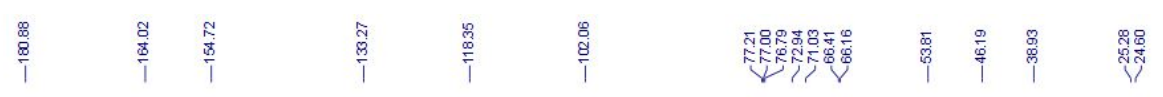

MeO

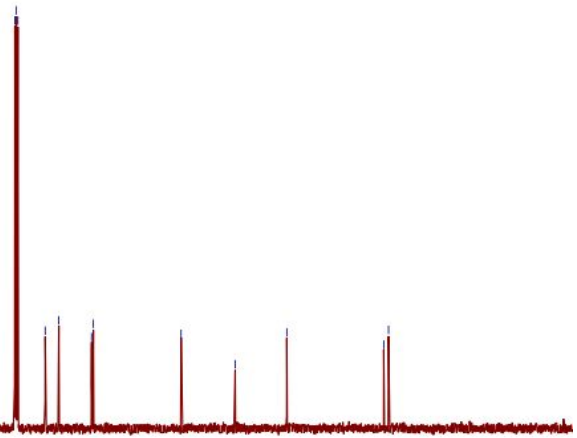

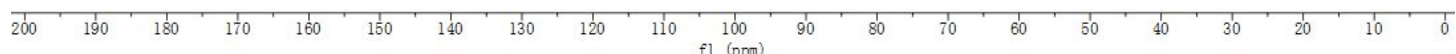


${ }^{1} \mathrm{H}$ NMR of 3qb-2 (600 MHz, $\left.\mathrm{CDCl}_{3}\right)$

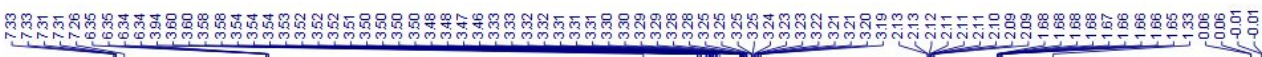
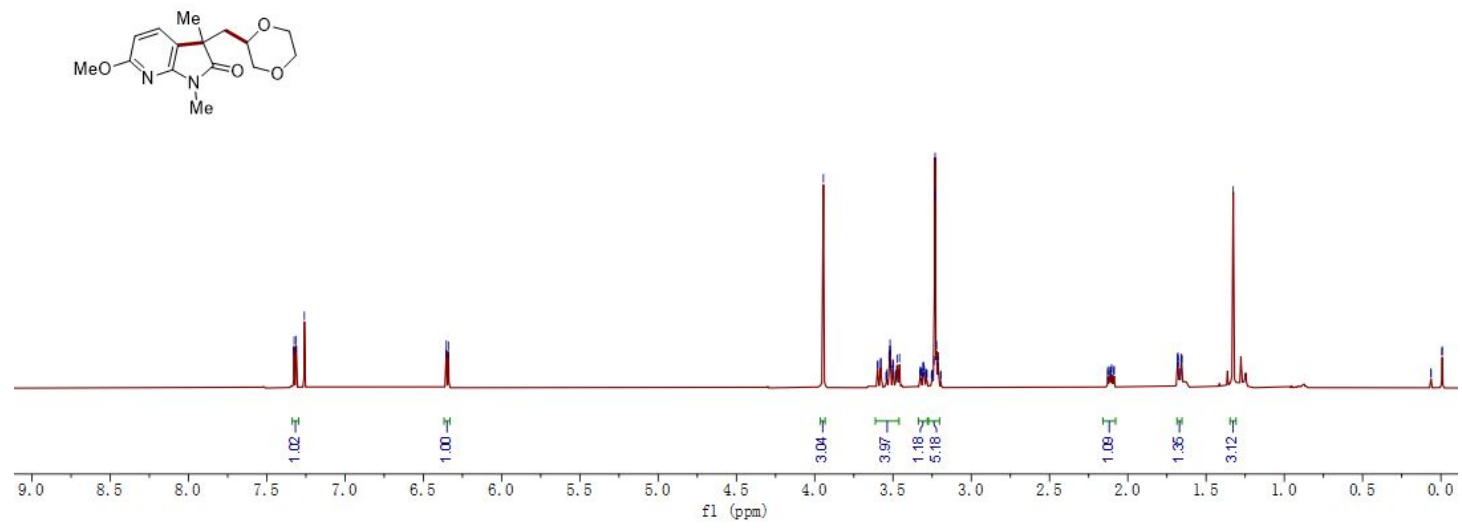

${ }^{13} \mathrm{C}\left\{{ }^{1} \mathrm{H}\right\}$ NMR of $\mathbf{3 q b}-2\left(150 \mathrm{MHz}, \mathrm{CDCl}_{3}\right)$

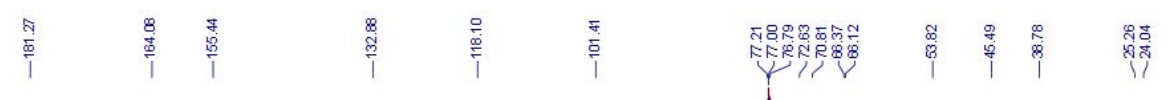
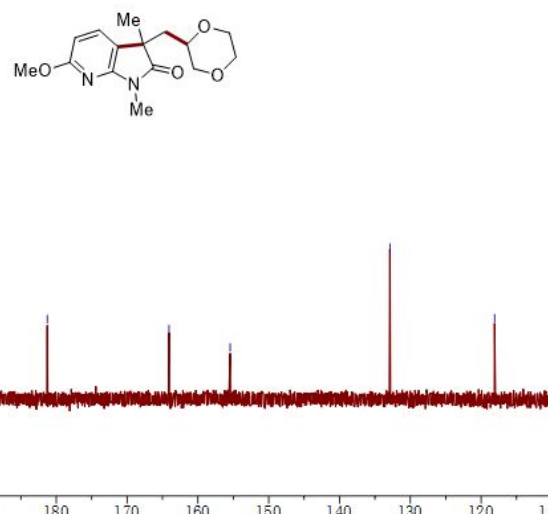

$\underset{f 1(p p g a)}{100}$ 
${ }^{1} \mathrm{H}$ NMR of 3 ac $\left(500 \mathrm{MHz}, \mathrm{CDCl}_{3}\right)$

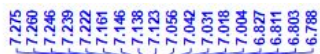

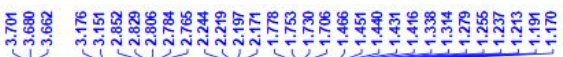

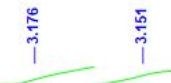

$\sum_{\substack{N \\ \text { Me }}}^{M e}=0$
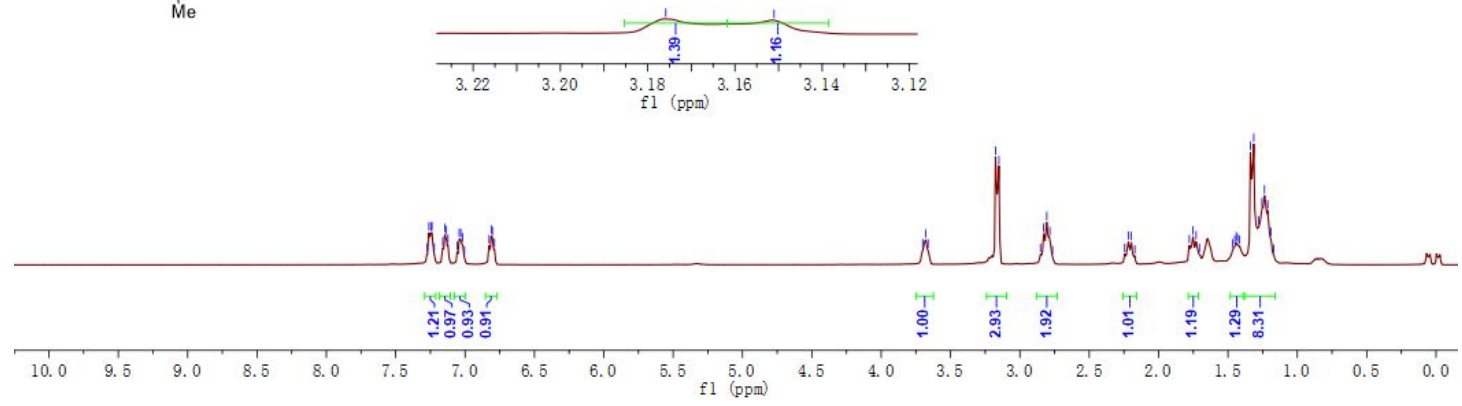

${ }^{13} \mathrm{C}\left\{{ }^{1} \mathrm{H}\right\}$ NMR of $\mathbf{3 a c}\left(125 \mathrm{MHz}, \mathrm{CDCl}_{3}\right)$

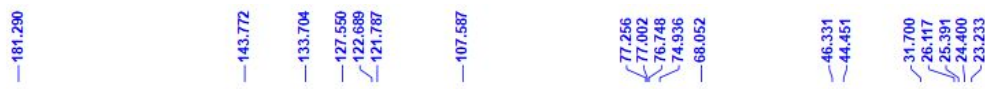
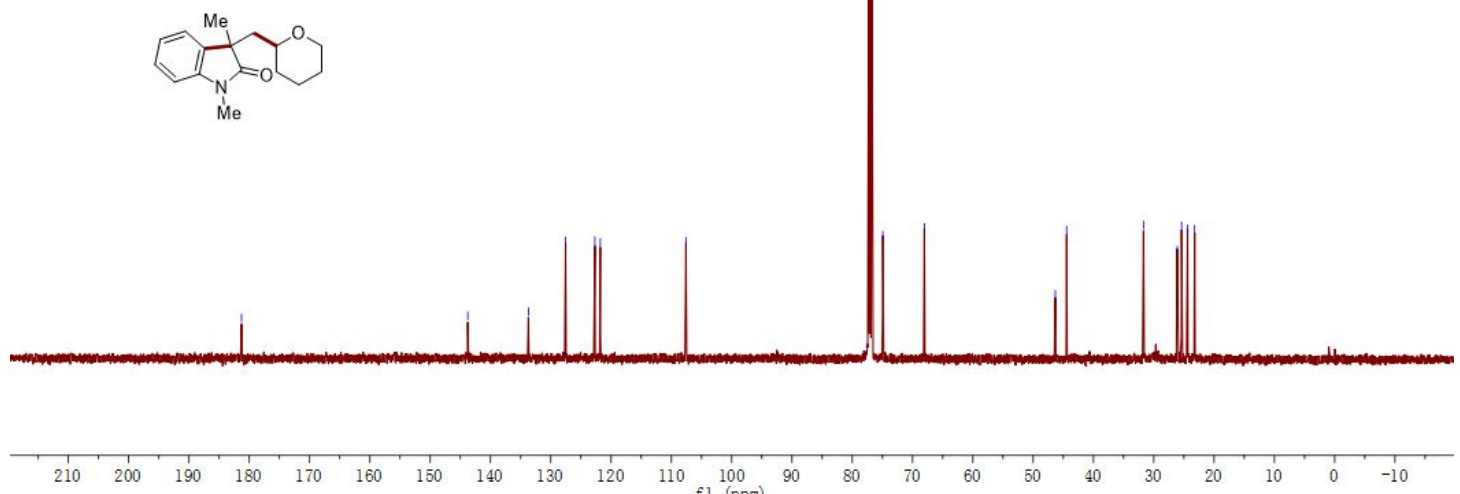
${ }^{1} \mathrm{H}$ NMR of 3 ad $\left(500 \mathrm{MHz}, \mathrm{CDCl}_{3}\right)$

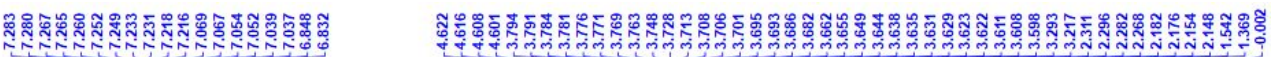

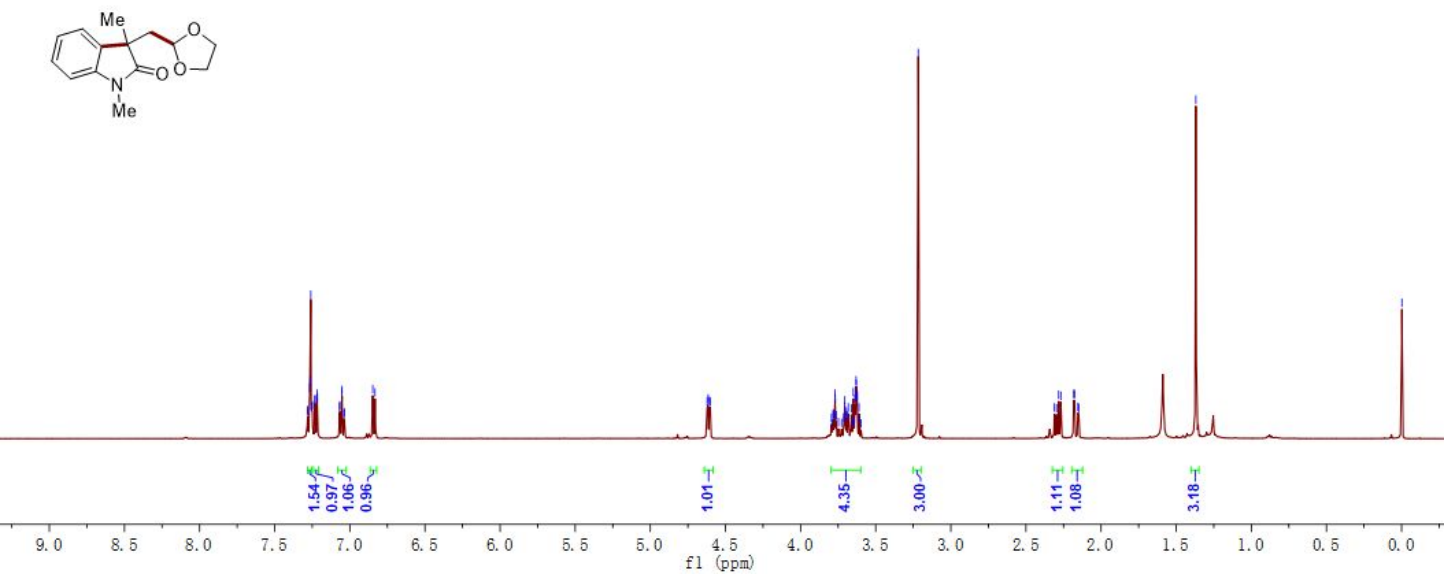

${ }^{13} \mathrm{C}\left\{{ }^{1} \mathrm{H}\right\} \mathrm{NMR}$ of $\mathbf{3 a d}\left(125 \mathrm{MHz}, \mathrm{CDCl}_{3}\right)$

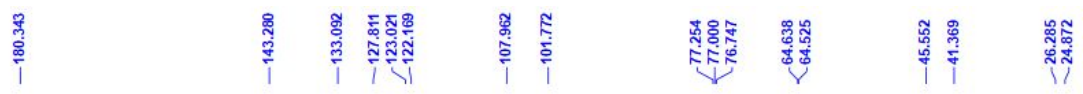
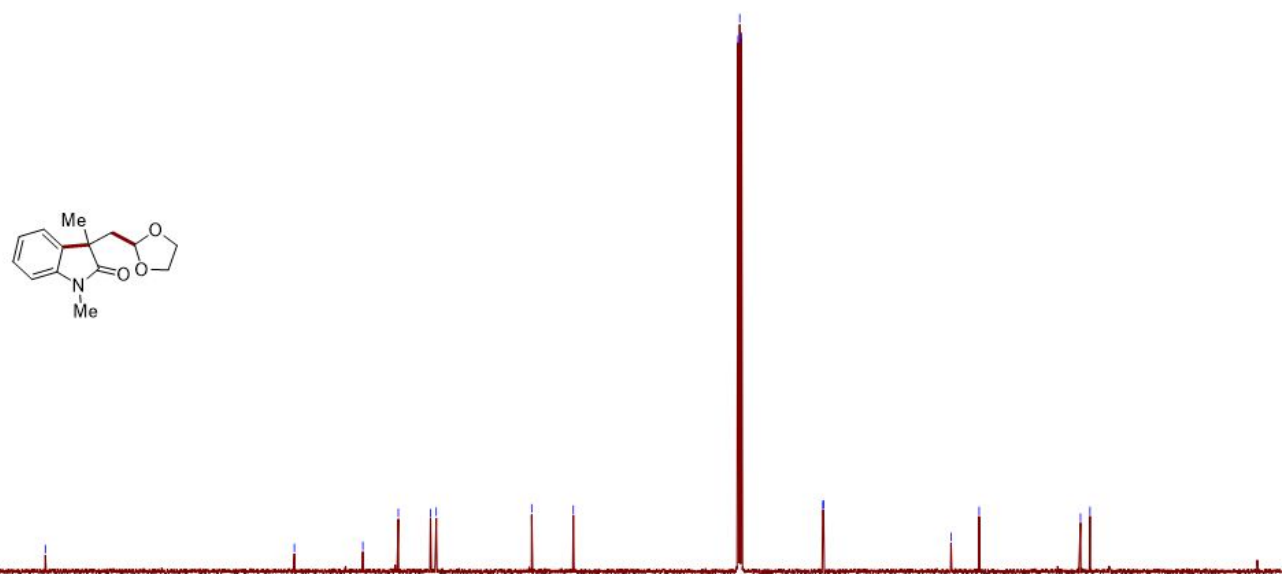

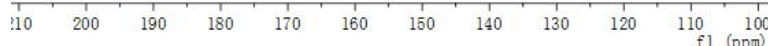


${ }^{1} \mathrm{H}$ NMR of 3 ae $\left(500 \mathrm{MHz}, \mathrm{CDCl}_{3}\right)$

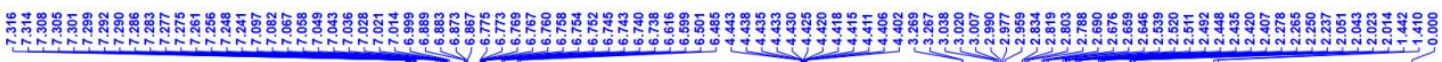
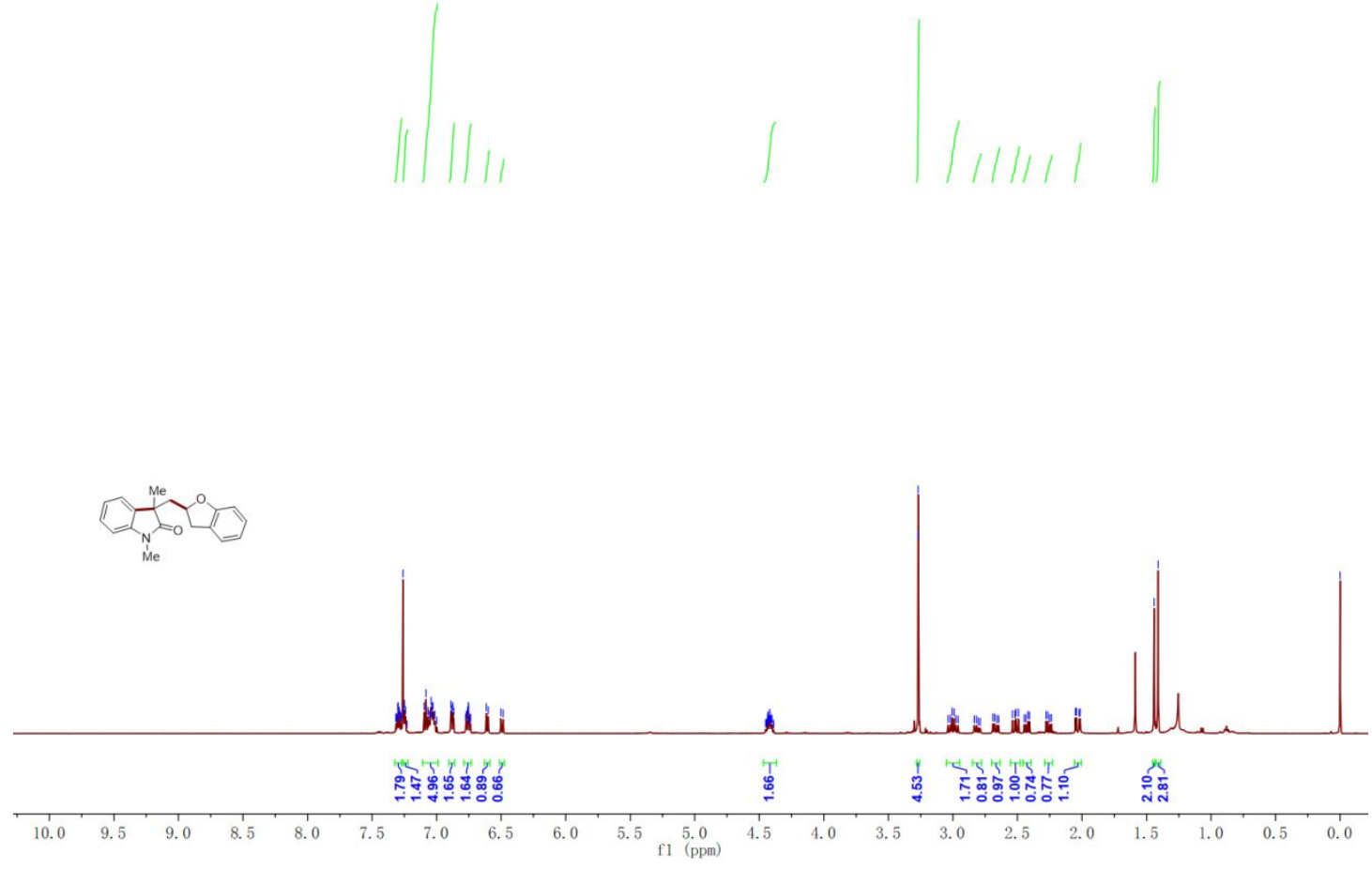

${ }^{13} \mathrm{C}\left\{{ }^{1} \mathrm{H}\right\}$ NMR of $\mathbf{3 a e}\left(125 \mathrm{MHz}, \mathrm{CDCl}_{3}\right)$

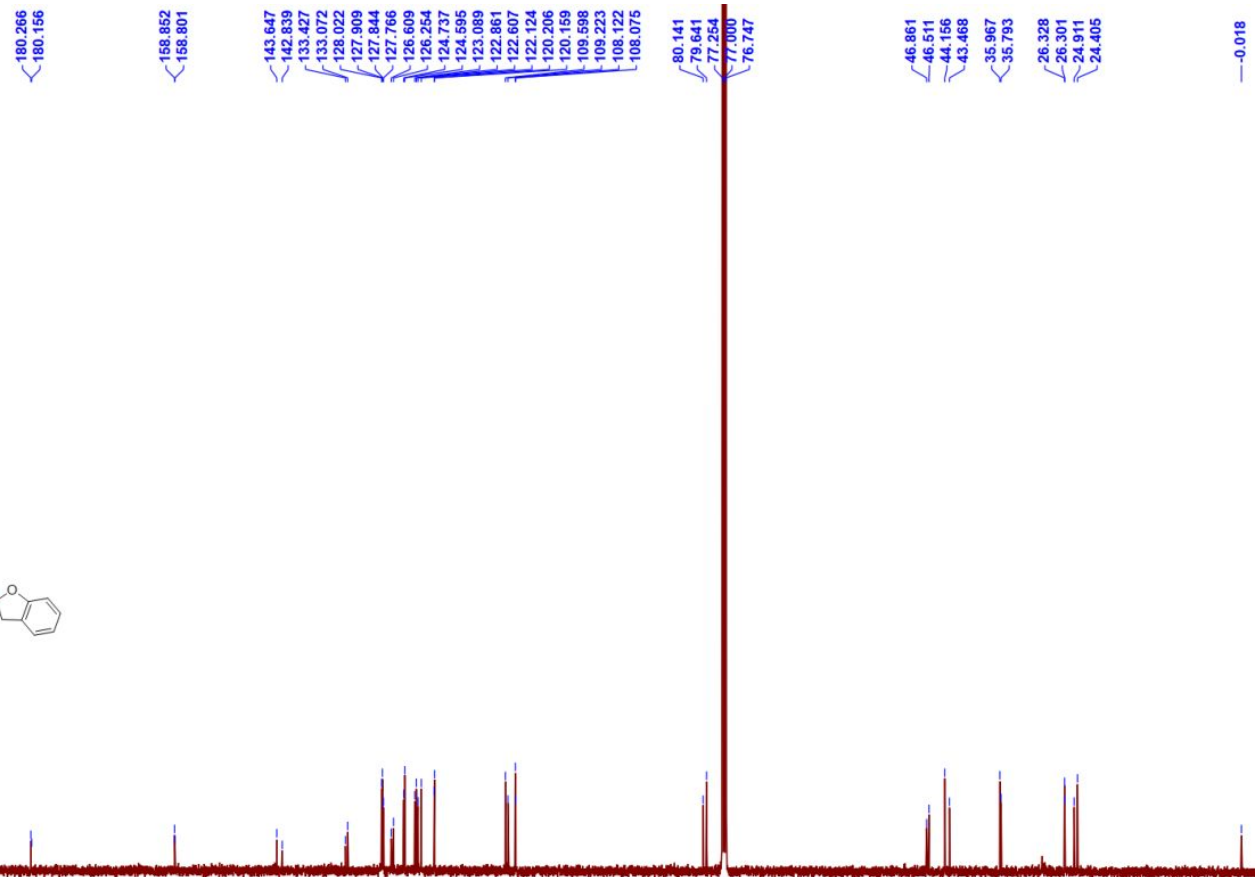

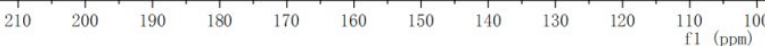


${ }^{1} \mathrm{H} \mathrm{NMR}$ of 3ae' $\left(500 \mathrm{MHz}, \mathrm{CDCl}_{3}\right)$

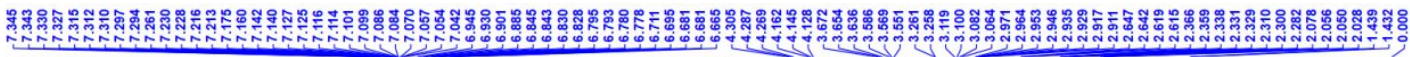

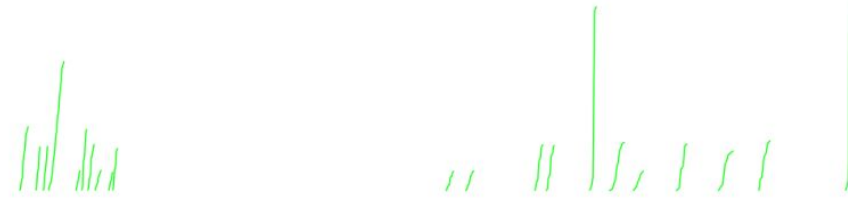

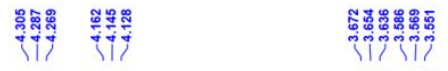
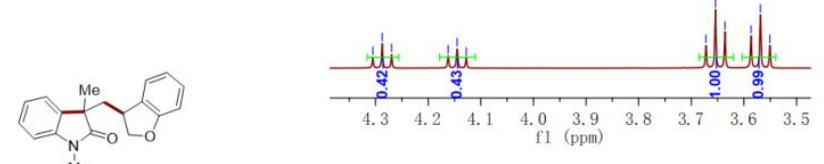

Me

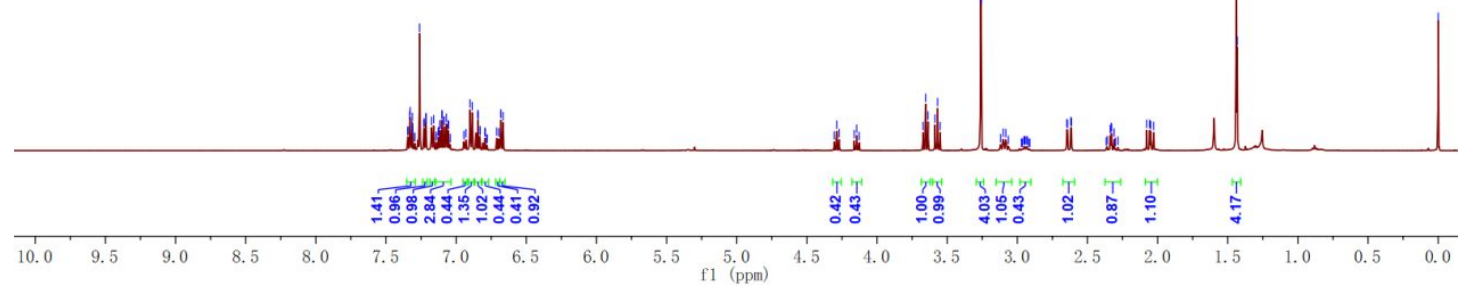

${ }^{13} \mathrm{C}\left\{{ }^{1} \mathrm{H}\right\}$ NMR of 3ae' $\left(125 \mathrm{MHz}, \mathrm{CDCl}_{3}\right)$
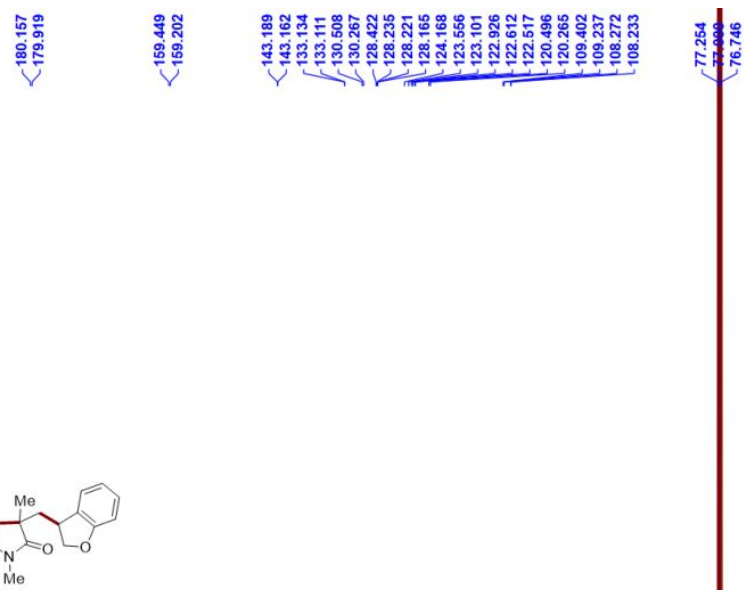

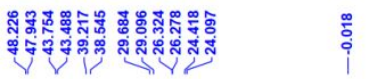

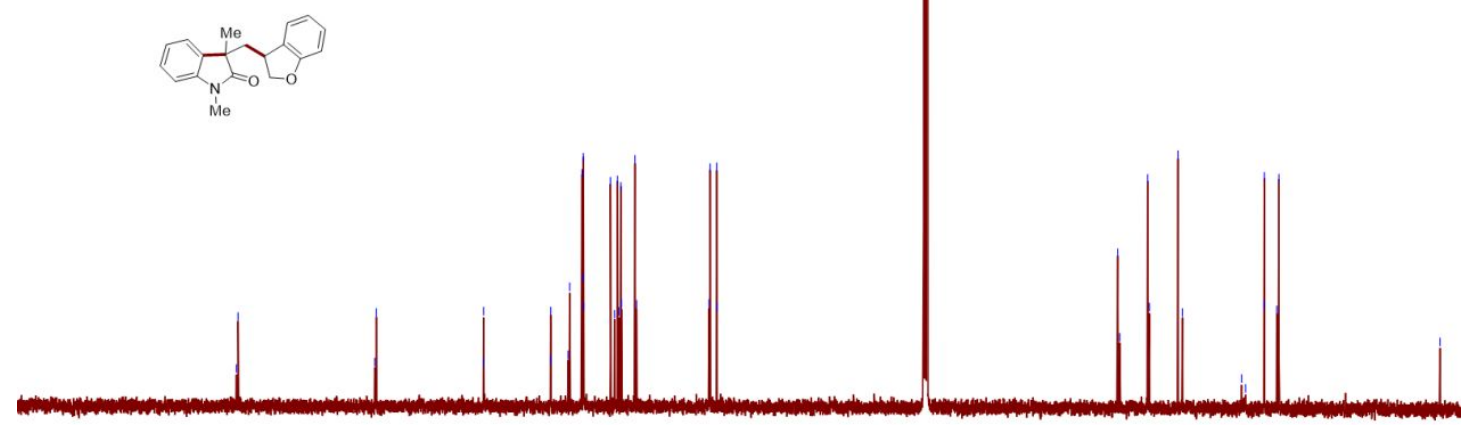

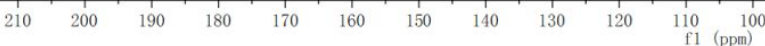


${ }^{1} \mathrm{H}$ NMR of 3 af $\left(500 \mathrm{MHz}, \mathrm{CDCl}_{3}\right)$

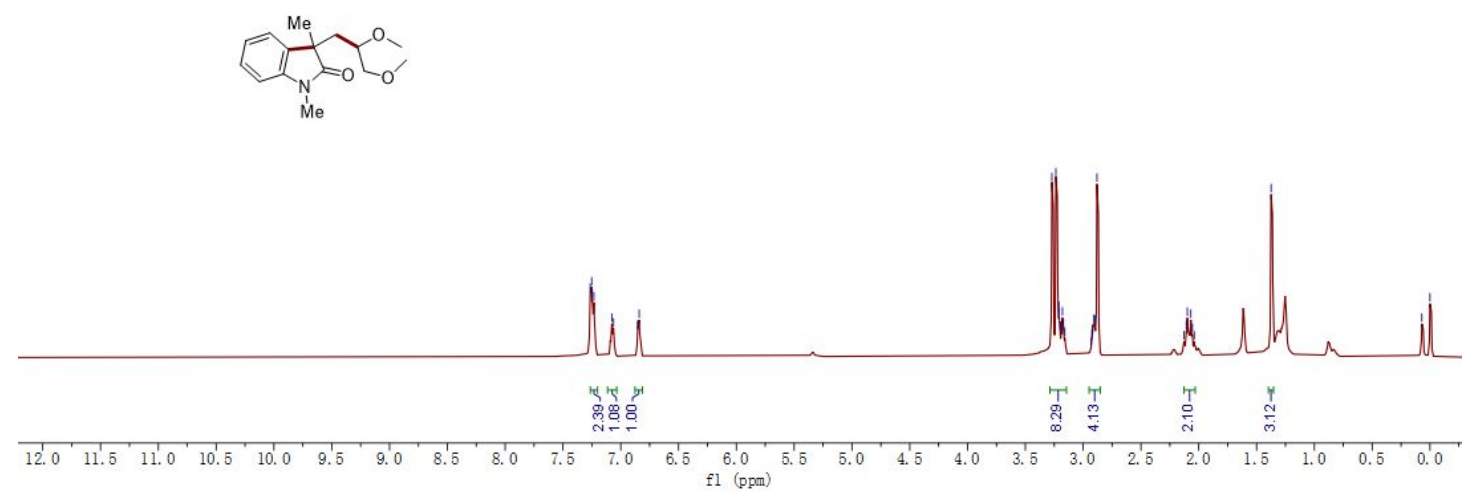

${ }^{13} \mathrm{C}\left\{{ }^{1} \mathrm{H}\right\}$ NMR of 3 af $\left(125 \mathrm{MHz}, \mathrm{CDCl}_{3}\right)$

(1)

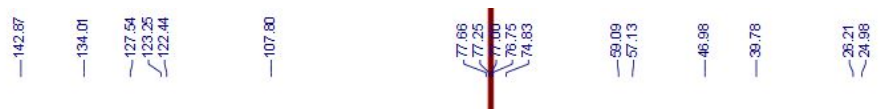

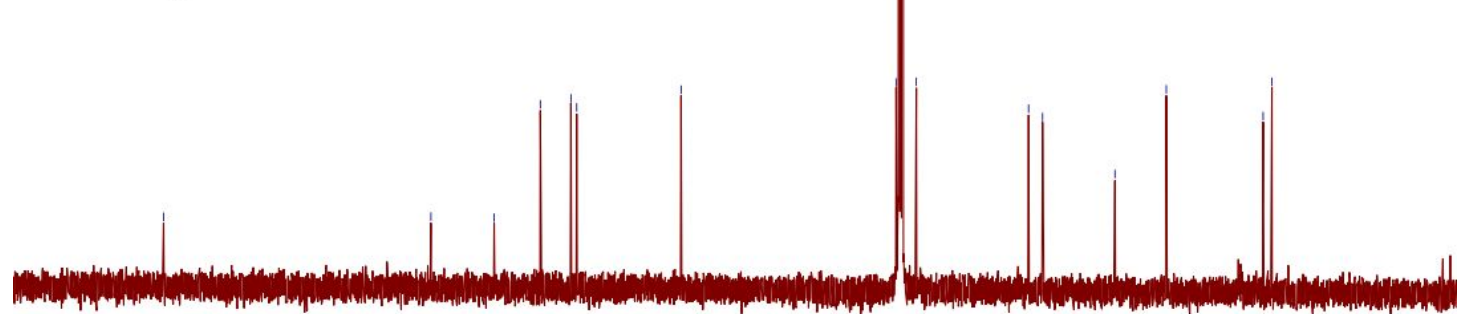

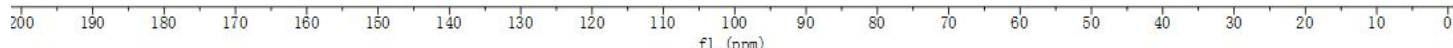


${ }^{1} \mathrm{H} \mathrm{NMR}$ of $3 \mathrm{ag}\left(500 \mathrm{MHz}, \mathrm{CDCl}_{3}\right)$

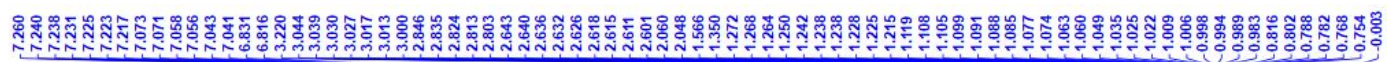

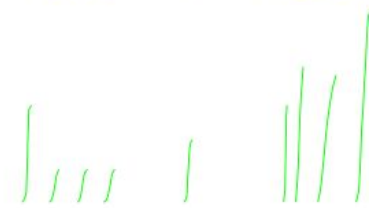

$\sum_{\substack{n \\ N=0}}^{M e}$

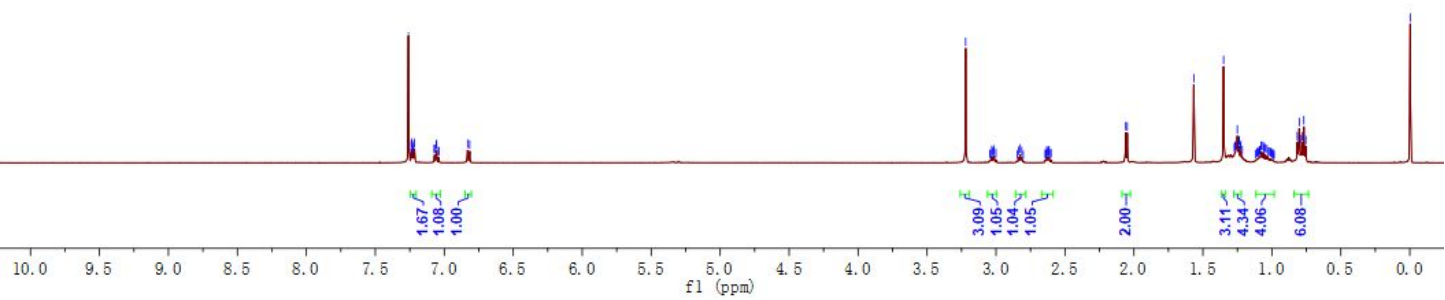

${ }^{13} \mathrm{C}\left\{{ }^{1} \mathrm{H}\right\}$ NMR of $\mathbf{3 a g}\left(125 \mathrm{MHz}, \mathrm{CDCl}_{3}\right)$

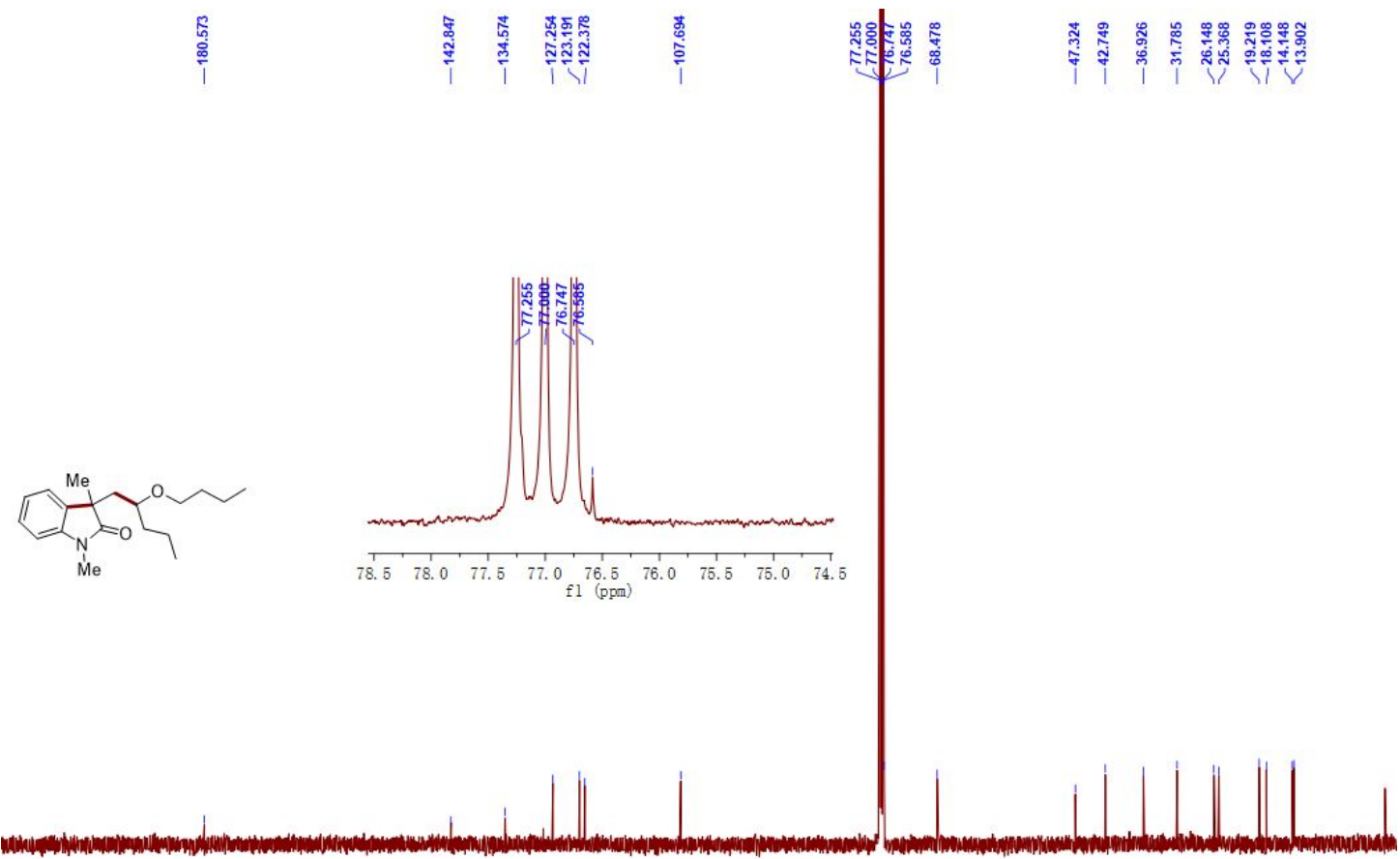

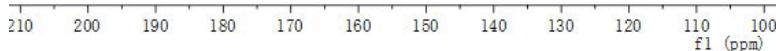


${ }^{1} \mathrm{H}$ NMR of $3 a i$ (500 MHz, $\left.\mathrm{CDCl}_{3}\right)$

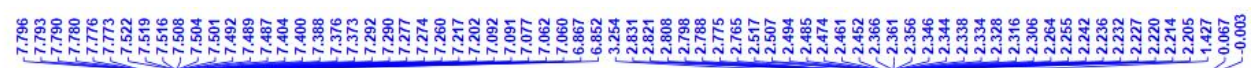

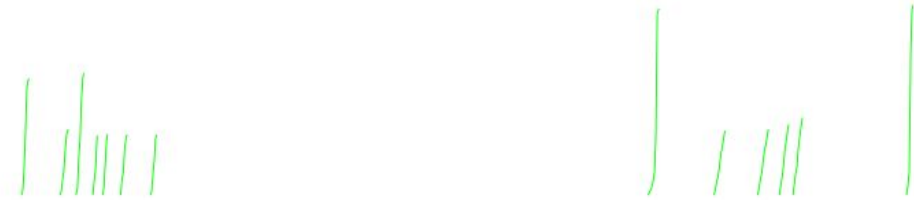

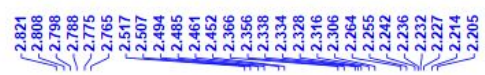
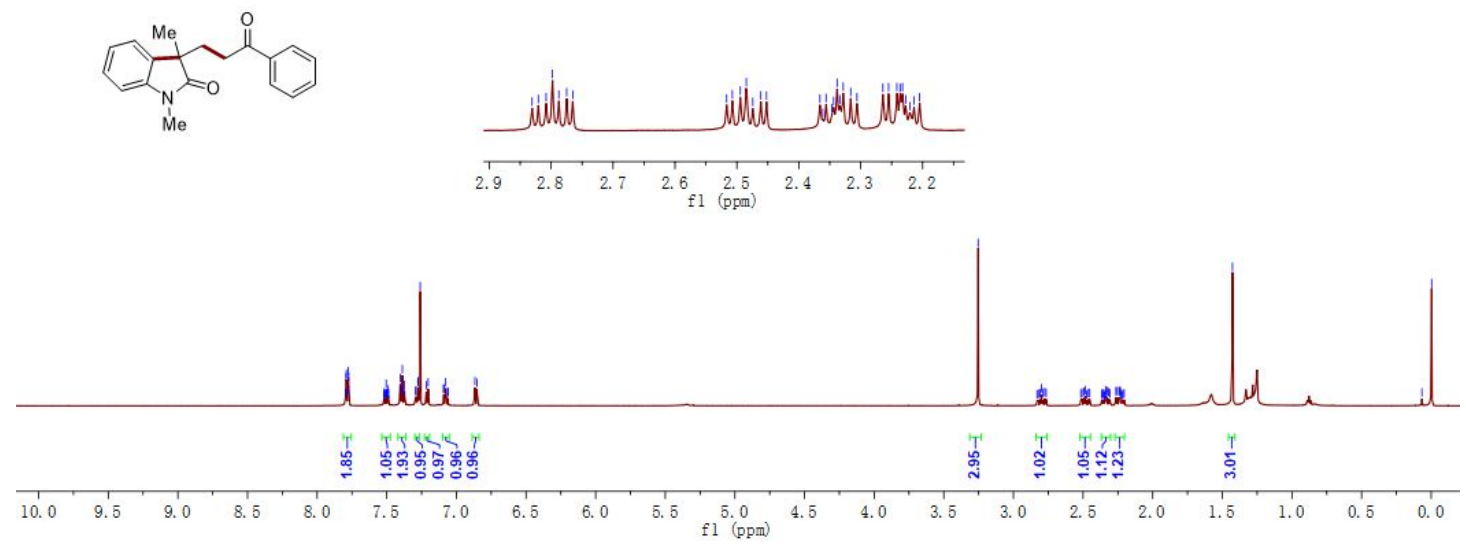

${ }^{13} \mathrm{C}\left\{{ }^{1} \mathrm{H}\right\}$ NMR of 3ai $\left(125 \mathrm{MHz}, \mathrm{CDCl}_{3}\right)$

\begin{tabular}{|c|c|c|c|c|c|c|}
\hline ब్ల్ల & $\stackrel{8}{\circ}$ & 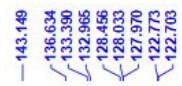 & 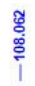 & 䒿骂 & 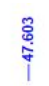 & 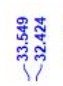 \\
\hline
\end{tabular}
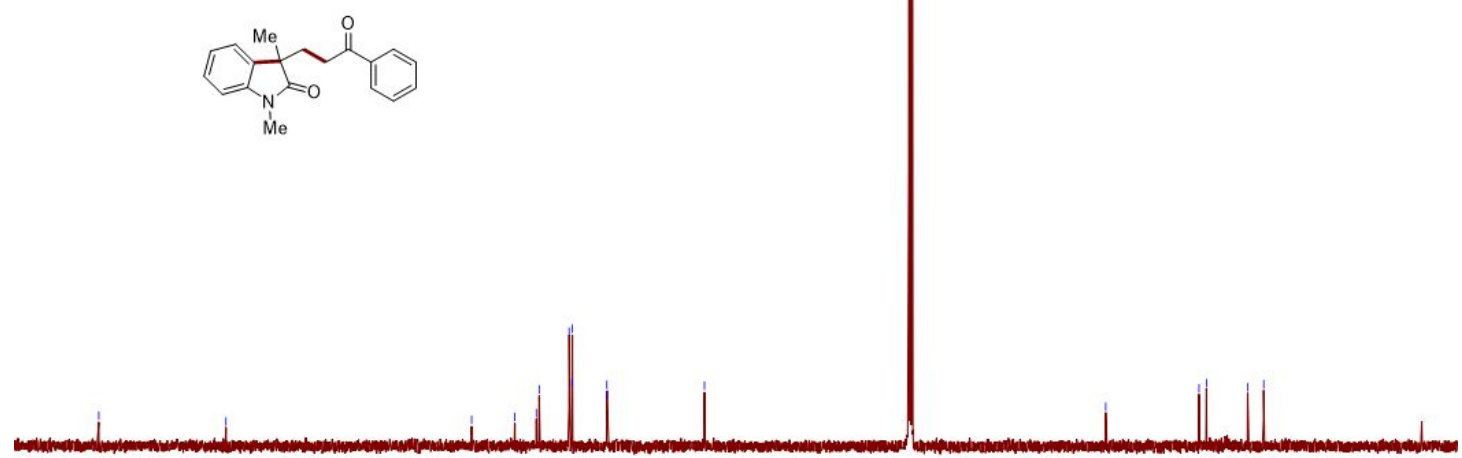

$\begin{array}{llllllllllll}210 & 200 & 190 & 180 & 170 & 160 & 150 & 140 & 130 & 120 & 110 & 100\end{array}$ 
${ }^{1} \mathrm{H}$ NMR of 3aj $\left(500 \mathrm{MHz}, \mathrm{CDCl}_{3}\right)$

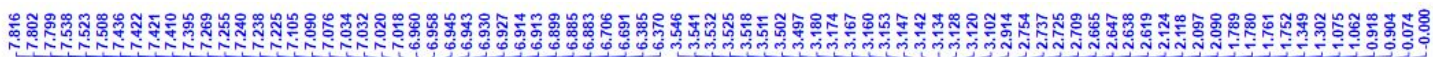
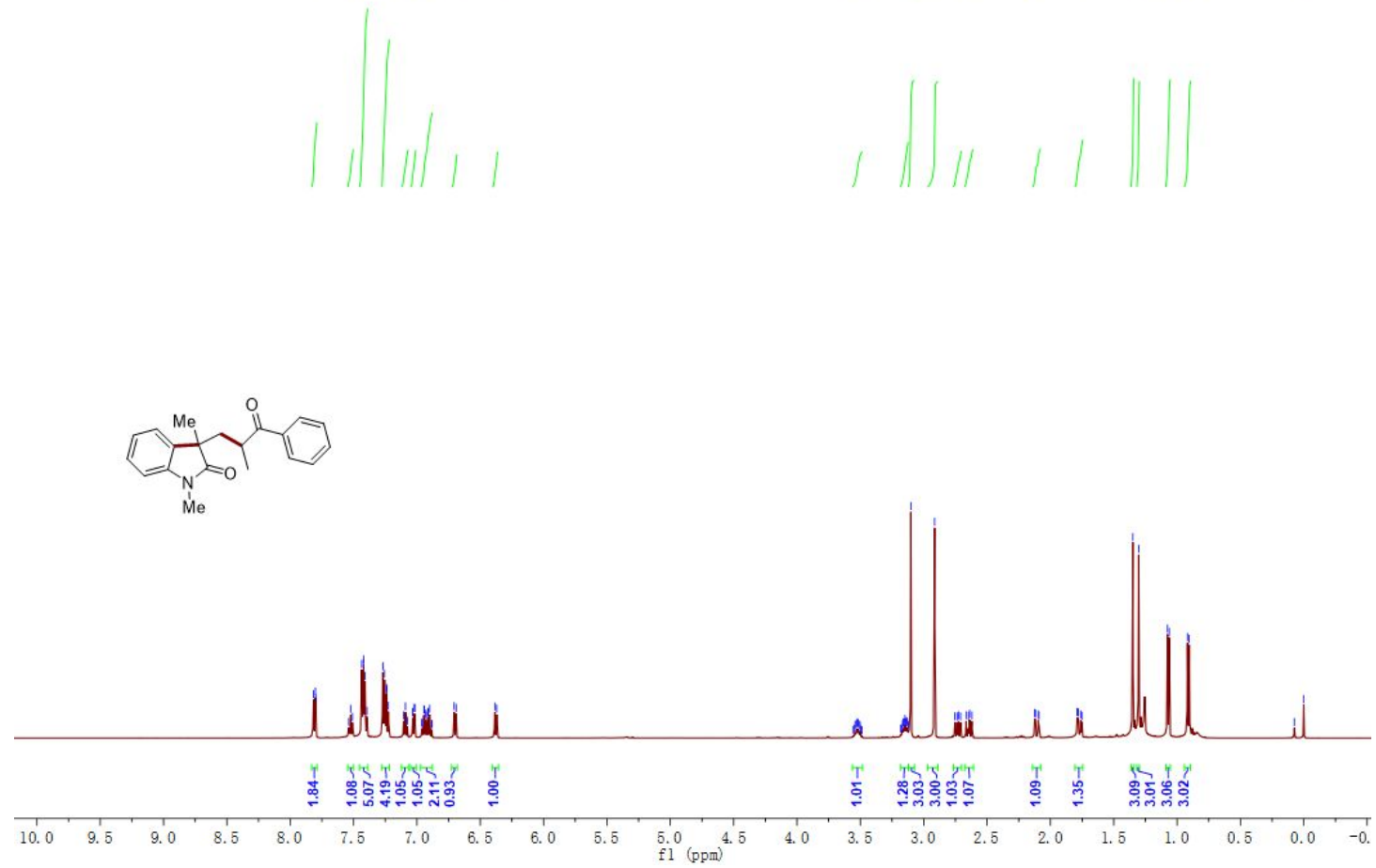

${ }^{13} \mathrm{C}\left\{{ }^{1} \mathrm{H}\right\}$ NMR of $\mathbf{3 a j}\left(125 \mathrm{MHz}, \mathrm{CDCl}_{3}\right)$

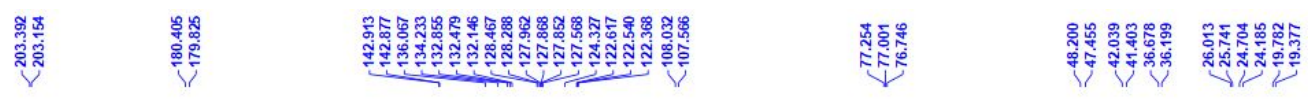
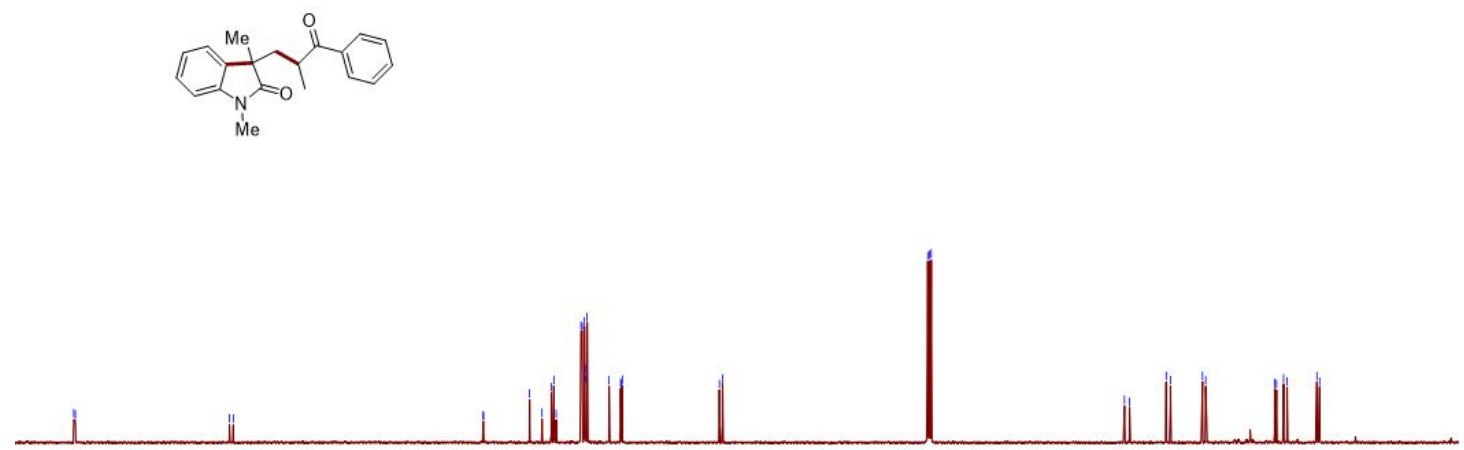

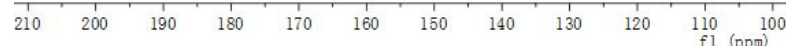


${ }^{1} \mathrm{H}$ NMR of 3ak $\left(500 \mathrm{MHz}, \mathrm{CDCl}_{3}\right)$

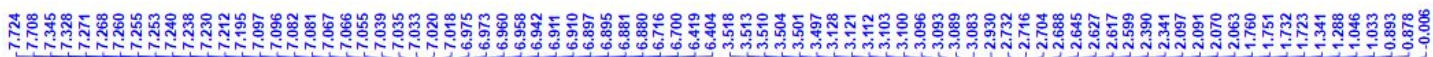
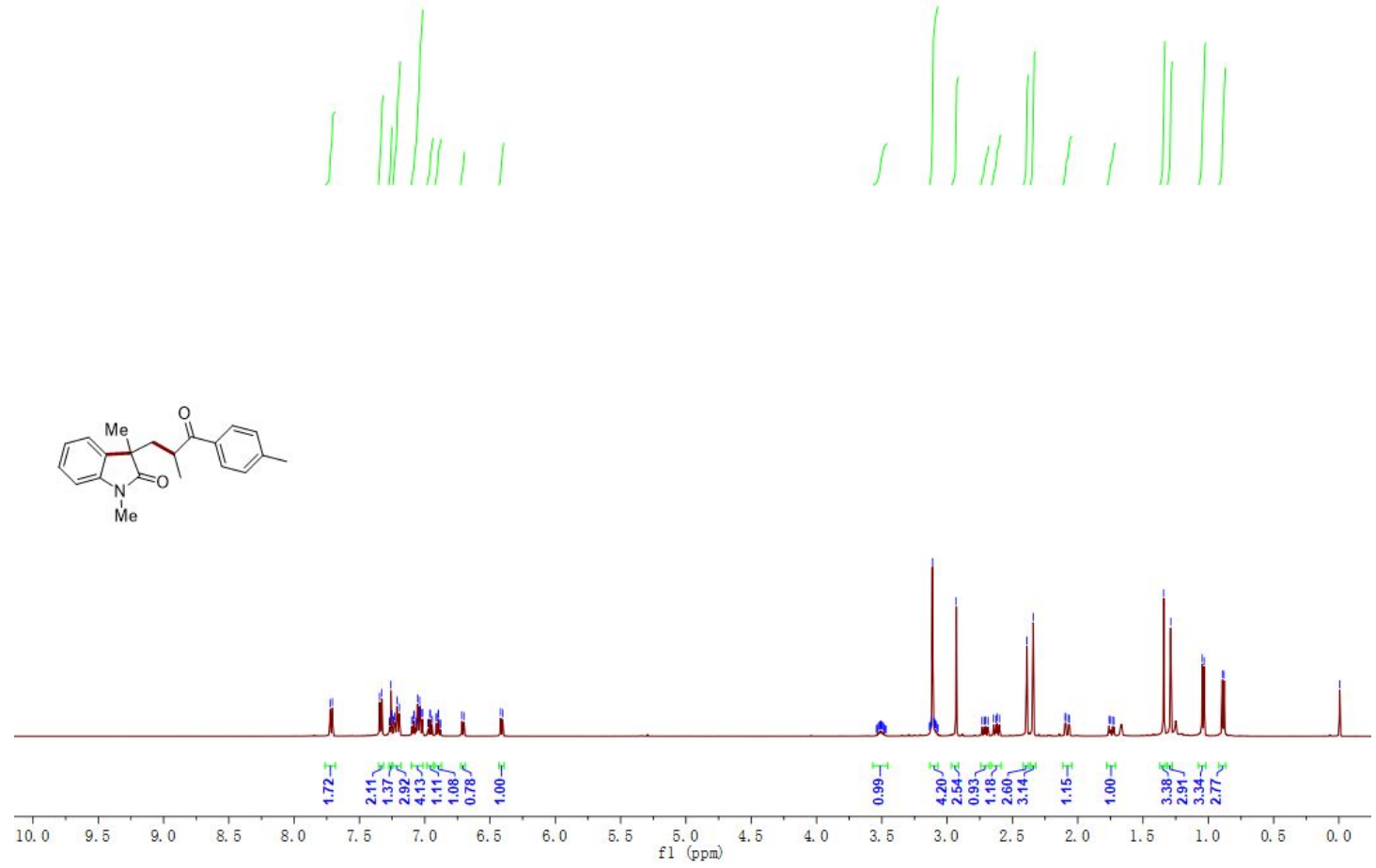

${ }^{13} \mathrm{C}\left\{{ }^{1} \mathrm{H}\right\}$ NMR of 3 ak $\left(125 \mathrm{MHz}, \mathrm{CDCl}_{3}\right)$

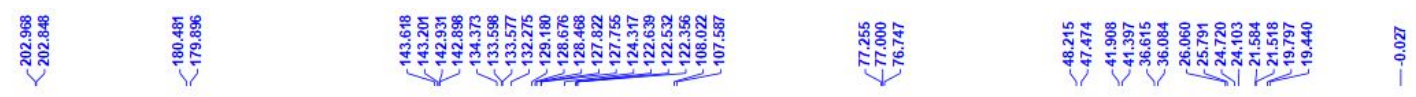
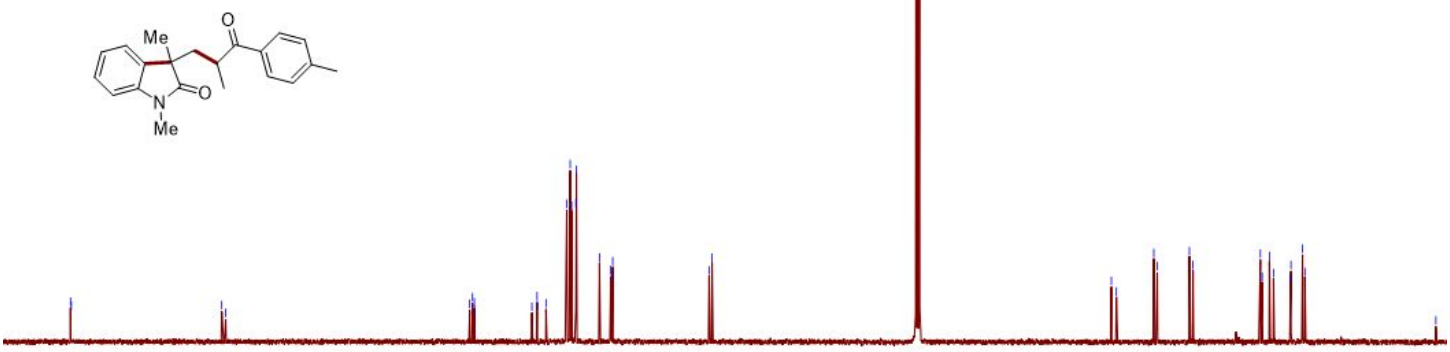

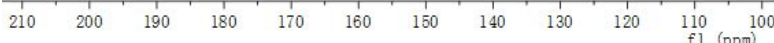

$\begin{array}{llllllllll}90 & 80 & 70 & 60 & 50 & 40 & 30 & 20 & 10 & 0\end{array}$ 
${ }^{1} \mathrm{H}$ NMR of 3al $\left(500 \mathrm{MHz}, \mathrm{CDCl}_{3}\right)$

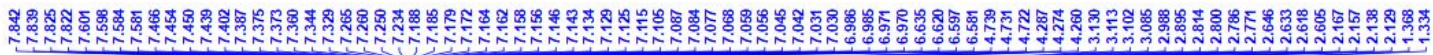
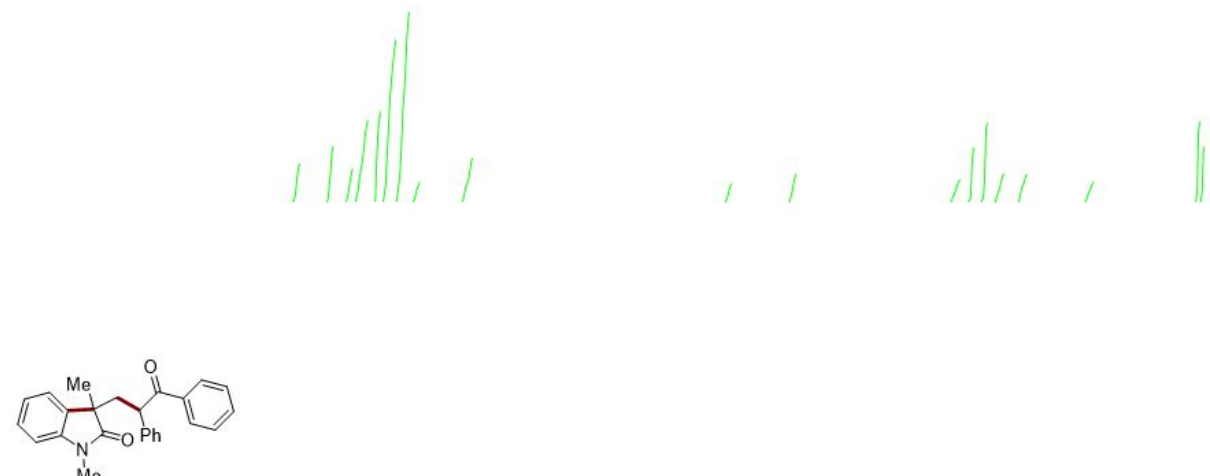

Me

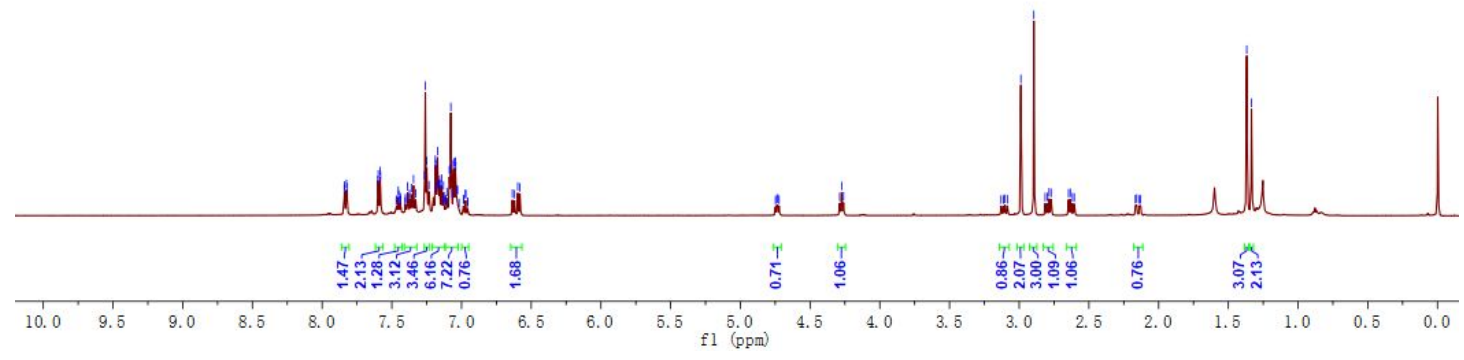

${ }^{13} \mathrm{C}\left\{{ }^{1} \mathrm{H}\right\}$ NMR of $\mathbf{3 a l}\left(125 \mathrm{MHz}, \mathrm{CDCl}_{3}\right)$
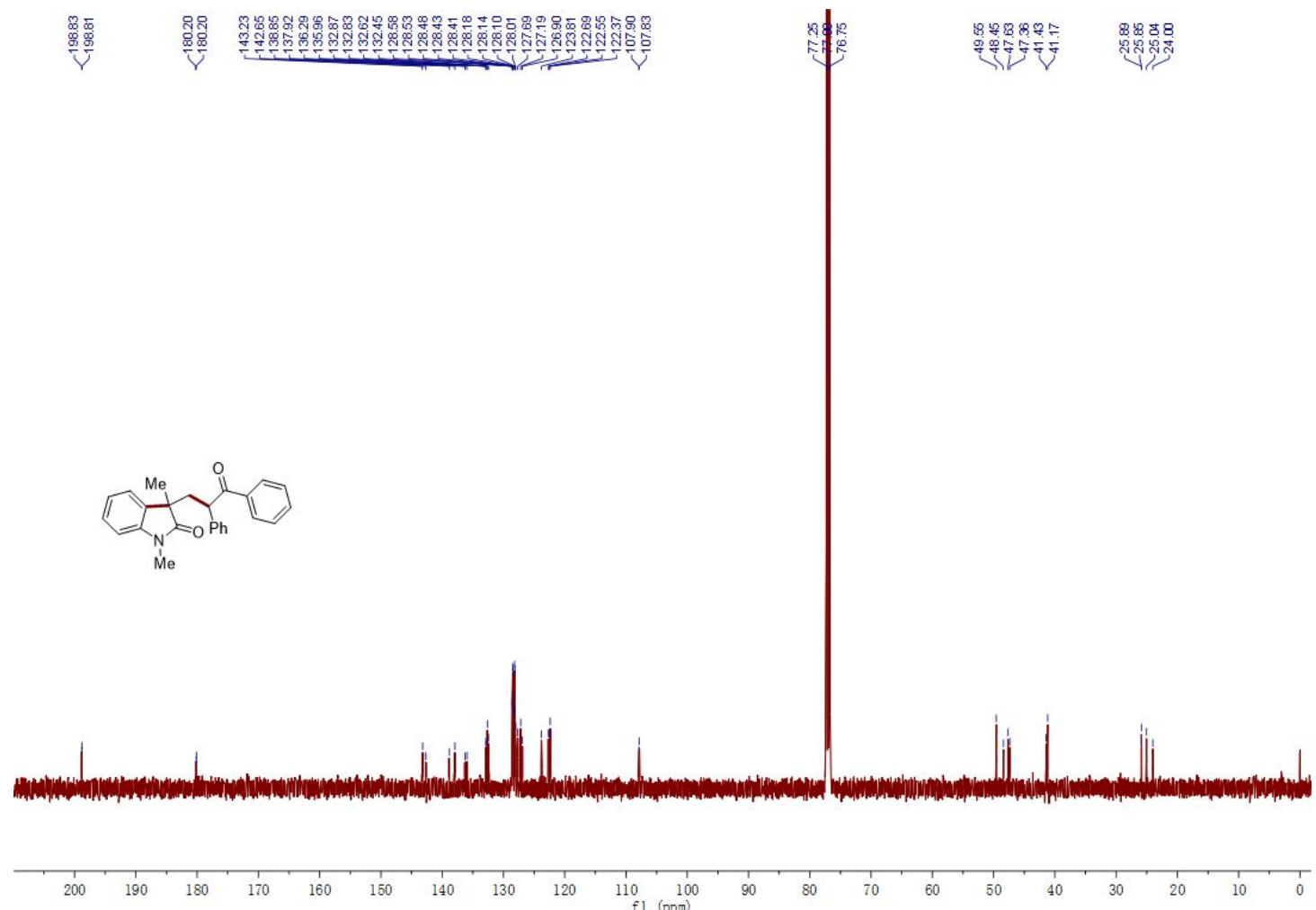
${ }^{1} \mathrm{H}$ NMR of 3am-1 (600 MHz, $\left.\mathrm{CDCl}_{3}\right)$

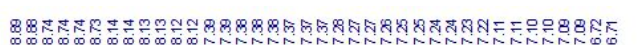

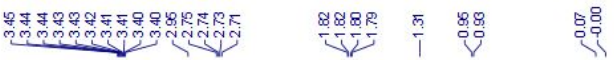

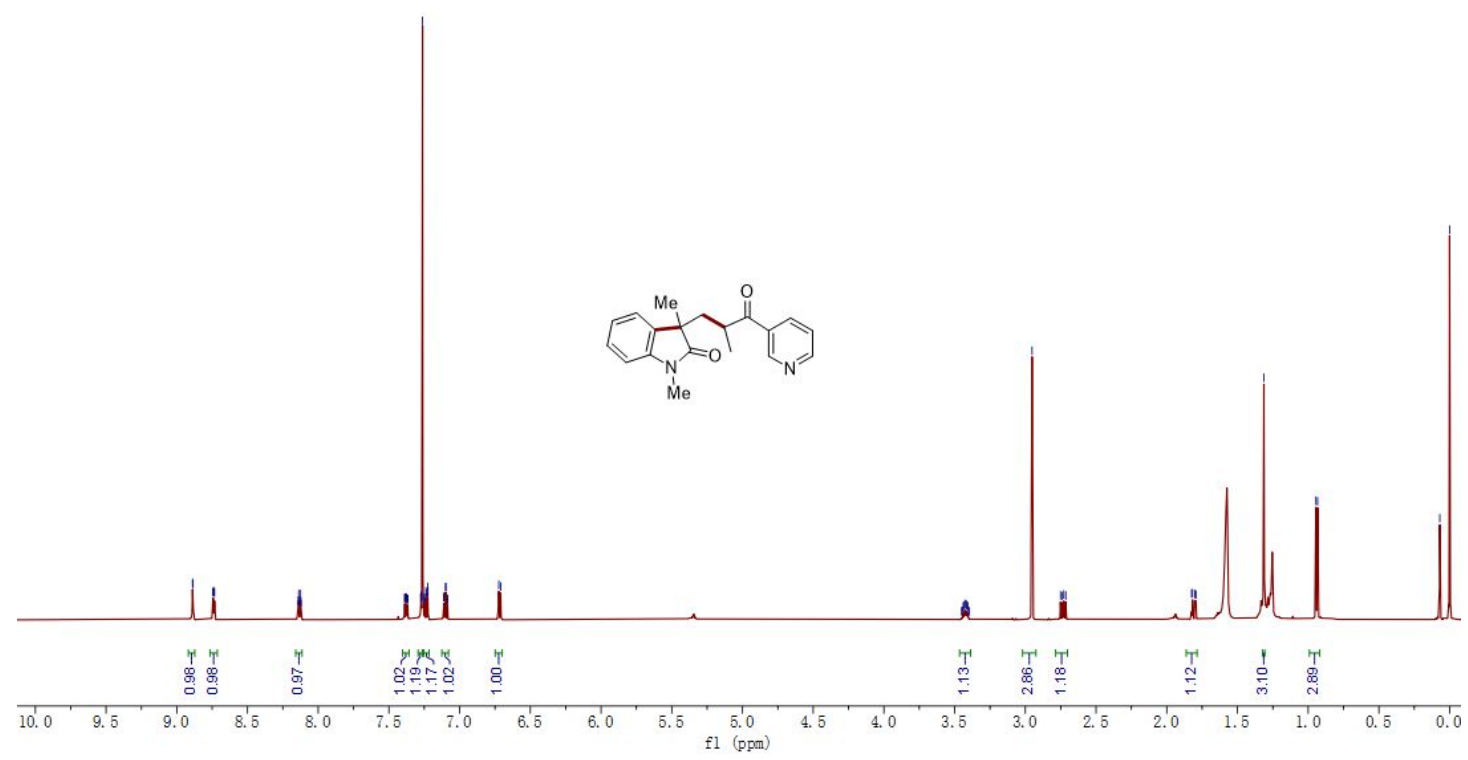

${ }^{13} \mathrm{C}\left\{{ }^{1} \mathrm{H}\right\}$ NMR of 3am-1 (150 MHz, $\left.\mathrm{CDCl}_{3}\right)$

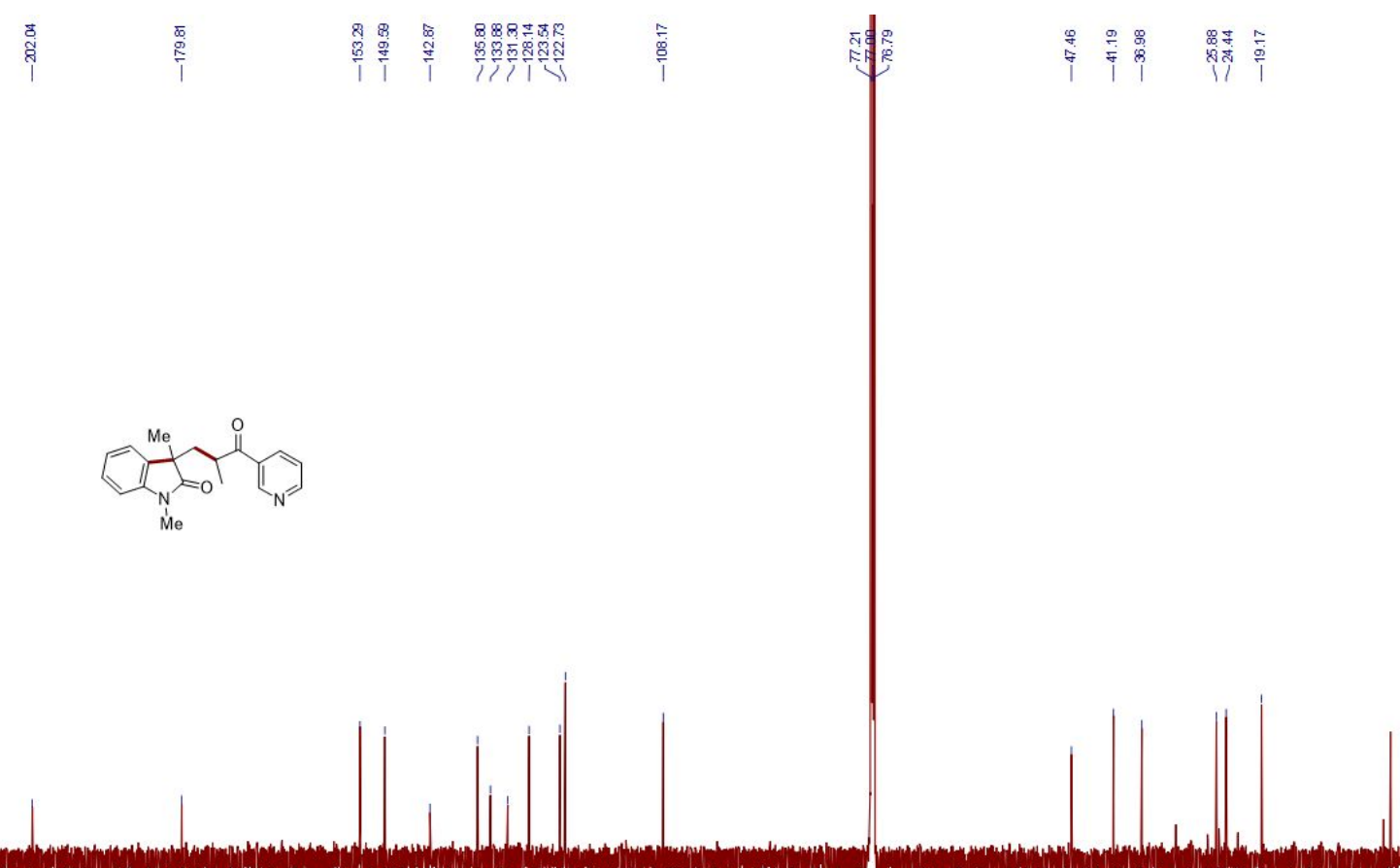

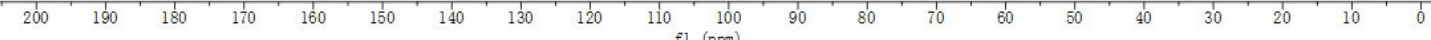


${ }^{1} \mathrm{H}$ NMR of 3am-2 (600 MHz, $\left.\mathrm{CDCl}_{3}\right)$

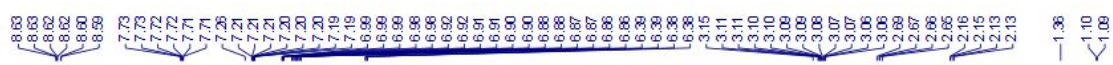

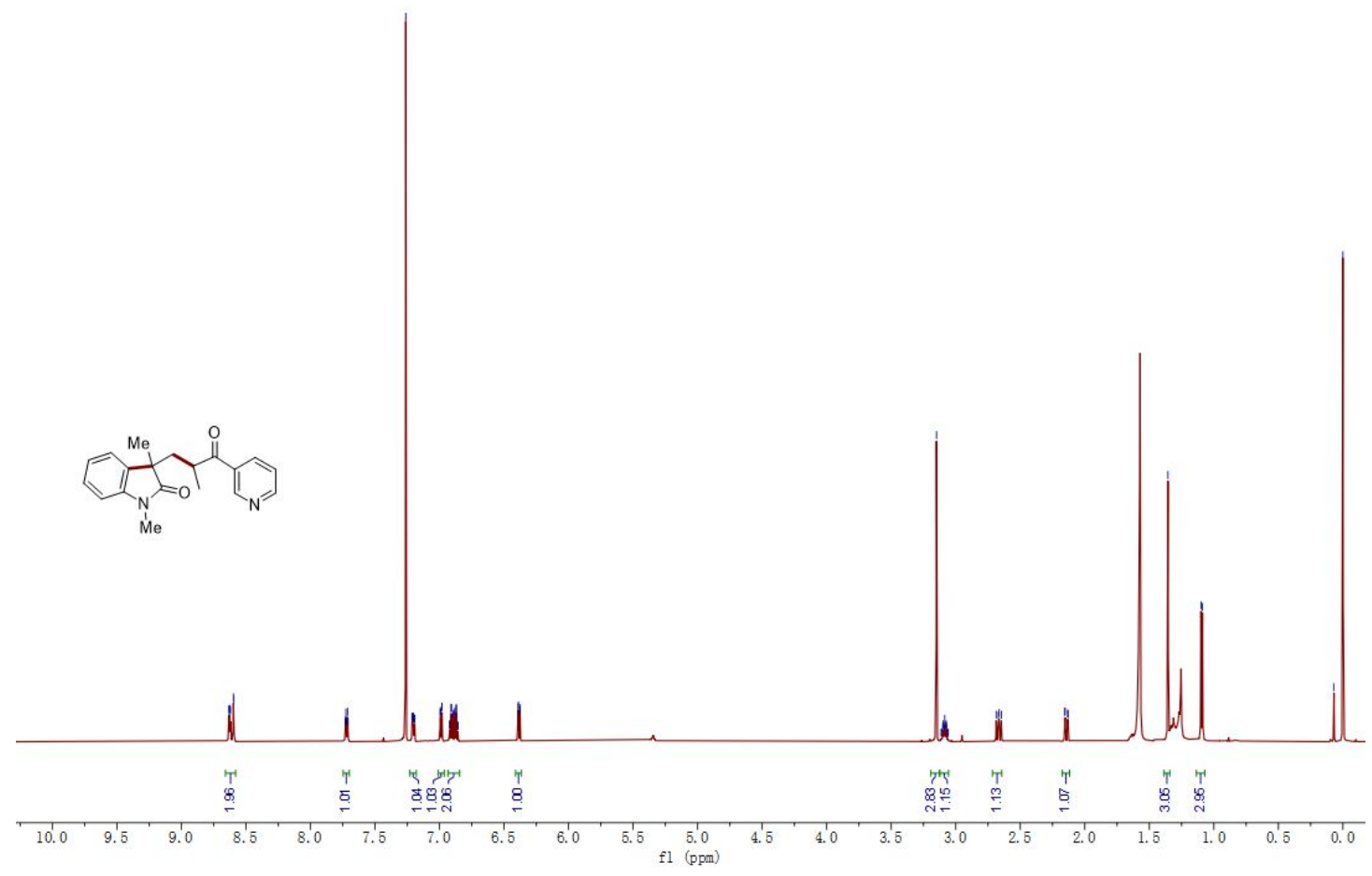

${ }^{13} \mathrm{C}\left\{{ }^{1} \mathrm{H}\right\}$ NMR of $\mathbf{3 a m}-\mathbf{2}\left(150 \mathrm{MHz}, \mathrm{CDCl}_{3}\right)$

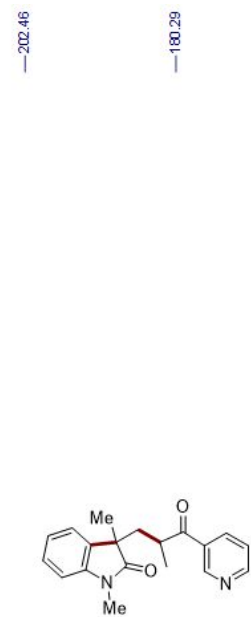

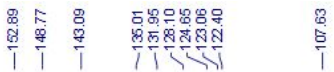

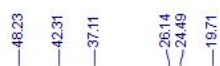

㡖

1 1 मा

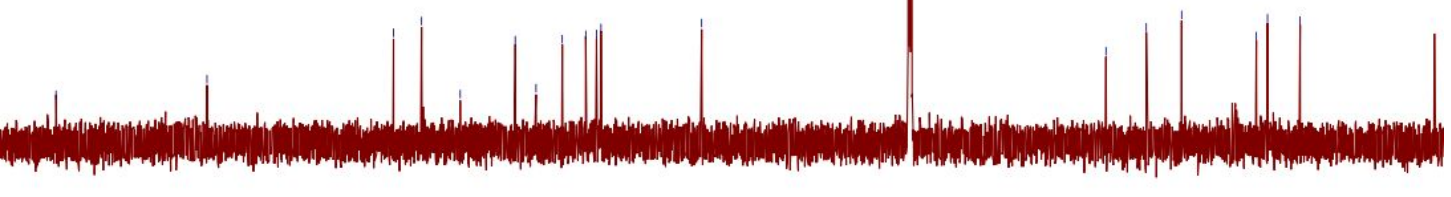

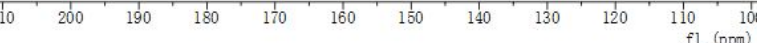

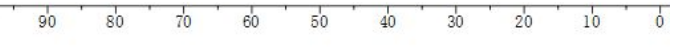


${ }^{1} \mathrm{H}$ NMR of 3 an $\left(500 \mathrm{MHz}, \mathrm{CDCl}_{3}\right)$

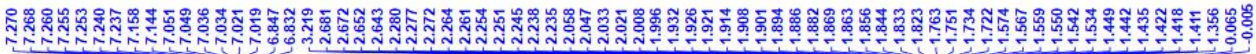
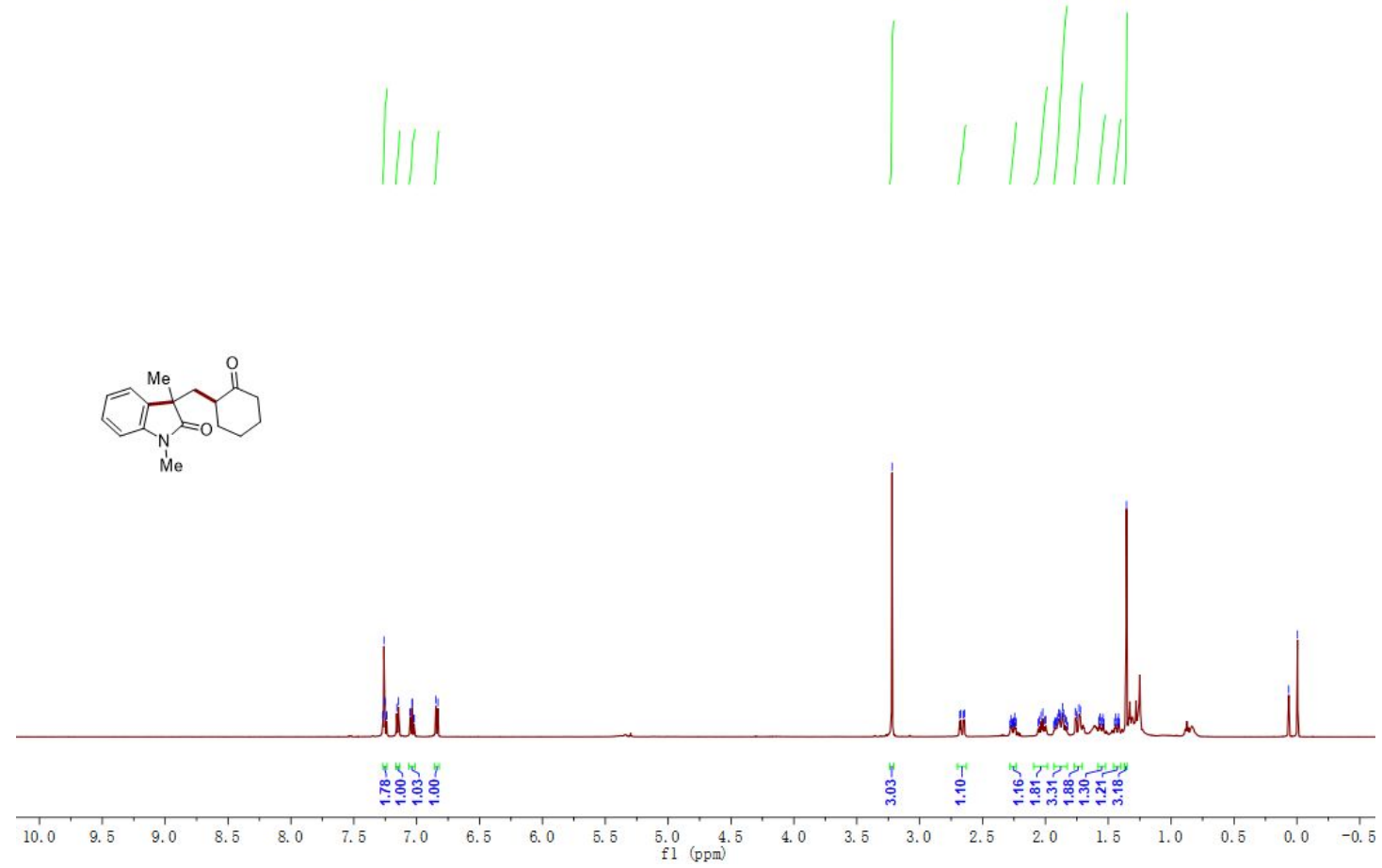

${ }^{13} \mathrm{C}\left\{{ }^{1} \mathrm{H}\right\}$ NMR of 3 an $\left(125 \mathrm{MHz}, \mathrm{CDCl}_{3}\right)$

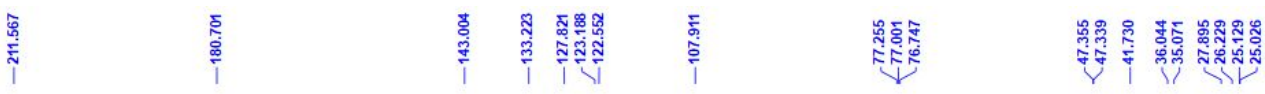

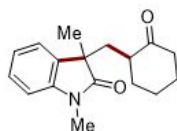

$\mathrm{Me}$

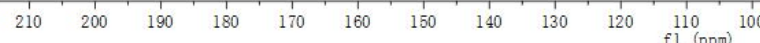

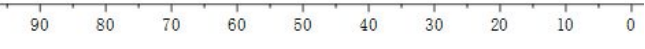


${ }^{1} \mathrm{H}$ NMR of $3 \mathbf{a o}\left(600 \mathrm{MHz}, \mathrm{CDCl}_{3}\right)$

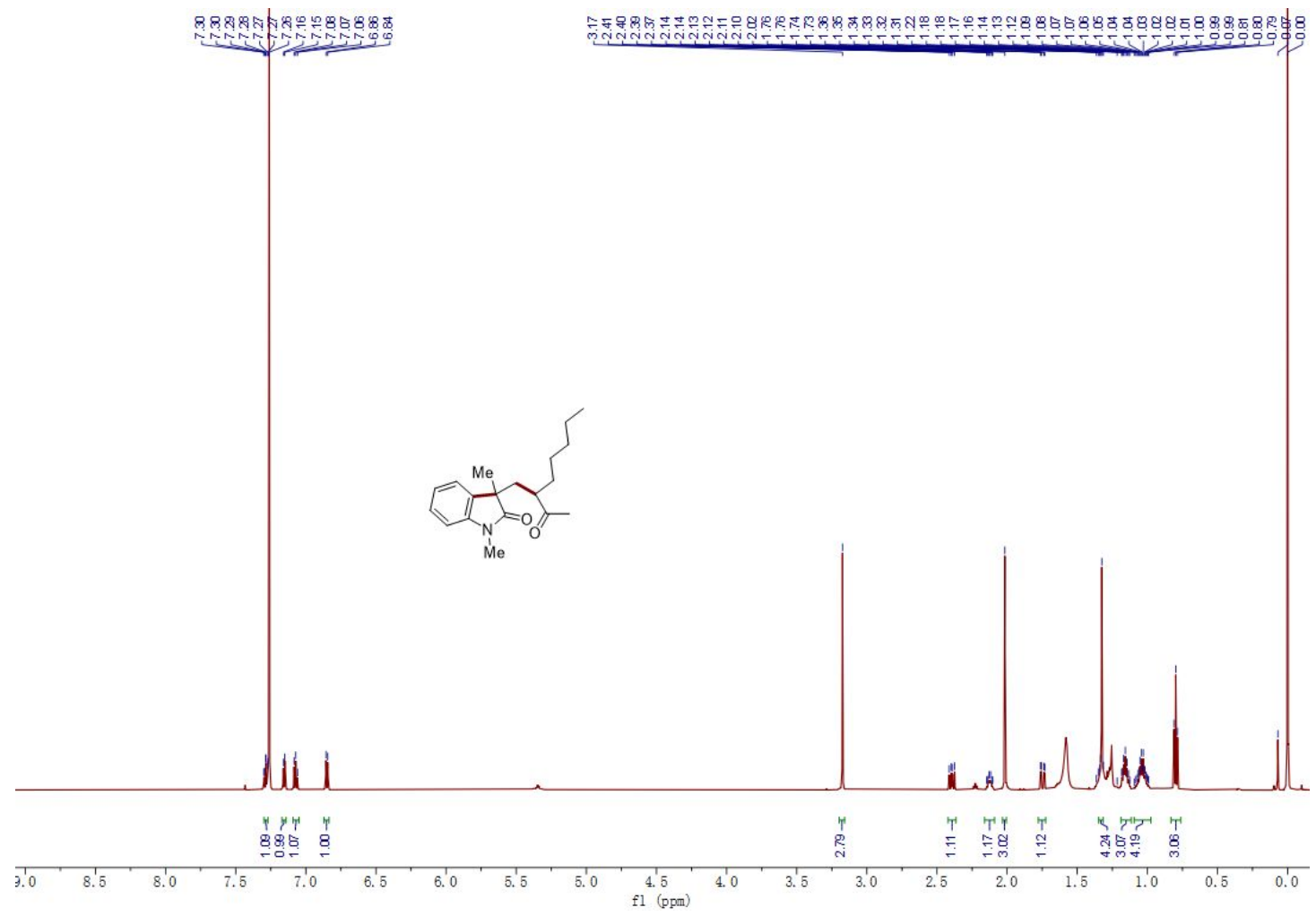

${ }^{13} \mathrm{C}\left\{{ }^{1} \mathrm{H}\right\}$ NMR of $\mathbf{3 a o}\left(150 \mathrm{MHz}, \mathrm{CDCl}_{3}\right)$

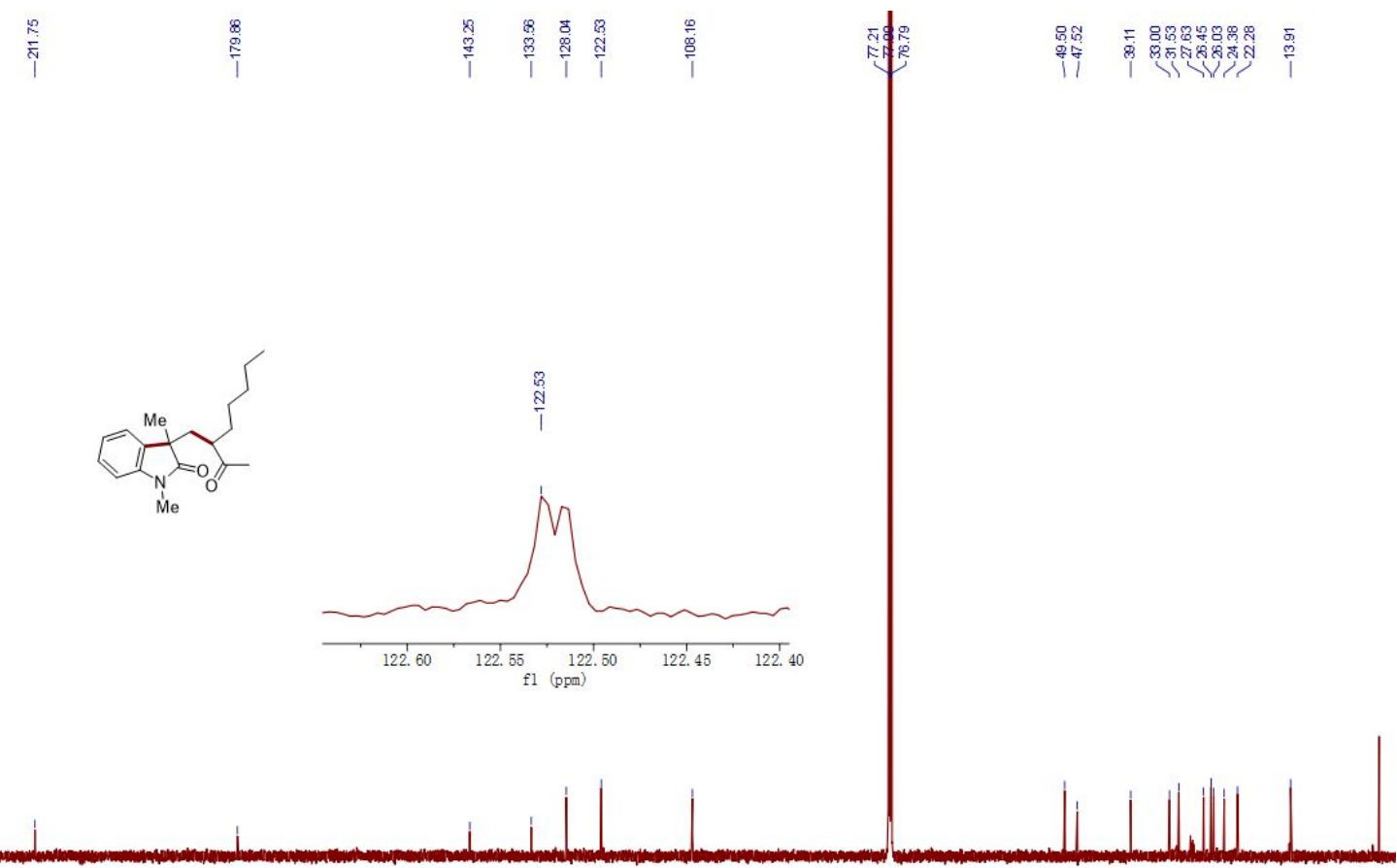

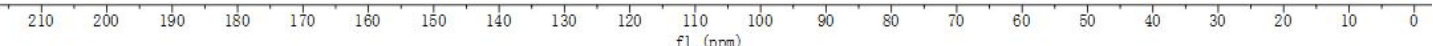


${ }^{1} \mathrm{H}$ NMR of 3 ap $\left(500 \mathrm{MHz}, \mathrm{CDCl}_{3}\right)$

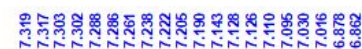

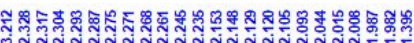

응융
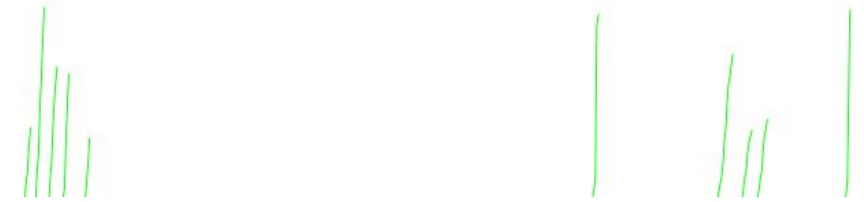

$\underbrace{M e}_{\substack{N \\ \text { Me }}}$

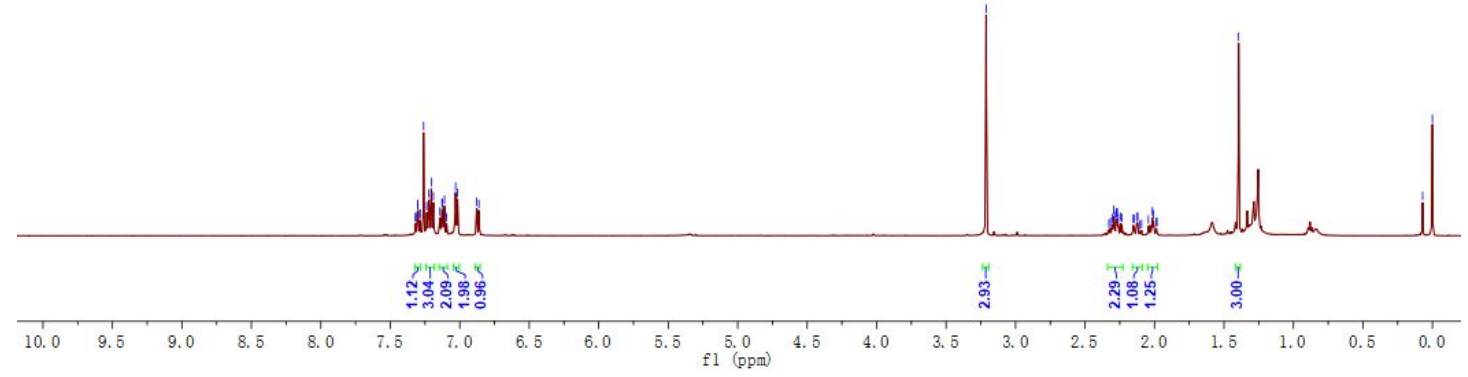

${ }^{13} \mathrm{C}\left\{{ }^{1} \mathrm{H}\right\}$ NMR of 3 ap $\left(125 \mathrm{MHz}, \mathrm{CDCl}_{3}\right)$

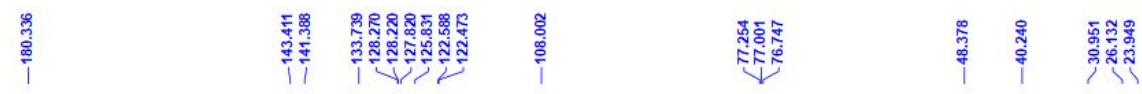
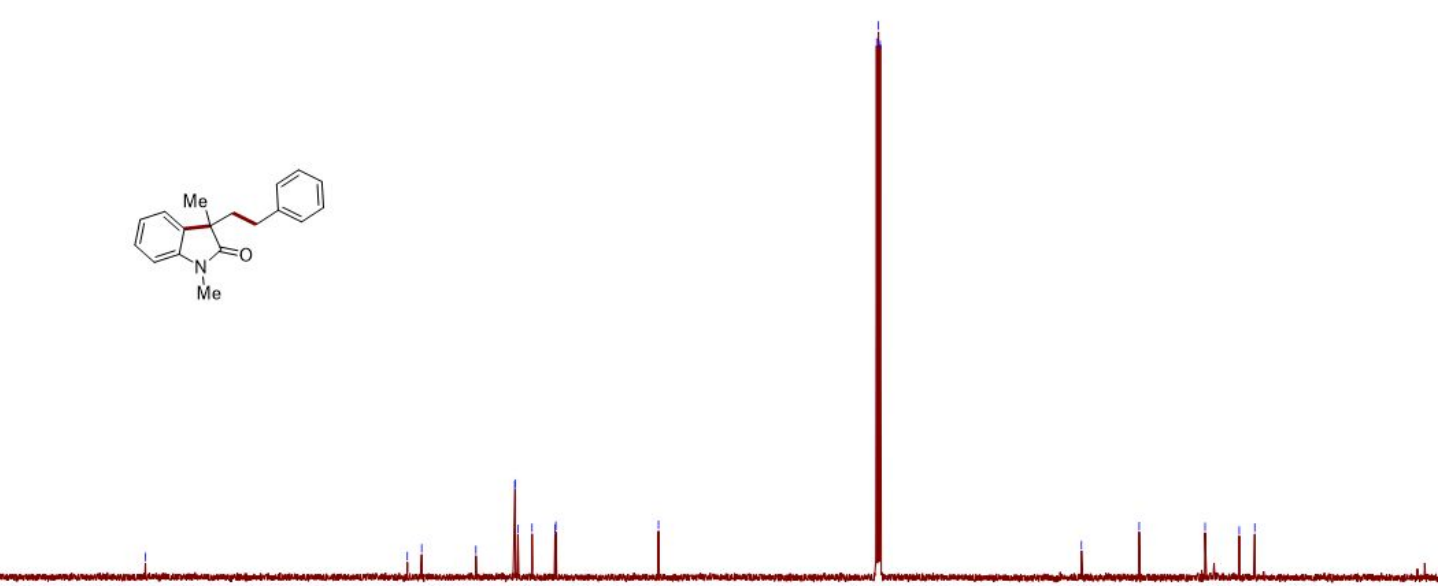

${ }_{200}^{T}$

200

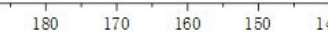

${ }_{130}^{1} 120$

$10 \underset{\mathrm{fl}}{100}(\mathrm{ppm})$

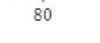


${ }^{1} \mathrm{H}$ NMR of $\mathbf{3 a q}\left(500 \mathrm{MHz}, \mathrm{CDCl}_{3}\right)$

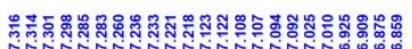

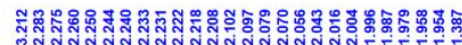

I-

ō
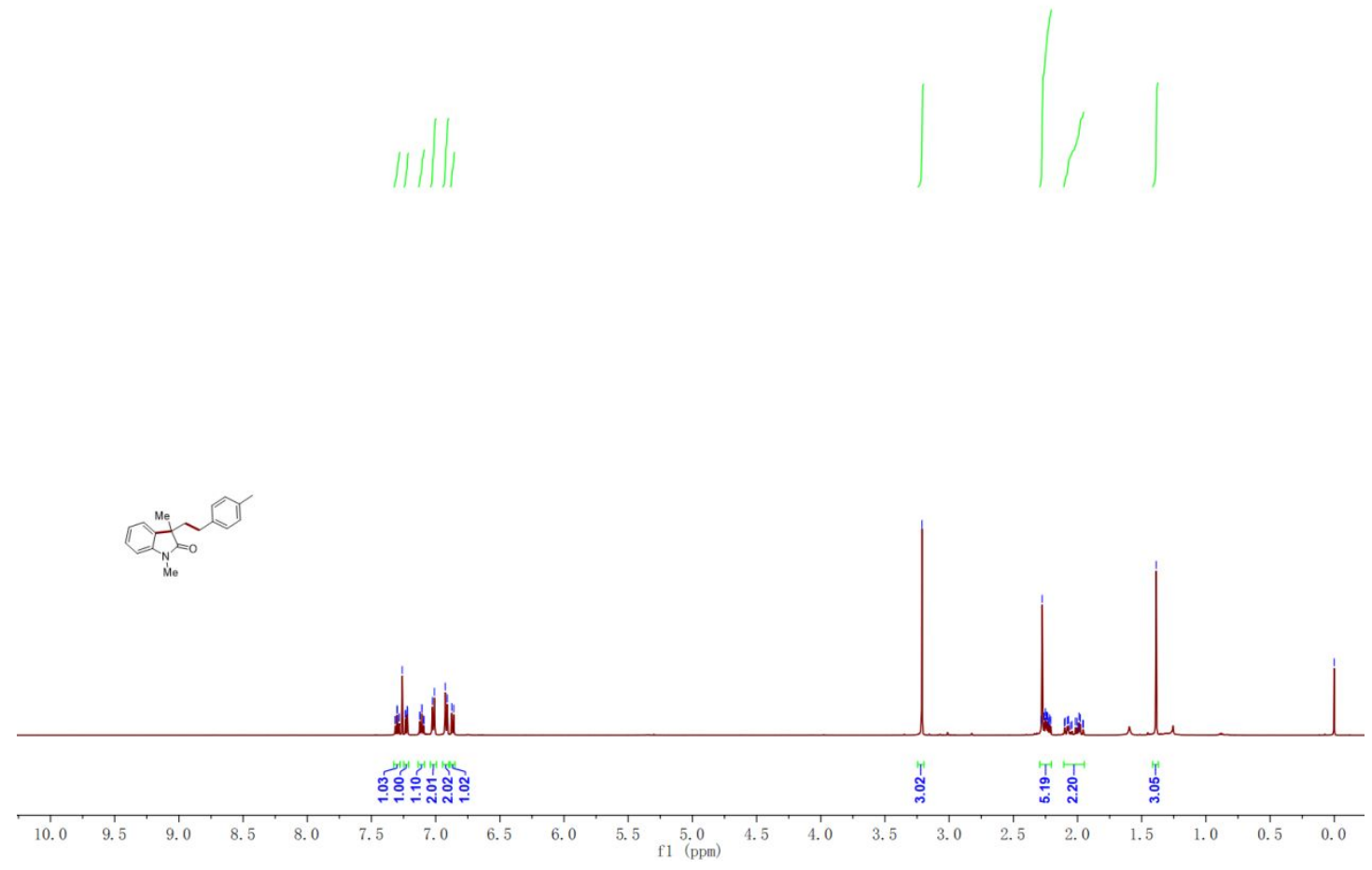

${ }^{13} \mathrm{C}\left\{{ }^{1} \mathrm{H}\right\}$ NMR of $\mathbf{3 a q}\left(125 \mathrm{MHz}, \mathrm{CDCl}_{3}\right)$

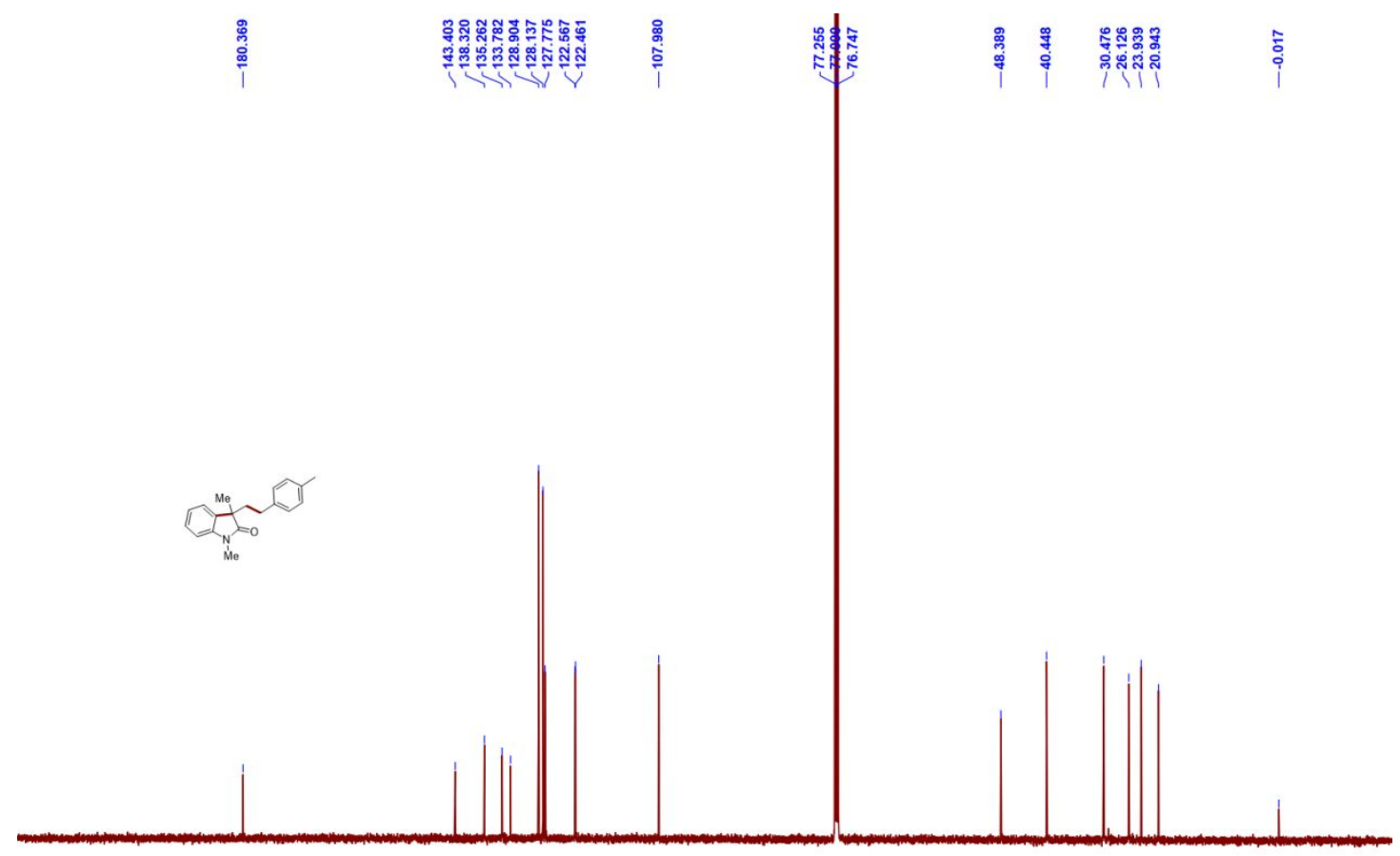

$\begin{array}{llllllllllllllllllllllllllllllll}1 & 1 \\ 210 & 200 & 190 & 180 & 170 & 160 & 150 & 140 & 130 & 120 & 110 & 100 & 90 & 80 & 70 & 60 & 50 & 40 & 30 & 20 & 10 & 0 & -10\end{array}$ 
${ }^{1} \mathrm{H} \mathrm{NMR}$ of $3 \mathbf{a r}\left(500 \mathrm{MHz}, \mathrm{CDCl}_{3}\right)$

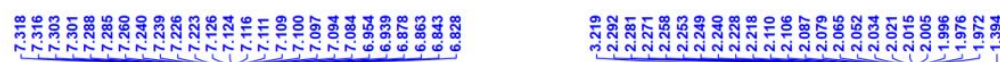

亏ั
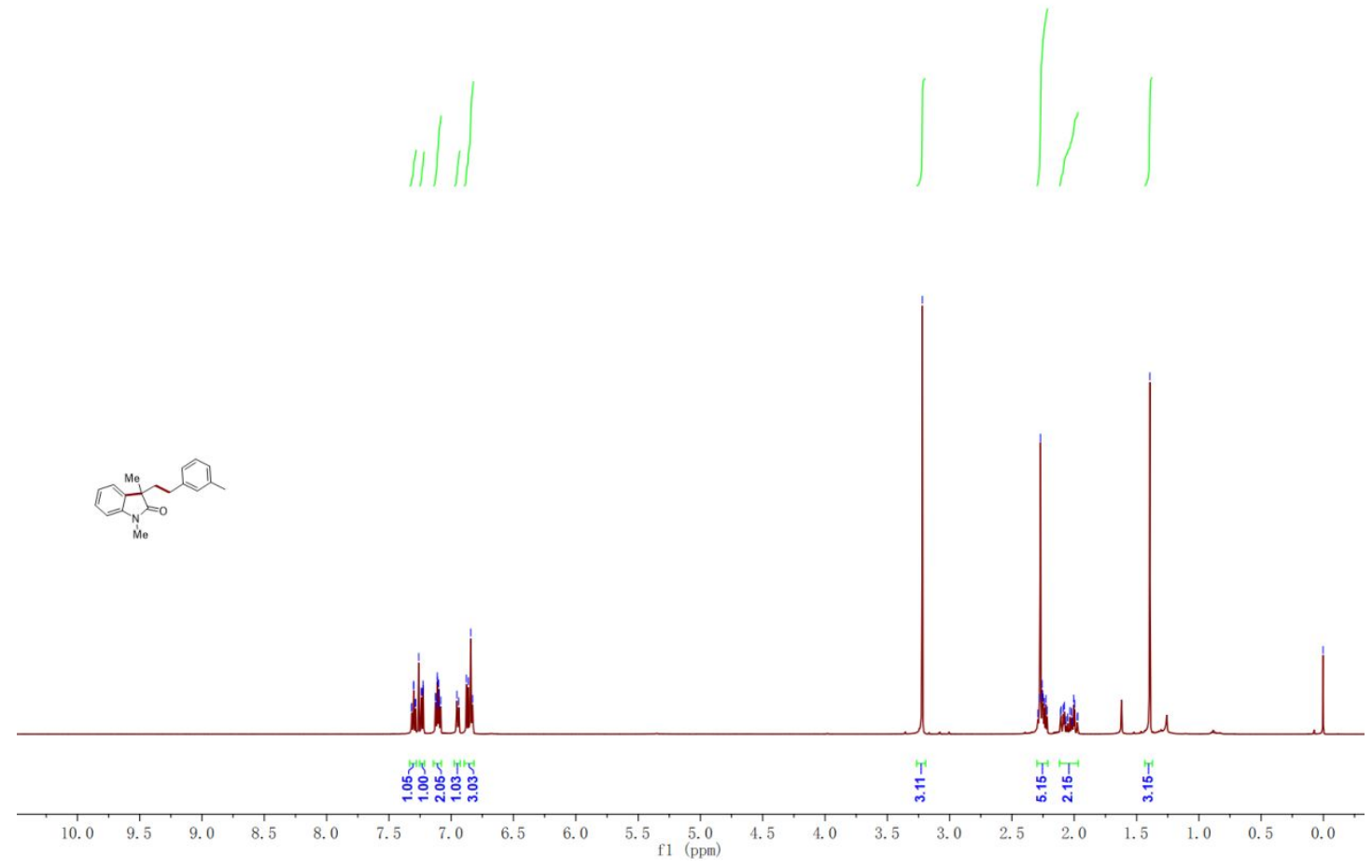

${ }^{13} \mathrm{C}\left\{{ }^{1} \mathrm{H}\right\}$ NMR of $3 \operatorname{ar}\left(125 \mathrm{MHz}, \mathrm{CDCl}_{3}\right)$

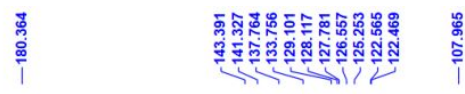
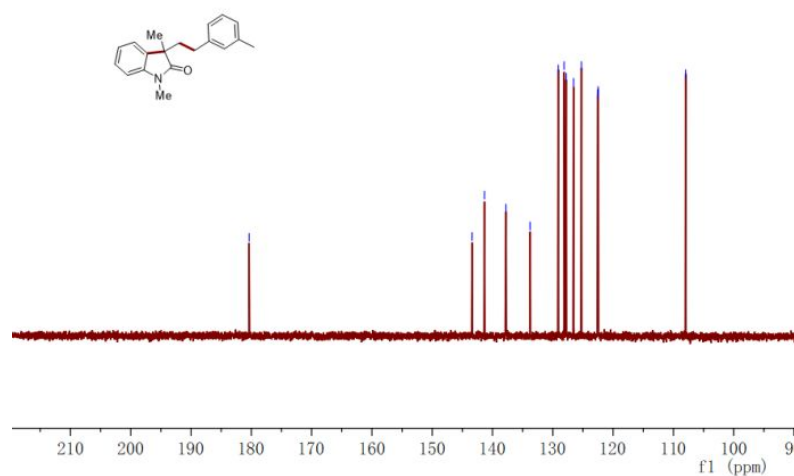
${ }^{1} \mathrm{H}$ NMR of 3 as $\left(500 \mathrm{MHz}, \mathrm{CDCl}_{3}\right)$

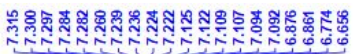

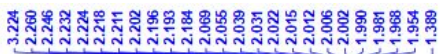

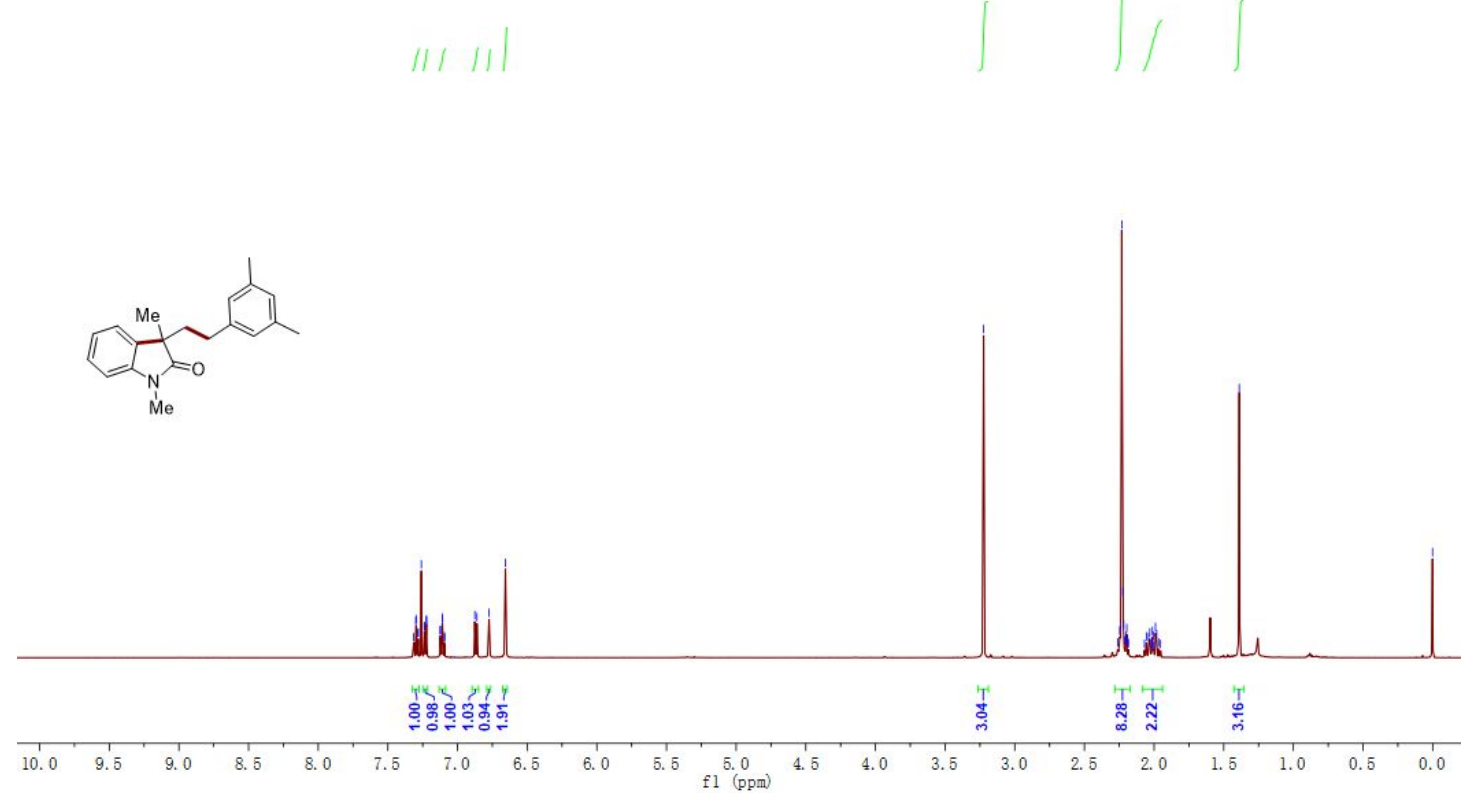

${ }^{13} \mathrm{C}\left\{{ }^{1} \mathrm{H}\right\}$ NMR of 3 as $\left(125 \mathrm{MHz}, \mathrm{CDCl}_{3}\right)$

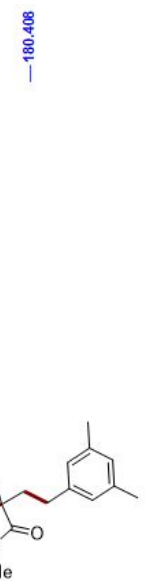

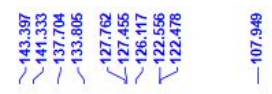

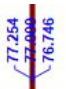

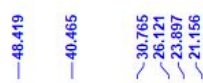
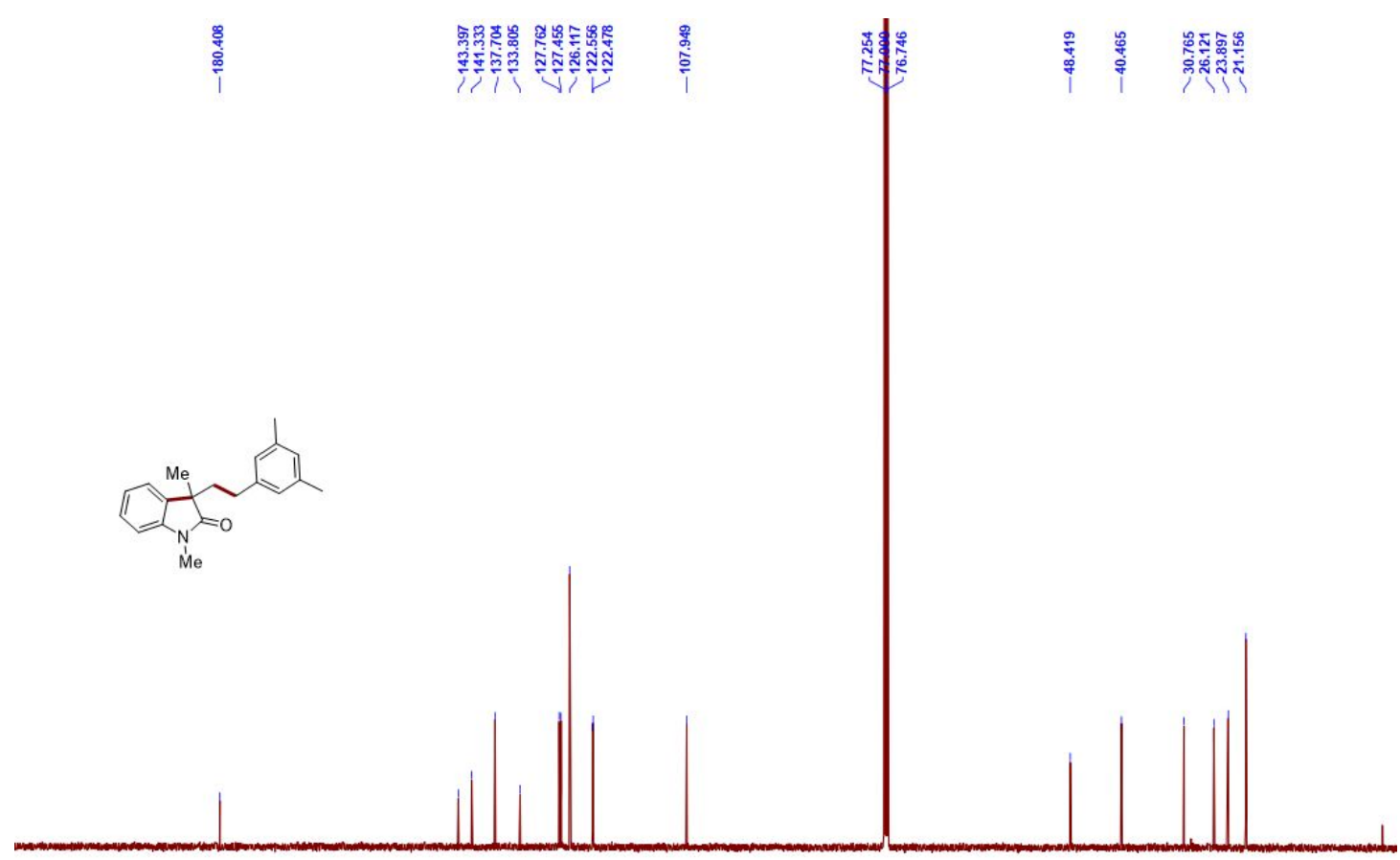

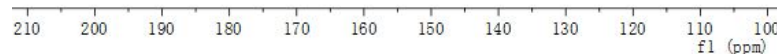




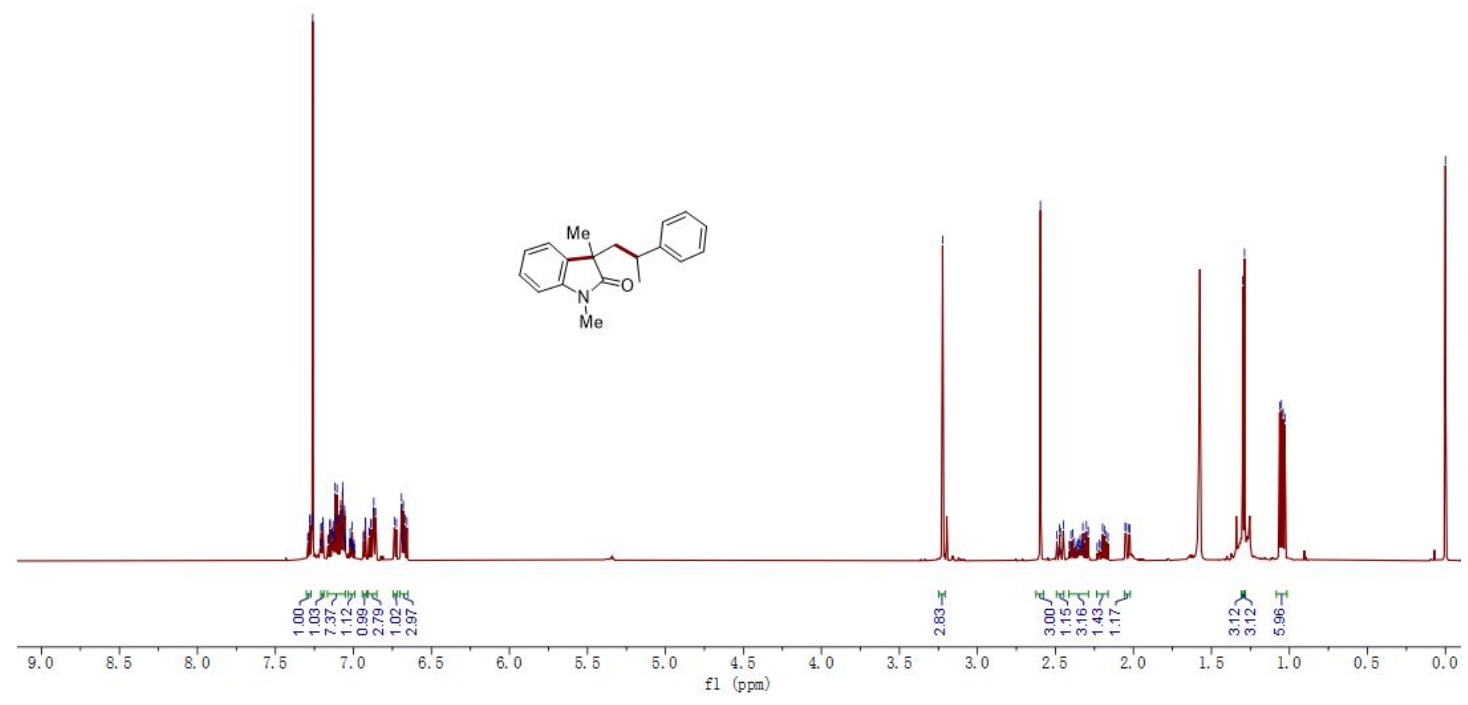

${ }^{13} \mathrm{C}\left\{{ }^{1} \mathrm{H}\right\}$ NMR of 3 at $\left(150 \mathrm{MHz}, \mathrm{CDCl}_{3}\right)$

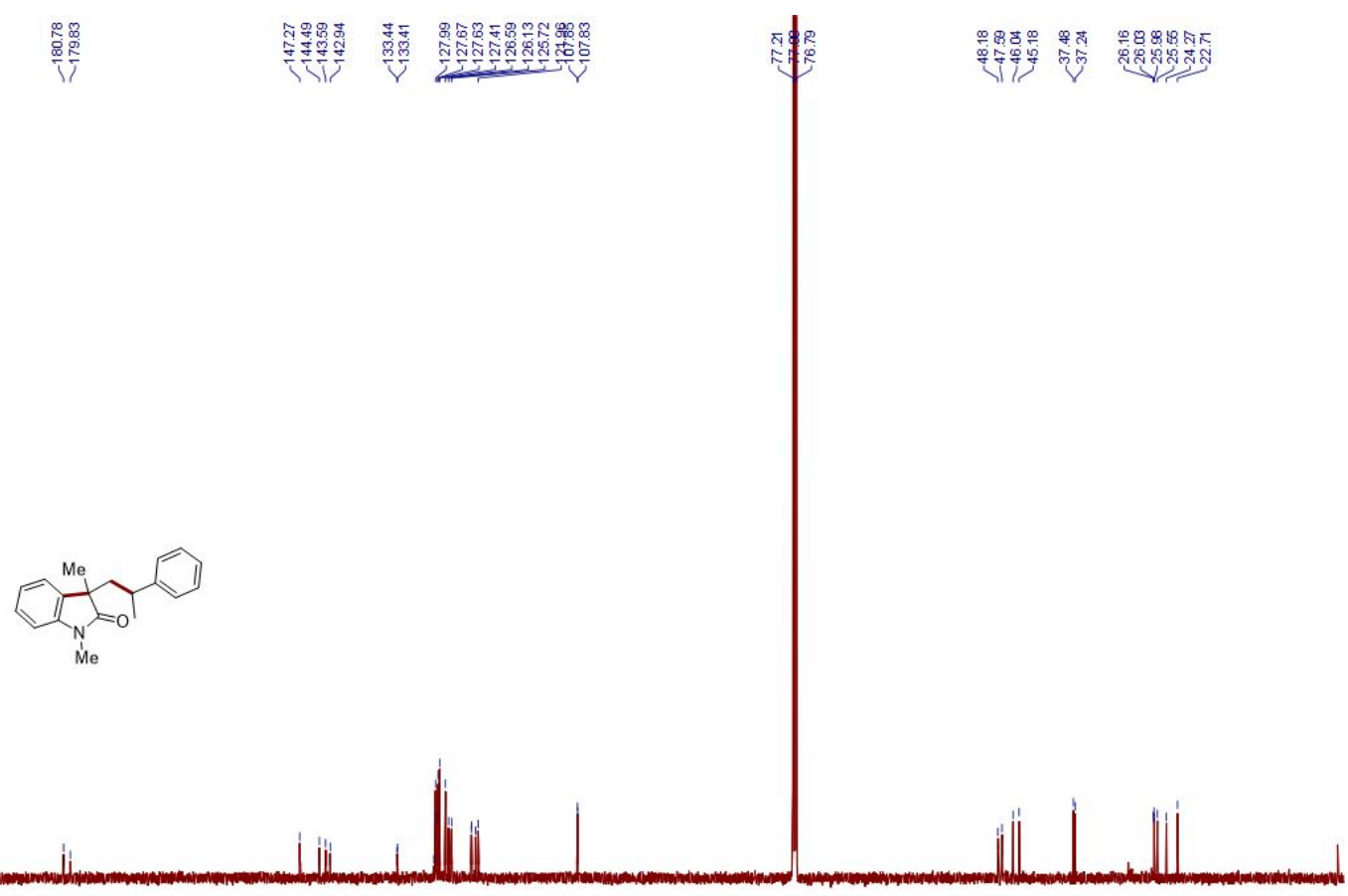

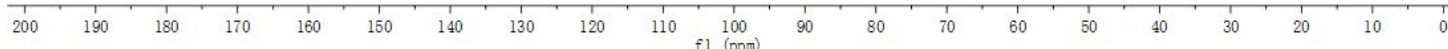


${ }^{1} \mathrm{H}$ NMR of 3au $\left(600 \mathrm{MHz}, \mathrm{CDCl}_{3}\right)$

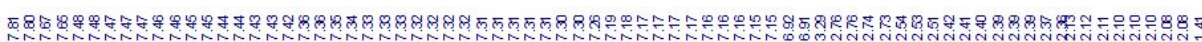
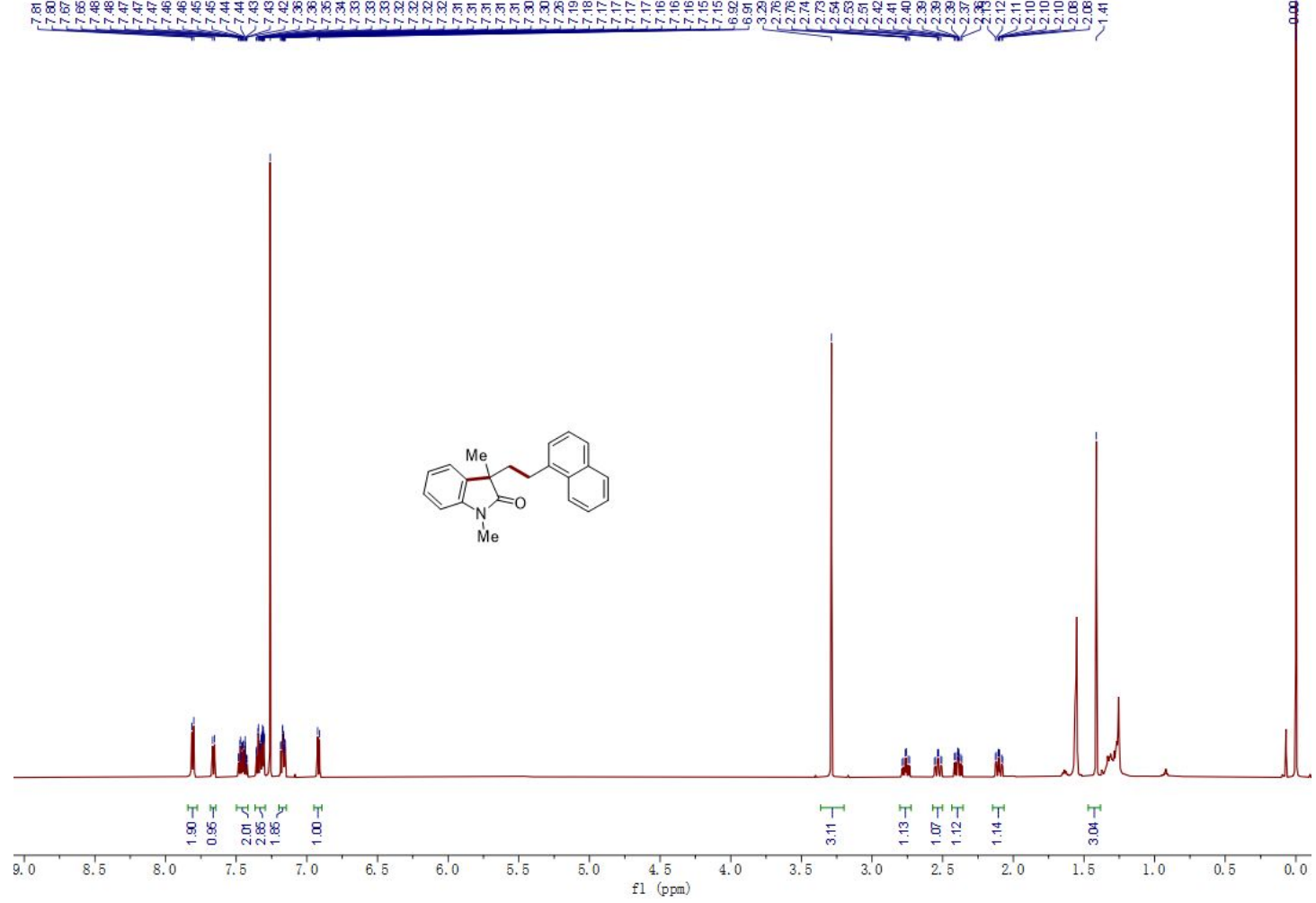

${ }^{13} \mathrm{C}\left\{{ }^{1} \mathrm{H}\right\}$ NMR of $\mathbf{3 a u}\left(150 \mathrm{MHz}, \mathrm{CDCl}_{3}\right)$

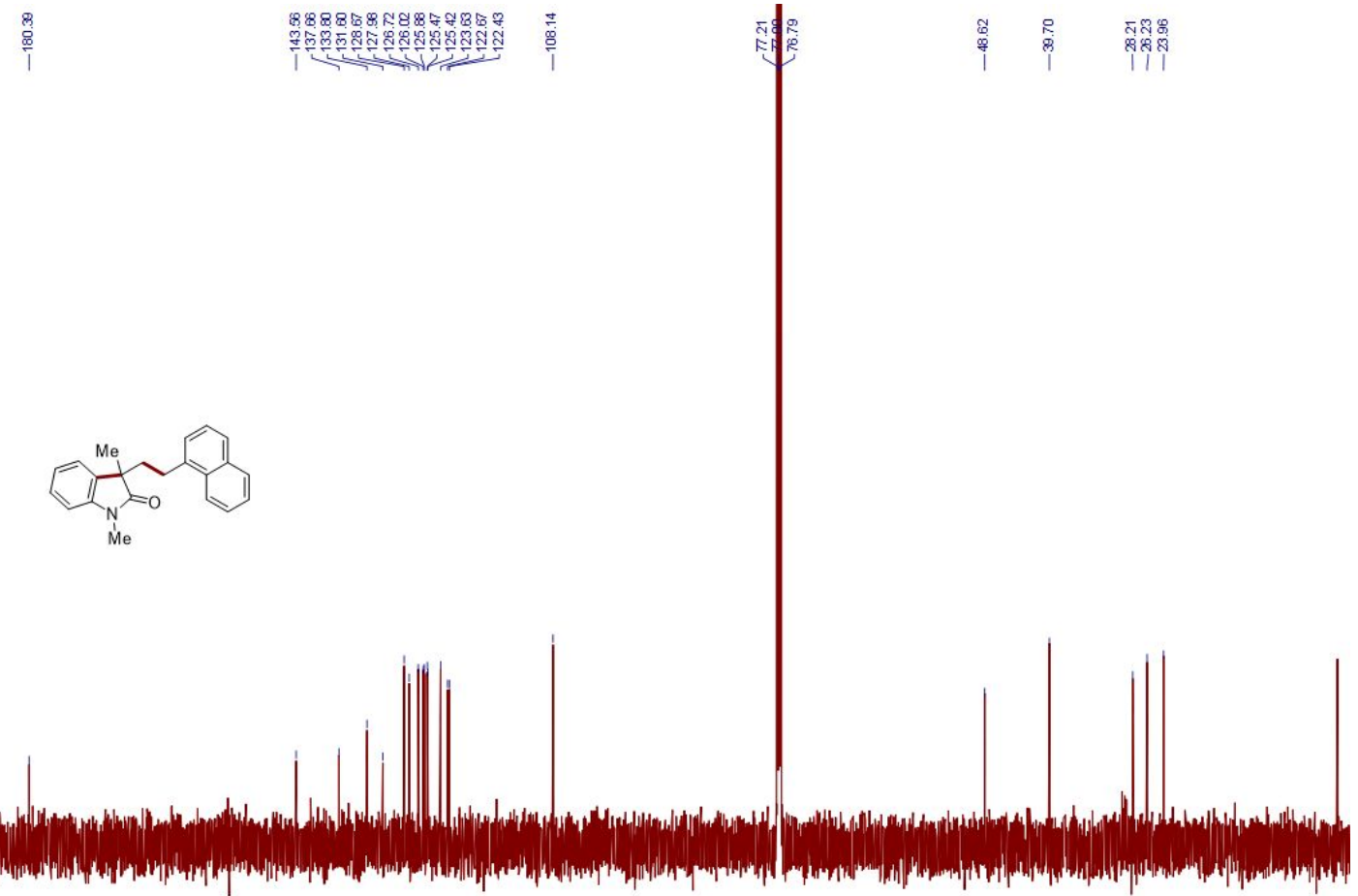

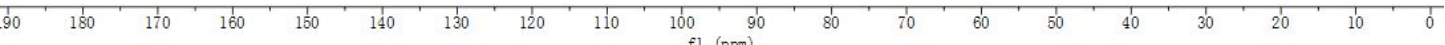


${ }^{1} \mathrm{H} \mathrm{NMR}$ of $3 \mathbf{a v}\left(500 \mathrm{MHz}, \mathrm{CDCl}_{3}\right)$

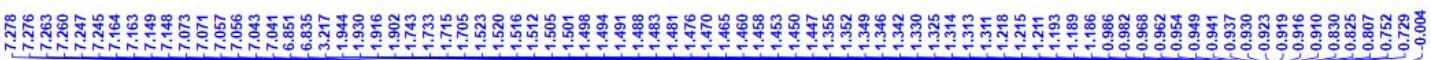

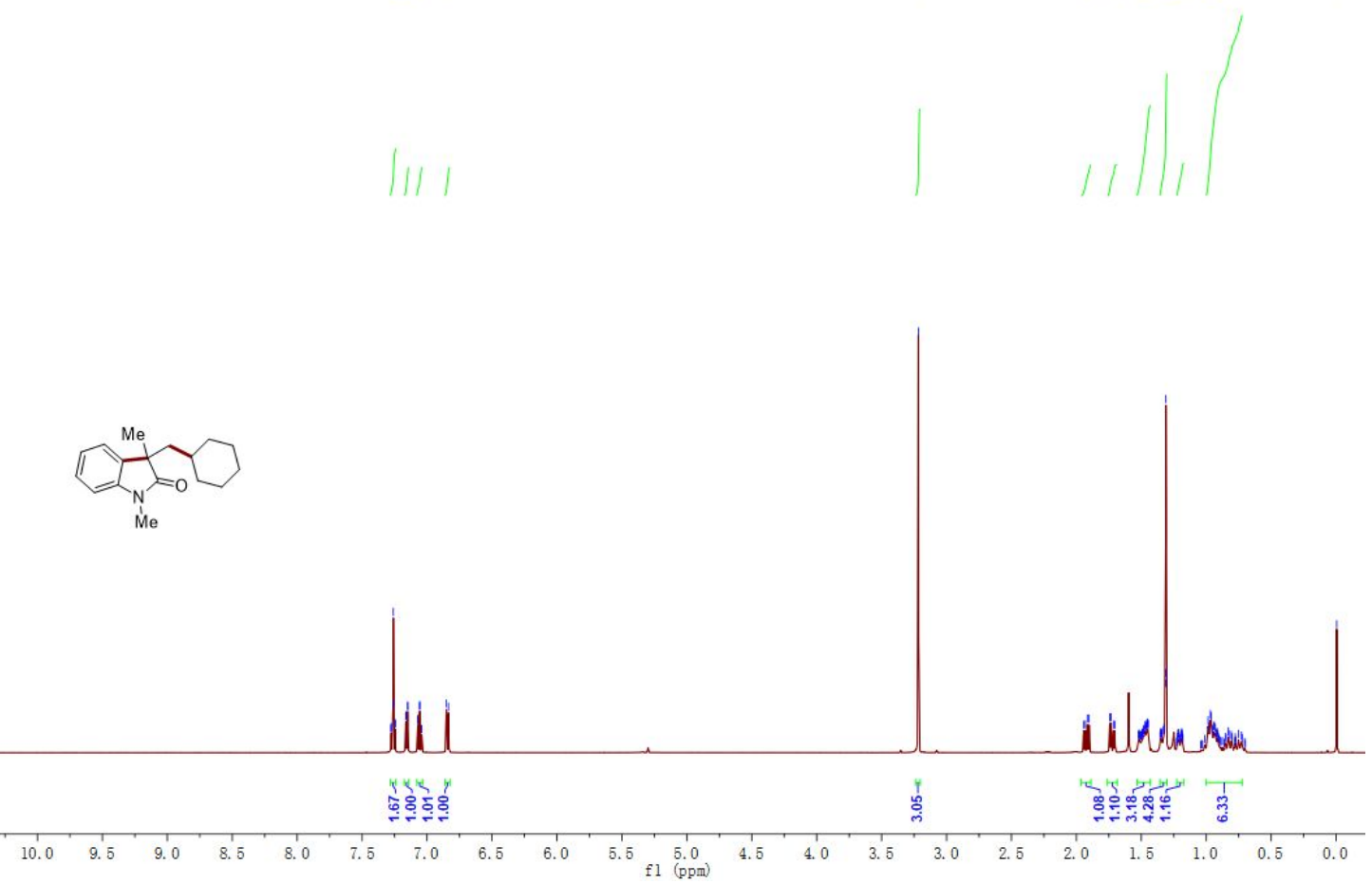

${ }^{13} \mathrm{C}\left\{{ }^{1} \mathrm{H}\right\}$ NMR of $\mathbf{3 a v}\left(125 \mathrm{MHz}, \mathrm{CDCl}_{3}\right)$

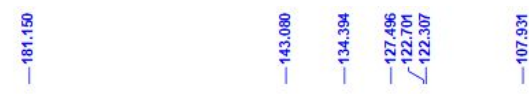

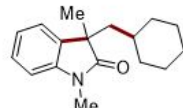

Me

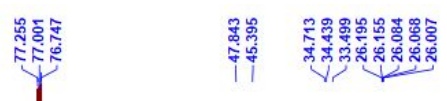

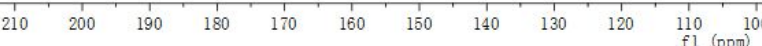


${ }^{1} \mathrm{H}$ NMR of 3aw $\left(600 \mathrm{MHz}, \mathrm{CDCl}_{3}\right)$

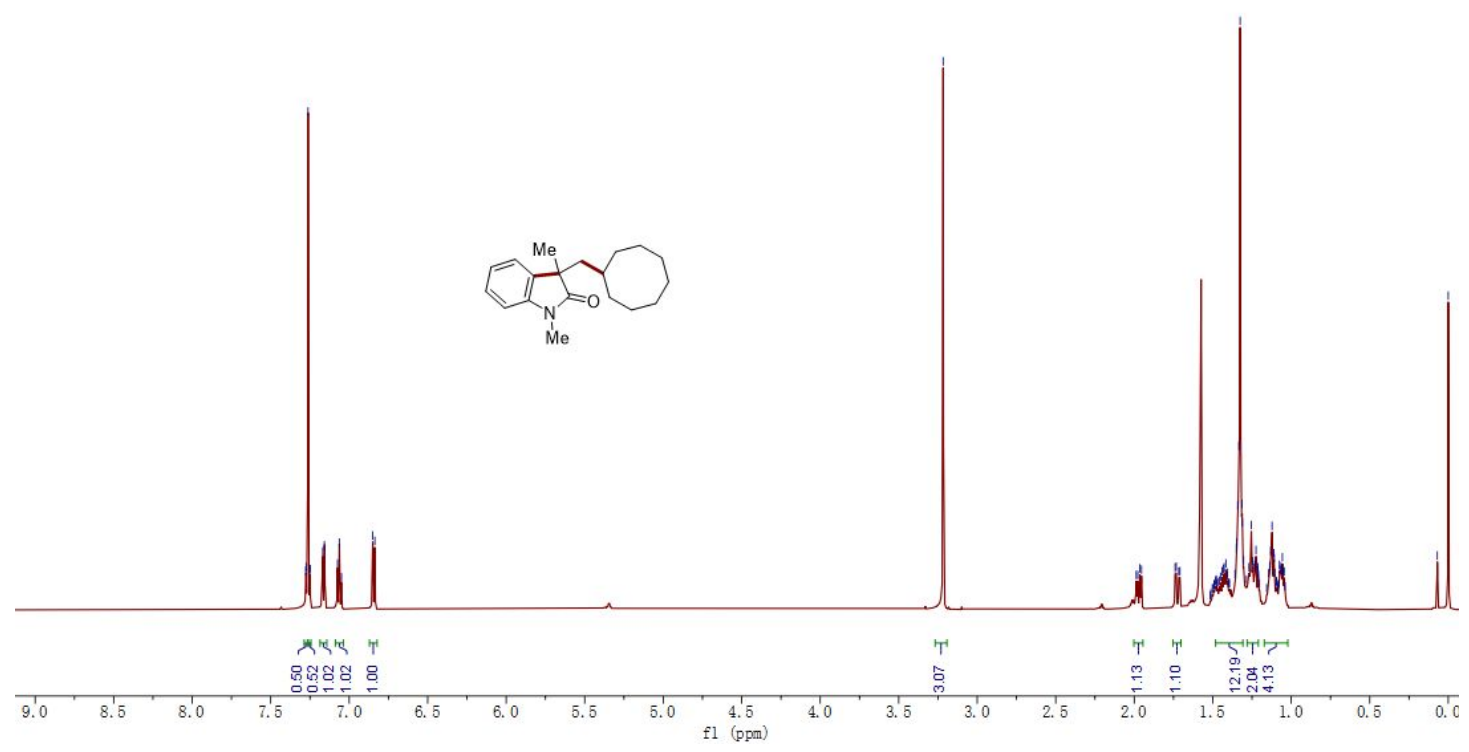

${ }^{13} \mathrm{C}\left\{{ }^{1} \mathrm{H}\right\}$ NMR of 3 aw $\left(150 \mathrm{MHz}, \mathrm{CDCl}_{3}\right)$
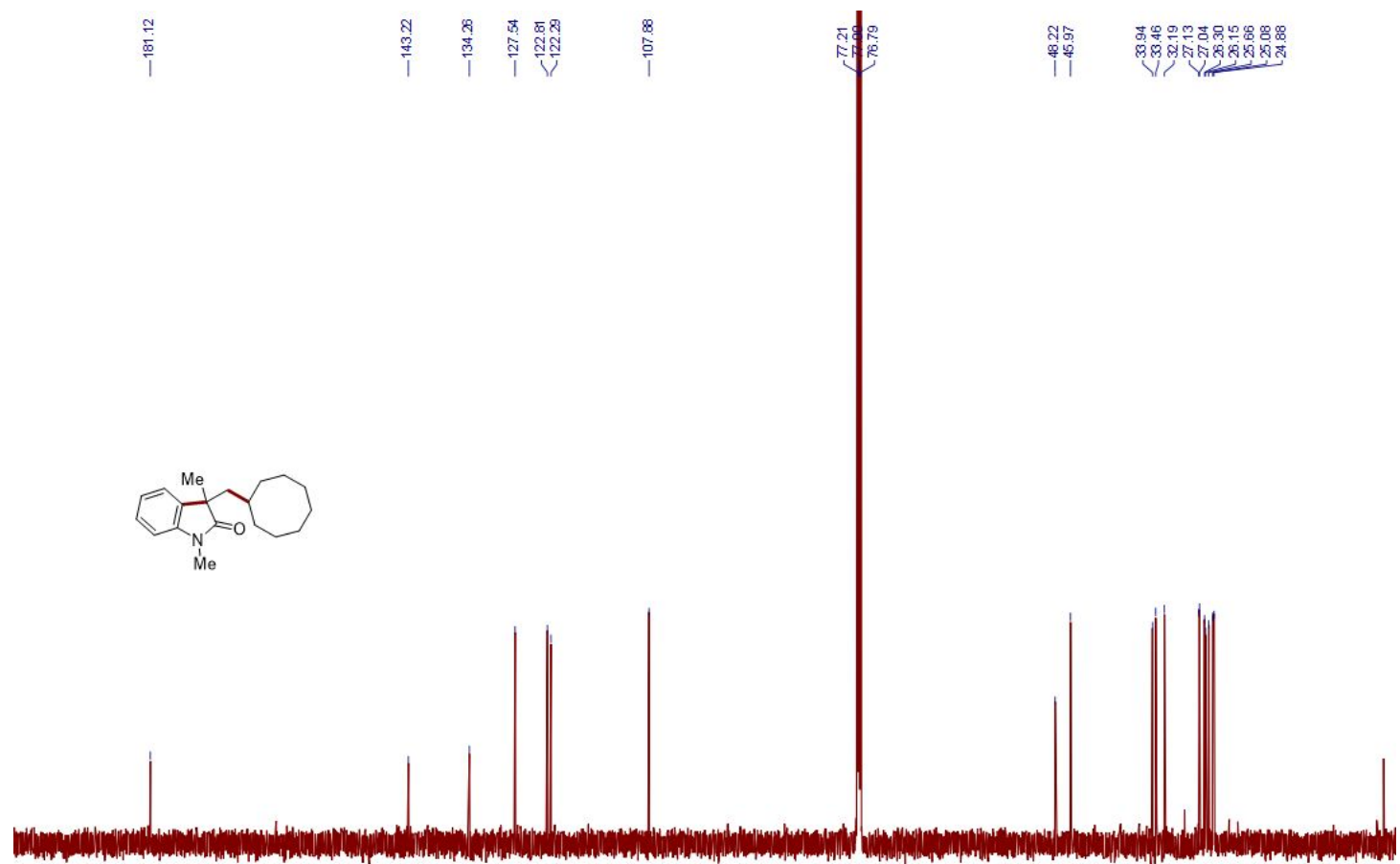

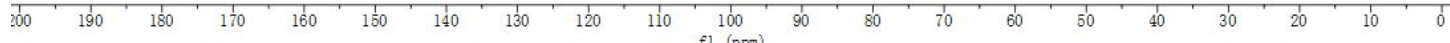


${ }^{1} \mathrm{H}$ NMR of $3 \mathbf{a x}\left(600 \mathrm{MHz}, \mathrm{CDCl}_{3}\right)$

96.
968

\$8ళ

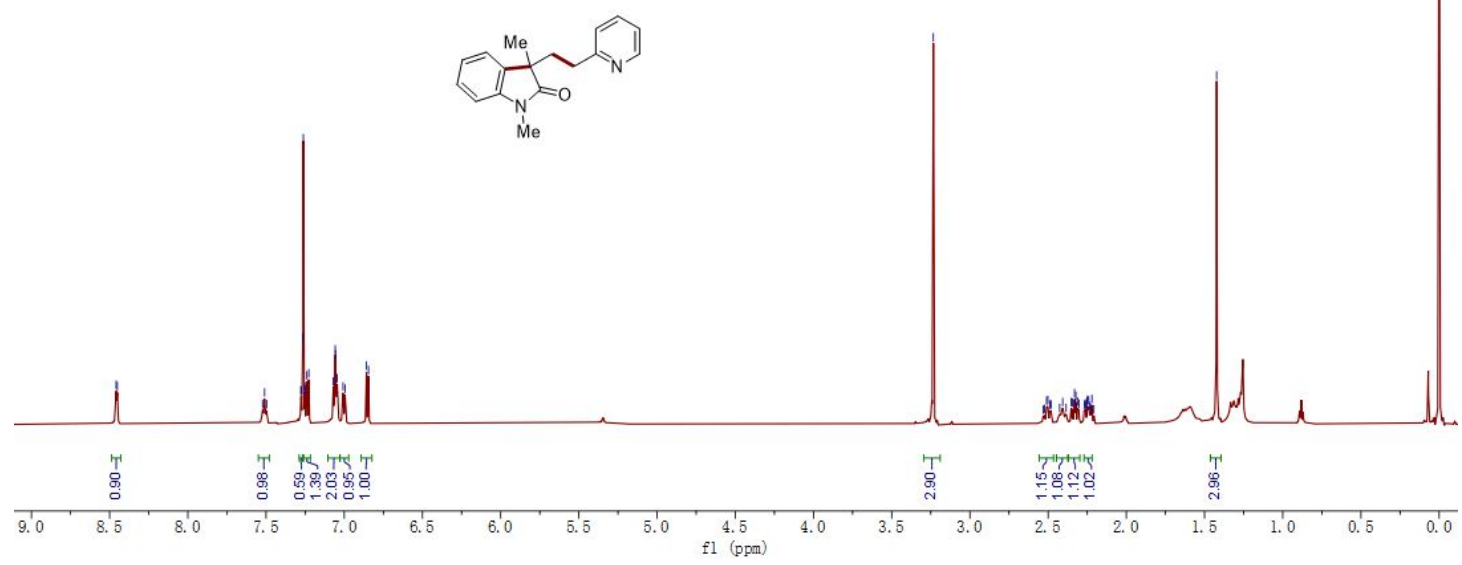

${ }^{13} \mathrm{C}\left\{{ }^{1} \mathrm{H}\right\}$ NMR of $\mathbf{3 a x}\left(150 \mathrm{MHz}, \mathrm{CDCl}_{3}\right)$

$$
\text { 1) }
$$

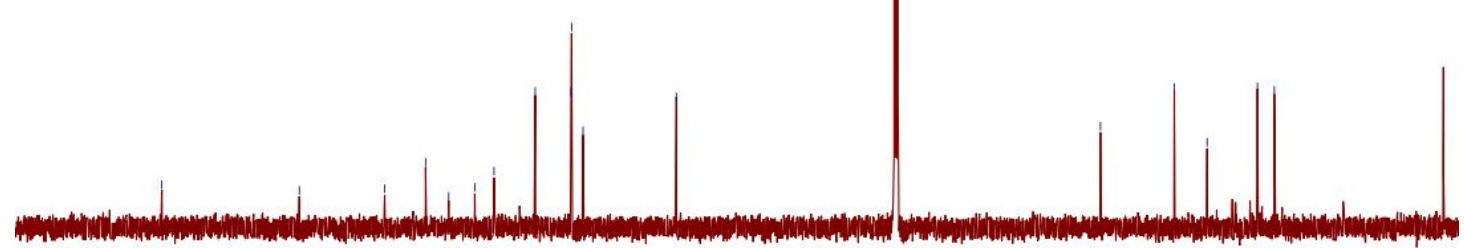

$\begin{array}{llllllllll}100 & 190 & 180 & 170 & 160 & 150 & 140 & 130 & 120\end{array}$ f1 100 
${ }^{1} \mathrm{H}$ NMR of 3 ay $\left(600 \mathrm{MHz}, \mathrm{CDCl}_{3}\right)$

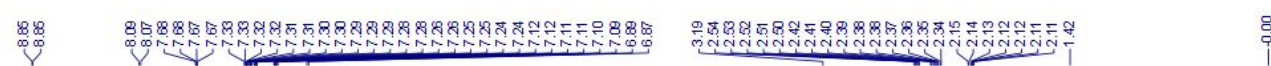

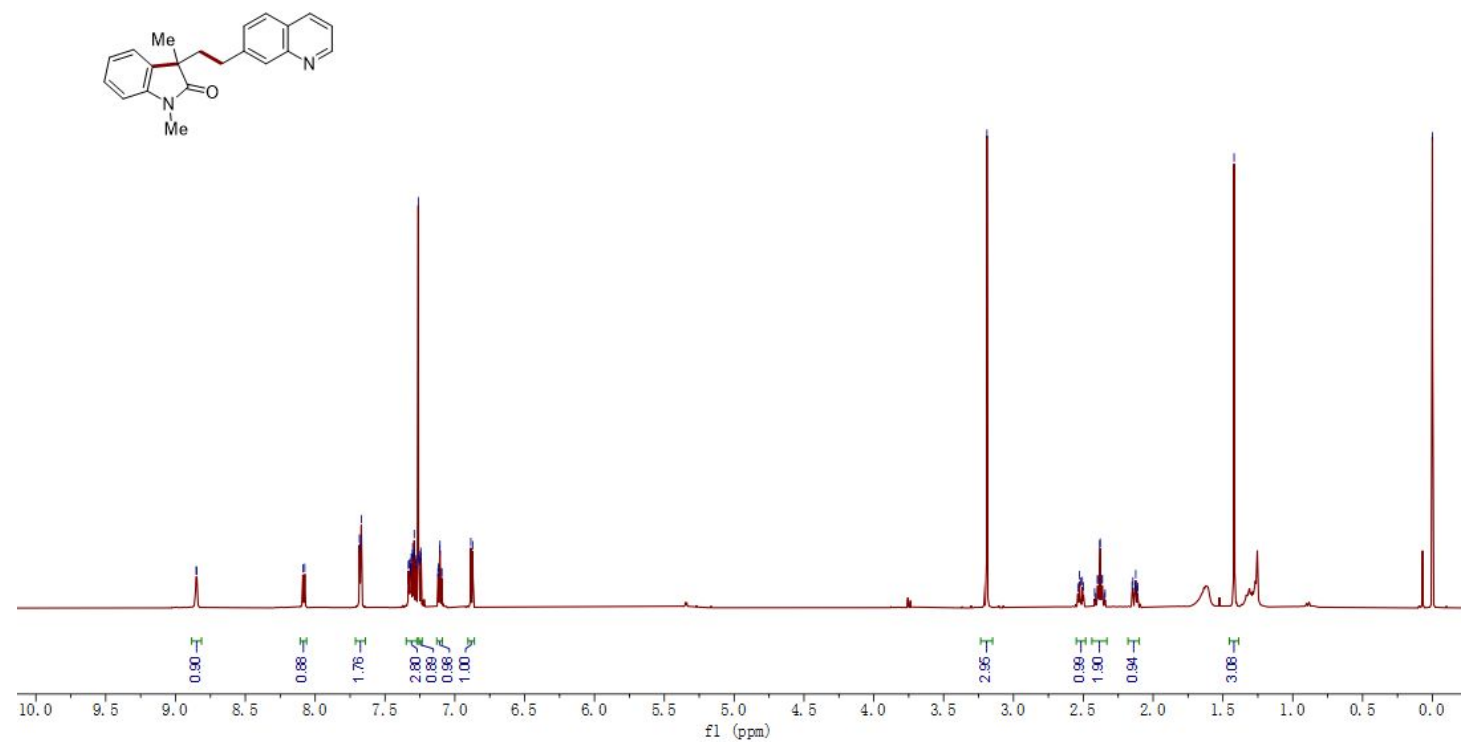

${ }^{13} \mathrm{C}\left\{{ }^{1} \mathrm{H}\right\}$ NMR of 3 ay $\left(150 \mathrm{MHz}, \mathrm{CDCl}_{3}\right)$

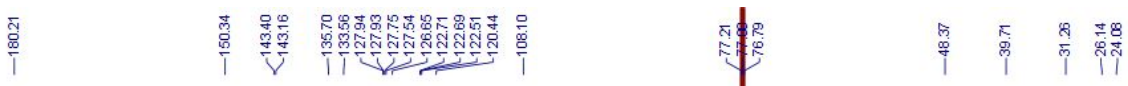<smiles>C[C@]1(CCc2ccc3cccnc3c2)C(=O)N([N+](=O)[O-])c2ccccc21</smiles>

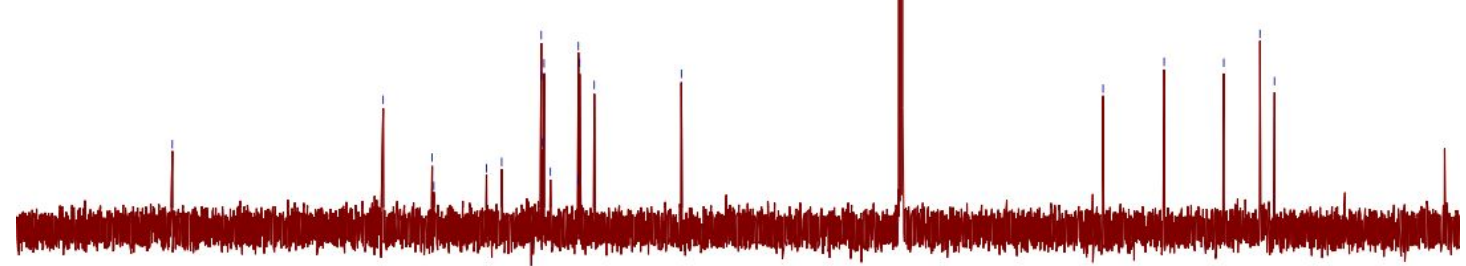

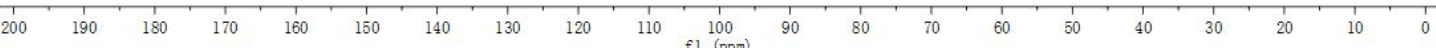


${ }^{1} \mathrm{H}$ NMR of $\mathbf{3 a z}\left(500 \mathrm{MHz}, \mathrm{CDCl}_{3}\right)$

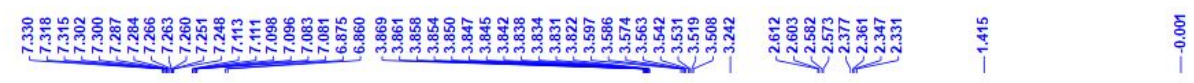
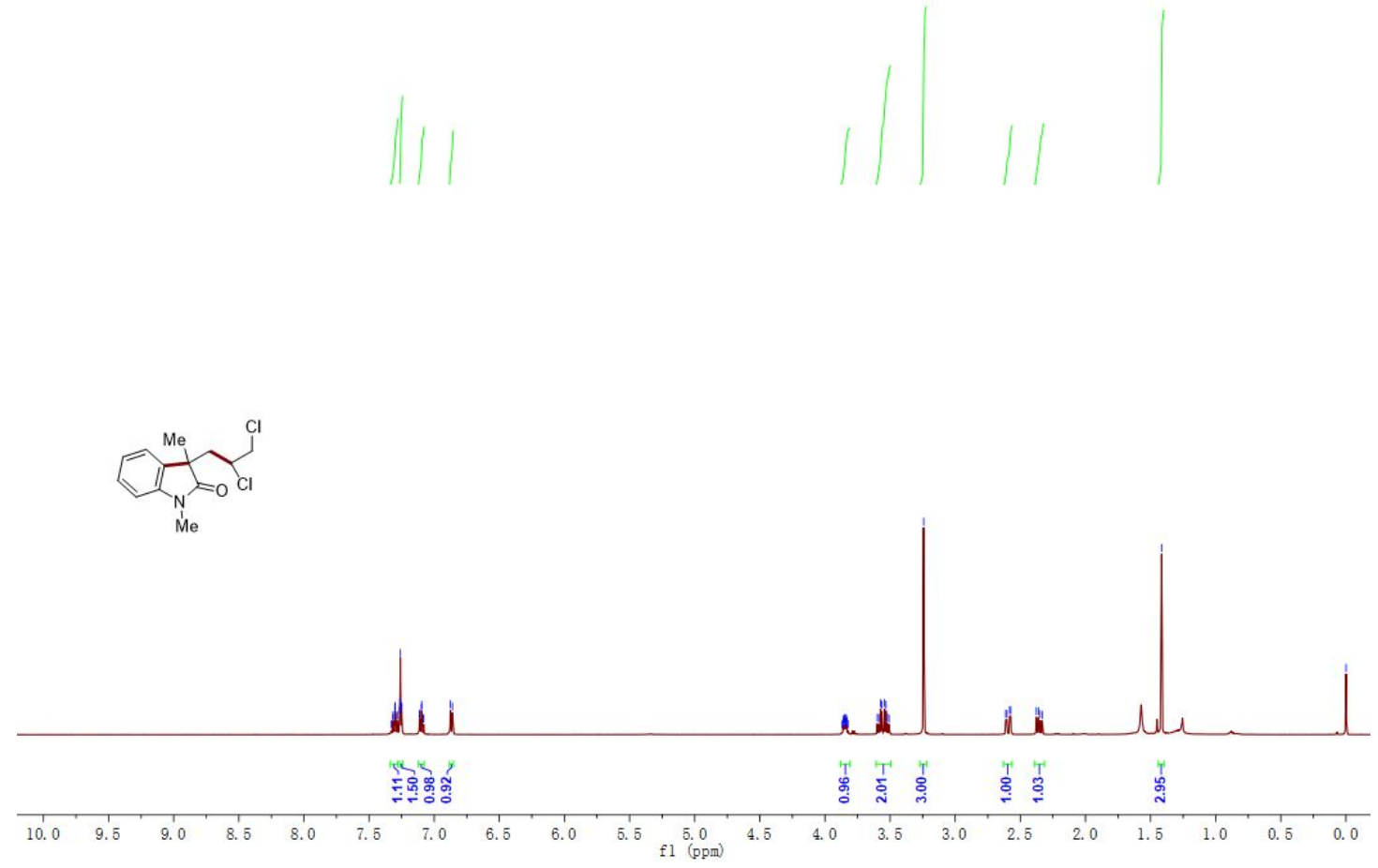

${ }^{13} \mathrm{C}\left\{{ }^{1} \mathrm{H}\right\}$ NMR of $\mathbf{3 a z}\left(125 \mathrm{MHz}, \mathrm{CDCl}_{3}\right)$

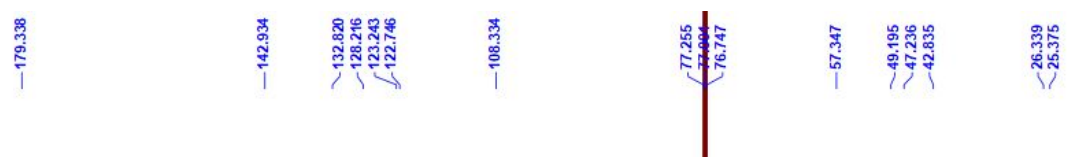

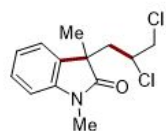

Me

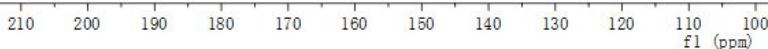


${ }^{1} \mathrm{H} \mathrm{NMR}$ of 3 aza $\left(600 \mathrm{MHz}, \mathrm{CDCl}_{3}\right)$

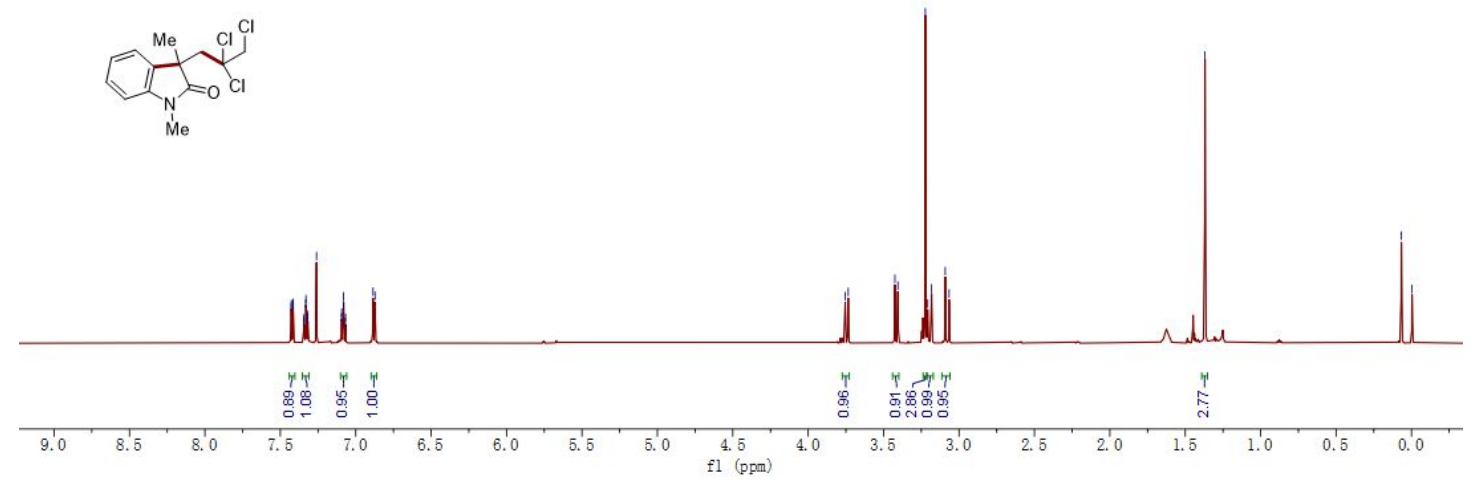

${ }^{13} \mathrm{C}\left\{{ }^{1} \mathrm{H}\right\}$ NMR of 3 aza $\left(150 \mathrm{MHz}, \mathrm{CDCl}_{3}\right)$

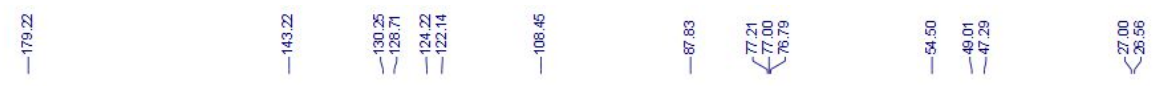
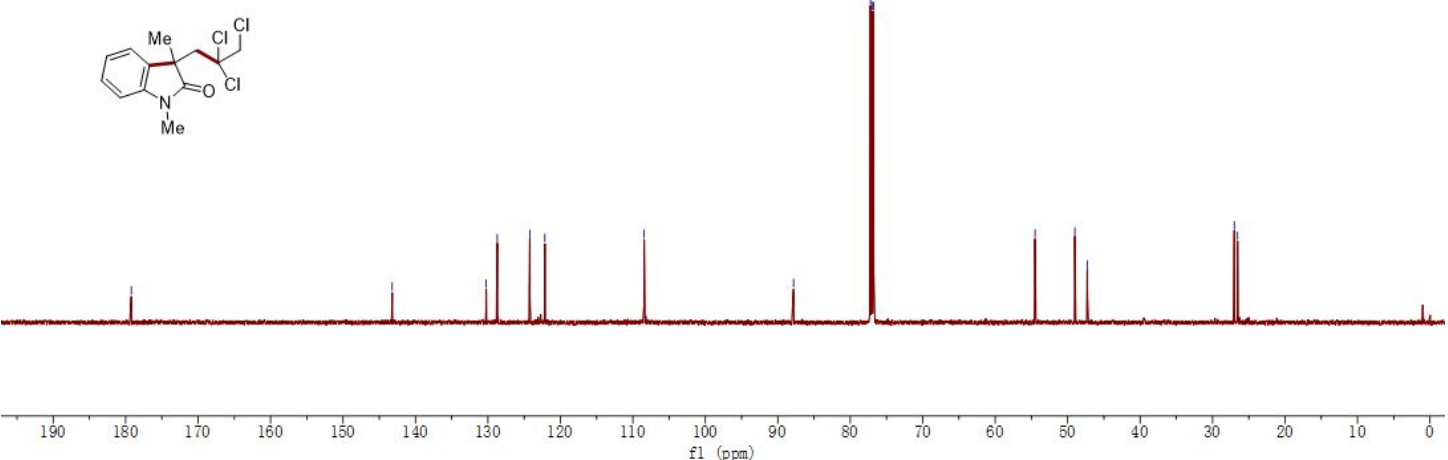
${ }^{1} \mathrm{H}$ NMR of $\mathbf{3 a z b}\left(600 \mathrm{MHz}, \mathrm{CDCl}_{3}\right)$

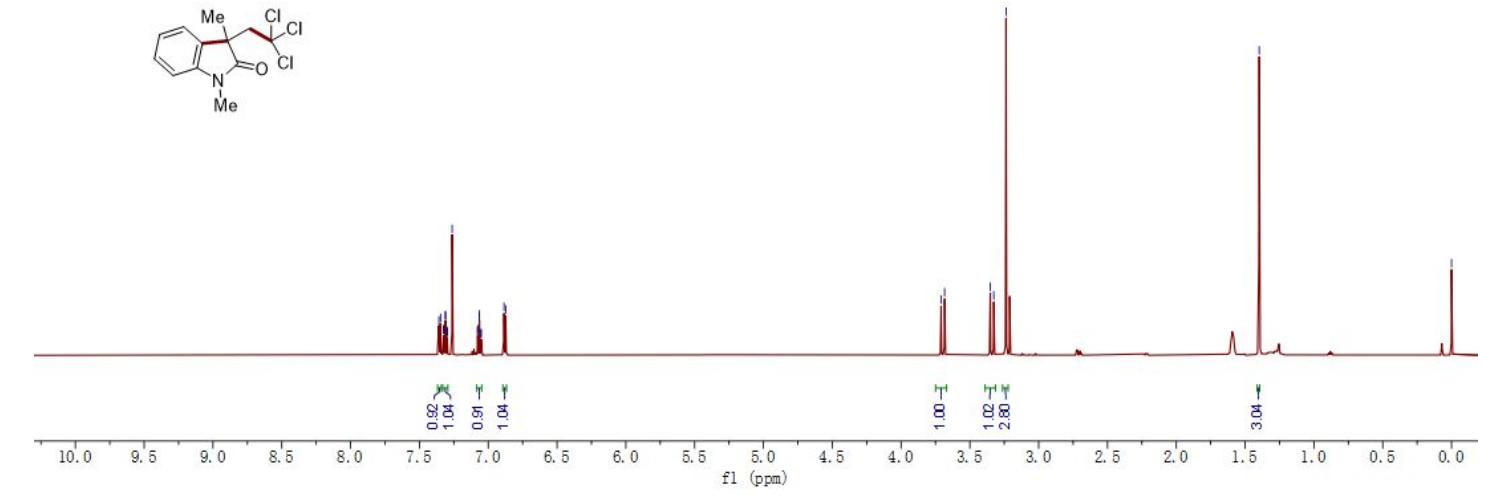

${ }^{13} \mathrm{C}\left\{{ }^{1} \mathrm{H}\right\}$ NMR of $\mathbf{3 a z b}\left(150 \mathrm{MHz}, \mathrm{CDCl}_{3}\right)$

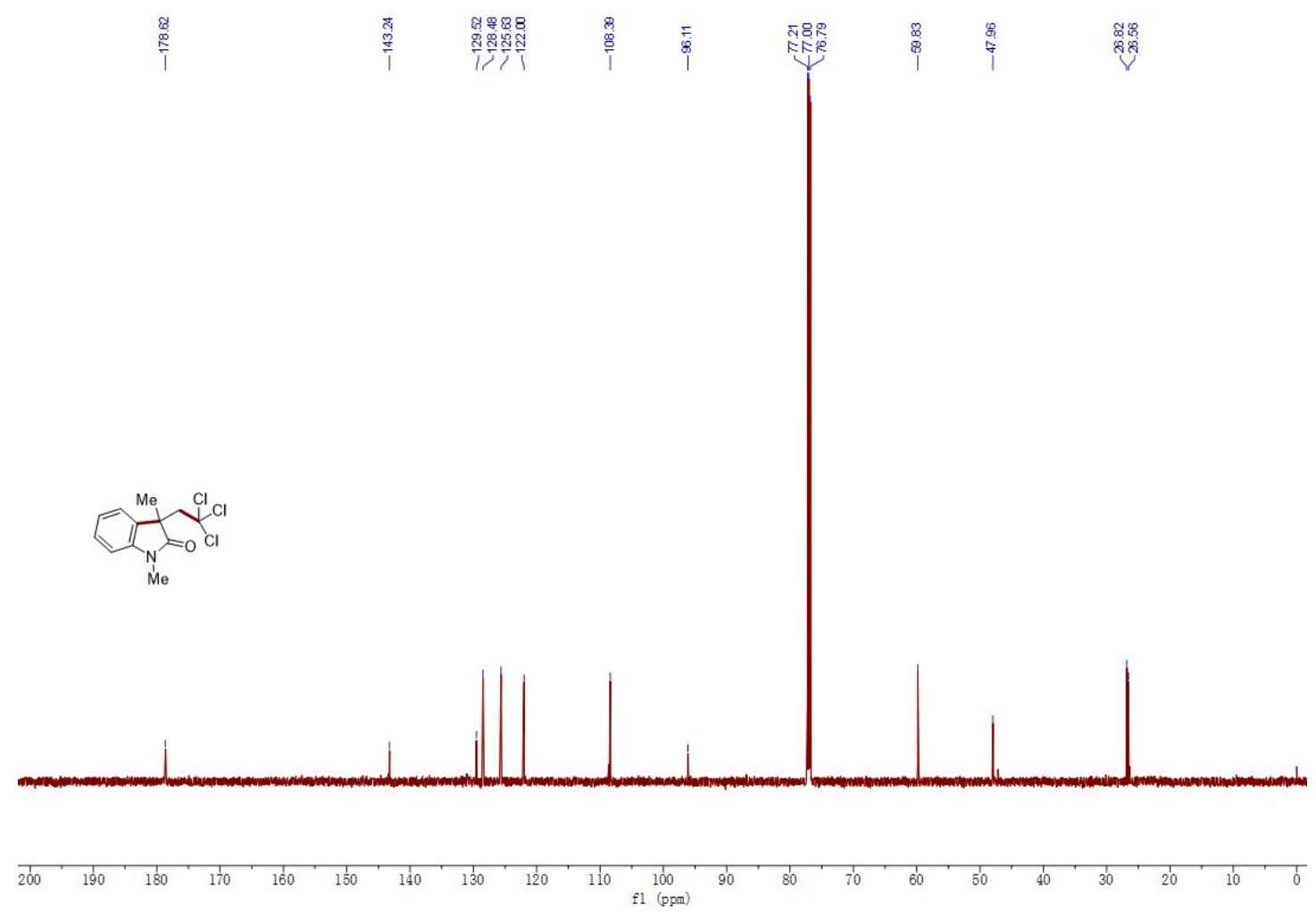


${ }^{1} \mathrm{H} \mathrm{NMR}$ of 3 azc $\left(600 \mathrm{MHz}, \mathrm{CDCl}_{3}\right)$

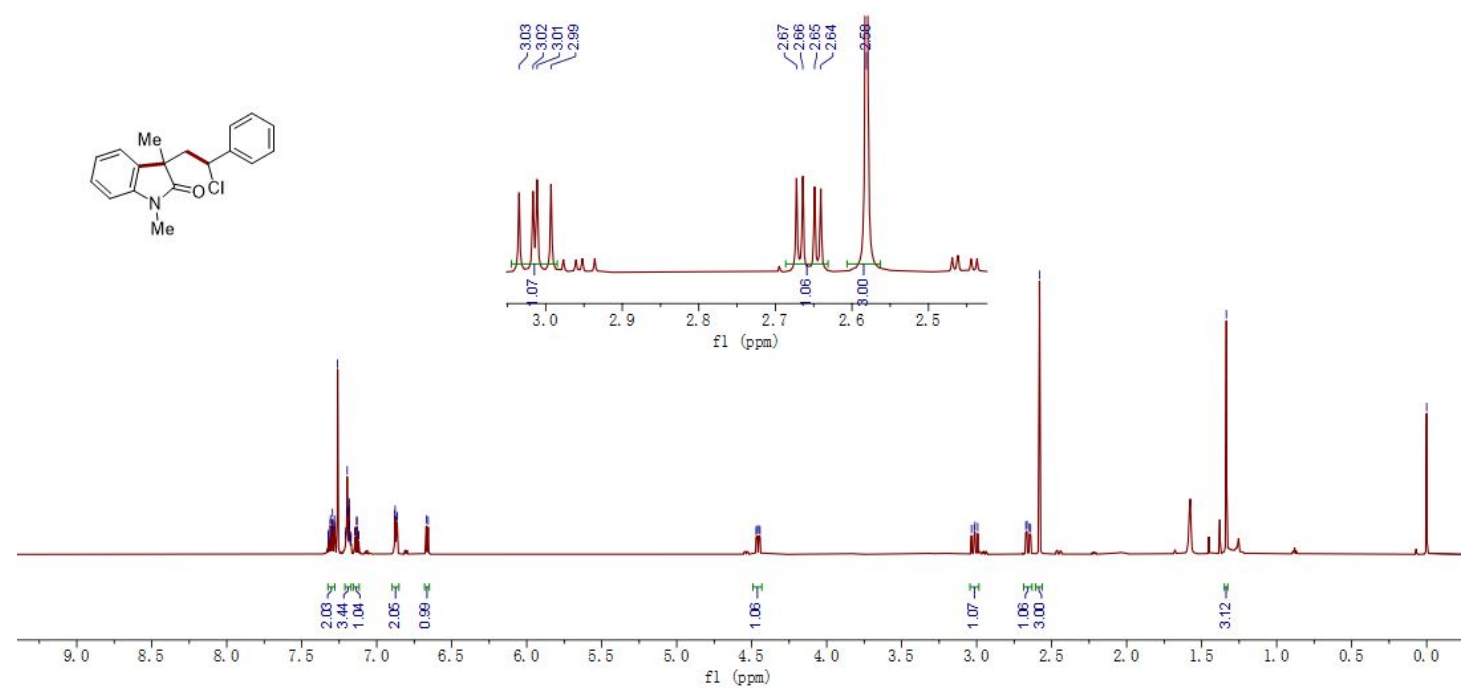

${ }^{13} \mathrm{C}\left\{{ }^{1} \mathrm{H}\right\}$ NMR of $3 \operatorname{azc}\left(150 \mathrm{MHz}, \mathrm{CDCl}_{3}\right)$

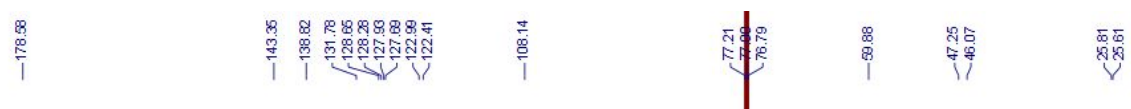

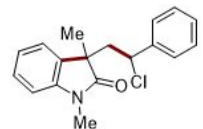

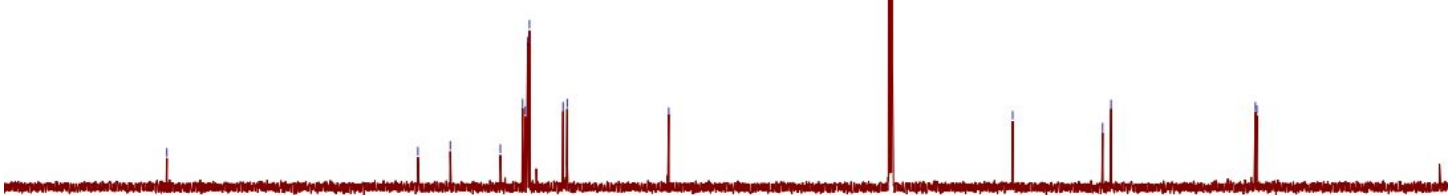

$300 \times 190 \times 180 \times 170 \times 160$

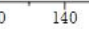

120 $10 \stackrel{100}{10}$ $90 \quad 80$ 
${ }^{1} \mathrm{H} \mathrm{NMR}$ of 3 azd $\left(600 \mathrm{MHz}, \mathrm{CDCl}_{3}\right)$

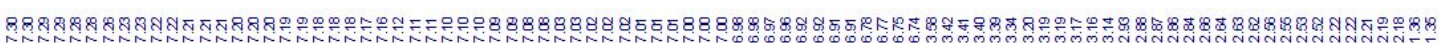

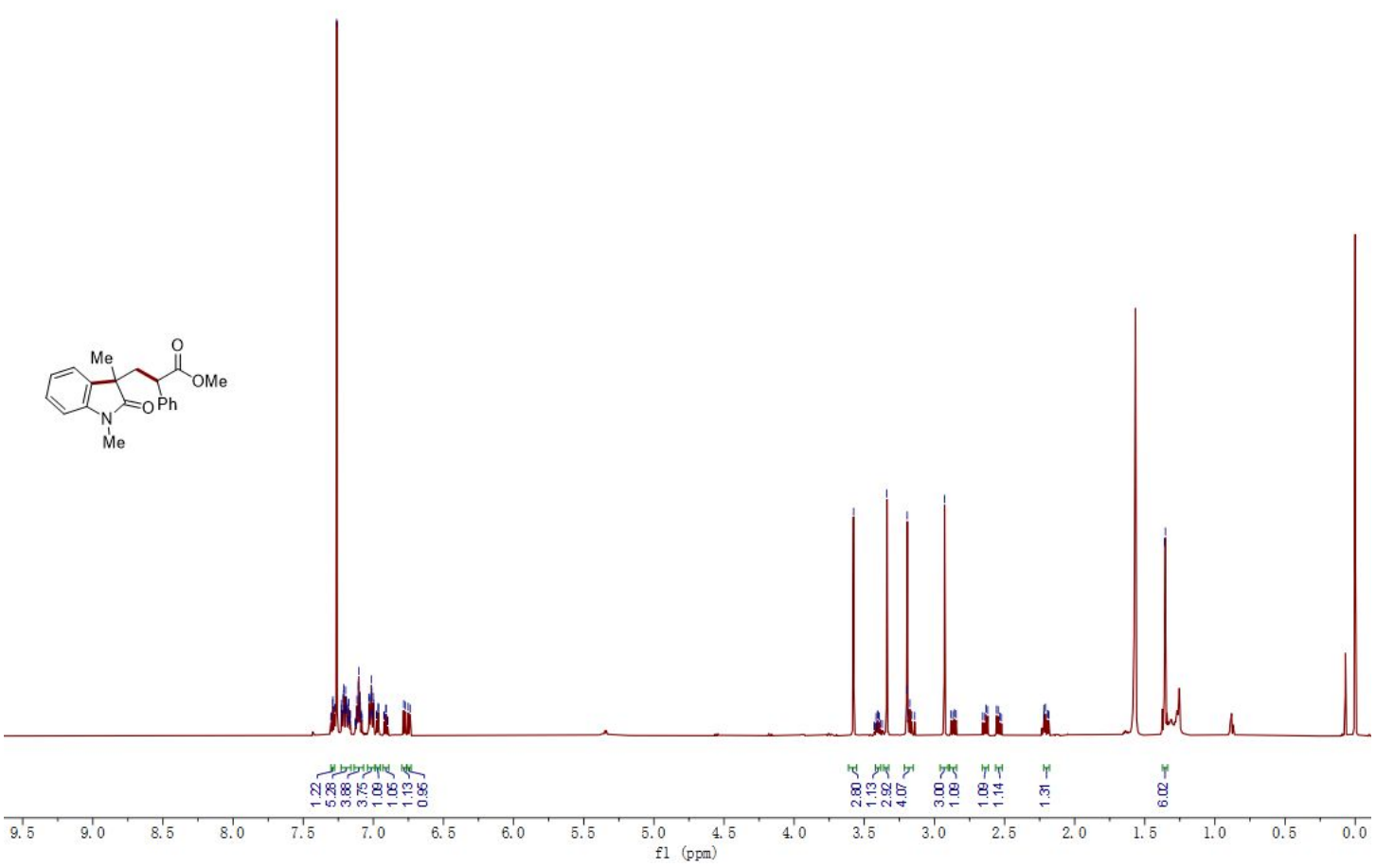

${ }^{13} \mathrm{C}\left\{{ }^{1} \mathrm{H}\right\}$ NMR of 3 azd $\left(150 \mathrm{MHz}, \mathrm{CDCl}_{3}\right)$

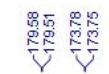
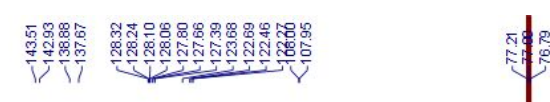

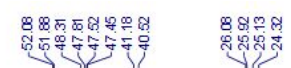

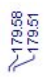

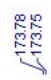

$\sum_{N=O P h}^{M e}$

Me
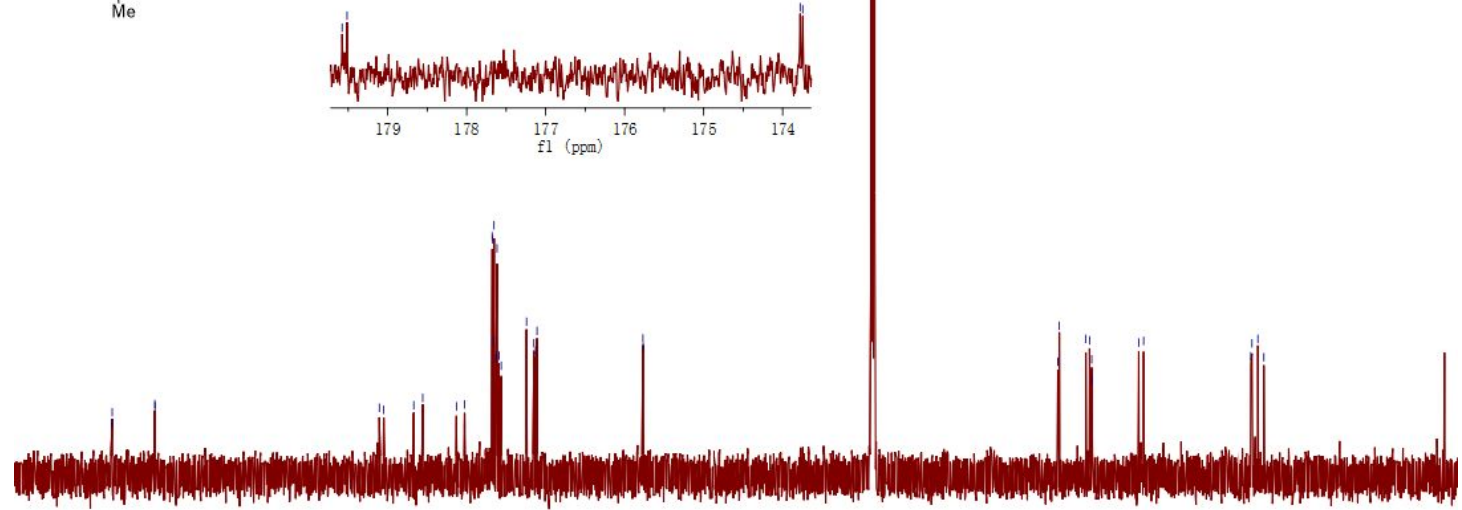

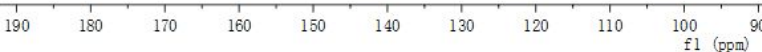


${ }^{1} \mathrm{H}$ NMR of 3aze $\left(600 \mathrm{MHz}, \mathrm{CDCl}_{3}\right)$

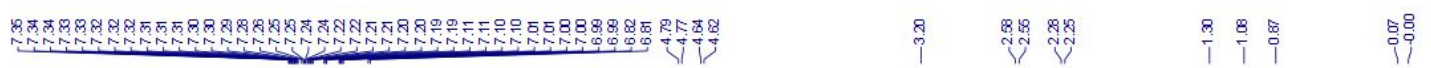

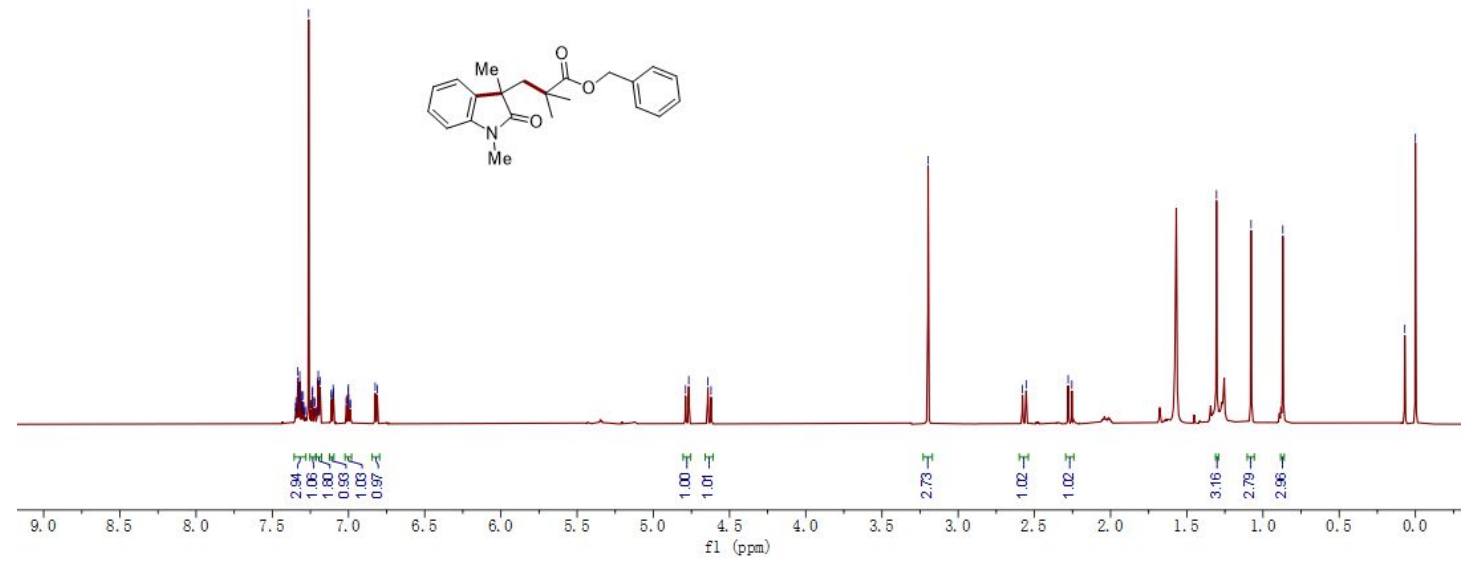

${ }^{13} \mathrm{C}\left\{{ }^{1} \mathrm{H}\right\}$ NMR of 3 aze $\left(150 \mathrm{MHz}, \mathrm{CDCl}_{3}\right)$

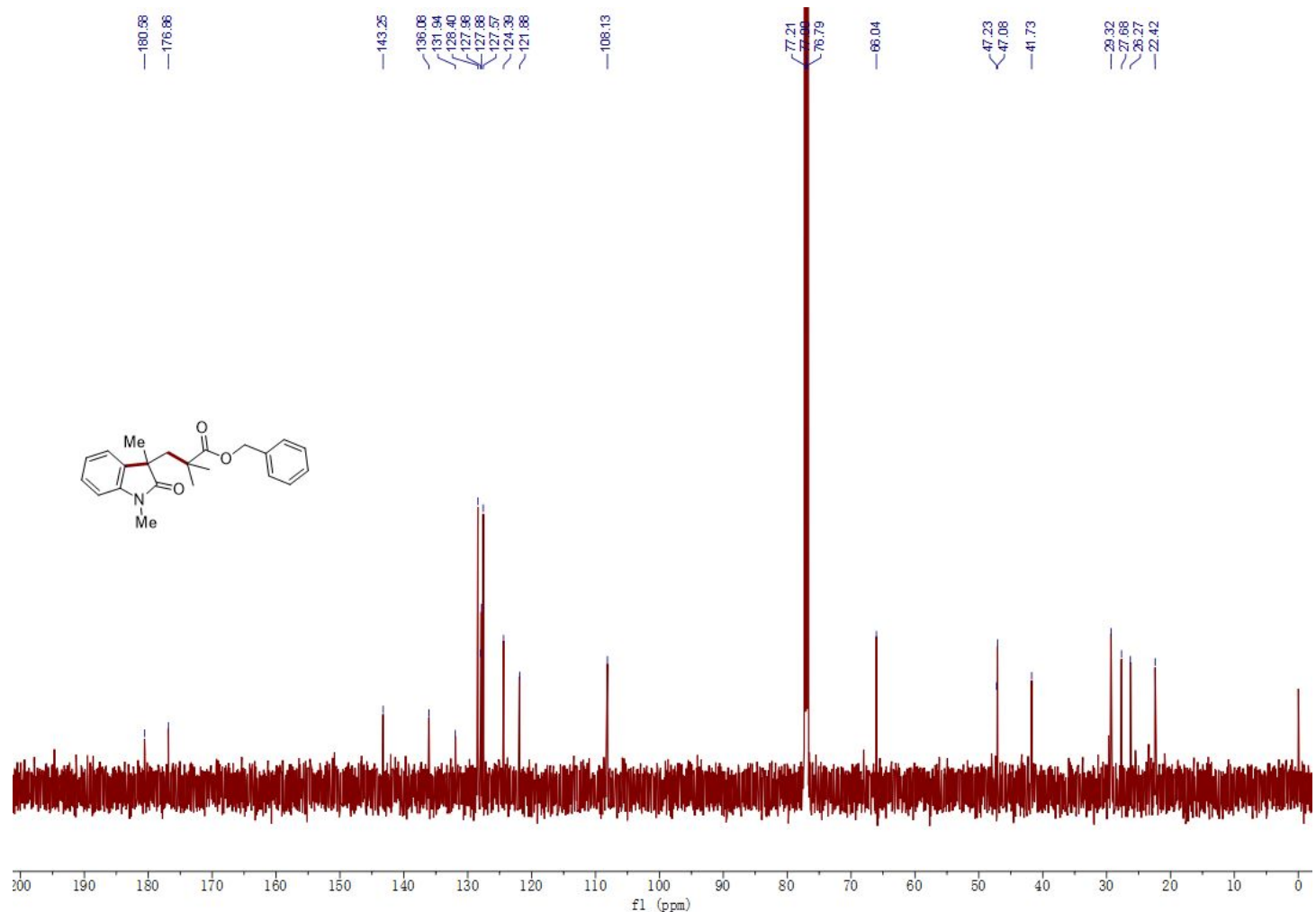


${ }^{1} \mathrm{H}$ NMR of $3 \mathbf{a z f}\left(600 \mathrm{MHz}, \mathrm{CDCl}_{3}\right.$, trace enol tautomer was observed in this spectra)

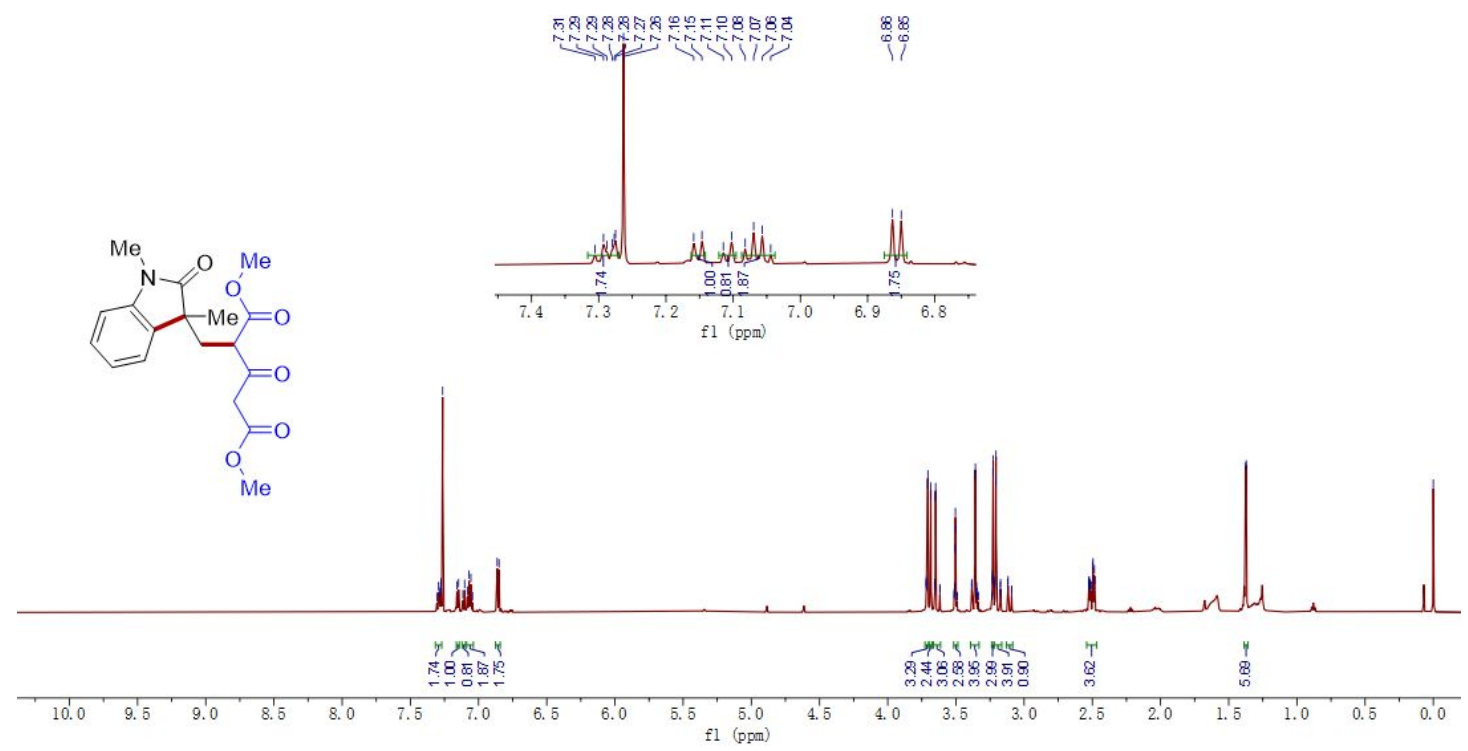

${ }^{13} \mathrm{C}\left\{{ }^{1} \mathrm{H}\right\}$ NMR of $\mathbf{3 a z f}\left(150 \mathrm{MHz}, \mathrm{CDCl}_{3}\right)$
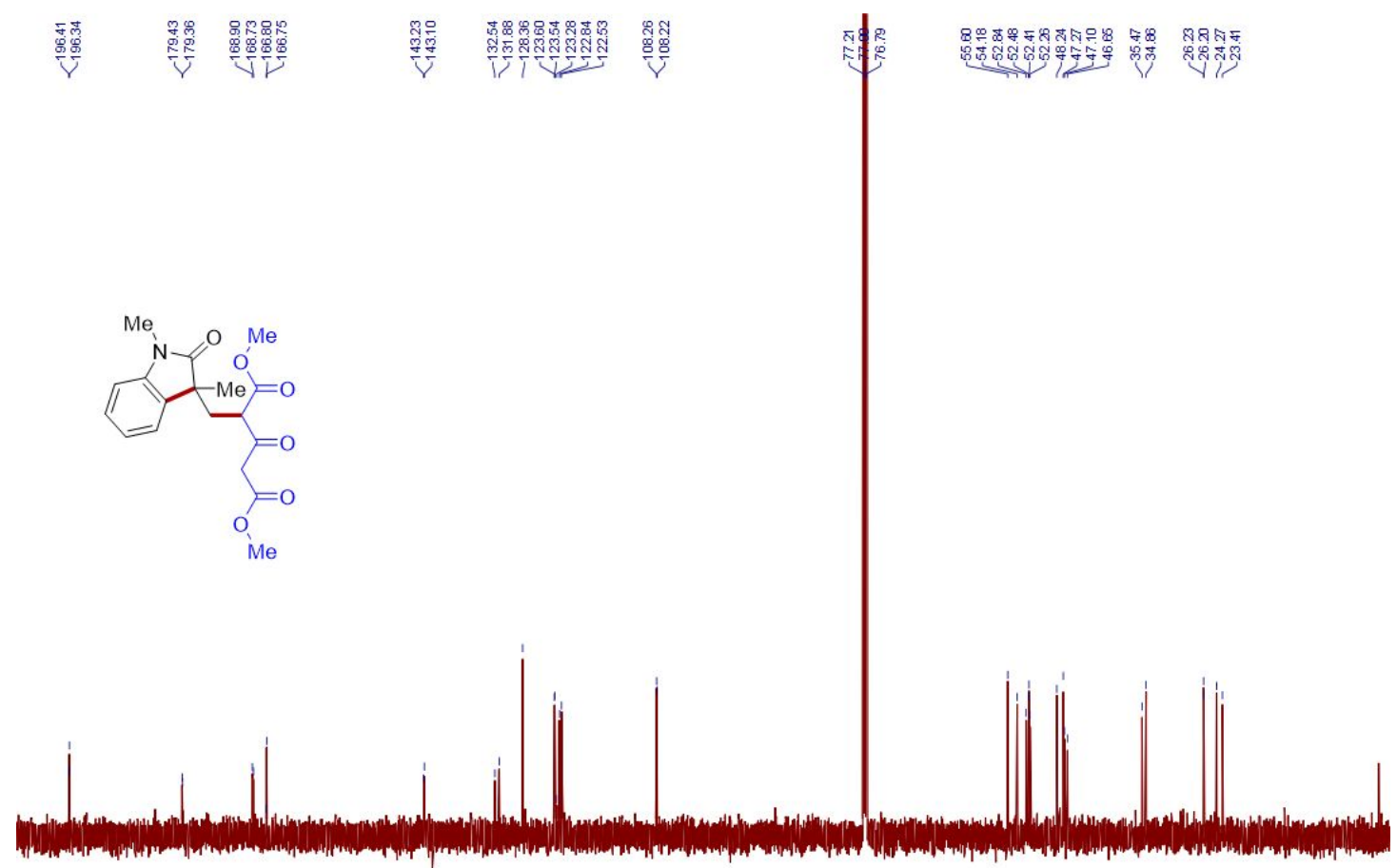

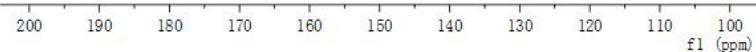


${ }^{1} \mathrm{H}$ NMR of $4\left(400 \mathrm{MHz}, \mathrm{CDCl}_{3}\right)$
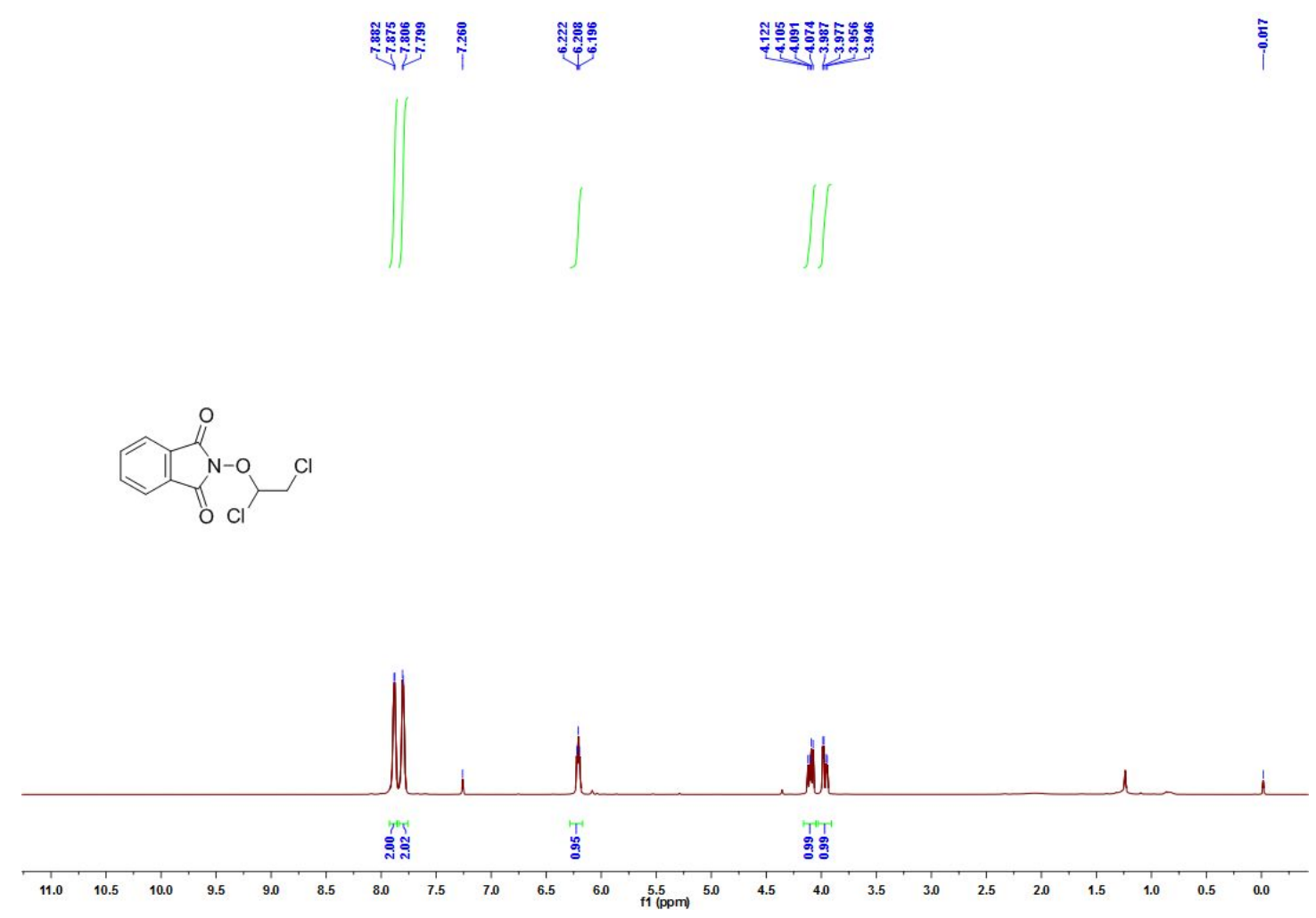

${ }^{13} \mathrm{C}\left\{{ }^{1} \mathrm{H}\right\}$ NMR of $4\left(100 \mathrm{MHz}, \mathrm{CDCl}_{3}\right)$

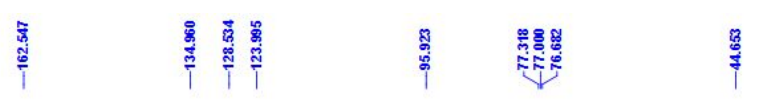

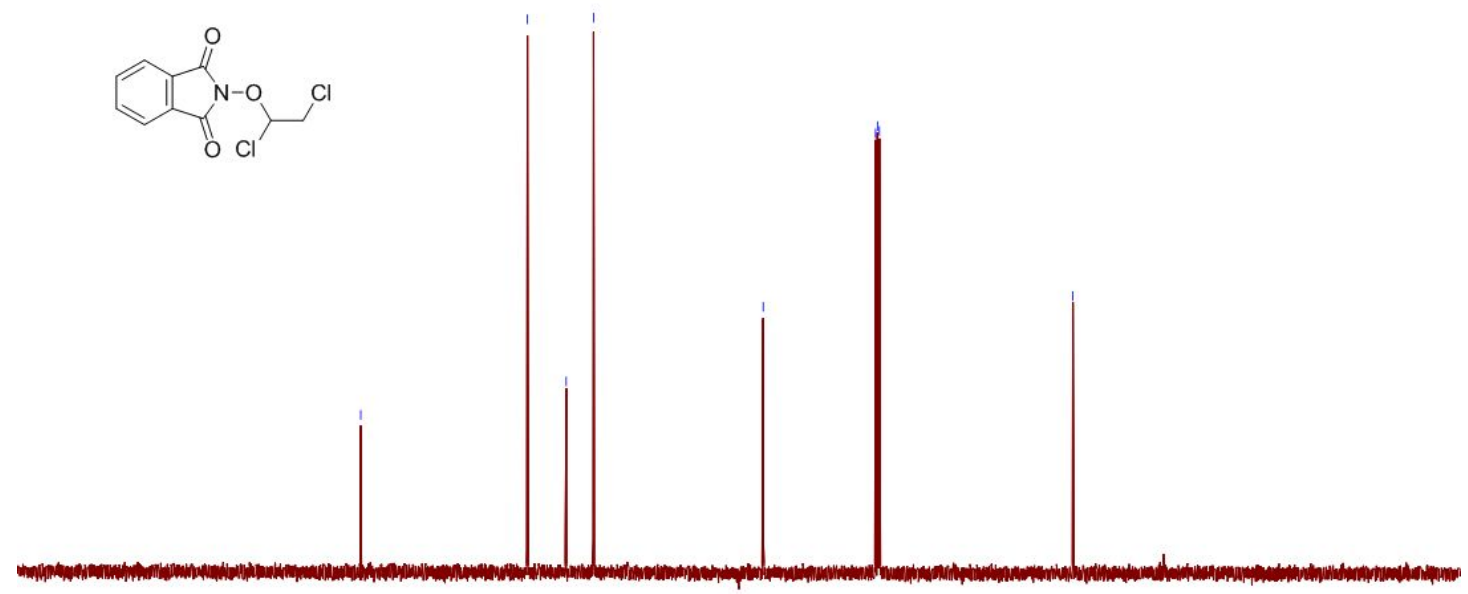

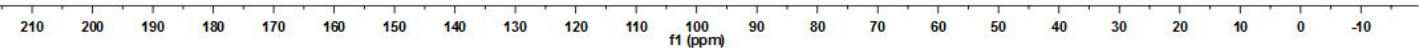


${ }^{1} \mathrm{H} \mathrm{NMR}$ of $5\left(400 \mathrm{MHz}, \mathrm{CDCl}_{3}\right)$
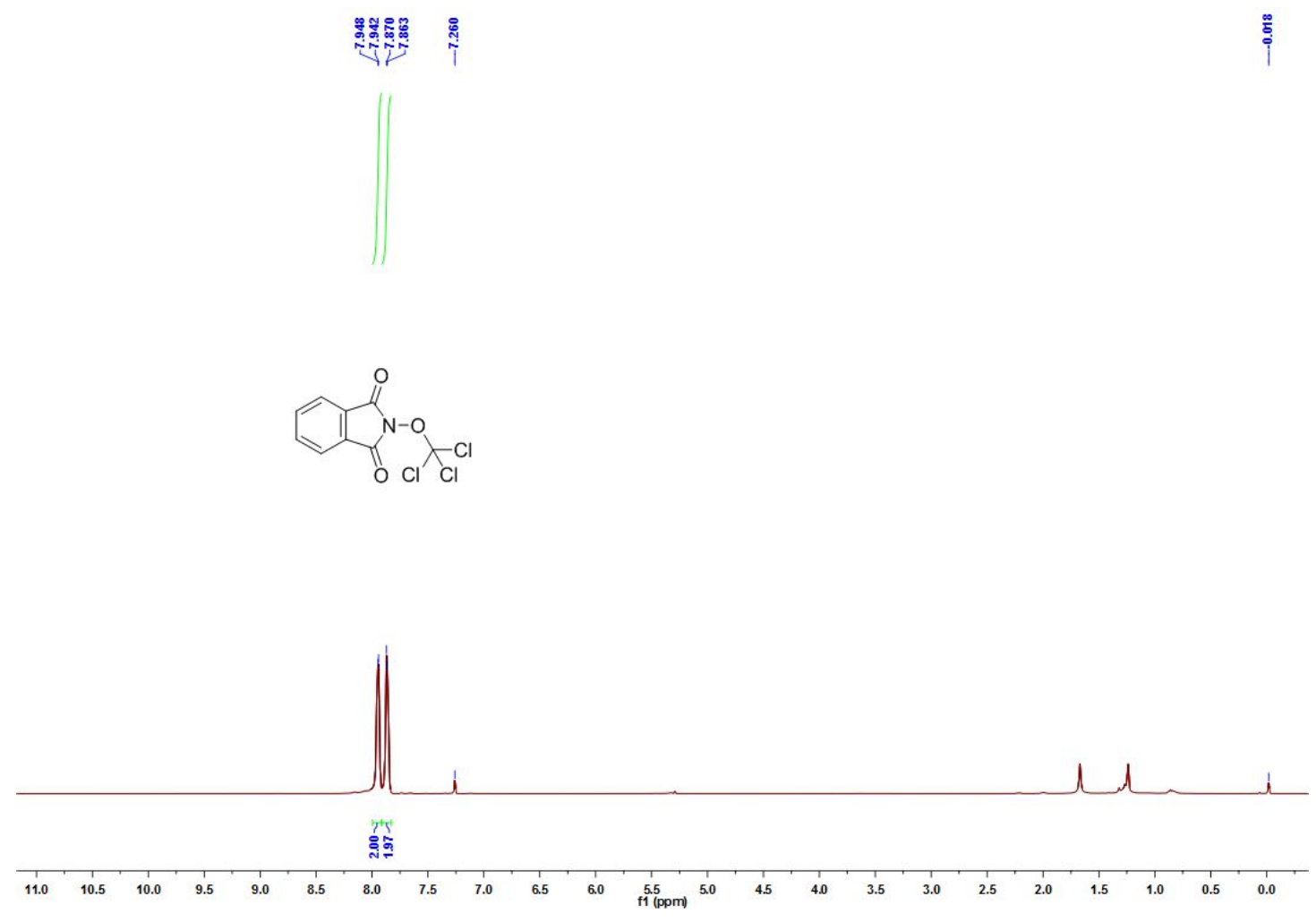

${ }^{13} \mathrm{C}\left\{{ }^{1} \mathrm{H}\right\}$ NMR of $4\left(100 \mathrm{MHz}, \mathrm{CDCl}_{3}\right)$

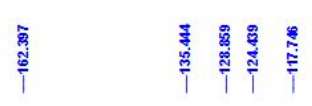

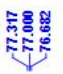
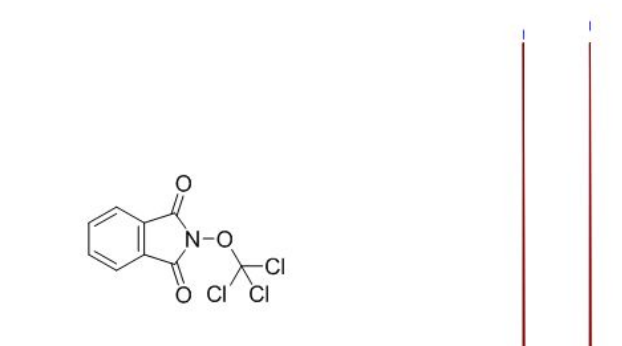

$\begin{array}{lllllll}210 & 200 & 190 & 180 & 170 & 160\end{array}$ 